\title{
The Physical Renormalization of Quantum Field Theories
}

\author{
Michael William Binger
}

SLAC-R-854

Prepared for the Department of Energy

under contract number DE-AC02-76SF00515

Printed in the United States of America. Available from the National Technical Information Service, U.S. Department of Commerce, 5285 Port Royal Road, Springfield, VA 22161. 
This document, and the material and data contained therein, was developed under sponsorship of the United States Government. Neither the United States nor the Department of Energy, nor the Leland Stanford Junior University, nor their employees, nor their respective contractors, subcontractors, or their employees, makes an warranty, express or implied, or assumes any liability of responsibility for accuracy, completeness or usefulness of any information, apparatus, product or process disclosed, or represents that its use will not infringe privately owned rights. Mention of any product, its manufacturer, or suppliers shall not, nor is it intended to, imply approval, disapproval, or fitness of any particular use. A royalty-free, nonexclusive right to use and disseminate same of whatsoever, is expressly reserved to the United States and the University. 


\title{
THE PHYSICAL RENORMALIZATION $\mathrm{OF}$ QUANTUM FIELD THEORIES
}

\author{
A DISSERTATION \\ SUBMITTED TO THE DEPARTMENT OF PHYSICS \\ AND THE COMMITTEE ON GRADUATE STUDIES \\ OF STANFORD UNIVERSITY \\ IN PARTIAL FULFILLMENT OF THE REQUIREMENTS \\ FOR THE DEGREE OF \\ DOCTOR OF PHILOSOPHY
}

Michael William Binger June 2006 
(C) Copyright by Michael William Binger 2006 All Rights Reserved 


\section{Abtract}

The profound revolutions in particle physics likely to emerge from current and future experiments motivates an improved understanding of the precise predictions of the Standard Model and new physics models. Higher order predictions in quantum field theories inevitably requires the renormalization procedure, which makes sensible predictions out of the naively divergent results of perturbation theory. Thus, a robust understanding of renormalization is crucial for identifying and interpreting the possible discovery of new physics. The results of this thesis represent a broad set of investigations into the nature of renormalization. I begin by motivating a more physical approach to renormalization based on gauge-invariant Green's functions. The resulting effective charges are first applied to gauge coupling unification. This approach provides an elegant formalism for understanding all threshold corrections, and the gauge couplings unify in a more physical manner compared to the usual methods. Next, the gauge-invariant three-gluon vertex is studied in detail, revealing an interesting and rich structure. The effective coupling for the three-gluon vertex, $\alpha\left(k_{1}^{2}, k_{2}^{2}, k_{3}^{2}\right)$, depends on three momentum scales and gives rise to an effective scale $Q_{e f f}^{2}\left(k_{1}^{2}, k_{2}^{2}, k_{3}^{2}\right)$ which governs the (sometimes surprising) behavior of the vertex. The effects of nonzero internal masses are important and have a complicated threshold and pseudo-threshold structure. The pinch-technique effective charge is also calculated to two-loops and several applications are discussed. The Higgs boson mass in Split Supersymmetry is calculated to two-loops, including all one-loop threshold effects, leading to a downward shift in the Higgs mass of a few GeV. Finally, I discuss some ideas regarding the overall structure of perturbation theory. This thesis lays the foundation for a comprehensive multi-scale analytic renormalization scheme based on gauge-invariant Green's functions, in which the scale ambiguity problem is reduced since physical kinematic invariants determine the arguments of the couplings. 


\section{Acknowledgement}

First and foremost I wish to thank my advisor, Stan Brodsky, for his exceptional patience, wisdom, and tutelage during my two millennia here at SLAC. In particular, he has the rare ability to always remain good spirited and considerate while working on the mysteries of the universe.

I would also like to thank my Mother and the rest of my family for being so supportive throughout this process.

There are many other friends and colleagues to whom I am indebted for their lessons on physics and life.

The material in Chapter 3 and Chapter 5 has been adopted from the work of Ref.[1] and Ref.[2], respectively, in which Stan Brodsky was a co-author. The material in Chapter 7 has been adopted from the work of Ref.[3]. 


\section{Contents}

Abtract iv

Acknowledgement $\quad$ v

1 Introduction 1

2 Motivations for Physical Renormalization $\quad 6$

2.1 Three Problems with Perturbation theory . . . . . . . . . . . . 6

2.1.1 The Scale/Scheme Ambiguity Problem . . . . . . . . . . 7

2.1 .2 Analyticity . . . . . . . . . . . . . . . 8

2.1.3 Convergence of the series . . . . . . . . . . . . . . . . 9

2.2 Lessons from QED . . . . . . . . . . . . . . . . . . . . . 11

3 Physical Renormalization Schemes and Grand Unification 14

3.1 Introduction . . . . . . . . . . . . . . . . . . . . 14

3.2 Physical Renormalization Schemes and Effective Charges . . . . . 16

3.2.1 A Simple Example . . . . . . . . . . . . . . . . . . . . 17

3.2.2 General Properties of Effective Charges and Physical Renormalization Schemes . . . . . . . . . . . . . . . . 19

3.3 The Canonical Physical Effective Charges of the MSSM . . . . . . . 23

3.4 Unification in Physical Renormalization Schemes . . . . . . . . . . . . 30

3.4.1 The (in) validity of Neglecting Heavy Thresholds . . . . . . . . 30

3.4.2 Heavy Thresholds and Asymptotic Unification . . . . . . . . . 31

3.4.3 Effective Unification Scale . . . . . . . . . . . . . . . . . 40

3.4.4 More General Boundary Conditions . . . . . . . . . . . . . . . 42

3.5 Conclusions . . . . . . . . . . . . . . . . . . . 43

3.6 Appendix A : Pinch Technique Couplings for the MSSM . . . . . . . . 44

3.6.1 QCD Effective Charges . . . . . . . . . . . . . . 45

3.6.2 The Electroweak Sector . . . . . . . . . . . . . . . . . . 47

3.7 Appendix B: Unphysical Schemes and Decoupling . . . . . . . . . 52 
4 Methodology and Selected Results of Precision Gauge Unification $\mathbf{5 7}$

4.1 General Results for the Running Couplings . . . . . . . . . . . . 58

4.1.1 The RGE and thresholds . . . . . . . . . . . . . . 58

4.1.2 Group theory basics ... . . . . . . . . . . . . 60

4.1 .3 One-Loop $\beta_{i}$ coefficients . . . . . . . . . . . . . . . 61

4.1 .4 Two-Loop $\beta_{i j}$ coefficients . . . . . . . . . . . . . . . . 62

4.1.5 Two-Loop Yukawa $Y_{4}^{(i)}(F)$ coefficients . . . . . . . . . 65

4.2 Additional Corrections . . . . . . . . . . . . . . . . 66

4.2.1 Initial value re-calibration of the QED coupling . . . . . . . 66

4.2 .2 Gaugino-Higgsino Mixing . . . . . . . . . . . . . . 67

4.2.3 Non-renormalizable operators (NROs) from Gravity . . . . . . 68

4.3 Methodology and Summary of Corrections . . . . . . . . . . . . 69

4.4 Some examples . . . . . . . . . . . . . . . . . . 71

5 The Form Factors of the Gauge Invariant Three Gluon Vertex $\quad \mathbf{7 5}$

5.1 Introduction: Gauge-Invariant Green's Functions . . . . . . . . . . . 75

5.2 General Structure of the Three-Gluon Vertex . . . . . . . . . . . . . . 80

5.2 .1 Symmetries . . . . . . . . . . . . . . . . . . . 80

5.2 .2 Two Convenient Bases . . . . . . . . . . . . . . . . . . . 82

5.3 Results for the Form Factors . . . . . . . . . . . . . . . . . 88

5.3.1 (Supersymmetric) relations between gluons, quarks, and scalars 90

5.3 .2 The longitudinal form factors . . . . . . . . . . . . . . 95

5.3 .3 The transverse form factors . . . . . . . . . . . . 996

5.3.4 The Form Factors in the Physical Basis . . . . . . . . . . . . . 98

5.4 Three-Gluon Vertex in Perturbation Theory . . . . . . . . . . . . . . 104

5.5 Phenomenological Effects of Internal Masses . . . . . . . . . . . . . 111

5.6 Conclusions and Future Directions . . . . . . . . . . . . . . 118

5.7 Appendix A : Reduction to Scalar Integrals . . . . . . . . . . . . . . 121

5.8 Appendix B : Results for the Triangle Integral . . . . . . . . . . . . . 124

5.9 Appendix C : Special Functions . . . . . . . . . . . . . . . . . . 129

5.10 Appendix D: Supersymmetric Regularization . . . . . . . . . . . . . . 131

5.11 Appendix E : Quark and Squark Mass Corrections . . . . . . . . . . . 132

6 The Pinch-Technique Effective Charge at Two and Three-Loops 136

6.1 Introduction . . . . . . . . . . . . . . . 136

6.2 Notation and Setup . . . . . . . . . . . . . . . . . 137

6.3 Results and Applications . . . . . . . . . . . . . . . . . . . . . . . 139

6.3.1 The effective charge and relation to $\overline{M S} \ldots \ldots . . . . . .139$

$6.3 .2 \mathcal{N}=4$ supersymmetric gauge theory . . . . . . . . . . . 141

6.3 .3 Three-loop $\beta$ function . . . . . . . . . . . . . . . 142 
6.3.4 The longitudinal form factors of the gauge-invariant three-gluon vertex . . . . . . . . . . . . . . . . . . . . 143

6.4 Appendix : Results for Diagrams . . . . . . . . . . . 145

7 The Higgs Boson Mass in Split Supersymmetry at Two-Loops 150

7.1 Introduction . . . . . . . . . . . . . . . . . 150

7.2 Corrections to the Higgs Mass . . . . . . . . . . . . . . 151

7.2.1 The algorithm used to calculate the Higgs mass . . . . . . . 153

7.2 .2 The gaugino and higgsino mass spectrum . . . . . . . . . 154

7.2.3 The Top Quark Yukawa coupling and pole mass . . . . . . . . 156

7.2.4 The 2-loop running of the Higgs Quartic Coupling . . . . . . . 158

7.2.5 The Higgs Self Energy and Tadpole Corrections . . . . . . . . 160

7.3 Results for the Higgs Mass . . . . . . . . . . . . . . . . . . . . 162

8 On the Structure of Perturbation Theory 169

8.1 Predicting Higher Order Terms Using BLM methods . . . . . . . . 169

8.1.1 Banks-Zaks Method . . . . . . . . . . . . . . . . . 172

8.1.2 Results and Limitations . . . . . . . . . . . . . . . . 173

8.2 An Inquiry into the Dominance of Renormalons, BLM Methods, and

Effective Charges Running Inside of Loops . . . . . . . . . . . . . . 174

8.2.1 A Renormalon Inspired Approximation . . . . . . . . . . . 175

8.2 .2 BLM Methods . . . . . . . . . . . . . . . . . . 178

9 Future Directions 182

$\begin{array}{ll}\text { Bibliography } & 187\end{array}$ 


\section{Chapter 1}

\section{Introduction}

Quantum field theories form the foundation for our understanding of three of the four forces of nature. Electromagnetism was the first force to succumb to the charms of a relativistic quantum field theory. Since its inception in the 1940's, Quantum Electro-Dynamics (QED) has been tested and verified to remarkable precision, thus elevating the principle of gauge invariance to the sacrosanct status it holds today. It was not until the 1970's that adequate theories of the strong and weak nuclear forces were developed, also based on the principle of gauge invariance. The strong force is described by Quantum Chromo-Dynamics (QCD), which is based on an unbroken non-abelian symmetry called $S U(3)_{c}$. The weak and electromagnetic forces are mixed together in the so-called Electro-Weak theory [4] based on the gauge group $S U(2)_{L} \times$ $U(1)_{\mathrm{Y}}$, which is partially broken (or hidden) in nature, leaving unbroken only the $U(1)_{\mathrm{EM}}$ symmetry of QED.

Together the $S U(3)_{c} \times S U(2)_{L} \times U(1)_{Y}$ theory, known as the Standard Model (SM) of particles physics, represents one of the great triumphs of twentieth century science. To date, no experimental or theoretical evidence against the SM has been found. All of the particles of the SM have been discovered, with the notable exception of the elusive Higgs boson, which plays the crucial role of breaking the ElectroWeak symmetry and generating the masses of all known particles. There is very good reason to believe that the Higgs boson will be discovered in the near future, most likely at the Large Hadron Collider (LHC) located at CERN, which is due to 
come online in 2007. Moreover, there is also very good reason to believe that the Higgs Boson will not be arriving by itself, but rather with a plethora of new physics associated with the heretofore unknown Higgs sector. The canonical argument for new physics near the electro-weak scale is the so-called hierarchy problem. The technical hierarchy problem derives from the observation that scalar masses (e.g. the Higgs) are generically unstable to radiative corrections from new physics at large energies. This means that if there is some new physics at a scale $M_{N P}$ between the electro-weak scale $\left(M_{E W} \sim 10^{2} \mathrm{GeV}\right)$ and the Planck scale $\left(M_{P l} \sim 10^{19} \mathrm{GeV}\right)$, the minimization of the loop corrected effective potential will lead to a Higgs mass of order $M_{N P}$, unless there is either (a) an extremely unnatural fine-tuning of the parameters of the theory or (b) a "stabilizing" symmetry principle. On the surface, these arguments would seem to suggest against new physics above the electro-weak scale, since it is typically so destabilizing. However, there is likely to be some new physics between $M_{E W}$ and $M_{P l}$, certainly at least some form of quantum gravitation corrections. Thus, particle physicists prefer to take the bull by the horns and try to construct new physics so as to "protect" the Higgs mass from these large unwanted radiative corrections. Once it is protected near the electro-weak scale, it is presumably safe all the way to the Planck scale. These considerations stemming from the hierarchy problem have driven most of the progress in theoretical particle physics in the past twenty-five years, leading to Supersymmetry [5], Technicolor [6], Extra Dimensions [7], and Little Higgs theories [8], to name some of the most prominent ideas. While the discovery of new physics may occur at the LHC, it will take the more precise capabilities of the International Linear Collider (ILC) to distinguish the details of the new physics.

Although past measurements of Charge-Parity $(\mathrm{CP})$ violation are consistent with the SM, it is only the current generation of experiments, BABAR and BELLE, which will rigorously test the SM predictions in a quantitative way. The interplay between experimental and theoretical advances is expected to elucidate the nature of the matter-antimatter asymmetry of the universe and possibly offer another window on new physics.

Given the profound revolution(s) in our understanding of nature that is likely(?!) to emerge in coming years from current and future experiments, it is of the utmost 
importance to have a firm grasp on the precise predictions of the Standard Model, as well as those arising from new physics models. Higher order predictions in quantum field theories inevitably lead to the renormalization procedure, which is needed to make sensible predictions out of the naively divergent results perturbation theory seems to deliver. Thus, the detailed understanding of renormalization is crucial for identifying and interpreting the possible discovery of new physics.

A better understanding of renormalization may also lead to advances in the study of QCD. While the low energy behavior ( $\lesssim 1 \mathrm{GeV}$ ) is likely to require (unknown) theoretical tools beyond perturbation theory, the perturbative regime is plagued by sometimes large uncertainties due to renormalization ambiguities.

This thesis attempts to study various aspects of renormalization, sometimes from a slightly non-standard viewpoint, which we will loosely call "physical renormalization."

In Chapter 2, some motivations for physical renormalization are given. First, some problematic issues with the standard approach to renormalization (in QCD) are outlined, including scale dependence, analyticity, and convergence. The theory of QED is used for guidance in solving these problems.

In Chapter 3, the unification of gauge couplings is studied in physical renormalization schemes, where gauge couplings are defined directly in terms of physical observables. Such effective charges are analytic functions of physical scales, and thus mass thresholds are treated with their correct analytic dependence. In particular, particles will contribute to physical predictions even at energies below their threshold. This is in contrast to unphysical renormalization schemes such as $\overline{M S}$ where mass thresholds are treated as step functions. In this chapter we analyze supersymmetric grand unification in the context of physical renormalization schemes and find a number of qualitative differences and improvements in precision over conventional approaches. The effective charge formalism presented here provides a template for calculating all mass threshold effects for any given grand unified theory. These threshold corrections may be important in making the measured values of the gauge couplings consistent with unification.

In Chapter 4, the methodology for precision gauge coupling unification is synthesized, mostly as a coherent review of known results but with some new formula 
presented. The aim is to be completely general, so as to be able to treat the plethora of unified models on equal footing in regards to the detailed predictions of gaugecoupling unification. The pinch-technique scheme provides an elegant formalism for understanding the various corrections, and the analogous results in the more conventional $\overline{D R}$ scheme can be obtained in a straightforward manner from the pinchtechnique framework. Some results are given to illustrate the current status of gauge coupling unification.

In Chapter 5 , the gauge-invariant three-gluon vertex obtained from the pinch technique is characterized by thirteen nonzero form factors, which are given in complete generality for unbroken gauge theory at one loop. The results are given in $d$ dimensions using both dimensional regularization and dimensional reduction, including the effects of massless gluons and arbitrary representations of massive gauge bosons, fermions, and scalars. We find interesting relations between the functional forms of the contributions from gauge bosons, fermions, and scalars. These relations hold only for the gauge-invariant pinch technique vertex and are d-dimensional incarnations of supersymmetric nonrenormalization theorems which include finite terms. The form factors are shown to simplify for $\mathcal{N}=1,2$, and 4 supersymmetry in various dimensions. In four-dimensional non-supersymmetric theories, eight of the form factors have the same functional form for massless gluons, quarks, and scalars, when written in a physically motivated tensor basis. For QCD, these include the tree-level tensor structure which has prefactor $\beta_{0}=\left(11 N_{c}-2 N_{f}\right) / 3$, another tensor with prefactor $4 N_{c}-N_{f}$, and six tensors with $N_{c}-N_{f}$. In perturbative calculations our results lead naturally to an effective coupling for the three-gluon vertex, $\widetilde{\alpha}\left(k_{1}^{2}, k_{2}^{2}, k_{3}^{2}\right)$, which depends on three momenta and gives rise to an effective scale $Q_{e f f}^{2}\left(k_{1}^{2}, k_{2}^{2}, k_{3}^{2}\right)$ which governs the behavior of the vertex. The effects of nonzero internal masses $M$ are important and have a complicated threshold and pseudo-threshold structure. A three-scale effective number of flavors $N_{F}\left(k_{1}^{2} / M^{2}, k_{2}^{2} / M^{2}, k_{3}^{2} / M^{2}\right)$ is defined. The results of this chapter are an important part of a gauge-invariant dressed skeleton expansion and a related multi-scale analytic renormalization scheme. In this approach the scale ambiguity problem is resolved since physical kinematic invariants determine the arguments of the couplings. 
In Chapter 6, the gauge-invariant gluon self-energy derived from the pinch-technique, or equivalently Background Field Method in Feynman quantum gauge, is calculated explicitly at two-loops, including finite terms. Arbitrary representations of fermions, scalars, and Yukawa couplings are included. Both dimensional regularization (DREG) and the supersymmetric regulator dimensional reduction (DRED) are used. For the $\mathcal{N}=4$ supersymmetric gauge theory, a finite two-loop term remains, in contrast to the one-loop results for the gauge-invariant three-gluon vertex and gluon self-energy. The results of this chapter allow for a more precise numerical determination of the the pinch-technique effective charge, which is derived by re-summing the gauge-invariant gluon self-energy diagrams and is the non-abelian analog of the on-shell charge of QED. Furthermore, the longitudinal form factors of the gauge-invariant three-gluon vertex are determined at two-loops from the Ward identity. The three-loop $\beta$ function coefficient for the pinch-technique charge in QCD is derived.

In Chapter 7, the mass of the Higgs boson in the Split Supersymmetric Standard Model is calculated, including all one-loop threshold effects and the renormalization group evolution of the Higgs quartic coupling through two-loops. The two-loop corrections are very small $(\ll 1 \mathrm{GeV})$, while the one-loop threshold corrections generally push the Higgs mass down several $\mathrm{GeV}$.

In Chapter 8 , some general properties of perturbation theory and renormalization are illustrated through two examples. First, the five-loop terms of order $\alpha_{s}^{4}$ in the Higgs scalar correlator are predicted in the framework of the Brodsky-LepageMackenzie (BLM) scale fixing procedure, and compared with a recent full calculation. Next, the relationship between renormalons, BLM scale fixing, and a hypothetical effective charge running inside of loops is investigated in the context of the two-loop corrections to a heavy quark mass.

In Chapter 9, future directions for research are outlined. 


\section{Chapter 2}

\section{Motivations for Physical Renormalization}

The term physical renormalization is used to distinguish approaches that attempt to relate observables more or less directly to each other, rather than through the use of theoretically defined constructs, such the $\overline{M S}$ coupling of QCD. The on-shell charge of QED developed by Dyson, Gell-Mann, and Low is the canonical example of a physical renormalization scheme. Physical schemes enjoy superior analytic properties and have no renormalization scale ambiguity, since the scales are naturally related to physical kinematic invariants. These points will be discussed in detail throughout the next few sections, and recur throughout this thesis.

\subsection{Three Problems with Perturbation theory}

In perturbative $\mathrm{QCD}$ ( $\mathrm{pQCD}$ ), the preferred scheme among most practitioners is modified minimal-subtraction $(\overline{M S})$. $\overline{M S}$ has some very positive calculational advantages, due to the fact that it is truly the "minimal" subtraction scheme: only the divergence and associated constants are absorbed into the bare coupling in order to define the renormalized coupling.

Despite these attributes, the conventional formulation of $\mathrm{pQCD}$ as an expansion in the $\overline{M S}$ coupling has three main problems : 
- Renormalization Scale/Scheme Ambiguity

- Analyticity through thresholds and over timelike and spacelike momenta

- Renormalons which lead to a divergent perturbation series

As we will see in the next three subsections, these problems are related to each other.

\subsubsection{The Scale/Scheme Ambiguity Problem}

In perturbative calculations, one of the dominant sources of theoretical uncertainty is the ambiguity in choice of renormalization scale and scheme. For a generic single-scale observable calculated to order $N$ in perturbation theory,

$$
R(Q)=\sum_{n=0}^{N} R_{n}(Q, \mu) \alpha_{s}^{n}(\mu)
$$

the scale and scheme of the highest order term is considered ambiguous since their variation leads to a correction which is formally of higher order $(N+1)$ :

$$
\begin{aligned}
\alpha_{s}\left(\mu^{\prime}\right) & =\alpha_{s}(\mu)-\frac{\alpha_{s}^{2}(\mu)}{2 \pi} \beta_{0} \log \frac{\mu^{\prime}}{\mu} \\
\alpha_{s}^{\prime}(\mu) & =\alpha_{s}(\mu)+C \alpha_{s}^{2}(\mu) .
\end{aligned}
$$

Note that a scheme shift can also be viewed as a scale shift at leading order,

$$
\begin{aligned}
\alpha_{s}^{\prime}(\mu) & =\alpha_{s}(\bar{\mu})+\mathcal{O}\left(\alpha_{s}^{3}\right) \\
\bar{\mu} & =\mu \exp \left(\frac{-2 \pi C}{\beta_{0}}\right) .
\end{aligned}
$$

Several proposals have been advanced in order to deal with the scale and scheme uncertainty.

- The Principle of Minimal Sensitivity (PMS) [9] demands that the perturbative expansion be as insensitive as possible to the variation of scheme and scale parameters. In practice usually only scale variation is considered. 
- The Fastest Apparent Convergence (FAC) [10, 11] method demands that the scale should be fixed so as to make the perturbative expansion appear the most convergent. This is done by choosing the scale such that the highest order term computed vanishes.

- In the Brodsky-Lepage-Mackenzie (BLM) [12] method, the scales are chosen at each order to eliminate the terms which depend on the number of flavors $N_{f}$. The BLM method is inspired by an analogy with QED, as will be explained shortly.

\subsubsection{Analyticity}

The $\overline{M S}$ charge is not analytic through mass thresholds and does not distinguish between time-like and space-like virtualities. In $\overline{M S}$, the particles contributing to the renormalization of the coupling constant are treated as massless, so that they are either fully "turned on" or fully "turned off." When the scale of the problem is lower than the mass of some species of quark, that quark must be decoupled by hand. This artificial decoupling leads to a set of schemes and couplings, defined in each desert region, which must be mapped together. To any order in perturbation theory one can in principle derive matching conditions which relate the couplings. To leading order one simply has $\alpha_{s}^{(f+1)}\left(M_{Q}\right)=\alpha_{s}^{(f)}\left(M_{Q}\right)$, and the coupling has discontinuous derivative at $M_{Q}$. At higher orders the coupling itself becomes discontinuous. In section $3.7 \mathrm{a}$ more detailed account of the decoupling of massive particles will be given.

In addition, the lack of a distinction between time-like and space-like momenta means, for example, that the appropriate imaginary parts of higher order terms are missing. While these terms are formally of higher order, they can be taken into account consistently, as is done in the physical effective charge scheme furnished by the pinch-technique which is considered in Chapters 3,5 , and 6 .

Finally, the issue of analyticity near the origin of the $q^{2}$ plane brings us to the question of the convergence of perturbation theory. 


\subsubsection{Convergence of the series}

It is commonly believed that QCD perturbation series is divergent due to the effects of so-called renormalons, as illustrated in Fig.(2.1). The renormalon analysis of QCD assumes (a) the existence of a gauge-invariant combination of radiative corrections which has the structure of gluon self-energy insertions, and (b) these corrections are the dominant ones at any order of perturbation theory. Provided these assumptions are true, the bubble chain insertions will lead to a factorial growth in the series coefficients $R_{n} \propto n !$. This can be heuristically understood as the integration over extremely small momenta where a running coupling inside loops becomes very large. Thus, it is usually stated that pQCD has an asymptotic series, for which there is some optimal number of terms that should be computed, and after which the series begins to diverge.

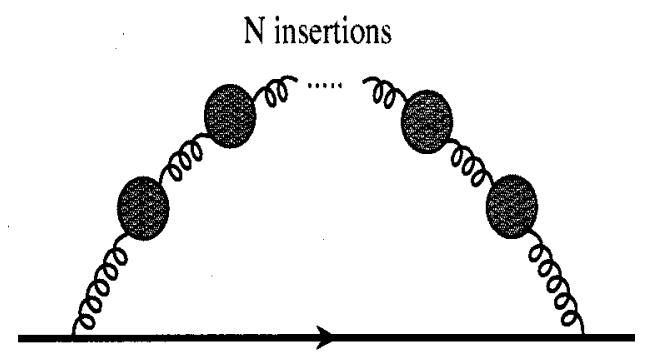

Figure 2.1: Renormalon chain in a quark propagator.

Consider the loop integral

$$
\int d^{4} l \alpha_{s}\left(l^{2}\right) f\left(l^{\mu}, p_{i}^{\mu}\right)
$$

where $f$ contains the propagators and numerator while $\alpha_{s}\left(l^{2}\right)$ comes from re-summing the gluon self-energy insertions. Using a scheme that does not distinguish between timelike and spacelike momenta, such as $\overline{M S}$, leads to

$$
\alpha_{s}\left(l^{2}\right)=\frac{4 \pi}{\beta_{0} \log \left|l^{2} / \Lambda_{\overline{M S}}^{2}\right|}
$$

which is singular for both spacelike and timelike momenta of $l^{2} \approx \pm \Lambda \frac{2}{M S}$. In contrast, 
using an effective charge which distinguishes spacelike and timelike momenta leads to

$$
\widetilde{\alpha}\left(l^{2}\right)=\frac{4 \pi}{\beta_{0}\left(\log \left|l^{2} / \widetilde{\Lambda}^{2}\right|-i \pi \theta\left(l^{2}\right)\right)},
$$

so that the infrared renormalon is regulated by the imaginary part for timelike momenta $\left(|\widetilde{\alpha}|<\frac{4}{\beta_{0}} \approx 4 / 9\right)$, although it persists for spacelike momenta.

However, there is by now compelling evidence that QCD effective charges have an infrared fixed-point $[13,14,15]$, defined by $\widetilde{\alpha}\left(Q^{2} \rightarrow 0\right) \rightarrow$ constant. Although formally beyond fixed order perturbation theory, using some phenomenological fixedpoint behavior for the effective charge will remove even the spacelike IR renormalon divergence.

If indeed QCD is the complete theory of the strong interactions, then there should be a meaningfully defined perturbative expansion that is convergent, not asymptotic. This may be achieved if either

1. the running coupling (defined as some physical effective charge) has a finite IR fixed point $[13,14,15]$, or

2. the assumptions underlying the renormalon analysis, namely the existence and dominance of renormalon contributions, proves false.

Thus far, we have not specified how to construct running charges inside of loops in QCD. Indeed, no satisfactory method has been yet been developed to accomplish this task $^{1}$. In the past, there has been some speculation that the pinch-technique justifies the renormalon analysis by offering a graphical understanding for the construction of gauge-invariant gluon self-energy. In section 8.2, some arguments will be given against this proposal, i.e. that a pinch-technique charge cannot be unambiguous constructed inside of loops. Section 8.2 is a preliminary effort towards understanding the relationship between the existence and dominance of renormalons, BLM scale fixing, and a hypothetical dressed skeleton expansion where charges run inside of loops.

\footnotetext{
${ }^{1}$ See the work of Neubert $[16,17]$ for another approach, which starts with the assumption of running charges inside of loops.
} 


\subsection{Lessons from QED}

In Quantum Electro-Dynamics (QED) the above three problems are essentially eliminated. For the scale ambiguity and analyticity problems, this happy situation arises largely due to the existence of a dressed-skeleton-expansion, wherein the virtuality of each photon line uniquely determines the scale of the coupling at the vertex. QED has a very natural and physically motivated scheme, sometimes called the on-shell scheme, with an analytic coupling known as the on-shell or Gell Mann-Low--Dyson effective charge $e\left(q^{2}\right)$, which approaches the fine structure constant $\alpha=\frac{e^{2}(0)}{4 \pi} \approx(137.036)^{-1}$ in the limit of zero photon virtuality. Of course, the improved convergence properties of QED are related to the IR fixed point behavior, which trivially follows because the theory is Abelian. We will now consider these features of QED in more detail.

Consider the simple example of $e^{-} e^{-}$scattering in QED, which at leading order consists of two graphs: $t$ channel and $u$ channel. The $t$-channel graph is shown in Fig.(2.2). Re-summing the photon vacuum polarization diagrams naturally leads to $e(t)$ at both vertices, where $t$ is the Mandelstam variable, and there is no scale ambiguity in this approach. Nevertheless, if one insists on choosing a different scale $\mu^{2} \neq t$ for this diagram, then there will be an infinite series of corrections proportional to $\left(e^{2}\left(\mu^{2}\right) \log \mu^{2} / t\right)^{n}$ at each order $n$, which, when re-summed, lead once again to $e^{2}(t)$. Similarly, the $u$-channel graph (not shown) is proportional to $e^{2}(u)$, so we can write the renormalization improved amplitude schematically as

$$
\mathcal{M}=\mathcal{M}_{t} e^{2}(t)+\mathcal{M}_{u} e^{2}(u)
$$

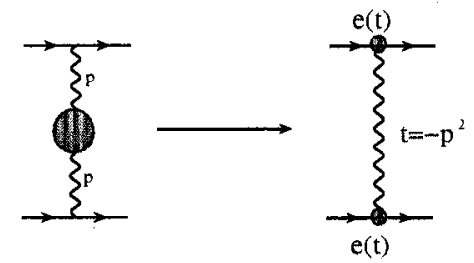

Figure 2.2: QED has no scale ambiguity. 
If instead $\overline{M S}$ is used, then the scale must apparently be guessed, since there is no natural diagrammatic understanding of the scale, unlike in the Gell-Man-Low-Dyson scheme, where the physical kinematic invariants $t$ and $u$ are the scales. Typically in $\overline{M S}$ a single scale is chosen for all vertices, so that

$$
\mathcal{M}_{\overline{M S}}=\left(\mathcal{M}_{t}+\mathcal{M}_{u}\right) \bar{e}^{2}\left(\mu^{2}\right) .
$$

In general, one can solve for the correct $\overline{M S}$ scale $\mu$ by squaring the amplitudes and and equating with the on-shell result given above. The resulting scale is unlikely to be correctly guessed.

Now consider the possibility of choosing different $\overline{M S}$ scales for each graph. Perhaps physical intuition will lead one to choose the $\overline{M S}$ scales $\mu^{2}=t$ and $\mu^{2}=u$ for the two graphs. However, this is incorrect, as can be seen by the relation $e^{2}\left(Q^{2}\right)=e_{\overline{M S}}^{2}\left(Q^{2} e^{-5 / 3}\right)+\mathcal{O}\left(e^{6}\right)$, which implies that the correct scales in the $\overline{M S}$ scheme are $e^{-5 / 3} t$ and $e^{-5 / 3} u$, which are also unlikely to be guessed.

The errors due to the wrong scale choice are formally of higher order. However, using a running charge is supposed to account for a certain subclass of dominant higher order corrections. It is not clear this is happening when the scale is ambiguous, since there is only one choice of scale that re-sums these terms correctly. Note that it is not the infrared fixed point of QED that allows one to unambiguously fix the scale. Rather, it is the relative simplicity in the construction of the effective charge from a gauge-invariant subset of diagrams.

There are also useful lessons from QED in regards to analyticity. The physical QED charge is in general complex at timelike momenta, with precisely the correct imaginary parts dictated by unitarity and analyticity. Furthermore, the coupling has smooth behavior through mass thresholds. These attributes can be seen in the process $e^{+} e^{-} \rightarrow \mu^{+} \mu^{-}$, shown in Fig.(2.3), which has a complex analytic charge defined by re-summing the vacuum polarization graphs, with the imaginary part, $\operatorname{Im} e^{2}(-s) \propto \operatorname{Im} \Pi_{\gamma \gamma}(s)$, determined by the physical cuts of the vacuum polarization graph.

Finally, the renormalon problem does arise formally in pure QED. However, in this 


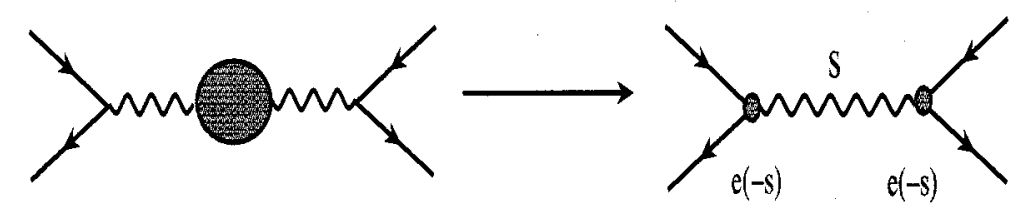

Figure 2.3: The QED coupling is a complex analytic function of physical scales.

case it is the so-called UV renormalons which come from loop momentum integration near the Landau singularity $\left(\gg M_{P l}\right)$. Since QED is merged into the electro-weak theory well below the Landau pole, there is no problem. Here QED offers little guidance for the QCD renormalon problems, since it is highly unlikely that QCD merges with another theory at hadronic energy scales.

With the exception of the Landau singularity at unphysically large energies, the QED charge $\alpha\left(q^{2}\right)$ is analytic over the $q^{2}$-plane. $\overline{M S}$ does not share this feature.

To summarize, QED offers several important lessons on the construction of a physical renormalization scheme:

- The effective couplings are complex analytic functions with the correct threshold structure expected by unitarity.

- Multiple "renormalization" scales appear in a physical process.

- The scales are totally unambiguous since they are related to physical kinematic invariants.

The generalization of these attributes to non-abelian theories will appear in later chapters. A useful check is that QCD must match on to an Abelian theory in the limit of $N_{c} \rightarrow 0$ [18]. 


\section{Chapter 3}

\section{Physical Renormalization Schemes and Grand Unification}

\subsection{Introduction}

Precision measurements of the gauge couplings and their possible unification provides one of the few windows to the Planck scale. It is thus important to have a firm grasp of the theoretical ambiguities involved. This chapter attempts to address some of these ambiguities.

In a physical renormalization scheme, gauge couplings are defined directly in terms of physical observables. Such effective charges are analytic functions of physical scales, and thus the thresholds associated with heavy particles are treated with their correct analytic dependence. This is in contrast to unphysical renormalization schemes such as the $\overline{M S}$ scheme where mass thresholds are treated as step functions. In this chapter we will analyze supersymmetric grand unification in the context of physical renormalization schemes with the goal of systematizing the effects of light and heavy mass thresholds and improving the precision of tests of unification compared with conventional approaches.

In section 3.2 , we motivate physical renormalization schemes with a simple example and then present the notation and results used throughout the chapter. In 
section 3.3, we discuss the canonical self-energy-like effective charges for the Minimal Supersymmetric Standard Model (MSSM). These effective couplings run smoothly over spacelike momenta, have non-analytic behavior only at the expected physical thresholds for timelike momenta, and more directly measure the strengths of the forces than the charges of unphysical schemes. The extraction of effective charges from low energy data is considered. We identify an important modification of the electromagnetic coupling $\alpha_{\mathrm{QED}}\left(M_{Z}\right)$ due to the proper inclusion of virtual $W^{ \pm}$loops, thus resulting in a $4 \sigma$ change in its numerical value. Similar modifications are found for the weak mixing angle. As seen in section 3.4, these effective charges provide a more natural and physical framework for examining gauge coupling unification. In section 3.4.1, we demonstrate the invalidity of neglecting heavy threshold corrections in analyzing grand unified models. The more rigorous treatment of light thresholds in physical schemes gives rise to new corrections, but these are numerically small for most sparticle spectra. The treatment of heavy thresholds with various unification boundary conditions is discussed in section 3.4.2. In the simplest scenario, we find that the gauge couplings should unify at asymptotically large energies and the only heavy threshold corrections are logarithms of heavy mass ratios, which can be obtained in unphysical schemes. An effective unification scale, defined in section 3.4.3 as the scale where quantum gravity corrections produce non-negligible splittings between the gauge couplings, is found to be roughly $10^{17}-10^{18} \mathrm{GeV}$, depending on the specific GUT model used. Section 3.4.4 considers more general unification boundary conditions with finite unification scale. The resulting heavy threshold corrections are given in Eq.(3.40). This result combined with the results of section 3.4.2 may be used to determine the experimental consistency of any given GUT model. Appendix A discusses the details of constructing the effective charges. Appendix B looks more carefully at the problem of decoupling heavy particles and the errors induced by unphysical schemes.

There have been several previous works on threshold effects in grand unification. In the first such study [19], which appeared just after the discovery of the grand unification, D.A. Ross used form factors to define $\beta$ functions which are valid over all energy scales, including near mass thresholds. The coupling constants run smoothly 
over all momenta, and nontrivial threshold corrections are found for grand unification. Despite this early significant work, most subsequent work on GUTs have ignored these threshold effects, perhaps due to the complexity of the Ross approach.

An exception from the late 1980's is the work of Kennedy and Lynn [20], who defined electroweak effective charges similar to the pinch technique charges used in this chapter.

In several papers [21] by Kreuzer, Kummer, and Rebhan, the authors compared the Vilkovisky-DeWitt effective action (VDEA), the mass-shell momentum subtraction scheme(MMOM), and Weinberg's effective gauge theory (EGT). They wrote down explicit formula for the running charges which include analytic threshold behavior for all particles. In calculating predictions from grand unification, they assume asymptotic unification at energies much larger than heavy particles, so that the only threshold corrections from heavy particles come from finite constants which are independent of energy scale or masses. We find similar results in section 3.4.2. Furthermore, we include the possibility of a finite unification scale in section 3.4.4, which leads to more complicated corrections.

In $[22,23]$, the authors include the effects of light supersymmetric scalar and fermion thresholds, although heavy thresholds and gauge bosons virtual effects are not treated. In [24], the authors include both light and heavy threshold corrections, although the treatment of gauge bosons is not complete. In Refs. [25, 26], the authors come to several conclusions similar to ours. However, their definition leads to gauge parameter dependent effective couplings.

\subsection{Physical Renormalization Schemes and Effec- tive Charges}

In order to motivate the re-analysis of supersymmetric unification given in this chapter, we will first discuss some general properties of renormalization schemes in the presence of massive fields and determine a criterion for consistent physical renormalization schemes. These criteria will not be satisfied by the schemes conventionally 
used in unification (and most perturbative calculations), $\overline{M S}$ and $\overline{D R}$, which have persistent threshold and matching errors. Heuristically, these errors can be understood by noting that such schemes implicitly integrate out all masses heavier than the physical energy scale until they are crossed, and then they are "clicked" on with a step function. Of course, integrating out heavy fields is only valid for energies well below their masses. This procedure is problematic since it does not correctly incorporate the finite probability that the uncertainty principle gives for a particle to be pair produced below threshold. Effective charge [11] schemes, derived from physical observables, naturally avoid such errors and are formally consistent.

\subsubsection{A Simple Example}

For the purpose of elucidating the benefits of physical renormalization schemes, we will give a simple toy example using QED with three fermions, $e, \mu$, and $\tau$. Consider the amplitude for the process $e^{-} \mu^{-} \rightarrow e^{-} \mu^{-}$. This can be written as a dressed skeleton expansion, i.e. the dressed tree level graph plus the dressed box diagram plus the dressed double box, etc. The tree level diagram, dressed to all orders in perturbation theory, is equal to the tree level diagram with one modification : the QED coupling $\alpha=\frac{e^{2}}{4 \pi}$ is replaced by the Gell-Man-Low-Dyson effective charge

$$
\alpha\left(Q^{2}\right)=\frac{\alpha}{1+\Pi_{\gamma \gamma}\left(Q^{2}\right)-\Pi_{\gamma \gamma}(0)} .
$$

Hence, from measurements of the cross section, one can measure the effective charge at two different scales, $\alpha\left(Q_{h}^{2}\right)$ and $\alpha\left(Q_{l}^{2}\right)$. Suppose the value of the electron charge is not known, and we are trying to test the predictions of QED. The way to proceed is to use one measurement, say at the low scale $Q_{l}$, as an input to determine $e$. Now the prediction at the high scale $Q_{h}$ is well defined, and represents a test of the theory. More directly, we could just write $\alpha\left(Q_{h}^{2}\right)$ in terms of $\alpha\left(Q_{l}^{2}\right)$, leading to the same prediction. Since the cross section $\sigma_{e^{-} \mu^{-} \rightarrow e^{-} \mu^{-}}\left(Q^{2}\right)$ is proportional to $\left(\alpha\left(Q^{2}\right)\right)^{2}$, we are clearly relating one observable to another. The procedure just outlined is simply an on-shell renormalization scheme if $Q_{l}=0$. More generally, we will refer to such a scheme as an effective charge scheme, since we are writing 
a given observable, here just $\sigma_{e^{-} \mu^{-} \rightarrow e^{-} \mu^{-}}\left(Q_{h}^{2}\right)$ (or $\alpha\left(Q_{h}^{2}\right)$ ), in terms of an effective charge, $\alpha\left(Q_{l}^{2}\right)$, defined from a measurement of the cross section at the scale $Q_{l}$. One could equally well write any observable in terms of this effective charge. Note that this approach to renormalization works for arbitrary scales, even if the low scale lies below some threshold, say $Q_{l}<m_{\tau}$, while $Q_{h}>m_{\tau}$. Decoupling and the smooth "turning on" of the $\tau$ are manifest.

Now we will compare with the results obtained by using the conventional implementation of $\overline{M S}$, which is as follows. First, the cross section is calculated at $Q_{l}$ using the rules of $\overline{M S}$, which allows only the electrons and muons to propagate in loops, since $Q_{l}<m_{\tau}$. Comparing the observed cross section to this result will fix the value of the $\overline{M S}$ coupling for two flavors, $\hat{\alpha}_{2}\left(Q_{l}\right)$. To predict the result of the same experiment at scale $Q_{h}>m_{\tau}$, we need to evolve $\hat{\alpha}_{2}$ to the tau threshold using the two flavor beta function, match with a three flavor coupling, $\hat{\alpha}_{3}$, through the relation $\hat{\alpha}_{2}\left(m_{\tau}\right)=\hat{\alpha}_{3}\left(m_{\tau}\right)$, and then evolve $\hat{\alpha}_{3}\left(m_{\tau}\right)$ to $Q_{h}$ using the three flavor beta function. We will now have a prediction for $\sigma_{e^{-} \mu^{-} \rightarrow e^{-} \mu^{-}}\left(Q_{h}^{2}\right) \propto\left(\alpha\left(Q_{h}^{2}\right)\right)^{2}$. One might expect, from the general principle of $R G$ invariance of physical predictions that this result should be the same as the prediction derived using the physical effective charge scheme above. However, there is a discrepancy arising from the incorrect treatment of the threshold effects in $\overline{M S}$. A detailed discussion of this problem will be given in section 3.7. In any case, the result can be obtained by straightforwardly applying the procedure outlined above. One finds that the ratio of the cross section derived using $\overline{M S}$ with the cross section derived using effective charges, to first order in perturbation theory, is given by

$$
\frac{\hat{\sigma}\left(Q_{h}^{2}\right)}{\sigma\left(Q_{h}^{2}\right)}=1+2 \frac{\alpha\left(Q_{l}\right)}{3 \pi}\left(L_{\tau}\left(Q_{l} / m_{\tau}\right)-5 / 3\right)
$$

where $L_{\tau}$ is a logarithm-like function (the high energy limit is a logarithm) given by

$$
\begin{aligned}
L_{\tau}(Q / m) & =\int_{0}^{1} d x 6 x(1-x) \log \left(1+\frac{Q^{2}}{m^{2}} x(1-x)\right)+5 / 3 \\
& =\left(\beta \tanh ^{-1}\left(\beta^{-1}\right)-1\right)\left(3-\beta^{2}\right)+2,
\end{aligned}
$$


where $\beta=\sqrt{1+\frac{4 m^{2}}{Q^{2}}}$. It satisfies the property $L_{\tau}(0)=5 / 3$, so that there is no discrepancy when the low reference scale $Q_{l}$ is much lower than the tau mass threshold. This reflects the important, but often overlooked, fact that unphysical schemes, such as $\overline{M S}$, are formally consistent only in desert regions where particle masses can be neglected. The error is plotted in Fig.(3.1). Notice that in this example there is an error only for $Q_{l}<m_{\tau}$. However, in the more general case of multiple flavor thresholds, there will be errors from both high and low scales. Similar discrepancies will be found in our analysis of grand unification.

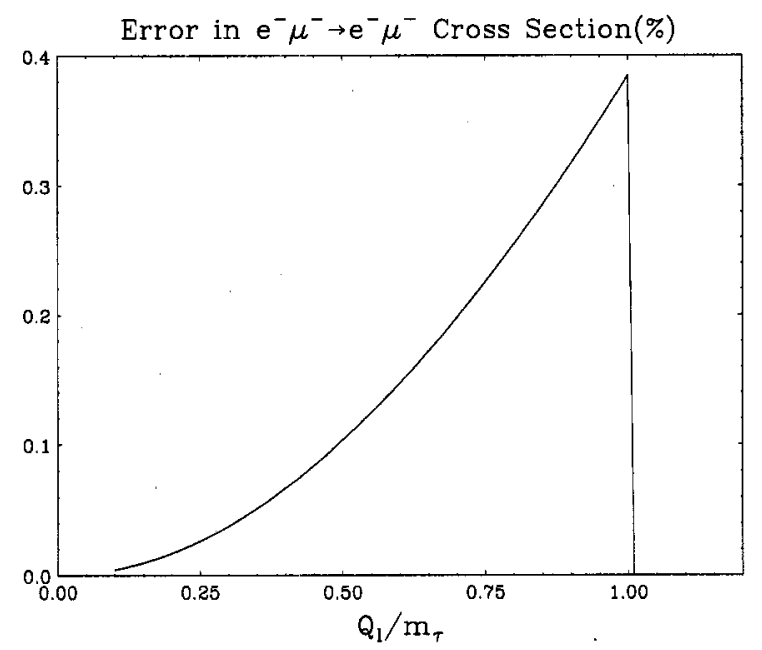

Figure 3.1: The error in the $\overline{M S}$ based prediction for the scattering cross section, $100 \% \times\left(\frac{\hat{\sigma}\left(Q_{h}^{2}\right)}{\sigma\left(Q_{h}^{2}\right)}-1\right)$, plotted against the reference subtraction scale $Q_{l}$ for the choice $\alpha\left(Q_{l}\right) \approx 0.1$.

\subsubsection{General Properties of Effective Charges and Physical Renormalization Schemes}

Effective charges [11] may be defined for any perturbatively calculable observable

$$
\mathcal{O}(Q) \equiv A^{\mathcal{O}}+a_{1}^{\mathcal{O}} \alpha^{\mathcal{O}}(Q)
$$


by absorbing all of the radiative corrections into the effective charge $\alpha^{\mathcal{O}}$. To one-loop order using dimensional regularization (DREG) or dimensional reduction (DRED) in $d=4-2 \epsilon$ dimensions, it is straightforward to show that any unrenormalized effective charge may be parameterized as ${ }^{1}$

$$
\alpha^{\mathcal{O}}(Q)=\alpha_{0}-\frac{\alpha_{0}^{2}}{4 \pi} \sum_{p} \beta_{p}\left(L_{p}\left(Q / m_{p}\right)-\eta_{p}^{\mathcal{O}}\left(Q / m_{p}\right)-C_{U V}+\log \frac{m_{p}^{2}}{\mu^{2}}\right)+\cdots(3.5)
$$

where the sum is over all particles $p$ in the fundamental theory which contribute to the running of the effective charge. In the QED example above, the sum proceeds over $e, \mu, \tau$ and $L_{e}=L_{\mu}=L_{\tau}$, and the function $\eta_{p}^{\mathcal{O}}\left(Q / m_{p}\right)=5 / 3$ is a constant for the simple observable $\mathcal{O}=\sqrt{\sigma_{e^{-} \mu^{-} \rightarrow e^{-} \mu^{-}}}$. In Eq.(3.5), $C_{U V}=\frac{1}{\epsilon}-\gamma_{E}+\log 4 \pi$ is the divergence and associated constants, $\mu$ is the regularization scale, $\alpha_{0}$ is the bare gauge coupling, and $\beta_{p}$ is the contribution of each particle to the one-loop beta function coefficient. The $L_{p}\left(Q / m_{p}\right)$ are logarithmic-like functions which are characteristic of the spin of each particle, and are given exactly in Eq.(3.19). They may be approximated for spacelike momenta to within a few percent ${ }^{2}$ by

$$
L_{p}(Q / m) \approx \log \left(e^{\eta_{p}}+\frac{Q^{2}}{m_{p}^{2}}\right)
$$

and have the limits

$$
L_{p}(Q / m) \stackrel{Q \gg m}{\approx} \log \frac{Q^{2}}{m^{2}}, \quad L_{p}(Q / m) \stackrel{m \gg Q}{\approx} \eta_{p}
$$

where the constants $\eta_{p}$ have values given in Table 3.1. We will see that these constants are of central importance in physical renormalization schemes. These log-like functions characterize the self-energy-like effect of each particle, including the finite

\footnotetext{
${ }^{1}$ This follows from considering the high energy limit and requiring renormalizability. Note also that our parameterization can be easily extended to effective charges which have particles with different masses running together in the loops, and the results are similar. In any case, we will not have use for such charges in this thesis.

${ }^{2}$ To be precise, the approximations reproduce the exact functions $L_{0}, L_{1 / 2}$, and $L_{1}$ (the subscripts refer to the spin of the massive field) with maximum error of $3.5 \%, 0.8 \%$, and $2.2 \%$, respectively, over the entire range of spacelike $Q$.
} 


\begin{tabular}{|c|c|c|c|}
\hline & scalars & fermions & massive gauge bosons \\
\hline \hline$\eta_{p}$ & $8 / 3$ & $5 / 3$ & $40 / 21$ (DRED) or 2(DREG) \\
\hline
\end{tabular}

Table 3.1: Decoupling constants $\eta_{p}$. For massive gauge bosons, this takes different values for dimensional reduction (DRED) and dimensional regularization (DREG).

spread of the wavefunctions near thresholds due to the uncertainty principle, and may be calculated in several different ways, as will be discussed in section 3.3 and Appendix A. Figure 3.2 shows the $L_{p}$ functions for spacelike momenta.

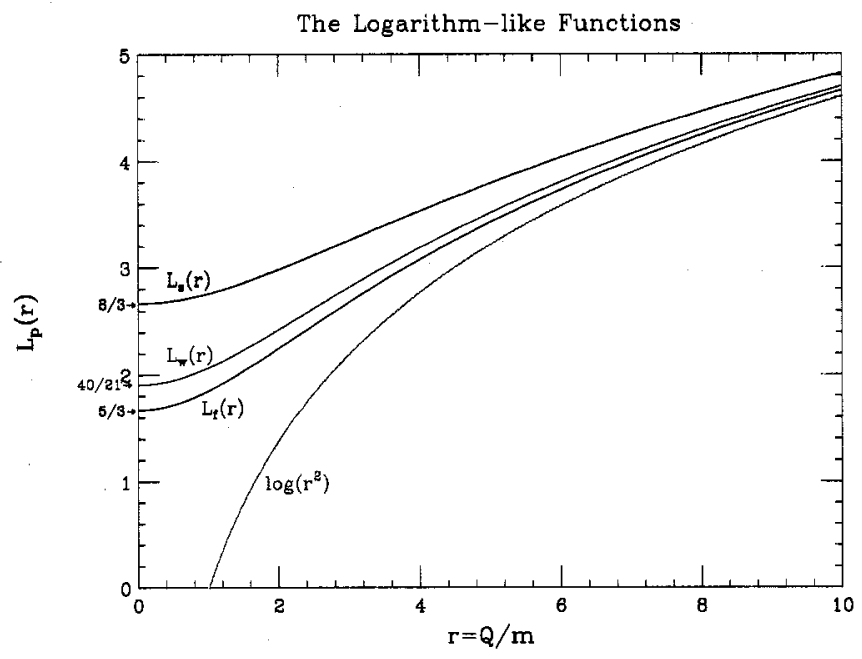

Figure 3.2: The logarithm-like functions for massive particles of spin $0,1 / 2$, and 1 are denoted by $L_{s}, L_{f}$, and $L_{W}$, respectively.

The functions $\eta_{p}^{\mathcal{O}}\left(Q / m_{p}\right)$ are characteristic of each observable, with a nontrivial functional form indicating deviations from self-energy like behavior. For a general observable $\mathcal{O}$, the function $\eta_{p}^{\mathcal{O}}\left(Q / m_{p}\right)$ is nontrivial. The constants $\eta_{p}$ shown in Table 3.1 correspond to a particularly simple and canonical observable, called the pinchtechnique (PT) self-energy-like effective charge, which may be defined by

$$
\eta_{p}^{\mathrm{PT}}\left(Q / m_{p}\right)=\eta_{p}
$$

and Eq.(3.5). This will be discussed in more detail in section 3.3 . 
The effective coupling renormalized in the most general scheme $R$ at 1-loop is

$$
\begin{aligned}
\alpha^{\mathcal{O}}(Q) & =\alpha_{R}\left(Q_{0}\right)-\frac{\left(\alpha_{R}\left(Q_{0}\right)\right)^{2}}{4 \pi} \sum_{p} \beta_{p}\left(L_{p}\left(Q / m_{p}\right)-L_{p}\left(Q_{0} / m_{p}\right)\right. \\
& \left.-\eta_{p}^{\mathcal{O}}\left(Q / m_{p}\right)+\eta_{p}^{R}\left(Q_{0} / m_{p}\right)\right)
\end{aligned}
$$

where the functions $\eta_{p}^{R}\left(Q_{0} / m_{p}\right)$ contain all of the information about the scheme. Here $R$ can be any mathematical scheme for defining the couplings. In the case of $\overline{M S}$, we have $\eta_{p}^{\overline{M S}}\left(Q_{0} / m_{p}\right)=L_{p}\left(Q_{0} / m_{p}\right)-\log \left(Q_{0}^{2} / m_{p}^{2}\right)$ so that only logarithms of the renormalization scale, $Q_{0}$, are subtracted. ${ }^{3}$

It is straightforward to relate observables to each other:

$$
\begin{aligned}
\alpha^{\mathcal{O}_{1}}\left(Q_{1}\right) & =\alpha^{\mathcal{O}_{2}}\left(Q_{2}\right)-\frac{\left(\alpha^{\mathcal{O}_{2}}\left(Q_{2}\right)\right)^{2}}{4 \pi} \sum_{p} \beta_{p}\left(L_{p}\left(Q_{1} / m_{p}\right)-L_{p}\left(Q_{2} / m_{p}\right)\right. \\
& \left.-\eta_{p}^{\mathcal{O}_{1}}\left(Q_{1} / m_{p}\right)+\eta_{p}^{\mathcal{O}_{2}}\left(Q_{2} / m_{p}\right)\right)
\end{aligned}
$$

This satisfies the transitivity property of the physical renormalization group. As before, the sum over $p$ runs over all particles in the fundamental theory which contribute to the effective charges.

For consistency, very massive particles must decouple properly and must not contribute to physical predictions. Taking the $m_{p} \rightarrow \infty$ limit in Eq.(3.9) and Eq.(3.10) yields a fundamental requirement of renormalization schemes and observables:

$$
\eta_{p}^{R}(0)=\eta_{p}^{\mathcal{O}_{1}}(0)=\eta_{p}^{\mathcal{O}_{2}}(0)=\eta_{p}
$$

This consistency requirement holds for all schemes $R$, observables $\mathcal{O}_{1}, \mathcal{O}_{2}$, and for each massive particle $p$. These are universal constants for each spin and are equal to the $\eta_{p}$ given in Table 3.1 above, as can be verified through explicit calculations. Renormalization schemes that satisfy Eq.(3.11) will henceforth be referred to as physical

\footnotetext{
${ }^{3}$ The term in parentheses in Eq.(3.9) becomes $L_{p}\left(Q / m_{p}\right)-\log \left(Q_{0}^{2} / m_{p}^{2}\right)-\eta_{p}^{\mathcal{O}}\left(Q / m_{p}\right)$. Note that in most calculations the first term is taken to be a logarithm and mass corrections are systematically added, in order to approximate the full threshold dependence of $L_{p}\left(Q / m_{p}\right)$. However, the $\log \left(Q_{0}^{2} / m_{p}^{2}\right)$ term does not have the correct threshold dependence, as we will be discussing.
} 
renormalization schemes, and those that do not will be called unphysical renormalization schemes, for reasons that will become clear. In Appendix B a more thorough discussion of the decoupling problem in unphysical schemes is given.

The above discussion implies a unique decoupling limit $(Q / m \rightarrow 0)$ for observables. It is interesting that there is also a restriction on the high energy behavior $(Q / m \rightarrow \infty)$, which holds only for supersymmetric theories and takes the form of a sum rule. It is given by

$$
\frac{\sum_{p \in S} \beta_{p}(G) \eta_{p}^{\mathcal{O}}(\infty)}{\sum_{p \in S} \beta_{p}(G)}=K^{\mathcal{O}},
$$

where $K^{\mathcal{O}}$ is a constant that depends only on the observable, not on the gauge group $G$ or the supermultiplet $S$. The $\eta_{p}^{\mathcal{O}}\left(Q / m_{p}\right)$ are calculated using DRED, otherwise the sum rule is true only for differences $\eta_{p}^{\mathcal{O}_{1}}(\infty)-\eta_{p}^{\mathcal{O}_{2}}(\infty)$ between observables. Further, the result holds for any number of supersymmetries, which may be broken or unbroken at low energies. This can be proven inductively given the result for $\mathcal{N}=1$. It is easy to check using Table 3.1 above and the corresponding result for massless gauge bosons given below Eq.(3.15) that $K^{P T}=2$. The sum rule just expresses the fact that there is no resolution within a supermultiplet at high energies, and is motivated from conformal invariance and physical renormalization scheme invariance. Such a sum rule may provide a powerful link between the contributions of various spin fields to any observable, particularly if a multi-loop or non-perturbative generalization was found.

\subsection{The Canonical Physical Effective Charges of the MSSM}

The difficulties associated with unphysical schemes (see Appendix B) are circumvented in physical renormalization schemes (PRS) based on effective charges. The consistency conditions which must be satisfied by PRS's, given in Eq.(3.11), are trivially satisfied by the pinch-technique effective charge (Eq.(3.8)). This is the topic to which we now turn. 
For any observable $\mathcal{O}$, we define an effective charge scheme $R^{\mathcal{O}}$, by

$$
\eta_{p}^{R^{\mathcal{O}}}\left(Q / m_{p}\right)=\eta_{p}^{\mathcal{O}}\left(Q / m_{p}\right)
$$

which, after using Eq.(3.9), is equivalent to

$$
\alpha^{\mathcal{O}}(Q)=\alpha_{R^{\mathcal{O}}}(Q)
$$

thus motivating the terminology "effective charge". Here $R^{\mathcal{O}}$ is the physical subset of all possible mathematical schemes. The canonical example for using an effective charge as a scheme is furnished in QED by the Gell-Mann-Low-Dyson charge, which can be measured directly from scattering experiments. The extension of this concept to non-abelian gauge theories is non-trivial [29], due to the self interactions of the gauge bosons which make the usual self-energy gauge dependent. However, systematically implementing the Ward identities of the theory allows one to project out the unique self-energy of each physical particle, resulting in a self-energy that is gauge independent, may be resummed to define an effective charge, and may be related via the optical theorem to appropriate cuts of differential cross sections. The algorithm for performing the calculation at the diagrammatic level is called the pinch-technique (PT) [30][31][32][33] ${ }^{4}$.

The procedure is illustrated in Fig.(3.3) QCD, where momentum factors from internal gauge boson lines or vertices combine with gamma matrices to cancel internal fermion propagators, yielding a gluon self-energy-like graph. This is then added to the usual self-energy to yield the full PT self-energy.

The PT procedure is unambiguous at one loop and is merely an application of the Ward identities of the theory, which becomes more transparent in a dispersive derivation from physical cross sections $\sigma(q \bar{q} \rightarrow g g)$ [34] (see [33] for such a construction for the electroweak sector). The generalization of the pinch technique to higher loops has recently been investigated [35, 36, 37, 38, 39]. In the work of Binosi and Papavassiliou $[37,38,39]$, the authors prove the consistency of the pinch technique to

\footnotetext{
${ }^{4}$ The interested reader should consult these references for a more detailed discussion of the pinchtechnique algorithm, which will only be sketched here.
} 
all orders in perturbation theory, suggesting how to define the QCD and electroweak effective charges at higher orders. Chapter 6 of this thesis presents a detailed two-loop calculation of the PT effective charge.

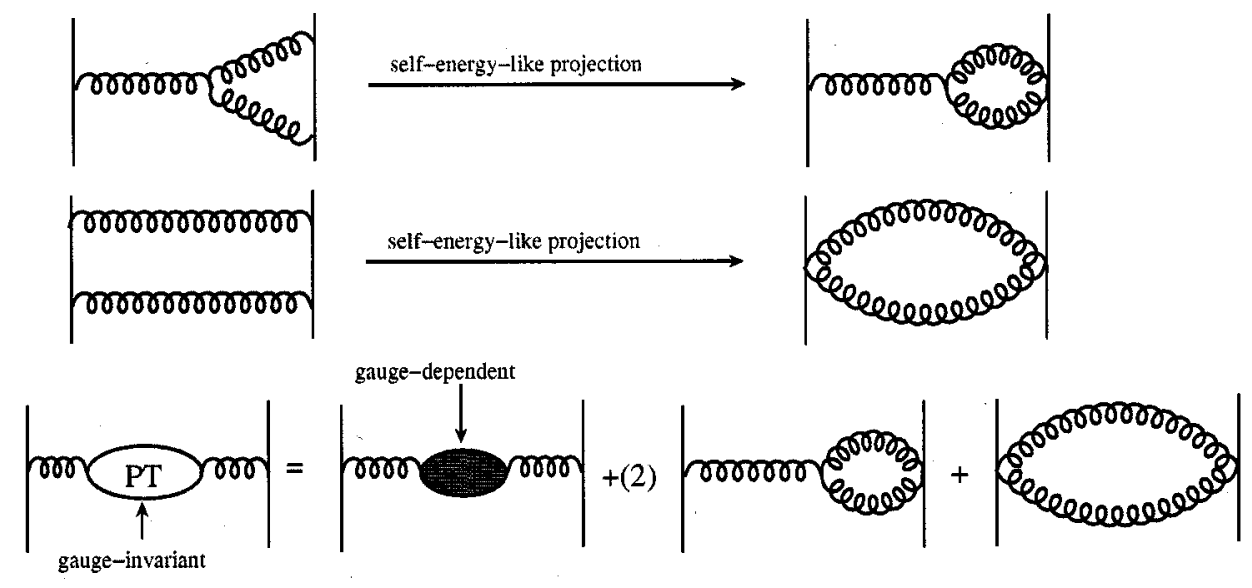

Figure 3.3: Pinch-technique for QCD at 1 loop. The unique gluonic self-energylike projection of the vertex and box graphs yield terms which must be added to the conventional self-energy to get the PT effective charge.

Re-summing the PT self-energy leads to the PT charge, $\widetilde{\alpha}$, which can be written in terms of the bare coupling $\alpha_{0}$ for arbitrary gauge theory, broken or unbroken, as ${ }^{5}$

$$
\tilde{\alpha}(Q)=\alpha_{0}-\frac{\alpha_{0}^{2}}{4 \pi} \sum_{p} \beta_{p}\left(L_{p}\left(Q / m_{p}\right)-\eta_{p}-C_{U V}+\log \left(m_{p}^{2} / \mu^{2}\right)\right)+\cdots
$$

where $\eta_{p}^{P T}\left(Q / m_{p}\right)=\eta_{p}$ are the constants given in Table 3.1 for massive fields and $\eta_{g}=64 / 33$ (DRED) or 67/33(DREG) for massless spin 1 fields ${ }^{6}$. The fact that these $\eta_{p}^{\mathcal{O}}(Q / m)$ functions are constants is what makes the $P T$ observable the most simple and natural choice for defining an effective charge scheme. More general physical

\footnotetext{
${ }^{5}$ If particles of different mass propagate together in the loops, this formula is modified.

${ }^{6}$ We will use ' $W^{\prime}$ or ' 1 ' subscripts to denote massive spin 1 fields and a ' $g$ ' subscript for massless spin fields. The constants $64 / 33$ and $40 / 21$ (for DRED) are related straightforwardly. In general, for a massive gauge boson $W$ in the representation $R$ of group $G$

$$
\beta_{W}=\frac{11}{3} C(R)-\frac{1}{6} C(R)=\frac{7}{2} C(R)
$$


effective charge schemes (see Eqs. $(3.9,3.10,3.11)$ ) have more complicated running due to the $\eta_{p}^{\mathcal{O}}\left(Q / m_{p}\right)$ terms.

Here will use dimensional reduction (DRED), rather than dimensional regularization (DREG) in defining the PT coupling $\widetilde{\alpha}(Q)$, since this is the appropriate choice for analyzing supersymmetric unification. We will let $\overline{P T}$ stand for the renormalization scheme associated to the PT observable regularized using DRED.

Using the above results, it is straightforward to write down the effective charges for the standard model through

$$
\begin{aligned}
\widetilde{\alpha_{1}}\left(Q^{2}\right) & =\frac{5}{3} \frac{\widetilde{\alpha}\left(Q^{2}\right)}{1-\widetilde{s}^{2}\left(Q^{2}\right)} \\
\widetilde{\alpha_{2}}\left(Q^{2}\right) & =\frac{\widetilde{\alpha}\left(Q^{2}\right)}{\widetilde{s^{2}}\left(Q^{2}\right)} \\
\widetilde{\alpha_{3}}\left(Q^{2}\right) & =\widetilde{\alpha_{s}}\left(Q^{2}\right),
\end{aligned}
$$

where the effective couplings $\widetilde{\alpha}$ and $\widetilde{s}^{2}$ are defined from PT self-energies $\widetilde{\Pi}_{\gamma \gamma}$ and $\widetilde{\Pi}_{\gamma Z}$, respectively [33], as is detailed in Appendix A. It is convenient to write $\widetilde{\alpha_{1}}$ and $\widetilde{\alpha_{2}}$ in terms of $\widetilde{\alpha}$ and $\widetilde{s}^{2}$ since the latter contain the contributions from the mass eigenstate fields. One could use Eq.(3.15) directly, although the Higgs sector requires care.

Several subtleties should be addressed before the numerical values of the $\overline{P T}$ couplings are given.

An important difference between the physical effective charges and the unphysical $\overline{M S}$ couplings is a distinction between timelike and spacelike momenta. In conventional approaches, thresholds are treated in a step function approximation, and hence the running is always logarithmic. The analytic continuation from spacelike to timelike momenta is trivial, yielding $i \pi$ imaginary terms on the timelike side. Thus, the real parts of such couplings are the same modulo three loop $(i \pi)^{2}$ corrections. In contrast, the $\overline{P T}$ couplings on timelike and spacelike sides have considerable differences at one-loop. To see this we need the exact expressions for the logarithmic-like and

$$
\eta_{W}=\frac{1}{\beta_{W}}\left(\frac{11}{3} C(R)\left(\frac{64}{33}\right)-\frac{1}{6} C(R)\left(\frac{8}{3}\right)\right)=\frac{40}{21} .
$$


functions of a particle of spin $s$, which can be written as

$$
L_{s}(Q / m)=2\left[\left(\beta \tanh ^{-1}\left(\beta^{-1}\right)-1\right)\left(\frac{4 S^{2}-\beta^{2}}{4 S^{2}-1}\right)+1\right],
$$

where $S^{2}=s(s+1)$ is the total spin squared eigenvalue, $\beta=\sqrt{1+\frac{4 m^{2}}{Q^{2}}}$, and the momenta is spacelike $\left(Q^{2}>0\right)$. This formula is merely a compact way to write the results for massive spin $0,1 / 2$, and 1 fields, and has not been explicitly verified for higher spins. For example, $L_{s=1}$ is calculated from the sum of the usual gauge boson self interaction loop, the ghost loops, the appropriate loops of Goldstone bosons that are eaten, and the pinched parts of the vertex and box graphs (see Appendix A for details). In contrast, $L_{s=1 / 2}$ is simply related to the usual fermion vacuum polarization graph, and $L_{s=0}$ comes from the usual scalar contribution to the gauge boson selfenergy (two diagrams). It is interesting that such a simple compact form is obtained, considering the seemingly different derivations of the three $L_{s}$ functions. This may suggest a more efficient formulation of the perturbative dynamics of quantum fields that treats the various spins in a unified manner [40]. Notice that

$$
\lim _{m \rightarrow \infty} L_{s}(Q / m)=\frac{8}{3}\left[\frac{1-3 s(s+1)}{1-4 s(s+1)}\right],
$$

corresponding to the results of Table 3.1. The analytic continuation of Eq.(3.19) to timelike momenta below threshold, $0<q^{2}=-Q^{2}<4 m^{2}$, is obtained by replacing

$$
\beta \rightarrow i \bar{\beta}, \text { where } \bar{\beta}=\sqrt{\frac{4 m^{2}}{q^{2}}-1} \text {, and } \tanh ^{-1}\left(\beta^{-1}\right) \rightarrow-i \tan ^{-1}\left(\bar{\beta}^{-1}\right) .
$$

Above threshold, $q^{2}>4 m^{2}$, one should replace

$$
\tanh ^{-1}\left(\beta^{-1}\right) \rightarrow \tanh ^{-1}(\beta)-i \frac{\pi}{2} \text { where } \beta=\sqrt{1-\frac{4 m^{2}}{q^{2}}} .
$$

From these results it is clear that significant differences will arise between the spacelike 
and timelike couplings evaluated at scale $\pm M_{Z}^{2}$, mainly due to the $W$-boson threshold asymmetry.

As has been discussed, another distinction of effective couplings is that they are automatically sensitive to light SUSY thresholds near $M_{Z}$, since the $L_{s}$ functions are not zero below threshold (on the spacelike side nor on the timelike side). The effects of light SUSY thresholds on the values of the couplings at the Z-pole will depend on the method of extraction from the data. The key question is whether or not the light sparticles are implicitly included in the measured values of the couplings at $M_{Z}$. For $\widetilde{\alpha}\left(M_{Z}\right)$, which is extracted by running the precisely known fine structure constant from $Q=0$ to $M_{Z}$, we should include corrections from virtual effects of sparticles (with model-dependent mass), in the self-energy term $\Pi_{\gamma \gamma}\left(M_{Z}\right)$. However, these threshold corrections will cancel in any unification prediction, since then one is essentially running from $Q=0$ to $Q=M_{G U T}$ and the light SUSYs are either fully decoupled or fully turned on. For the strong and weak couplings we use data from the Z-pole, and thus no unknown sparticle thresholds must be accounted for since they are already implicitly contained in the measured values. When these couplings are run to the unification scale the induced light threshold corrections will not cancel. Of course, linear combinations of the electromagnetic and weak couplings (Eq.(3.18)) are used for unification, which complicates the matter further, since different methods of extraction are used for each. It would be unpleasant to quote a different value of $\widetilde{\alpha}^{-1}\left(M_{Z}\right)$ for each different SUSY spectra considered. However, this approach has the advantage that the values of the couplings used are the values that one would directly measure in an experiment at $M_{Z}$ if a given sparticle spectrum were the correct one. For convenience, we will quote the QED coupling extracted assuming a fully decoupled SUSY, When calculating detailed unification predictions in given models, however, the appropriate terms will be included in the determination of $\widetilde{\alpha}^{-1}\left(M_{Z}\right)$. It should be emphasized that the above complications are only numerically significant for light sparticle spectra.

The initial values may be extracted from experimental data and are given in Table 3.2 , where spacelike and timelike effective couplings are denoted with a ' $+{ }^{\prime}$ and ' - ', respectively. The $\overline{M S}$ and $\overline{D R}$ couplings are on the timelike side. 


\begin{tabular}{|c|c|c|c|c|}
\hline & $\overline{M S}$ & $\overline{D R}$ & $\bar{P}_{+}$ & $\bar{P}_{-}$ \\
\hline \hline$\alpha^{-1}\left(M_{Z}\right)$ & $127.934(27)$ & $127.881(27)$ & $129.076(27)$ & $128.830(27)$ \\
\hline$s^{2}\left(M_{Z}\right)$ & $0.23114(20)$ & $0.23030(20)$ & $0.23130(20)$ & $0.22973(20)$ \\
\hline$\alpha_{3}\left(M_{Z}\right)$ & $0.118(4)$ & $0.119(4)$ & $0.140(5)$ & $0.140(5)$ \\
\hline
\end{tabular}

Table 3.2: Coupling values at $M_{Z}$. For timelike couplings only the real parts are given.

See Appendix A for detailed formulas for the effective couplings.

Notice that the value of the $\overline{P T}_{+}$electromagnetic inverse coupling, $\widetilde{\alpha}^{-1}\left(M_{Z}\right)=$ 129.076(27), does not correspond to the usual value of about 128.968(27). This discrepancy arises because $\widetilde{\alpha}^{-1}\left(M_{Z}\right)$ includes the virtual effects of $W^{+} W^{-}$loops, whereas the usual construction of $\alpha_{\mathrm{QED}}\left(M_{Z}\right)$ entirely ignores the virtual effects of the massive gauge bosons. The proximate cause of this consistent oversight in the literature is the difficulty in extracting a gauge invariant self-energy-like contribution to the running couplings for non-abelian theories, a problem which is resolved through the pinch technique, in particular, and more generally, in any effective charge scheme. While it is simply a matter of convention whether one includes the virtual effects of the massive gauge bosons in the running coupling or includes them elsewhere, it is clear that the former approach, which is used here, yields a coupling which more accurately reflects the strength of the electromagnetic force. Similar comments apply to the weak mixing angle.

It should be emphasized that although we have chosen to discuss a particular physical renormalization scheme (PRS), it will be shown in the next section that all predictions associated with unification are PRS invariant, as they should be. However, a definite scheme must be chosen for explicit calculations, and the $\overline{P T}$ scheme is the simplest choice. As expected, we will find that PRS invariance does not extend to unphysical schemes such as $\overline{M S}$ or $\overline{D R}$, because of errors associated with the incorrect treatment of light and heavy thresholds (see Appendix B). 


\subsection{Unification in Physical Renormalization Schemes}

Now we are ready to discuss unification. In section 3.4.1, we will consider only the light spectrum given by the standard model fields and their $N=1$ superpartners. This gives a model-independent starting point for discussing unification, and makes clear exactly what model dependent heavy threshold corrections are needed for consistency with the unification hypothesis. New light threshold corrections, in addition to the usual light mass corrections, are evident, although they are numerically important for only a small range of parameter space corresponding to light sparticles. In section 3.4 .2 , asymptotic unification is introduced, leading to substantial qualitative changes in the usual picture of gauge unification. This particular choice of unification boundary conditions will lead to corrections from logarithms of superheavy mass ratios, just as would be obtained by implementing $\overline{D R}$ with the step function approximation. This sheds light on the nature of the approximation of the $\overline{D R}$ approach. In section 3.4.3, an effective unification scale is derived that is considerable higher than the usual unification scale. In section 3.4.4, more general non-asymptotic boundary conditions are considered, and the new non-trivial thresholds corrections are found to be important.

In performing the analysis, the exact analytic one-loop formulas discussed in section 3.3 will be used, as well as the leading two-loop corrections. The analytic mass dependent two-loop corrections are not known, but these can be estimated to be numerically small and well within the error bars, and hence can be neglected [41].

We will treat the SUSY spectrum as entirely arbitrary, rather than assume a particular model or theoretical bias. The advantage of this approach is that importance of various spectra parameters becomes transparent, and irrelevant details can be ignored.

\subsubsection{The (in)validity of Neglecting Heavy Thresholds}

In this subsection only, heavy thresholds will be entirely neglected.

The usual test of unification is to predict $\alpha_{3}\left(M_{Z}\right)$ contingent upon unification. Compared with the conventional $\overline{D R}$ framework, we expect to see improvements 
due to the correct treatment of light thresholds. To be precise, the corrections we are discussing are to the difference between the $\widetilde{\alpha}_{3}\left(M_{Z}\right)$ prediction obtained from the following two methods: (a)using the $\overline{P T}_{+}$scheme throughout, (b) using $\overline{D R}$ (with the artificial decoupling and theta function treatment of light thresholds) to predict $\hat{\alpha}_{3}\left(M_{Z}\right)$, which is then translated to a prediction for $\widetilde{\alpha}_{3}\left(M_{Z}\right)$. Both approaches capture the leading light threshold effects, which appear as logarithms of light masses. The additional corrections in the $\overline{P T}$ scheme are from what we will call analytic light threshold corrections, since they arise from correctly and smoothly interpolating between thresholds. These are largest when there are light supersymmetric partners near or below $M_{Z}$. For most values of the sparticle masses, they fall inside the error bars. However, such corrections may become more important as the experimental values of the couplings are determined more precisely. The exact form of the new corrections will be shown explicitly in section 3.4.2, Eqs.(3.27,3.31).

Now let us compare the $\overline{P T}$ unification predictions with experiment. The predictions for the $\overline{P T}$ strong coupling, $\widetilde{\alpha_{3}}$, (obtained through method (a)) are displayed in Fig.[3.4] against the SUSY scale and in Fig.[3.5] against the mass ratio of the gluino and wino. These are the two SUSY spectrum parameters to which the $\widetilde{\alpha_{3}}$ prediction is most sensitive.

Only light gluino scenarios with $m_{\tilde{g}} \lesssim m_{\tilde{w}}$, are able to correctly predict the strong coupling for natural SUSY scales (less than about a TeV). However, it is generally expected that the gluino is several times heavier than the wino for most realistic models of supersymmetry breaking and spectra. Hence, we reproduce the known result [42] that, at two loops and neglecting heavy thresholds, gauge coupling unification fails by several standard deviations. Except for the light gluino escape route, this points to the need for large heavy threshold corrections if unification is to be achieved.

\subsubsection{Heavy Thresholds and Asymptotic Unification}

Henceforth, the complete heavy threshold behavior will be included in the running of the effective couplings. The form of the subsequent corrections will depend on the particular unification boundary conditions that are chosen, and the numerical values 


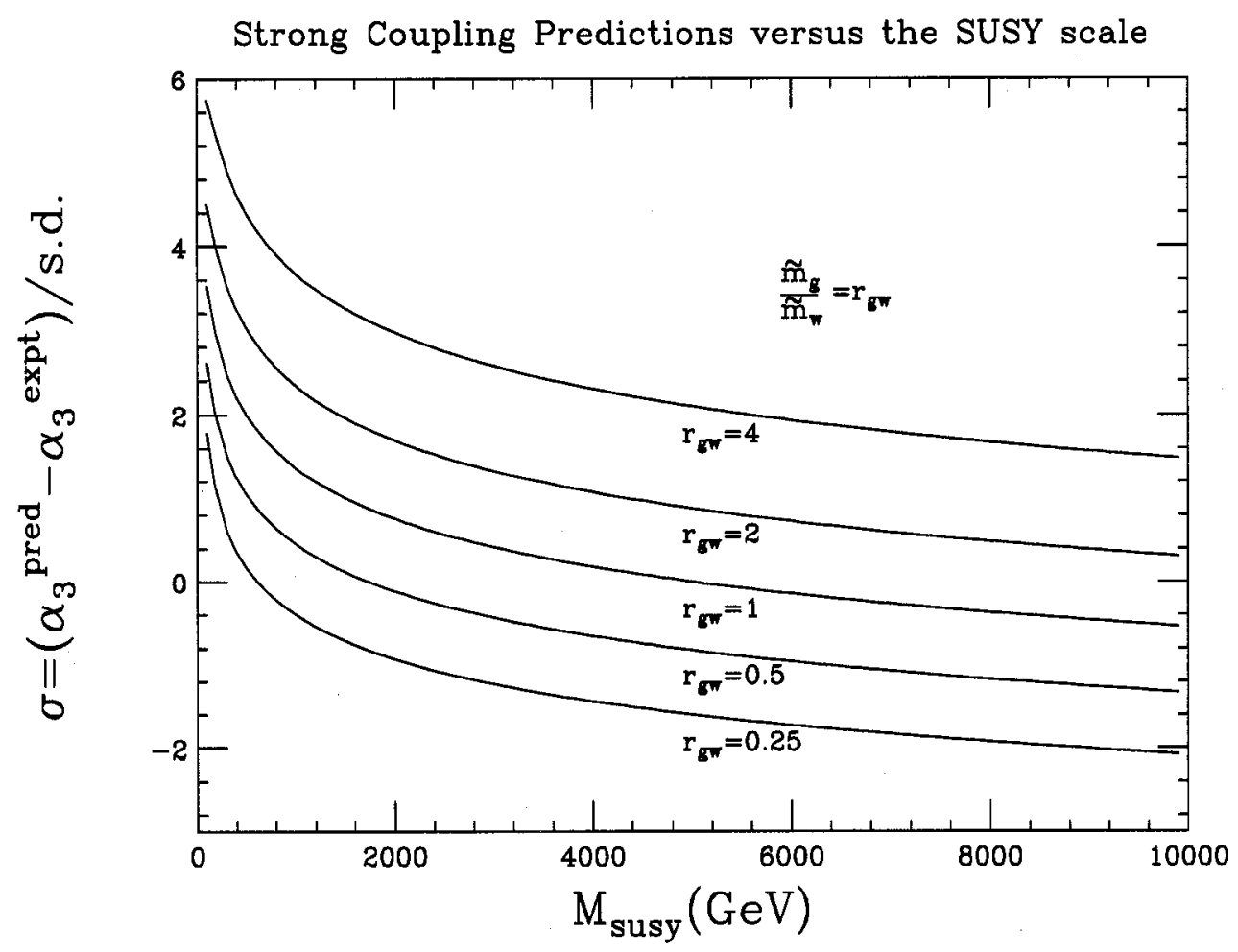

Figure 3.4: The error in the prediction for $\widetilde{\alpha_{3}}\left(M_{Z}\right)$ is plotted against the typical SUSY mass scale, with different lines corresponding to values of the ratio of the gluino mass to the wino mass. The relative mass spectrum is roughly the same as most sparticle spectrum models, including supergravity models, with $M_{s}$ setting the overall scale. The experimental standard deviation, s.d., is 0.0055 for the PT strong coupling.

of the corrections will depend on the details of the GUT model. In this section we will choose the simplest boundary conditions, since it will reproduce known results. Later, more general cases will be considered.

Generally, there are four parameters which specify the unification boundary conditions. These are the unification scale, $M_{U}$, and the values of the couplings at that scale, $\widetilde{\alpha}_{i}\left(M_{U}\right)$ for $i=1,2,3$. For our purposes, we will always assume standard normalizations and take the couplings to be equal at some scale. In this case, the only free parameter is $M_{U}$. The two distinct cases are for finite $M_{U}$ and infinite $M_{U}$. The so-called asymptotic unification considered in this section corresponds to the latter choice, namely $M_{U} \rightarrow \infty$ and $\alpha_{1}^{-1}\left(M_{U}\right)=\alpha_{2}^{-1}\left(M_{U}\right)=\alpha_{3}^{-1}\left(M_{U}\right)$. The asymptotic unification conditions would be appropriate if the standard model group $G_{S M}$ is embedded 


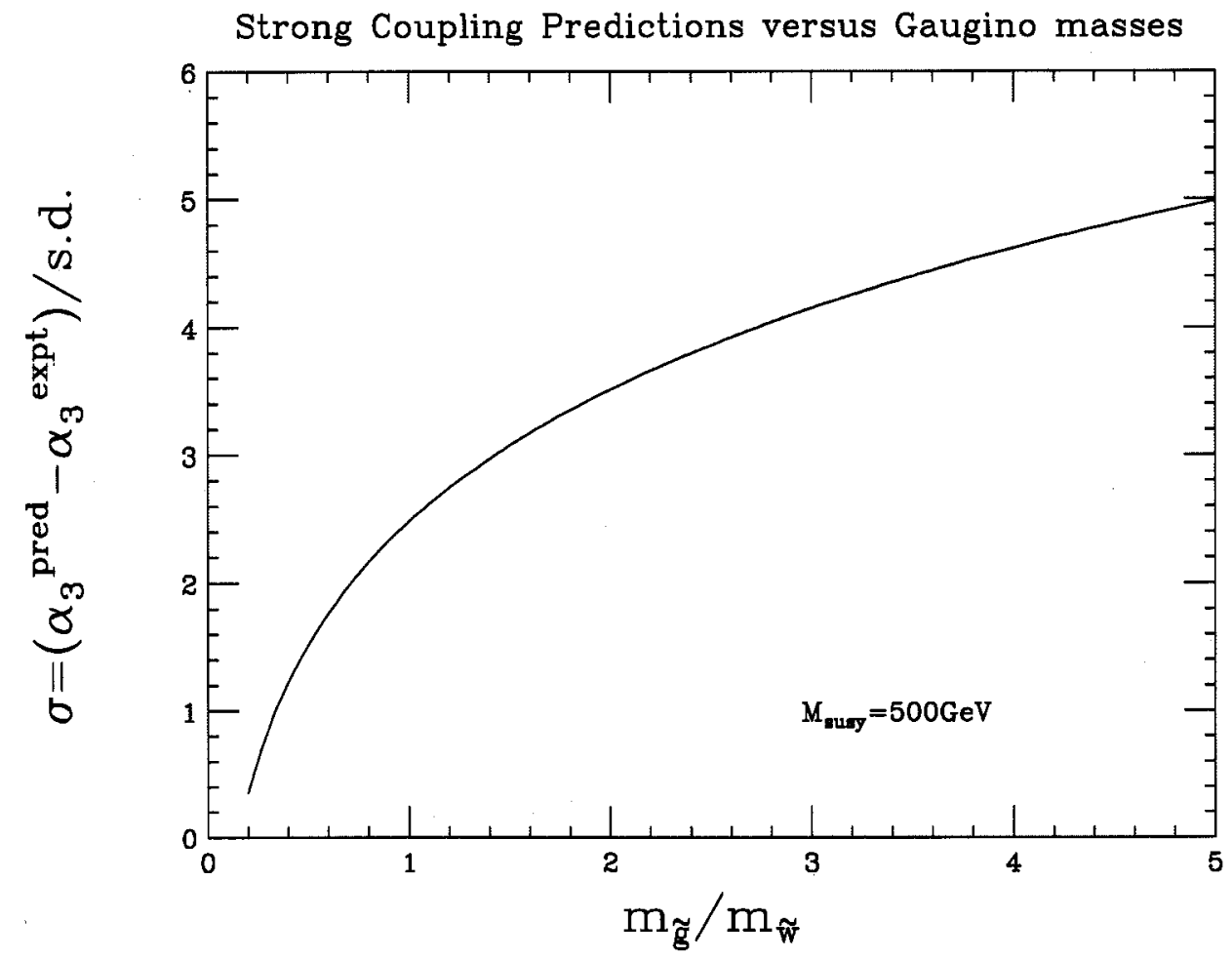

Figure 3.5: The error in the prediction for $\widetilde{\alpha_{3}}\left(M_{Z}\right)$ is plotted against the ratio of the gluino mass to the wino mass, which is the sparticle spectrum parameter to which $\widetilde{\alpha_{3}}\left(M_{Z}\right)$ predictions are most sensitive. The spectrum is fully specified by the ratio and $\sqrt{m_{\tilde{g}} m_{\widetilde{w}}}=500 \mathrm{GeV}=M_{\text {susy }}$, where $M_{\text {susy }}$ is the mass of all other sparticles.

in a simple Lie group $G$ which is fully restored before gravitational or other string interactions become relevant, and neglecting any other exotic phenomena. Hence, this choice is somewhat simple and naive, but it is very instructive.

We will find that asymptotic unification reproduces the same heavy threshold corrections which can be obtained by unphysical renormalization schemes $(\overline{D R})$ with finite unification scale. The reason is that in taking $M_{U} \rightarrow \infty$, one is essentially looking at an observable (the unification requirement) in a desert region, which, as we have seen, unphysical schemes are capable of treating without error. At first sight, it may seem strange that the infinite unification scale predictions of physical schemes correspond to finite unification scale predictions of unphysical schemes. However, this is dictated by the nature of unphysical schemes where masses are turned on and off 
with a step function.

The paradigmatic improvement over conventional methods is summarized in Fig.[3.6], where asymptotic unification of the couplings occurs at very large energy. For demonstrative purposes, the parameters are chosen so that unification occurs.

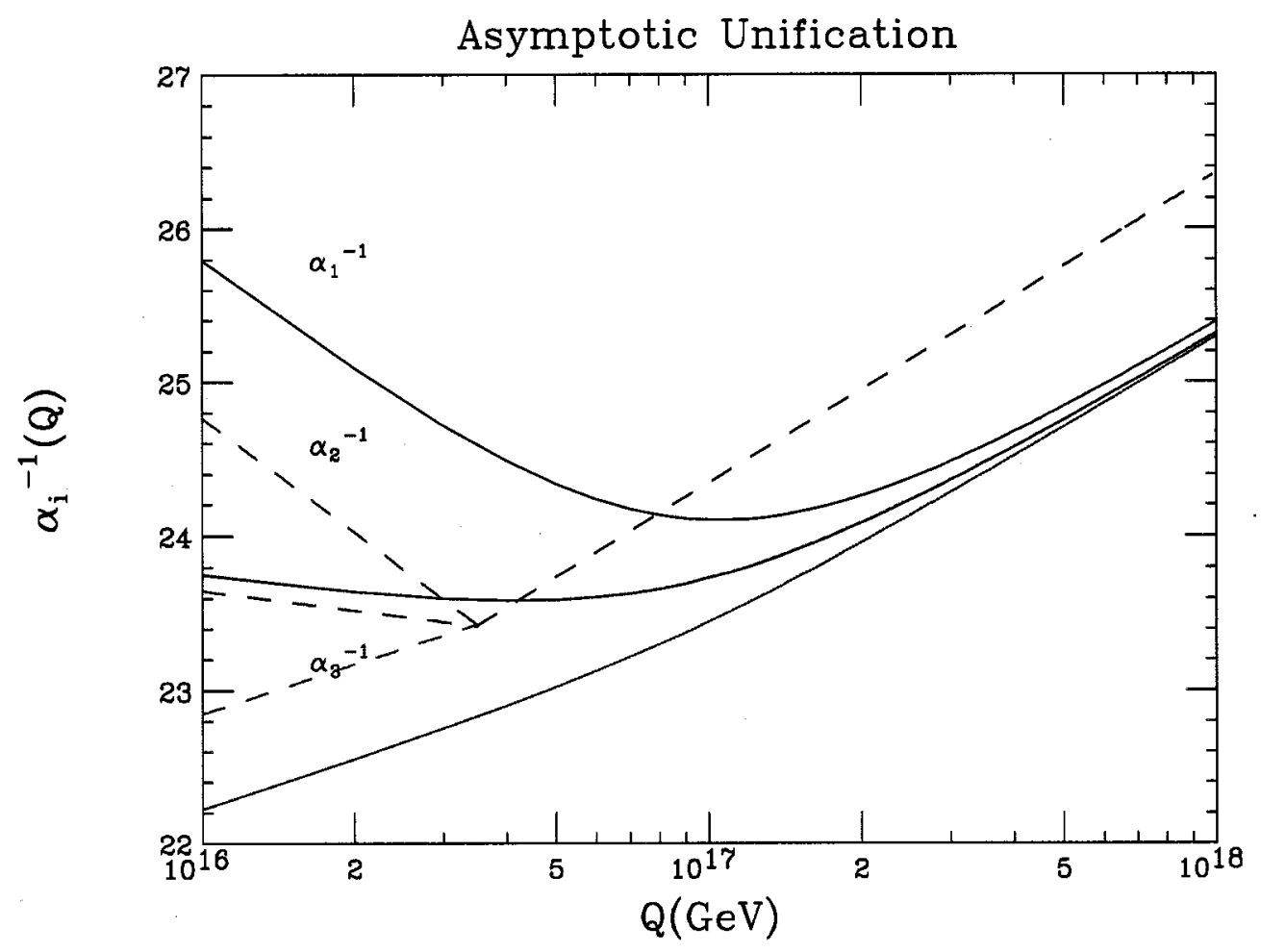

Figure 3.6: Asymptotic Unification. The solid lines are the analytic $\overline{P T}$ effective couplings, while the dashed lines are the $\overline{D R}$ couplings. For illustrative purposes, $\alpha_{3}\left(M_{Z}\right)$ has been chosen so that unification occurs at a finite scale for $\overline{D R}$ and asymptotically for the $\overline{P T}$ couplings. Here $M_{S U S Y}=200 \mathrm{GeV}$ is the mass of all light superpartners except the wino and gluino which have values $\frac{1}{2} m_{\tilde{g}}=M_{S U S Y}=2 m_{\tilde{w}}$. For illustrative purposes, we use $S U(5)$.

Now let us derive the analytic formulae for the unification predictions. We will discuss the most general case of an $N=1$ supersymmetric $G_{S M}=U(1)_{Y} \otimes S U(2)_{L} \otimes S U(3)_{C}$ embedded in a larger gauge group, $G$, using any physical $\mathrm{RN}$ scheme (all others are inconsistent), which we label by its associated observable, $\mathcal{O}$. 
In general the running of the couplings can be expressed in the form

$$
\alpha_{\mathcal{O}_{i}}^{-1}(Q)=\alpha_{\mathcal{O}_{i}}^{-1}\left(Q_{0}\right)+\widetilde{\Pi}_{i}^{\mathcal{O}}\left(Q, Q_{0}\right)-\theta_{i}\left(Q, Q_{0}\right)
$$

where the two-loop corrections ${ }^{7}$ are contained in $\theta_{i}\left(Q, Q_{0}\right)$, and we have defined

$$
\widetilde{\Pi}_{i}^{\mathcal{O}}\left(Q, Q_{0}\right)=\frac{1}{4 \pi} \sum_{p \in G} \beta_{i}^{(p)}\left(L_{p}\left(Q / m_{p}\right)-L_{p}\left(Q_{0} / m_{p}\right)-\eta_{p}^{\mathcal{O}_{i}}\left(Q / m_{p}\right)+\eta_{p}^{\mathcal{O}_{i}}\left(Q_{0} / m_{p}\right)\right)
$$

which contains all of the one loop corrections. Now we separate the sums over the light and heavy spectra, $L=G_{S M}\left(G_{S M}\right.$ means the standard model fields plus SUSY partners) and $H=G-G_{S M}$, take $Q_{0}=M_{Z}$, and let $Q=M_{U}$ be some energy much larger than the mass of all fields, including the heavy fields; i.e. $M_{U} \gg m_{p} \forall p \in L+H$. The functions $\widetilde{\Pi}_{i}^{\mathcal{O}}$ can then be written as

$$
\widetilde{\Pi}_{i}^{\mathcal{O}}\left(M_{U} \rightarrow \infty, M_{Z}\right)=\beta_{G} l_{U}-\Delta_{i}^{L}-\delta_{i}^{H}-\beta_{i}^{H} l_{X}-S_{L, i}^{\mathcal{O}}(\infty)+S_{L, i}^{\mathcal{O}}\left(M_{Z}\right)-S_{H, i}^{\mathcal{O}}(\infty)
$$

where $\beta_{G}=\sum_{p \in G} \beta_{p}, l_{U}=\frac{1}{2 \pi} \log \frac{M_{U}}{M_{Z}}, l_{X}=\frac{1}{2 \pi} \log \frac{M_{X}}{M_{Z}}$,

$$
\begin{gathered}
S_{L, i}^{\mathcal{O}}(Q)=\sum_{l \in L} \frac{1}{4 \pi} \beta_{i}^{(l)} \eta_{l}^{\mathcal{O}}\left(Q / m_{l}\right), \\
\Delta_{i}^{L}=\sum_{l \in L} \frac{1}{4 \pi} \beta_{i}^{(l)}\left(L_{l}\left(\frac{M_{Z}}{m_{l}}\right)-\log \frac{M_{Z}^{2}}{m_{l}^{2}}\right),
\end{gathered}
$$

and

$$
\delta_{i}^{H}=\sum_{h \in H} \frac{1}{4 \pi} \beta_{i}^{(h)} \log \frac{m_{h}^{2}}{M_{X}^{2}} .
$$

The exact one-loop analytic light threshold corrections are contained in $\Delta_{i}^{L}$, while the heavy threshold splittings are contained in $\delta_{i}^{H}$, with some arbitrarily chosen heavy mass $M_{X}$ which is conveniently taken to be the mass of heavy gauge bosons.

It is useful to verify that predictions for $l_{X}$ and $\alpha_{3}\left(M_{Z}\right)$ are invariant under the choice of physical renormalization scheme. In performing the calculation, one must

\footnotetext{
${ }^{7}$ see Appendix B for the details
} 
use the fact that the $\eta_{p}^{\mathcal{O}}$ functions do not depend on the gauge group or representation of $p$, only the spin. These are necessary (but not sufficient) conditions for the sum rule in Eq.(3.12). This scheme equivalence does not extend to unphysical schemes such as $\overline{D R}$, though the errors are quantifiable.

Due to the physical renormalization scheme invariance, we may choose the simplest scheme, which is the $\overline{P T}$ scheme discussed earlier. Because the $\eta_{p}^{\overline{P T}}$ functions are constants equal to $\eta_{p}=L_{p}(0)$, the expressions for the unification predictions are simple and compact when written in terms of the $\overline{P T}$ charges $\widetilde{\alpha}_{i}$.

From $\widetilde{\alpha}_{1}\left(M_{U}\right)=\widetilde{\alpha}_{2}\left(M_{U}\right)$, the heavy gauge boson mass, $M_{X}$, is given by

$$
\frac{\log \left(\frac{M_{X}}{M_{Z}}\right)+1}{2 \pi}=\frac{\widetilde{\alpha}_{2}^{-1}\left(M_{Z}\right)-\widetilde{\alpha}_{1}^{-1}\left(M_{Z}\right)+\Delta_{12}}{\beta_{12}}
$$

where $\Delta_{12}=\Delta_{1}-\Delta_{2}, \beta_{12}=\beta_{1}-\beta_{2}$, etc., and $\Delta_{1}=\Delta_{1}^{L}+\delta_{1}^{H}+\theta_{1}$. Notice that $M_{X}$ can be determined explicitly only for the (unlikely) case of a degenerate heavy spectrum when $\delta_{i}^{H}=0$, otherwise the expression is transcendental in $M_{X}$. In the degenerate case, the gauge boson mass $M_{X}=M_{U}^{t} / e$ is equal to the unification scale determined by entirely neglecting heavy thresholds(denoted by $\not H$ ), divided by $e=2.71828 \ldots$. This result relies on use of the sum rule in Eq. (3.12) which gives rise to the $1 / 2 \pi$ term on the LHS of Eq.(3.29). The generalization to arbitrary physical renormalization scheme is $M_{X}=M_{U}^{t} e^{-K^{\mathcal{O}} / 2}$, where $K^{\mathcal{O}}$ is defined in Eq.(3.12). Neglecting the light and heavy analytic non-logarithmic threshold corrections, the gauge boson mass prediction is the same as the unification scale prediction in the $\overline{D R}$ scheme. Also, the 'unification' scale $M_{U}^{f}$ depends on the particular scheme, which makes sense since different schemes correspond to different observables. In contrast, $M_{X}$ is scheme independent.

The strong coupling prediction is

$$
\widetilde{\alpha}_{3}^{-1}\left(M_{Z}\right)=\widetilde{\alpha}_{1}^{-1}\left(M_{Z}\right)+\Delta_{31}+\frac{\beta_{13}}{\beta_{12}}\left(\widetilde{\alpha}_{2}^{-1}\left(M_{Z}\right)-\widetilde{\alpha}_{1}^{-1}\left(M_{Z}\right)+\Delta_{12}\right),
$$

which differs from the prediction obtained by neglecting heavy thresholds by only the terms $\delta_{12}^{H}, \delta_{13}^{H}$, which reflect the heavy splitting.

In order to explicitly compare with the $\overline{D R}$ approach, the artificial decoupling 
treatment of thresholds should be employed, as described in Appendix B. This involves using a step function through each light field $l \in L$ with mass greater than $M_{Z}$, and through every superheavy field $h \in H$. Then one must impose the unification condition that the three gauge couplings are equal at the maximum mass of heavy fields, $M_{X}=\max \left\{m_{h}, h \in H\right\}$. At energies above this maximum mass, the three couplings run identically according to the beta function for the unified group $G$; hence there is no arbitrariness in the choice of the unification scale. Next, the prediction for the $\overline{D R}$ strong coupling should be translated to the $\overline{P T}$ strong coupling. Doing this, one finds the exact same form of Eq.(3.30), except that $\Delta_{i}^{L}$ is replaced by

$$
\Delta_{i}^{L} \rightarrow \sum_{m_{l}<M_{Z}} \frac{1}{4 \pi} \beta_{i}^{(l)}\left(L_{l}\left(\frac{M_{Z}}{m_{l}}\right)-\log \frac{M_{Z}^{2}}{m_{l}^{2}}\right)+\sum_{m_{l}>M_{Z}} \frac{1}{4 \pi} \beta_{i}^{(l)}\left(\eta_{l}-\log \frac{M_{Z}^{2}}{m_{l}^{2}}\right)
$$

Notice that there are only light threshold corrections beyond the theta function approximation for particles of mass above $M_{Z}$, since those below $M_{Z}$ are already implicitly accounted for. This formula is in agreement with Eqs. $[3.69,3.70]$, since there is a residual error proportional to $L_{p}\left(M_{Z} / m_{p}\right)-\eta_{p}$ for each crossed threshold. The analogous corrections for the heavy thresholds do not arise in the asymptotic unification scenario, since we are essentially comparing observables at energy scales $M_{Z} \sim m_{l}$, which is of the same order of magnitude as the light thresholds, and $M_{U} \gg m_{h}$, which is much greater than all thresholds when asymptotic unification conditions are assumed. The latter scale is a "desert" scale, and so the step function method has no errors, giving the same result obtained above in the $\delta_{i}^{H} .{ }^{8}$ For the more general unification conditions considered in subsection 3.4 .4 there will be additional heavy threshold corrections.

Eq.(3.30) is a useful result, as it allows one to constrain the heavy spectrum, given a light SUSY spectrum. Up to two-loop finite threshold corrections, which we have estimated to be small, and assuming that Eq.(3.30) will yield the experimental value of the strong coupling given some appropriate full GUT theory (i.e. assuming the

\footnotetext{
${ }^{8}$ It should be emphasized that this is only the case when the $\overline{D R}$ is correctly implemented by choosing the unification scale to be equal to the heaviest threshold in the theory. Different choices are sometimes made in the literature.
} 
asymptotic unification hypothesis is true), we can write

$$
\epsilon^{H} \equiv \widetilde{\alpha}_{3}^{-1}\left(M_{Z}\right)_{H}^{\text {pred }}-\widetilde{\alpha}_{3}^{-1}\left(M_{Z}\right)^{e x p t} \approx-\delta_{31}^{H}-\frac{\beta_{13}}{\beta_{12}} \delta_{12}^{H},
$$

where $\widetilde{\alpha}_{3}^{-1}\left(M_{Z}\right)_{H}^{\text {pred }}$ is the predicted value of the strong coupling obtained by neglecting heavy thresholds, as illustrated in Figs.[3.4,3.5]. We should emphasize the assumptions leading to this result. First, the standard normalizations of the couplings are assumed, so that Eq.(3.32) does not hold for higher affine levels or non-standard hypercharge normalizations, as often occur in string models. Second, we assume that the gluino is somewhat heavier than the chargino, so that there are serious discrepancies, as in Fig. [3.5], which must be explained by heavy threshold corrections. Finally, we are using the paradigm of asymptotic unification, wherein the full gauge group $G$ in which the SM is embedded is restored before other Planck scale physics becomes relevant. With these assumptions, and noting that heavy thresholds were neglected in Figs.[3.4,3.5], we find a typical value of

$$
\epsilon^{H} \stackrel{\operatorname{expt}}{\approx}-1 \stackrel{\text { theory }}{\approx}-\frac{1}{4 \pi} \sum_{h \in H} B_{h} \log \frac{m_{h}^{2}}{M_{X}^{2}}
$$

where we have defined ${ }^{9}$

$$
B_{h} \equiv \beta_{31}^{(h)}+\frac{12}{7} \beta_{12}^{(h)} .
$$

Values of $B^{h}$ can be compiled for the heavy representations any unified gauge group, and hence may be used with heavy mass ratios to exclude or provide evidence for a given GUT theory.

To calculate $B_{h}$, we first write $B_{h}=\bar{\beta}_{s_{h}} \bar{B}_{h}$, where $\bar{\beta}_{s_{h}}=-1 / 3,-2 / 3,11 / 3$ for spin $0,1 / 2,1$ fields and the remaining group theory factor is $\bar{B}_{h}=\frac{5}{7} T_{1}(R)-\frac{12}{7} T_{2}(R)+T_{3}(R)$ for a representation $R$. It is necessary to decompose all representations in terms of their $U(1)_{Y} \otimes S U(2)_{L} \otimes S U(3)_{C}$ content. Here $T_{1}(R)=\frac{3}{5} \sum_{p \in R} Y_{p}^{2}$ and $T_{i}(R) \delta^{a b}=$ $\sum_{p \in R} \operatorname{tr}_{i}\left(t_{R}^{a} t_{R}^{b}\right), i=2,3$. For most grand unified theories of interest, all multiplets can be decomposed in terms of only eight different standard model multiplets (plus

\footnotetext{
${ }^{9}$ Note that $\frac{\beta_{13}}{\beta_{12}}=\frac{12}{7}$.
} 


\begin{tabular}{|c|c|c|c|}
\hline & $\bar{B}_{h}\left(R_{i}\right)$ & & $\bar{B}_{h}\left(R_{i}\right)$ \\
\hline \hline$R_{1}=(\mathbf{3}, \mathbf{2}, 1 / 6)$ & $-3 / 2$ & $R_{5}=(\mathbf{1}, \mathbf{1}, 1)$ & $3 / 7$ \\
\hline$R_{2}=(\mathbf{3}, \mathbf{1},-1 / 3)$ & $9 / 14$ & $R_{6}=(\mathbf{8}, \mathbf{1}, 0)$ & 3 \\
\hline$R_{3}=(\mathbf{3}, \mathbf{1}, 2 / 3)$ & $15 / 14$ & $R_{7}=(\mathbf{1}, \mathbf{3}, 0)$ & $-24 / 7$ \\
\hline$R_{4}=(\mathbf{1}, \mathbf{2}, 1 / 2)$ & $-9 / 14$ & $R_{8}=(\mathbf{3}, \mathbf{2}, 5 / 6)$ & $3 / 14$ \\
\hline
\end{tabular}

Table 3.3: Heavy threshold constants $B_{h}$

their conjugate representations, which have the same $B_{h}$, and a singlet which has $B_{h}=0$ ), which are given in Table 3.3 along with the value of $\overline{B_{h}}$.

These same constants will also govern the corrections from analytic heavy threshold corrections that will be discussed later in section 3.4.4. Notice that, by definition, the constants satisfy the constraint that the sum over all heavy multiplets vanishes,

$$
\sum_{h \in H} B_{h}=0
$$

which equivalently reflects the arbitrariness in the choice of which heavy mass scale $M_{X}$ one chooses to be canonical (see Eq. (3.32)), $\frac{\partial \epsilon^{H}}{\partial M_{X}}=0$. A similar relation also holds for any complete representation of the grand unified group. For example, the 24 of $S U(5)$ decomposes into a singlet plus $R_{6}+R_{7}+R_{8}+\overline{R_{8}}$. From the table, we have $B_{h}\left(R_{6}\right)+B_{h}\left(R_{7}\right)+2 B_{h}\left(R_{8}\right)=0$.

As a simple example, let us explore the (unlikely) possibility wherein the only heavy field with significantly different mass than the heavy gauge boson mass $M_{X}$ is the $\mathbf{5}$ dimensional Higgs supermultiplet in which the light Higgs doublets are embedded. The triplet components of the two Higgs supermultiplets contributes $-2 / 5,0,-1$ to $\beta_{1}, \beta_{2}, \beta_{3}$, and hence $B_{h}(\mathbf{3}+\overline{\mathbf{3}})=-9 / 7$. Using Eqs. $(3.32,3.33)$, this leads to $M_{3} \approx M_{X} \exp \left(-\frac{14 \pi}{9}\right)$, which is of order $M_{X} / 100$. Such a large splitting is unnatural and difficult to accommodate in a theory. In general, "natural" splittings do not lead to $\epsilon^{H}$ values of the correct magnitude in $S U(5)$. This is not terribly surprising, since minimal SUSY $S U(5)$ is already known to be strongly disfavored.

In general, the large discrepancies in Figs.[3.4,3.5] imply a large splitting in the heavy spectrum, which, in turn may imply a multistep unification scenario, e.g. 
$S O(10) \rightarrow G_{224} \rightarrow G_{S M}$. The reason is that for the heavy fields to contribute to $\widetilde{\alpha}_{3}\left(M_{Z}\right)$, they must not only have a mass splitting compared to some reference heavy gauge boson, $X$, but also must have different first beta function coefficients since only the differences $\beta_{12}^{(h)} \equiv \beta_{1}^{(h)}-\beta_{2}^{(h)}$ and $\beta_{31}^{(h)} \equiv \beta_{3}^{(h)}-\beta_{1}^{(h)}$ appear in the corrections.

Before moving to more general unification boundary conditions, we shall give a simple way to define an effective unification scale in the asymptotic unification scenario.

\subsubsection{Effective Unification Scale}

Because the couplings formally unify at infinite energy in the paradigm of asymptotic unification, there is no apparent unification scale. However, we suspect that in reality quantum gravitational fluctuations will affect the couplings as they approach the Planck energy. Hence, one can define an effective unification scale to be where the splittings between the gauge couplings are of the same order as those induced by gravitational effects. To be precise, define a dimensionless gravitational coupling which classically runs with energy as

$$
G(Q)=\frac{Q^{2}}{M_{P l}^{2}},
$$

where $M_{P l} \approx 1.22 \times 10^{19} \mathrm{GeV}$. The leading gravitational corrections to the running gauge couplings $\alpha_{i}(Q)$ will be proportional to $G(Q) \alpha_{i}(Q)$. Hence, the effective asymptotic unification scale, $M_{e f f}$, can be defined as the scale where the splittings in the gauge couplings are of order the gravitational corrections, $\mid \alpha_{i}\left(M_{e f f}\right)-$ $\alpha_{j}\left(M_{e f f}\right) \mid \approx b^{2} G\left(M_{e f f}\right) \alpha_{U}$, or equivalently

$$
\left|\alpha_{i}^{-1}\left(M_{e f f}\right)-\alpha_{j}^{-1}\left(M_{e f f}\right)\right| \approx b^{2} G\left(M_{e f f}\right) \alpha_{U}^{-1} \equiv \delta_{g}\left(M_{e f f}\right),
$$

where we take $\alpha_{U}^{-1} \sim 24$ to be the typical gauge coupling near unification. The unknown parameter $b^{2}$ should be of order one. Estimating $M_{\text {eff }}$ using a simple $S U(5)$ model, we find a typical effective unification scale of $1-5 \times 10^{17} \mathrm{GeV}$. This is only intended to a very rough approximation since a naively simple $S U(5)$ model was used. 
Nevertheless, more complicated and realistic GUT models yield a unification scale in the same ballpark. It is generally true that our effective unification scale is about an order of magnitude or more greater than what is typically called the unification scale $\left(\sim 2 \times 10^{16} \mathrm{GeV}\right)$.

It may seem that our definition of an effective unification scale is rather artificial. However, it may be physically motivated by the following considerations. If indeed the standard model is embedded in some unified theory of gravity and gauge forces, then there may exist a phase at energies below the Planck scale which consists of a simple Lie group containing the supersymmetric standard model. In the absence of any gravitational corrections, the running gauge couplings certainly unify asymptotically, as this is the only case in which the higher group symmetry is fully realized up to arbitrarily high energies. Hence, the running couplings should only deviate from asymptotic unification by the gravitational corrections parameterized above. So, by the above reasoning, the effective unification scale should roughly correspond to the physical unification scale when the full (quantum gravitational) theory is considered.

These results may have consequences for the paradigm of string unification. In particular, one problem of string unification [43] is that the couplings seem to unify at a scale $\left(M_{G}^{\overline{D R}} \approx 2 \times 10^{16} \mathrm{GeV}\right)$ about twenty times lower than the scale predicted by four dimensional heterotic string models $\left(M_{G}^{\text {string }} \approx 5 \times 10^{17} \mathrm{GeV}\right)$. In the approach presented here, heavy threshold effects seem to push the effective asymptotic unification scale to roughly $M_{G}^{\text {string }}$. Despite the apparent success, this coincidence cannot be taken seriously until several questions are addressed in regards to this so-called string gauge coupling problem. First, the calculation of $M_{G}^{\text {string }}$ [44] was performed in the $\overline{D R}$ scheme, with the field theory step-function treatment generalized to strings. An analogous calculation for physical renormalization schemes is lacking, so it is difficult to compare our results with string predictions. Secondly, the asymptotic unification boundary conditions are probably not valid for many string models, and so the unification scale will be further changed by more general boundary conditions, as discussed in the next section. See [45] for a recent string calculation of threshold corrections to grand unification. 


\subsubsection{More General Boundary Conditions}

The discussion of this section concerns the next-simplest boundary conditions after asymptotic unification. In particular, we will impose $\alpha_{1}\left(M_{U}\right)=\alpha_{2}\left(M_{U}\right)=\alpha_{3}\left(M_{U}\right)$ at scale $M_{U} \sim M_{h}$, for some $h \in H$. As discussed in the previous section, one might expect $M_{U}$ to roughly correspond to the asymptotic unification scale, which we found to be roughly $5 \times 10^{17} \mathrm{GeV}$. However, we will consider $M_{U}$ as an input and find the corrections for the strong coupling and gauge boson mass predictions.

Before proceeding, there is a subtle point that should be addressed. Notice that in the previous section, we assumed that unification would have occurred asymptotically were it not for gravitational corrections. Hence, starting with a finite unification scale and then neglecting gravitational corrections, as we do in this section, does not seem logically consistent with what was done in the previous section. This observation is entirely correct, but the point is that indeed we are considering two orthogonal scenarios, one where a finite unification scale is obtained from gravity, and another where finite unification scale is obtained from non-trivial threshold corrections. The latter case may have its origin in stringy or gravitational physics, but nevertheless becomes manifest through purely field theoretic mechanisms.

The corrections from imposing finite unification scale are straightforward to derive and can be stated in terms of the $\Delta_{i}=\Delta_{i}^{L}+\delta_{i}^{H}+\theta_{i}$ which we defined earlier. This gains an additional contribution and can now be written

$$
\Delta_{i}=\Delta_{i}^{L}+\delta_{i}^{H}+\theta_{i}-\Delta_{i}^{H}
$$

where

$$
\Delta_{i}^{H}=\sum_{h \in H} \frac{1}{4 \pi} \beta_{i}^{(h)}\left(L_{h}\left(\frac{M_{U}}{m_{h}}\right)-\log \frac{M_{U}^{2}}{m_{h}^{2}}\right),
$$

which is of exactly the same form expected from Eq. $(3.69)^{10}$. Evidently, these are finite heavy threshold corrections in addition to the corrections from the heavy threshold splittings. Hence, the $\epsilon^{H}$ defined earlier will get an additional contribution from

\footnotetext{
${ }^{10}$ This is not obvious; one must work through the derivation to see that indeed the expected $L_{p}-\eta_{p}$ correction terms do arise.
} 
the $\Delta_{i}^{H}$ 's and is now

$$
\begin{aligned}
\epsilon^{H} \stackrel{\text { theory }}{\approx} \frac{1}{4 \pi} \sum_{h \in H} B_{h}\left[\log \frac{m_{h}^{2}}{M_{X}^{2}}-\left(L_{h}\left(\frac{M_{U}}{m_{h}}\right)-\log \frac{M_{U}^{2}}{m_{h}^{2}}\right)\right] \\
\quad=-\frac{1}{4 \pi} \sum_{h \in H} B_{h} L_{h}\left(\frac{M_{U}}{m_{h}}\right) .
\end{aligned}
$$

Experimentally, $\epsilon^{H} \approx-1$, as seen in Figs.[3.4,3.5] for typical gluino to wino mass ratios; this value can be easily adjusted for nonstandard sparticle spectra. This is our final formula which may be used to assess the experimental validity of gauge coupling unification in any specific GUT model where the gauge group, superheavy mass ratios, and light SUSY masses are given.

Let us now consider the numerical size of these new threshold corrections. From Eq.(3.6) one finds that

$$
L_{h}\left(\frac{M_{U}}{m_{h}}\right)-\log \frac{M_{U}^{2}}{m_{h}^{2}} \approx \log \left(1+\frac{m_{h}^{2}}{M_{U}^{2}} e^{\eta_{h}}\right)
$$

which can be larger than heavy splitting corrections $\log \frac{m_{h}^{2}}{M_{X}^{2}}$ for values of $M_{U}$ that are not too large. Hence, such corrections cannot be neglected.

The value of $M_{U}$ is not fixed a priori, and corresponds to the physically meaningful energy where the couplings become equal due to the new nontrivial heavy threshold corrections. This complicates the analysis of unification by introducing another parameter beyond those that are usually needed. However, this is to be expected, since a new physical phenomena (corrections arising from the virtuality of very massive particles) has been included.

\subsection{Conclusions}

We have developed a new way of looking at detailed predictions of gauge coupling unification which is more physically motivated than conventional approaches. In addition to a dramatic paradigmatic improvement, novel heavy and light threshold 
corrections are obtained, and the resulting corrections to unification predictions are presented for a general GUT model. A natural extension of this work is a thorough analysis and classification of various unified theories. By calculating the $B_{h}$ constants and the heavy spectrum, one may exclude or verify the gauge unification of a given model.

Acknowledgements We wish to thank Ratin Akhoury and Eduardo de Rafael for reading a draft of this chapter and providing useful suggestions.

\subsection{Appendix A : Pinch Technique Couplings for the MSSM}

Here we will give explicit formulae for the pinch technique effective couplings regularized using dimensional reduction (DRED), which will be denoted with a tilde. These effective charges will be similar to those constructed in [30][32] for QCD, and in [31][33] for the electroweak sector. However, we will extend these to the minimal supersymmetric case, which involves explicitly including another Higgs doublet, and regulating the loop integrals with dimensional reduction (DRED), as opposed to dimensional regularization (DREG), which is used in most non-supersymmetric settings. It is well known that DRED preserves both supersymmetry and gauge symmetry. Also, the effective charges presented in [33] were in the on-shell subtraction scheme $\left(Q_{0}=0\right)$, whereas here we will need the result for arbitrary renormalization scale. In the appropriate limits our results reduce to those given in [32] and [33].

The charges are constructed using the pinch-technique (PT), which allows one to extract the universal self-energy function in non-abelian gauge theories, thus leading to gauge invariant effective couplings which

- contain explicit and complete mass-threshold behavior and

- reproduce the conventional massless beta function in the limit where masses can be neglected. 
At one-loop, the spin $1 / 2$ and spin 0 contributions to the PT gauge-boson selfenergies are trivially just the usual transverse vacuum polarization graphs. Only the graph with a gauge boson loop needs to have the self-energy-like part projected, as described briefly in section 3.3, and in more detail in the references [32][33]. In calculating the following, we used both the direct diagrammatic pinch technique algorithm [32] and the dispersive derivation from physical cross sections [33].

The PT effective charges naturally measure the self-energy-like propagation of a gauge boson and hence can be interpreted as measuring the real force between two fermions of arbitrary mass, analogous to the QED effective charge. The PT charge includes finite mass recoil effects that are missed in the heavy quark effective charge (the V-scheme). In fact, one may obtain the heavy quark potential in the appropriate kinematical limit of the pinch technique effective charge [32]. The difference between the two are due to finite mass test-charge effects that are not present in the (V) charge but are in the (PT) charge. The extension of the PT effective charge beyond one-loop has been put forth in Refs. [35, 36, 37, 38, 39].

\subsubsection{QCD Effective Charges}

The $\overline{P T}$ self-energy function for supersymmetric QCD, $\widetilde{\Pi}_{3}$, can be used to define the effective coupling for supersymmetric QCD by

$$
\widetilde{\alpha}_{3}(Q)=\frac{\widetilde{\alpha}_{3}\left(Q_{0}\right)}{1+\widetilde{\Pi}_{3}\left(Q, Q_{0}\right)} .
$$


The function $\widetilde{\Pi}_{3}$ can be written down straightforwardly using Eqs. $[3.5,3.8]$, and the unsubtracted result is given by

$$
\begin{aligned}
\widetilde{\Pi}_{3}(Q) & =\frac{\widetilde{\alpha}_{3}\left(Q_{0}\right)}{4 \pi}\left[\frac{11}{3} N_{c}\left(\log \frac{Q^{2}}{\mu^{2}}-C_{U V}-64 / 33\right)\right. \\
& -\frac{2}{3} N_{c}\left(L_{\frac{1}{2}}\left(Q / m_{\tilde{g}}\right)+\log \left(m_{\tilde{g}}^{2} / \mu^{2}\right)-C_{U V}-5 / 3\right) \\
& -\sum_{q} \frac{2}{3}\left(L_{\frac{1}{2}}\left(Q / m_{q}\right)+\log \left(m_{q}^{2} / \mu^{2}\right)-C_{U V}-5 / 3\right) \\
& \left.-\sum_{\tilde{q}} \frac{1}{3}\left(L_{0}\left(Q / m_{\tilde{q}}\right)+\log \left(m_{\tilde{q}}^{2} / \mu^{2}\right)-C_{U V}-8 / 3\right)\right] .
\end{aligned}
$$

The four terms correspond respectively to the gluons, gluinos $(\widetilde{g})$, Dirac quarks $(q)$, and to complex squark doublets $(\widetilde{q})$. For the scalars we will take the left and right components to be degenerate in mass since such complications do not change the unification predictions to any numerical significance. In any case, one may trivially treat the two separately.

To relate the resulting effective charge to other schemes or observables one needs to use Eq.(3.10).

Eq.(3.43) can be written in a more useful once subtracted form by relating the effective charges at different scales, leading to an expression governing the running of the charge given by

$$
\begin{aligned}
\widetilde{\Pi}_{3}\left(Q, Q_{0}\right) & \equiv \widetilde{\Pi}_{3}(Q)-\widetilde{\Pi}_{3}\left(Q_{0}\right) \\
& =\frac{\widetilde{\alpha}_{3}\left(Q_{0}\right)}{4 \pi}\left[\frac{11}{3} N_{c}\left(\log \frac{Q^{2}}{Q_{0}^{2}}\right)-\frac{2}{3} N_{c}\left(L_{\frac{1}{2}}\left(Q / m_{\tilde{g}}\right)-L_{\frac{1}{2}}\left(Q_{0} / m_{\tilde{g}}\right)\right)\right. \\
& -\sum_{q} \frac{2}{3}\left(L_{\frac{1}{2}}\left(Q / m_{q}\right)-L_{\frac{1}{2}}\left(Q_{0} / m_{q}\right)\right) \\
& \left.-\sum_{\tilde{q}} \frac{1}{3}\left(L_{0}\left(Q / m_{\tilde{q}}\right)-L_{0}\left(Q_{0} / m_{\tilde{q}}\right)\right)\right] .
\end{aligned}
$$

Though the gluon contribution in Eq.(3.43) looks simple, it is actually the most 
difficult piece to compute. As discussed in [32], the pinch technique self-energy that is used to define the effective charge is gauge and scale independent, and indeed reproduces the pure gauge term of the $\beta$ function coefficient $\left(11 N_{c} / 3\right)$. This of course is not the case for the full pure gluon vacuum polarization, which is gauge and scale dependent and does not reproduce the correct $\beta$ function. The non-trivial and important part of Eq.(3.43) is the constant 64/33, which specifies the scheme. This constant may be obtained by calculating the pinch-technique gluon self-energy using DRED. To translate to DREG one just subtracts 1/11 (from the so-called epsilon ghosts) to get the constant $67 / 33$.

For comparison, the heavy quark potential effective charge, $\alpha_{V}$, has the values 28/33 and 31/33 when using DRED and DREG, respectively. Consequently, the Vscheme doesn't satisfy the decoupling criterion of Eq.(3.11). This is just a reflection of the fact that infinitely heavy external quarks are used in the $\mathrm{V}$-scheme calculation, thus rendering meaningless the limit where internal virtual particles acquire very large mass.

Notice that in the appropriate limit the above reduces to the standard RG $\beta$ function coefficient for supersymmetric QCD,

$$
\lim _{m_{\imath} \rightarrow 0} \widetilde{\Pi}_{3}=\frac{\alpha_{s}}{4 \pi}\left(9-n_{f}\right) \log \frac{Q^{2}}{Q_{0}} .
$$

\subsubsection{The Electroweak Sector}

The effective QED charge and the effective weak-mixing angle are obtained by diagonalizing the electroweak neutral currents and are given by [33]

$$
\widetilde{\alpha}(Q)=\frac{\widetilde{\alpha}\left(Q_{0}\right)}{1+\widetilde{\Pi}_{\gamma \gamma}\left(Q, Q_{0}\right)},
$$

and

$$
\widetilde{s}_{w}^{2}(Q)=\widetilde{s}_{w}^{2}\left(Q_{0}\right)\left(1+\frac{\widetilde{c}_{w}\left(Q_{0}\right)}{\widetilde{s}_{w}\left(Q_{0}\right)} \frac{\widetilde{\Pi}_{\gamma Z}\left(Q, Q_{0}\right)}{1+\widetilde{\Pi}_{\gamma \gamma}\left(Q, Q_{0}\right)}\right)
$$


For the matter sector, we will write only the subtracted $\overline{P T}$ self-energies, as it is now clear how to translate between schemes using the $\eta_{p}$ constants as described before. The quarks $(q)$ and leptons $(l)$, along with their scalar superpartners $(\widetilde{q}, \widetilde{l})$, yield

$$
\begin{aligned}
\widetilde{\Pi}_{\gamma \gamma}(\text { matter }) & =\frac{\widetilde{\alpha}\left(Q_{0}\right)}{4 \pi}\left[-\sum_{q} \frac{4}{3} N_{c} e_{q}^{2}\left(L_{\frac{1}{2}}\left(Q / m_{q}\right)-L_{\frac{1}{2}}\left(Q_{0} / m_{q}\right)\right)\right. \\
& -\sum_{\tilde{q}} \frac{2}{3} N_{c} e_{\tilde{q}}^{2}\left(L_{0}\left(Q / m_{\tilde{q}}\right)-L_{0}\left(Q_{0} / m_{\tilde{q}}\right)\right) \\
& -\sum_{l} \frac{4}{3}\left(L_{\frac{1}{2}}\left(Q / m_{l}\right)-L_{\frac{1}{2}}\left(Q_{0} / m_{l}\right)\right) \\
& \left.-\sum_{\tilde{l}} \frac{2}{3}\left(L_{0}\left(Q / m_{\tilde{l}}\right)-L_{0}\left(Q_{0} / m_{\tilde{l}}\right)\right)\right]
\end{aligned}
$$

The electric charge of a particle $p$ is denoted $e_{p}$. The analogous contribution of individual Dirac mass eigenstate matter fields to the $\gamma Z$ self energy are given by the relation

$$
\widetilde{\Pi}_{\gamma Z}^{(p)}=\left(\frac{1}{4\left|e_{p}\right|}-\widetilde{s}_{w}^{2}\right) \frac{1}{\widetilde{s}_{w} \widetilde{c}_{w}} \widetilde{\Pi}_{\gamma \gamma}^{(p)},
$$

where $p$ denotes any of the fermions or scalars above.

The contribution of the charged vector bosons to the self-energies is more complicated than the matter multiplets. Similar to the QCD case, the non-abelian nature of the theory implies that $W^{+} W^{-}$loops (along with possible gauge dependent ghost and Goldstone boson loops), do not yield a gauge invariant result, and do not give the appropriate contribution to the electroweak beta functions. The proper treatment involves calculating the self-energy like part of the one-loop $e^{+} e^{-} \rightarrow e^{+} e^{-}$amplitude (or using any other fermions due to universality), including vertex and box corrections involving neutrinos. These contribute pinched parts which make the self-energy-like part gauge invariant and transverse. This calculation was first performed in [31], and then with dispersion relations in [33], for $n_{H}=1$ Higgs doublets and renormalized in the on-shell scheme at $Q_{0}=0$. Here we need to extend these results to arbitrary $n_{H}$ and $Q_{0}$, and would like to have the finite constants in the unrenormalized expression, 
including constant terms arising from using DRED instead of DREG. The most efficient way to do this is to use the Feynman gauge $\xi=1$, where $W^{ \pm}$bosons, $-G^{ \pm}$ Goldstone bosons, and $\eta^{ \pm}$ghosts all propagate with $-i g_{\mu \nu} /\left(p^{2}-M_{W}^{2}\right)$. Hence, the only factors of transverse momenta arise from the three boson vertex, and so the box graph and several of the vertex graphs may be neglected, as they do not have pinched parts. Here, the dependence on the Higgs doublets comes only from the unphysical charged Goldstone scalars for the $n_{H}=1$ case, and also on charged Higgs for $n_{H}>1$. The result for the $S U(2)_{L} \otimes U(1)_{Y}$ electroweak theory is

$$
\begin{aligned}
\widetilde{\Pi}_{\gamma \gamma}^{(W, H)} & =\frac{\widetilde{\alpha}\left(Q_{0}\right)}{4 \pi}\left[2 \frac{11}{3}\left(\overline{L_{1}}\left(Q / M_{W}\right)+\log \left(M_{W}^{2} / \mu^{2}\right)-C_{U V}-64 / 33\right)\right. \\
& \left.+\sum_{a=1}^{n_{H}}\left(-\frac{1}{3}\right)\left(L_{0}\left(Q / M_{a}\right)+\log \left(M_{a}^{2} / \mu^{2}\right)-C_{U V}-8 / 3\right)\right]
\end{aligned}
$$

where the constant $64 / 33$ is the same as appeared for the gluon self energy. The sum in the second line will be over mass eigenstate charged Higgs scalars; there will be one of these for each Higgs doublet in the theory. The first scalar ( $a=1$ in the sum) is an unphysical Goldstone boson that is eaten by the $W^{ \pm}$, and hence one identifies its mass to be $M_{1}=M_{W}$ (in the Feynman gauge). The second charged scalar $(\mathrm{a}=2)$ is conventionally denoted by $H^{ \pm}$in the MSSM, with mass $M_{2}=M_{H^{ \pm}}$. Additional Higgs doublets beyond the MSSM are not considered here so we can take $n_{H}=2$. The function

$$
\overline{L_{1}}(Q / m)=\frac{2}{11}\left(\beta \tanh ^{-1} \beta^{-1}\left(12-\beta^{2}\right)+\beta^{2}-1\right)
$$

with $\beta=\sqrt{1+\frac{4 m^{2}}{Q^{2}}}$, comes from the $W^{+} W^{-}$and ghost loops, the $W^{+} G^{-}+W^{-} G^{+}$ loops, and the pinched self-energy-like part of the $\gamma W W$ vertex where the internal neutrino line is pinched. The $L_{0}$ comes from the charged Goldstones and Higgs loops. As might be anticipated from the fermions and scalars, where, for example, $\lim _{m \rightarrow \infty} L_{\frac{1}{2}}(Q / m)=5 / 3$ is the same constant as appears in the self-energy, we also have the nice property that

$$
\lim _{M_{W} \rightarrow \infty} \overline{L_{1}}\left(Q / M_{W}\right)=64 / 33
$$


Letting the W-bosons eat the Goldstones by performing simple algebra in Eq.(3.50), one finds the result written in terms of physical degrees of freedom,

$$
\begin{aligned}
\widetilde{\Pi}_{\gamma \gamma}^{(W, H)} & =\frac{\widetilde{\alpha}\left(Q_{0}\right)}{4 \pi}\left[7\left(L_{1}\left(Q / M_{W}\right)+\log \left(M_{W}^{2} / \mu^{2}\right)-C_{U V}-40 / 21\right)\right. \\
& \left.+\sum_{a=2}^{n_{H}}\left(-\frac{1}{3}\right)\left(L_{0}\left(Q / M_{a}\right)+\log \left(M_{a}^{2} / \mu^{2}\right)-C_{U V}-8 / 3\right)\right]
\end{aligned}
$$

The contribution of the physical massive gauge boson is characterized by $7 L_{1}=$ $(22 / 3) \overline{L_{1}}+(-1 / 3) L_{s}$, explicitly given by

$$
L_{1}(Q / m)=2 \beta \tanh ^{-1} \beta^{-1}\left(1-\left(\beta^{2}-1\right) / 7\right)+(2 / 7)\left(\beta^{2}-1\right)
$$

As expected,

$$
\lim _{m \rightarrow 0} L_{W}(Q / m)=\log \frac{Q^{2}}{m^{2}} \quad \lim _{m \rightarrow \infty} L_{W}(Q / m)=40 / 21
$$

Notice that Eq.(3.54) precisely corresponds to Eq.(3.19) for $s=1$.

The separation of pure gauge effects and those arising in the broken phase of the theory is useful, and allows us to immediately write down the analogous result for $\gamma Z$ without further calculation :

$$
\begin{aligned}
\widetilde{\Pi}_{\gamma Z}^{(W, H)} & =\frac{\widetilde{\alpha}\left(Q_{0}\right)}{4 \pi c_{w} s_{w}}\left[2 \frac{11}{3} c_{w}^{2}\left(\overline{\overline{L_{1}}}\left(Q / M_{W}\right)+\log \left(M_{W}^{2} / \mu^{2}\right)-C_{U V}-64 / 33\right)\right. \\
& \left.+\sum_{a=1}^{n_{H}}\left(-\frac{1}{3}\left(c_{w}^{2}-\frac{1}{2}\right)\right)\left(L_{0}\left(Q / M_{a}\right)+\log \left(M_{a}^{2} / \mu^{2}\right)-C_{U V}-8 / 3\right)\right]
\end{aligned}
$$

Finally, the wino and charged Higgsino, whose mixing is neglected, contribute

$$
\widetilde{\Pi}_{\gamma \gamma}^{\widetilde{H}, \widetilde{W}}=\frac{\widetilde{\alpha}\left(Q_{0}\right)}{4 \pi}\left[-\frac{4}{3}\left(L_{\frac{1}{2}}\left(\frac{Q}{m_{\widetilde{H}}}\right)-L_{\frac{1}{2}}\left(\frac{Q_{0}}{m_{\widetilde{H}}}\right)\right)-\frac{4}{3}\left(L_{\frac{1}{2}}\left(\frac{Q}{m_{\widetilde{W}}}\right)-L_{\frac{1}{2}}\left(\frac{Q_{0}}{m_{\widetilde{W}}}\right)\right)\right]
$$


and

$$
\widetilde{\Pi}_{\gamma Z}^{\widetilde{H} \widetilde{W}}=\frac{\widetilde{\alpha}\left(Q_{0}\right)}{4 \pi c_{w} s_{w}}\left[-\frac{4}{3} c_{w}^{2}\left(L_{\frac{1}{2}}\left(\frac{Q}{m_{\tilde{H}}}\right)-L_{\frac{1}{2}}\left(\frac{Q_{0}}{m_{\widetilde{H}}}\right)\right)-\frac{4}{3}\left(c_{w}^{2}-1 / 2\right)\left(L_{\frac{1}{2}}\left(\frac{Q}{m_{\widetilde{W}}}\right)-L_{\frac{1}{2}}\left(\frac{Q_{0}}{m_{\widetilde{W}}}\right)\right)\right]
$$

The $S U(2)_{L} \otimes U(1)_{Y}$ effective couplings constructed from the above results are

$$
\widetilde{\alpha_{i}}(Q)=\frac{\widetilde{\alpha}_{i}\left(Q_{0}\right)}{1+\widetilde{\Pi}_{i}\left(Q, Q_{0}\right)},
$$

for $i=1,2$ and $\widetilde{\alpha_{i}}(Q)$ given in Eq.(3.18). The PT self-energies are related by

$$
\begin{aligned}
& \widetilde{\Pi}_{1}=\widetilde{\Pi}_{\gamma \gamma}-\frac{s_{w}}{c_{w}} \widetilde{\Pi}_{\gamma Z} \\
& \widetilde{\Pi}_{2}=\widetilde{\Pi}_{\gamma \gamma}+\frac{c_{w}}{s_{w}} \widetilde{\Pi}_{\gamma Z} .
\end{aligned}
$$

Notice that $\widetilde{\Pi}_{\gamma \gamma}=\sum_{p} \widetilde{\Pi}_{\gamma \gamma}^{(p)}$ and $\widetilde{\Pi}_{\gamma Z}=\sum_{p} \widetilde{\Pi}_{\gamma Z}^{(p)}$ (as well as $\widetilde{\Pi}_{i}$ ) have the correct beta function coefficients, which are summarized below, and smoothly interpolate between all mass thresholds. The full mass-dependent beta functions may be obtained by differentiating the above expressions, but we will just give the massless limits, in order to make clear our conventions.

The one-loop beta function coefficients are defined by the relations

$$
\begin{gathered}
\frac{d \alpha}{d \log Q^{2}}=-\alpha \frac{d \widetilde{\Pi}_{\gamma \gamma}}{d \log Q^{2}}=-\frac{\alpha^{2}}{4 \pi} \beta_{\gamma \gamma} \\
\frac{d s_{w}^{2}}{d \log Q^{2}}=s_{w} c_{w} \frac{d \widetilde{\Pi}_{\gamma Z}}{d \log Q^{2}}=\frac{\alpha}{4 \pi} \beta_{\gamma Z} \\
\beta_{1}=\frac{3}{5}\left(c_{w}^{2} \beta_{\gamma \gamma}-\beta_{\gamma Z}\right) \\
\beta_{2}=s_{w}^{2} \beta_{\gamma \gamma}+\beta_{\gamma Z} .
\end{gathered}
$$


One finds

$$
\begin{aligned}
& \beta_{\gamma \gamma}=-\frac{16}{3} N_{g}+6-n_{H} \\
& \beta_{\gamma Z}=-2 N_{g}+\frac{16}{3} s_{w}^{2} N_{g}+6 c_{w}^{2}-n_{H}\left(\frac{1}{2}-s_{w}^{2}\right),
\end{aligned}
$$

which lead to the correct MSSM coefficients given below in Eq.(4.16).

\subsection{Appendix B: Unphysical Schemes and Decou- pling}

A more detailed treatment of thresholds and decoupling will now be discussed. The intrinsic difficulties with unphysical schemes such as $\overline{M S}$ will be highlighted.

Notice that the physical renormalization scheme requirement Eq.(3.11) is not met by $\overline{M S}, \overline{D R}$, their massive extensions, or similar schemes. It is well known that $\overline{M S}$ by itself does not constitute a complete scheme, rather one must truncate the sum over $p$ to include only particles with masses less than the scale of the problem. For each region between thresholds a different scheme is implemented and one must translate between schemes when crossing thresholds. Hence, $\overline{M S}$ is really a set of schemes related to each other. We will call such a set an artificial decoupling scheme (ADS), and now discuss the most general case at one loop. This will give us an idea of the discrepancies one may expect in ADS's when compared to the physical renormalization scheme approach.

Let $S=\left\{m_{1}, m_{2}, \ldots\right\}$ be the spectrum of massive particles of the fundamental theory ordered from lightest to heaviest, let $S_{0}$ be the set of massless particles, and let $S^{N}=S_{0} \ominus\left\{m_{1}, m_{2}, \ldots, m_{N}\right\}$ be some subset up to a given mass scale. For any given renormalization scheme $R$, let the $N$ th phase of $R$, denoted $R^{N}$, be the scheme used to renormalize observables at energy scales $Q$ such that

$$
m_{1}, \ldots, m_{N}<Q<m_{N+1} .
$$

To use $R^{N}$, one simply renormalizes the contributions from particles $p \in S^{N}$ in the 
usual way dictated by scheme $R$, and then entirely neglects the contributions from all $p \in S-S^{N}$. This is a formal statement of the usual implementation of ADS's using step functions.

For $m_{1}, \ldots, m_{N}<Q, Q^{\prime}<m_{N+1}$, the gauge coupling of the $R^{N}$ scheme flows by

$$
\begin{aligned}
\alpha_{R^{N}}(Q) & =\alpha_{R^{N}}\left(Q^{\prime}\right)-\frac{\left(\alpha_{R^{N}}\left(Q^{\prime}\right)\right)^{2}}{4 \pi} \sum_{p \in S^{N}} \beta_{p}\left(L_{p}\left(Q / m_{p}\right)-L_{p}\left(Q^{\prime} / m_{p}\right)\right. \\
& \left.-\eta_{p}^{R}\left(Q / m_{p}\right)+\eta_{p}^{R}\left(Q^{\prime} / m_{p}\right)\right)
\end{aligned}
$$

and the most general matching condition between schemes $R^{N-1}$ and $R^{N}$ takes the form

$$
\alpha_{R^{N-1}}\left(\mu_{N}\right)=\alpha_{R^{N}}\left(\mu_{N}\right)+\frac{\alpha_{R^{N}}^{2}\left(\mu_{N}\right)}{4 \pi} \beta_{N} A_{N}\left(\mu_{N} / m_{N}\right),
$$

where $A_{p}\left(\mu_{p} / m_{p}\right)$ is arbitrary now, but will be specified below by minimizing errors, and may depend only on the matching scale $\mu_{p}$ for each threshold $m_{p}$, for reasons discussed below. The first $\beta$ function coefficient of particle $N, \beta_{N}$, is pulled out by convention.

For $Q_{h}>m_{N+n}$ and $m_{1}, \ldots, m_{N}<Q_{l}<m_{N+1}$ (the $h$ and $l$ stand for heavy and light scales, respectively) one may relate observables by flowing through $n$ thresholds using the above formulas to obtain

$$
\begin{aligned}
\alpha^{\mathcal{O}_{1}}\left(Q_{h}\right) & =\alpha^{\mathcal{O}_{2}}\left(Q_{l}\right)-\frac{\left(\alpha^{\mathcal{O}_{2}}\left(Q_{l}\right)\right)^{2}}{4 \pi}\left[\sum _ { p \in S ^ { N + n } } \beta _ { p } \left(L_{p}\left(Q_{h} / m_{p}\right)-L_{p}\left(Q_{l} / m_{p}\right)\right.\right. \\
& \left.-\eta_{p}^{\mathcal{O}_{1}}\left(Q_{h} / m_{p}\right)+\eta_{p}^{\mathcal{O}_{2}}\left(Q_{l} / m_{p}\right)\right)+\sum_{p \in S^{N+n}-S^{N}} \beta_{p}\left(A_{p}\left(\mu_{p} / m_{p}\right)-L_{p}\left(\mu_{p} / m_{p}\right)\right. \\
& \left.\left.+\eta_{p}^{R}\left(\mu_{p} / m_{p}\right)+L_{p}\left(Q_{l} / m_{p}\right)-\eta_{p}^{\mathcal{O}_{2}}\left(Q_{l} / m_{p}\right)\right)\right] .
\end{aligned}
$$

Now let us compare this to the relation, Eq.(3.10), obtained in the previous section for the tracking of two observables. For the case of a high scale desert region $\left(Q_{h} \ll m_{N+n+1}\right)$, the first sum reduces to Eq.(3.10). However, when $Q_{h} \lesssim m_{N+n+1}$, 
there are errors of one loop order which are proportional to $L_{p}\left(Q_{h} / m_{p}\right)-\eta_{p}^{\mathcal{O}_{1}}\left(Q_{h} / m_{p}\right)$, and occur for each neglected threshold ${ }^{11} p \in S-S^{N+n}$ such that $Q_{h} \lesssim m_{p}$. These errors are naturally remedied in physical renormalization schemes, leading to heavy threshold corrections which will be of importance when grand unification is discussed later. There are also analogous light threshold corrections. The second sum in Eq.(3.69) contains extra terms which arise from the artificial decoupling and matching conditions at each threshold. These terms are also generally of order of the one-loop corrections and must cancel if the ADS is to be consistent (in the sense of giving reliable physical predictions in relations between two observables). In general, these terms do not cancel, since the $Q_{l}$-dependent terms cannot be canceled by the choice of $A_{p}$, which depends only on the ratio $\mu_{p} / m_{p}$. Suitably choosing $A_{p}$ (see Eq.3.70 below) leaves a term $L_{p}\left(Q_{l} / m_{p}\right)-\eta_{p}^{\mathcal{O}_{2}}\left(Q_{l} / m_{p}\right)$ for all $p \in S^{N+n}-S^{N}$. Hence, we see that the high scale and low scale threshold corrections have exactly the same form; indeed, they have the same origin, namely the necessity of using an ADS, which arises from improper decoupling. .

In the case of a low scale desert region, $Q_{l} \ll m_{p} \forall p \in S^{N+n}-S^{N}$, one finds that $L_{p}\left(Q_{l} / m_{p}\right)-\eta_{p}^{\mathcal{O}_{2}}\left(Q_{l} / m_{p}\right) \rightarrow 0$ by Eqs. $(3.7,3.11)$, and the light threshold errors are eliminated through the choice

$$
A_{p}\left(\mu_{p} / m_{p}\right)=L_{p}\left(\mu_{p} / m_{p}\right)-\eta_{p}^{R}\left(\mu_{p} / m_{p}\right) .
$$

In this case, notice that once the $A_{p}$ are chosen suitably, the matching scale $\mu_{p}$ exactly cancels and there is no need to fix its value. However, for $\overline{M S}$ we have $A_{p}=\log \left(\mu_{p}^{2} / m_{p}^{2}\right)$ (see the discussion below Eq.(3.9)) and so this choice is equivalent to using $A_{p}=0$ and $\mu_{p}=m_{p}$, which is the matching scale typically used at one loop. One may object that even in non-desert regions the known anomalous matching threshold errors could be systematically subtracted off for each physical process considered (equivalently allowing $A_{p}=A_{p}\left(Q_{l}\right)$ ). This is awkward, as it is the same as using a different coupling for each process, thus losing the remnants of universality left by ADS's (i.e. universality in each desert region).

\footnotetext{
${ }^{11}$ similar errors occur if $Q_{l} \lesssim m_{N+n+1}$
} 
We have identified two potential problems in artificial decoupling schemes, which arise solely from the failure of the decoupling requirement given in Eq.(3.11), regardless of whether or not the scheme $R$ has analytic threshold dependence. ${ }^{12}$ The low scale errors come from the matching conditions and are exhibited in the last two terms of Eq.(3.69). These are significant only when $Q_{l} \lesssim m_{N+1}$. The high scale errors occur when one is calculating an observable at an energy $Q_{h} \lesssim m_{N+n+1}$ that is slightly less than masses that should contribute, but are cut off in an ADS. These two errors will give rise to light and heavy threshold corrections in unification, as discussed in section IV.

In practice, both types of errors can often be eliminated through a "threshold shifting" procedure. This involves modifying the definition of $R^{N}$ by replacing Eq.(3.66) with $m_{1}, \ldots, m_{N}<a Q<m_{N+1}$ and making similar subsequent replacements, and by choosing $a>1$ to be large enough so that the desired thresholds that are slightly above $Q_{l}$ or $Q_{h}$ are 'moved' below $a Q_{l}$, so that no matching need occur for those thresholds, since they are already implicitly included in the couplings. The limit of this procedure as $a \rightarrow \infty$ leads to a formally consistent scheme where no matching or artificial decoupling is used, but due to the failure of decoupling, it requires inclusion of contributions from every particle in the (unknown) fundamental theory. This is the exact situation that caused us to introduce an ADS in the first place, since we did not want unknown and arbitrarily massive fields contributing to every physical observable (written in terms of the ADS scheme charge). It is true that such unknown contributions cancel in relations between observables, but the utility of the intermediate ADS scheme is lost since it's coupling is ill-defined ${ }^{13}$. In many calculations in unphysical schemes such as $\overline{M S}$, the "threshold shifting" approach may be used to yield physical predictions which are arbitrarily accurate by choosing a sufficiently

\footnotetext{
${ }^{12}$ Proper analytic threshold dependence may be defined by $\eta_{p}^{R}(Q / m)$ going to a constant for both small and large $Q / m$. Consider the analytic extension of $\overline{M S}$ into the region of mass thresholds, which we call massive $\overline{M S}$, or $M \overline{M S}$ (similar to that in [27]). This is defined by $\eta_{p}^{M \overline{M S}}\left(Q_{0} / m_{p}\right)=0$ so that the full logarithmic-like functions $L_{p}$ are subtracted and trivially the conditions for smooth threshold dependence are satisfied. Nonetheless, $M \overline{M S}$ has matching errors, which result from the failure of Eq.(3.11) and the subsequent need to construct an ADS from $M \overline{M S}$.

${ }^{13}$ We might as well always write observables in terms of other observables; this is precisely the philosophy of effective charge inspired physical renormalization schemes.
} 
large ' $a$ '. However, the usefulness of this procedure depends on the details of the mass spectrum. There is no universal algorithm that applies to any field theory. The complicated nature of such artificial fixes to the decoupling problem are reflections of the unphysical nature of the schemes and couplings. See [28] for another approach to fixing $\overline{M S}$.

Thus, we have shown that the $\overline{M S}$ and $\overline{D R}$ schemes suffer errors unless one is restricted to observables at energies $Q$ which lie far between mass thresholds. In addition, complicated matching conditions must be applied when crossing thresholds to maintain consistency for such desert scenarios. In principle, these schemes are only valid for theories where all particles have zero or infinite mass, or if one knows the full field content of the underlying physical theory. 


\section{Chapter 4}

\section{Methodology and Selected Results of Precision Gauge Unification}

Given the plethora of unified models and theories of particle physics, it is useful to be able to treat each of these on equal footing in regards to the detailed predictions of gauge-coupling unification. Only a small set of formula suffice to fully describe the running gauge couplings of the Standard Model in any theory at two-loops with all thresholds treated rigorously. Since only partial and model specific results have appeared in the literature, it seems useful to collect here some general results. This chapter represents a synthesis of results from chapter 3 and some additional, more general, results that are not specific to the physical effective charge approach considered in that chapter. The results given below have been derived using the canonical literature $[46,47,48,49]$ and from detailed diagrammatic calculations (see Chapter $6)$. 


\subsection{General Results for the Running Couplings}

\subsubsection{The RGE and thresholds}

The $\beta$ function coefficients are defined by the renormalization group equation (RGE) for the $U(1)_{Y} \times S U(2)_{L} \times S U(3)_{c}$ gauge couplings $\alpha_{i}=g_{i}^{2} /(4 \pi)(i=1,2,3)$ :

$$
\frac{\partial \alpha_{i}}{\partial \log Q^{2}}=-\frac{\alpha_{i}^{2}}{4 \pi}\left(\beta_{i}+\frac{1}{4 \pi} \sum_{j=1}^{3} \beta_{i j} \alpha_{j}+\frac{1}{(4 \pi)^{2}} Y_{4}^{(i)}(F)\right) .
$$

The detailed formula for the one-loop $\beta_{i}$ terms, two-loop gauge terms $\left(\beta_{i j}\right)$, and twoloop Yukawa terms $\left(Y_{4}^{(i)}(F)\right)$ will be considered in succession momentarily. First, the solution and threshold effects will be discussed.

In the $\overline{P T}$ scheme used in this chapter the $\beta_{i}$ are analytic functions of the masses,

$$
\beta_{i} \rightarrow \beta_{i}(Q)=\sum_{p} \beta_{i}^{(p)} \frac{\partial L_{p}\left(Q^{2} / m_{p}^{2}\right)}{\partial \log Q^{2}}
$$

where the $L_{p}$ functions are given exactly in Eq.(3.19). The numerical approximation for $L_{p}$ given in Eq.(3.6) is more than adequate for GUT purposes and leads to

$$
L_{p} \approx \log \left(e_{p}^{\eta}+\frac{Q^{2}}{m_{p}^{2}}\right) \quad \frac{\partial L_{p}\left(Q^{2} / m_{p}^{2}\right)}{\partial \log Q^{2}} \approx \frac{1}{1+\frac{m_{p}^{2}}{Q^{2}} e^{\eta_{p}}},
$$

where $\eta_{p}=8 / 3,5 / 3$, and $40 / 21$ for massive spin $0,1 / 2$, and 1 particles, respectively. In this way all one-loop threshold effects (both logarithmic and subleading) are automatically treated correctly and do not need to be included by hand. Two-loop thresholds are implemented through step-functions. As mentioned earlier, the complete two-loop threshold functions are not available, but have been estimated to give a very small correction to the leading (step-function) two-loop thresholds [41]. 
The perturbative solution of the Eq.(4.1) can be written as

$$
\begin{aligned}
\alpha_{i}^{-1}(Q) & =\alpha_{i}^{-1}\left(Q_{0}\right)+\frac{1}{4 \pi} \sum_{p} \beta_{i}^{(p)}\left[L_{p}\left(\frac{Q}{m_{p}}\right)-L_{p}\left(\frac{Q_{0}}{m_{p}}\right)\right]-\theta_{i}\left(Q, Q_{0}\right) \\
& +\frac{1}{(4 \pi)^{3}} Y_{4}^{(i)}(F) \log \frac{Q^{2}}{Q_{0}^{2}}
\end{aligned}
$$

where the two-loop effects are contained in the terms $\theta_{i}\left(Q, Q_{0}\right)$ in Eq. (3.23).

$$
\theta_{i}\left(Q, Q_{0}\right)=\frac{1}{4 \pi} \sum_{j=1}^{3} \frac{\beta_{i j}}{\beta_{j}} \log \left(\frac{\alpha_{j}(Q)}{\alpha_{j}\left(Q_{0}\right)}\right)
$$

and the approximation has been made that the Yukawa couplings do not run. For the purposes of gauge-coupling unification, this is a valid approximation for the SM and MSSM Yukawa couplings, and can be improved by evaluating $Y_{4}^{(i)}(F)$ at the geometric mean between $Q$ and $Q_{0}$. Numerical techniques are required for an exact solution. In the massless limit Eq.(4.4) becomes

$$
\alpha_{i}^{-1}(Q)=\alpha_{i}^{-1}\left(Q_{0}\right)+\frac{1}{4 \pi}\left(\beta_{i}+\frac{1}{(4 \pi)^{2}} Y_{4}^{(i)}(F)\right) \log \left(\frac{Q^{2}}{Q_{0}^{2}}\right)-\theta_{i}\left(Q, Q_{0}\right)
$$

One can translate to the $\overline{D R}$ scheme coupling $(\bar{\alpha})$ at the one-loop level through the relations (see sections 3.2 .2 and 6.3 .1 for details)

$$
\begin{aligned}
\frac{1}{\alpha_{i}(Q)} & =\frac{1}{\overline{\alpha_{i}}(Q)}+\frac{1}{4 \pi} \sum_{p} \beta_{i}^{(p)}\left(L_{p}\left(\frac{Q^{2}}{m_{p}^{2}}\right)-\log \left(\frac{Q^{2}}{m_{p}^{2}}\right)-\eta_{p}\right) \\
& \approx \frac{1}{\overline{\alpha_{i}}(Q)}-\frac{1}{4 \pi} \sum_{p} \beta_{i}^{(p)} \eta_{p} \\
& \approx \frac{1}{\overline{\alpha_{i}}(Q)}+\frac{1}{4 \pi}\left(-\frac{64}{9} C_{A}+\frac{20}{9} T_{F}+\frac{8}{9} T_{S}\right)
\end{aligned}
$$

where the latter two equations hold in the massless limit and we used the result $\eta_{1}=64 / 33$ for massless vector bosons. From the above results for the one can obtain all of the one-loop threshold corrections in the $\overline{D R}$ scheme. The two-loop relation between the pinch-technique and $\overline{D R}(\overline{M S})$ couplings is derived in Chapter 6 . 


\subsubsection{Group theory basics}

First, we should establish some efficient conventions for the group theory. Every multiplet can be decomposed as a sum of Standard Model multiplets using so-called branching rules. The multiplets given in Eq.(4.9) below are the most commonly occurring, although sometimes others such as $(-2 / 3,1,6)$ must be used. In any case, here we consider the most general case

$$
R=\left(y, R_{2}, R_{3}\right)
$$

where $R_{2}$ and $R_{3}$ are the representations under $S U(2)_{L}$ and $S U(3)_{c}$, respectively. The hypercharge is normalized so that the Standard Model multiplets are

$$
\begin{array}{ccc}
Q=(1 / 6, \mathbf{2}, \mathbf{3}) & u=(2 / 3, \mathbf{1}, \mathbf{3}) & d=(-1 / 3, \mathbf{1}, \mathbf{3}) \\
L=(-1 / 2, \mathbf{2}, \mathbf{1}) & e=(1, \mathbf{1}, \mathbf{1}) & \\
b=(0, \mathbf{1}, \mathbf{1}) & w=(0, \mathbf{3}, \mathbf{1}) & g=(0, \mathbf{1}, \mathbf{8})
\end{array}
$$

The dimension of $R, d(R)=d_{2} d_{3}$, is the product of the dimensions of $R_{2}$ and $R_{3}$. The group invariants defined by

$$
\delta^{a b} T(R)=\operatorname{Tr}\left[T_{R}^{a} T_{R}^{b}\right] \quad C(R)=T_{R}^{a} T_{R}^{a}
$$

are written as

$$
\begin{array}{ll}
T_{3} \equiv T\left(R_{3}\right) & T_{2} \equiv T\left(R_{2}\right) \\
C_{3} \equiv C\left(R_{3}\right) & C_{2} \equiv C\left(R_{2}\right) .
\end{array}
$$

Using the relations

$$
T_{3}=\frac{C_{3} d_{3}}{8} \quad \text { and } \quad T_{2}=\frac{C_{2} d_{2}}{3}
$$

we will write all of our results in terms of the quadratic Casimir invariants $C_{2}, C_{3}$, the hypercharge $y$, and the dimension of the representation $d(R)$. For the most commonly occurring representations, such as the $\mathbf{5}, \mathbf{1 0}$, and $\mathbf{2 4}$ of $S U(5)$ and the 10, 16, and 45 
of $S O(10)$, only the elementary values for fundamental and adjoint reps are needed:

$$
\begin{array}{lll}
C_{2}(1)=0 & C_{2}(\mathbf{2})=3 / 4 & C_{2}(3)=2 \\
C_{3}(1)=0 & C_{3}(3)=4 / 3 & C_{3}(8)=3
\end{array}
$$

For higher dimensional representations, the tables of [49] are very helpful.

\subsubsection{One-Loop $\beta_{i}$ coefficients}

The contribution of a chiral superplet (CS) to the one-loop $\beta$ coefficients is

$$
\beta_{i}^{(C S)}(R)=-d(R)\left(\begin{array}{c}
\frac{3}{5} y^{2} \\
\frac{1}{3} C_{2} \\
\frac{1}{8} C_{3}
\end{array}\right)
$$

from which the results for vector superplets (VS), regular vectors (V), Weyl fermions (F), or complex scalars (S) can be obtained by multiplying by $-3,-11 / 3,2 / 3$, and $1 / 3$, respectively. For the SM we have

$$
\begin{aligned}
& \beta_{i}=-\frac{11}{3} \beta_{i}^{(C S)}(w+g)+\frac{2}{3} N_{g} \beta_{i}^{(C S)}(Q+u+d+L+e)+\frac{1}{3} N_{h} \beta_{i}^{(C S)}(L) \\
&\left(\begin{array}{c}
\beta_{1} \\
\beta_{2} \\
\beta_{3}
\end{array}\right)=\left(\begin{array}{c}
0 \\
\frac{22}{3} \\
11
\end{array}\right)-\frac{4}{3} N_{g}\left(\begin{array}{c}
1 \\
1 \\
1
\end{array}\right)-N_{H}\left(\begin{array}{c}
\frac{1}{10} \\
\frac{1}{6} \\
0
\end{array}\right)=\left(\begin{array}{c}
-\frac{41}{10} \\
\frac{19}{6} \\
7
\end{array}\right)
\end{aligned}
$$

For the MSSM we have

$$
\begin{aligned}
\beta_{i} & =-3 \beta_{i}^{(C S)}(w+g)+N_{g} \beta_{i}^{(C S)}(Q+u+d+L+e)+N_{h} \beta_{i}^{(C S)}(L) \\
\left(\begin{array}{c}
\beta_{1} \\
\beta_{2} \\
\beta_{3}
\end{array}\right) & =\left(\begin{array}{l}
0 \\
6 \\
9
\end{array}\right)-2 N_{g}\left(\begin{array}{l}
1 \\
1 \\
1
\end{array}\right)-N_{H}\left(\begin{array}{c}
\frac{3}{10} \\
\frac{1}{2} \\
0
\end{array}\right)=\left(\begin{array}{c}
-\frac{33}{5} \\
-1 \\
3
\end{array}\right)
\end{aligned}
$$




\subsubsection{Two-Loop $\beta_{i j}$ coefficients}

The two-loop coefficients for various multiplets are given below.

Chiral superplets (CS):

$$
\beta_{i j}^{(C S)}(R)=-d(R)\left(\begin{array}{ccc}
\frac{36}{25} y^{4} & \frac{12}{5} y^{2} C_{2} & \frac{12}{5} y^{2} C_{3} \\
\frac{4}{5} y^{2} C_{2} & \frac{4}{3} C_{2}\left(1+C_{2}\right) & \frac{4}{3} C_{2} C_{3} \\
\frac{3}{10} y^{2} C_{3} & \frac{1}{2} C_{2} C_{3} & \frac{1}{4} C_{3}\left(3+2 C_{3}\right)
\end{array}\right),
$$

Chiral fermions $(\mathrm{F})$ :

$$
\beta_{i j}^{(F)}(R)=-d(R)\left(\begin{array}{ccc}
\frac{18}{25} y^{4} & \frac{6}{5} y^{2} C_{2} & \frac{6}{5} y^{2} C_{3} \\
\frac{2}{5} y^{2} C_{2} & \frac{2}{9} C_{2}\left(10+3 C_{2}\right) & \frac{2}{3} C_{2} C_{3} \\
\frac{3}{20} y^{2} C_{3} & \frac{1}{4} C_{2} C_{3} & \frac{1}{4} C_{3}\left(5+C_{3}\right)
\end{array}\right)
$$

Complex Scalars (S):

$$
\beta_{i j}^{(S)}(R)=-d(R)\left(\begin{array}{ccc}
\frac{36}{25} y^{4} & \frac{12}{5} y^{2} C_{2} & \frac{12}{5} y^{2} C_{3} \\
\frac{4}{5} y^{2} C_{2} & \frac{4}{9} C_{2}\left(1+3 C_{2}\right) & \frac{4}{3} C_{2} C_{3} \\
\frac{3}{10} y^{2} C_{3} & \frac{1}{2} C_{2} C_{3} & \frac{1}{4} C_{3}\left(1+2 C_{3}\right)
\end{array}\right)
$$

SUSY gaugino-fermion-scalar (GFS) Yukawa vertex:

$$
\beta_{i j}^{(G F S)}(R)=d(R)\left(\begin{array}{ccc}
\frac{18}{25} y^{4} & \frac{6}{5} y^{2} C_{2} & \frac{6}{5} y^{2} C_{3} \\
\frac{2}{5} y^{2} C_{2} & \frac{2}{3} C_{2}\left(2+C_{2}\right) & \frac{2}{3} C_{2} C_{3} \\
\frac{3}{20} y^{2} C_{3} & \frac{1}{4} C_{2} C_{3} & \frac{1}{4} C_{3}\left(3+C_{3}\right)
\end{array}\right)
$$

Notice that $-\beta_{i j}^{(C S)}(R)+\beta_{i j}^{(F)}(R)+\beta_{i j}^{(S)}(R)+\beta_{i j}^{(G F S)}(R)=0$, since the contribution of a chiral superplet is just the same as $(F)+(S)+(G F S)$. The vector superplets (VS) for representations $w=(0,3,1)$ and $g=(0, \mathbf{1}, \mathbf{8})$ have

$$
\beta_{i j}^{(V S)}(g)=-\beta_{i j}^{(C S)}(g)=\left(\begin{array}{ccc}
0 & 0 & 0 \\
0 & 0 & 0 \\
0 & 0 & 54
\end{array}\right)
$$


and

$$
\beta_{i j}^{(V S)}(w)=-\beta_{i j}^{(C S)}(w)=\left(\begin{array}{ccc}
0 & 0 & 0 \\
0 & 24 & 0 \\
0 & 0 & 0
\end{array}\right)
$$

while the non-supersymmetric vector multiplets have

$$
\beta_{i j}^{(V)}(g)=\beta_{i j}^{(V S)}(g)-\beta_{i j}^{(F)}(g)=\left(\begin{array}{ccc}
0 & 0 & 0 \\
0 & 0 & 0 \\
0 & 0 & 102
\end{array}\right)
$$

and

$$
\beta_{i j}^{(V)}(w)=\beta_{i j}^{(V S)}(w)-\beta_{i j}^{(F)}(w)=\left(\begin{array}{ccc}
0 & 0 & 0 \\
0 & \frac{136}{3} & 0 \\
0 & 0 & 0
\end{array}\right) .
$$

Let us consider some examples.

In $S U(5)$ and $S O(10)$, the most commonly occurring representations have the following branching rules (where $X=(-5 / 6,2,3)$ ):

$$
\begin{aligned}
& S U(5) \rightarrow S M \quad S O(10) \rightarrow S U(5) \\
& \mathbf{5} \rightarrow d+\bar{L} \quad 10_{v} \rightarrow \mathbf{5}+\overline{\mathbf{5}} \\
& 10 \rightarrow Q+\bar{u}+\bar{e} \quad 16 \rightarrow 10_{v}+\overline{5}+1 \\
& 24 \rightarrow g+w+b+X+\bar{X} \quad 45 \rightarrow 24+10+\overline{10}+1,
\end{aligned}
$$


These lead to the following results for each chiral superplet.

$$
\begin{aligned}
& S U(5) \quad S O(10) \\
& \beta_{i j}^{(C S)}(\mathbf{5})=-\left(\begin{array}{ccc}
\frac{7}{30} & \frac{9}{10} & \frac{16}{15} \\
\frac{3}{10} & \frac{7}{2} & 0 \\
\frac{2}{5} & 0 & \frac{17}{3}
\end{array}\right) \quad \beta_{i j}^{(C S)}\left(\mathbf{1 0}_{v}\right)=-\left(\begin{array}{ccc}
\frac{7}{15} & \frac{9}{5} & \frac{32}{15} \\
\frac{3}{5} & 7 & 0 \\
\frac{4}{5} & 0 & \frac{34}{3}
\end{array}\right) \\
& \beta_{i j}^{(C S)}(\mathbf{1 0})=-\left(\begin{array}{ccc}
\frac{23}{10} & \frac{3}{10} & \frac{24}{5} \\
\frac{1}{10} & \frac{21}{2} & 8 \\
\frac{3}{5} & 3 & 17
\end{array}\right) \quad \beta_{i j}^{(C S)}(\mathbf{1 6})=-\left(\begin{array}{ccc}
\frac{38}{15} & \frac{6}{5} & \frac{88}{15} \\
\frac{2}{5} & 14 & 8 \\
\frac{11}{15} & 3 & \frac{68}{3}
\end{array}\right) \\
& \beta_{i j}^{(C S)}(\mathbf{2 4})=-\left(\begin{array}{ccc}
\frac{25}{3} & 15 & \frac{80}{3} \\
5 & 45 & 16 \\
\frac{10}{3} & 6 & \frac{230}{3}
\end{array}\right) \quad \beta_{i j}^{(C S)}(\mathbf{4 5})=-\left(\begin{array}{ccc}
\frac{194}{15} & \frac{78}{5} & \frac{544}{15} \\
\frac{26}{5} & 66 & 32 \\
\frac{68}{15} & 12 & \frac{332}{3}
\end{array}\right)
\end{aligned}
$$

The results for the $S U(5) 5$ and 10 agree with [50]. However, for the 24 we disagree in the 2,2 and 3,3 entries; the values $\beta_{22}(\mathbf{2 4})=-33$ and $\beta_{33}(\mathbf{2 4})=-68 / 3$ were found in [50]. From the general results of [48] (see also Eq.(6.8)), we have for any chiral superplet the 3,3 term $\beta_{33}=-2 T_{3}\left(3+2 C_{3}\right) d_{2}=-\frac{1}{4} d(R) C_{3}\left(3+2 C_{3}\right)$, from which it follows that $\beta_{33}(g)=-\frac{1}{4}(8)(3)(3+2(3))=-54$ and $\beta_{33}(X)=-\frac{1}{4}(6)(4 / 3)(3+$ $2(4 / 3))=-34 / 3$, which leads to $\beta_{33}(\mathbf{2 4})=\beta_{33}(g)+2 \beta_{33}(X)=-230 / 3$, confirming our result and demonstrating that the incorrect result of [50] for the 3,3 entry is due to neglecting the $g$ component of the $\mathbf{2 4}$. A similar exercise for the 2,2 entry will convince the reader that the value reported here is correct.

The two-loop contributions of any other multiplets can easily be obtained from the general results given above in concert with the branching rules given in Ref.[49].

For the SM the two-loop matrix of coefficients is

$$
\begin{aligned}
\beta_{i j}^{S M} & =\beta_{i j}^{(V)}(w+g)+N_{g} \beta_{i j}^{(F)}(Q+u+d+L+e)+N_{h} \beta_{i j}^{(S)}(L) \\
& =-\left(\begin{array}{ccc}
\frac{199}{50} & \frac{27}{10} & \frac{44}{5} \\
\frac{9}{10} & \frac{35}{6} & 12 \\
\frac{11}{10} & \frac{9}{2} & -26
\end{array}\right)
\end{aligned}
$$


while for the MSSM

$$
\begin{aligned}
\beta_{i j}^{M S S M} & =\beta_{i j}^{(V S)}(w+g)+N_{g} \beta_{i j}^{(C S)}(Q+u+d+L+e)+N_{h} \beta_{i j}^{(C S)}(L) \\
& =-\left(\begin{array}{ccc}
\frac{199}{25} & \frac{27}{5} & \frac{88}{5} \\
\frac{9}{5} & 25 & 24 \\
\frac{11}{5} & 9 & 14
\end{array}\right) .
\end{aligned}
$$

\subsubsection{Two-Loop Yukawa $Y_{4}^{(i)}(F)$ coefficients}

As seen in Eq.(4.1), the Yukawa couplings contribute to the two-loop running of the gauge couplings through the invariant

$$
Y_{4}^{(i)}(F)=\frac{1}{d\left(G_{i}\right)} \operatorname{Tr}\left[C_{i}(F) Y^{\alpha} Y^{\alpha \dagger}\right]
$$

where notation similar to Machacek and Vaughn [46] is used. The Yukawa interactions are of the form $\mathcal{L}_{i n t}=-Y_{I J}^{\alpha} \phi_{\alpha} \psi_{I} \psi_{J}+$ h.c. with real scalars and Weyl fermions. Since it is often tedious to evaluate this invariant for particular theories, an example may be useful. The gaugino-fermion-scalar Yukawa couplings of supersymmetric theories can be written as

$$
\mathcal{L}_{\text {int }}=-\sqrt{2} g T_{\alpha \beta}^{A} \phi_{\alpha} \widetilde{G}_{A} \psi_{\beta}+\text { h.c. },
$$

where the Greek indices $\alpha, \beta, \gamma$ run over the representation $(R)$ of the chiral superplet containing $\phi$ and $\psi(1, \ldots, d(R))$, while $A, B, C$ are the adjoint labels and run from $1, \ldots, d(G)$. Thus the Yukawa matrix is

$$
Y_{I J}^{\alpha}=\sqrt{2} g\left(\begin{array}{cc}
0 & T_{\alpha \beta}^{C} \\
T_{\alpha \gamma}^{B} & 0
\end{array}\right)
$$

with $I$ and $J$ are summed over $\beta, B$ and $\gamma, C$, respectively. This leads to

$$
\left(Y^{\alpha} Y^{\alpha \dagger}\right)_{I J}=2 g^{2}\left(\begin{array}{cc}
C(R) \delta_{\beta \gamma} & 0 \\
0 & T(R) \delta_{B C}
\end{array}\right)
$$


Similarly, the generators

$$
T_{I J}^{A}=\left(\begin{array}{cc}
T_{\beta \gamma}^{A}(R) & 0 \\
0 & T_{B C}^{A}(G)
\end{array}\right)
$$

lead to the Casimir operator

$$
C(F)_{I J}=\left(T^{A} T^{A}\right)_{I J}=\left(\begin{array}{cc}
C(R) \delta_{\beta \gamma} & 0 \\
0 & C(G) \delta_{B C}
\end{array}\right)
$$

Assembling these results we find $Y_{4}(F)=2 g^{2} \frac{1}{d(G)}\left[(C(R))^{2} d(R)+C(G) T(R) d(G)\right]$, which upon using $C(R) d(R)=T(R) d(G)$ leads to the final result

$$
Y_{4}^{(i)}(F)=2 g_{i}^{2} T_{i}(R)\left[C_{i}(R)+C_{i}(G)\right]
$$

From this expression it is relatively straightforward to derive the matrix in Eq.(4.20).

For the top, bottom, and $\tau$ Yukawa couplings of the SM and MSSM the result is

$$
\begin{aligned}
& Y_{4}^{(i)}(F)=y_{t}^{2}\left(\begin{array}{c}
\frac{17}{10} \\
\frac{3}{2} \\
2
\end{array}\right)+y_{b}^{2}\left(\begin{array}{c}
\frac{1}{2} \\
\frac{3}{2} \\
2
\end{array}\right)+y_{\tau}^{2}\left(\begin{array}{c}
\frac{3}{2} \\
\frac{1}{2} \\
0
\end{array}\right) \quad \text { SM } \\
& Y_{4}^{(i)}(F)=y_{t}^{2}\left(\begin{array}{c}
\frac{26}{5} \\
6 \\
4
\end{array}\right)+y_{b}^{2}\left(\begin{array}{c}
\frac{14}{5} \\
6 \\
4
\end{array}\right)+y_{\tau}^{2}\left(\begin{array}{c}
\frac{18}{5} \\
2 \\
0
\end{array}\right) \quad \text { MSSM }
\end{aligned}
$$

\subsection{Additional Corrections}

\subsubsection{Initial value re-calibration of the QED coupling}

As mentioned below Eq.(3.22), the QED coupling $\alpha\left(M_{Z}\right)$ is extracted from the zero-momentum limit $\alpha^{-1}(0) \approx 137.036$ limit by running with only the SM particles included. When there are light charged ( $\mathrm{s}$ )particles not much heavier than $M_{Z}$, these (s)particles induce additional corrections to the initial value $\alpha\left(M_{Z}\right)$ used 
for GUT purposes. Since it would be unpleasant to quote different $\alpha\left(M_{Z}\right)$ for each model, we instead consider $\alpha\left(M_{Z}\right)$ to be the model-independent value obtained by neglecting all non-SM particles, and include the corrections explicitly through $\alpha^{-1}\left(M_{Z}\right) \rightarrow \alpha^{-1}\left(M_{Z}\right)+\delta_{\alpha}$ where

$$
\dot{\delta_{\alpha}}=\frac{1}{4 \pi} \sum_{p \notin S M} \beta_{\gamma \gamma}^{(p)}\left[L_{p}\left(\frac{M_{Z}}{m_{p}}\right)-\eta_{p}\right] .
$$

This leads to the corrections

$$
\begin{aligned}
& \delta\left(\alpha_{1}^{-1}\left(M_{Z}\right)\right)=\frac{3}{5} c_{w}^{2}\left(M_{Z}\right) \delta_{\alpha} \\
& \delta\left(\alpha_{2}^{-1}\left(M_{Z}\right)\right)=s_{w}^{2}\left(M_{Z}\right) \delta_{\alpha} .
\end{aligned}
$$

\subsubsection{Gaugino-Higgsino Mixing}

Generally the mass eigenstates do not correspond to the $S U(2)_{L} \times U(1)_{Y}$ eigenstates, which can lead to problems in properly treating thresholds. In the approach used in this thesis, these effects are naturally accounted for since the $\alpha_{1}$ and $\alpha_{2}$ running couplings are defined in terms of the $\gamma \gamma$ and $\gamma Z$ self-energies, as seen in Eq.(3.18). To illustrate these corrections, consider the chargino mass matrix

$$
\begin{array}{cc}
X=\left(\begin{array}{cc}
M_{2} & \sqrt{2} s_{\beta} M_{w} \\
\sqrt{2} c_{\beta} M_{w} & \mu
\end{array}\right) \quad U^{*} X V^{-1}=\left(\begin{array}{cc}
M\left(\chi_{1}^{ \pm}\right) & 0 \\
0 & M\left(\chi_{2}^{ \pm}\right)
\end{array}\right) \\
V=\left(\begin{array}{cc}
\cos \phi_{+} & \sin \phi_{+} \\
-\sin \phi_{+} & \cos \phi_{+}
\end{array}\right) & U=\left(\begin{array}{cc}
\cos \phi_{-} & \sin \phi_{-} \\
-\sin \phi_{-} & \cos \phi_{-}
\end{array}\right)
\end{array}
$$

Calculating the chargino contribution to the running couplings in the mass eigenstate basis $\left(\gamma \gamma\right.$ and $\gamma Z$ ) leads to (defining $s_{ \pm}=\sin \phi_{ \pm}$and $c_{ \pm}=\cos \phi_{ \pm}$)

$$
\begin{array}{ll}
\beta_{1}\left(\chi_{1}^{ \pm}\right)=-\frac{1}{5}\left(s_{+}^{2}+s_{-}^{2}\right) & \beta_{2}\left(\chi_{1}^{ \pm}\right)=-\frac{4}{3}+\frac{1}{3}\left(s_{+}^{2}+s_{-}^{2}\right) \\
\beta_{1}\left(\chi_{2}^{ \pm}\right)=-\frac{1}{5}\left(c_{+}^{2}+c_{-}^{2}\right) & \beta_{2}\left(\chi_{2}^{ \pm}\right)=-\frac{4}{3}+\frac{1}{3}\left(c_{+}^{2}+c_{-}^{2}\right) .
\end{array}
$$


Added together we have the expected results $\beta_{1}\left(\chi_{1}^{ \pm}+\chi_{2}^{ \pm}\right)=-\frac{2}{5}$ and $\beta_{2}\left(\chi_{1}^{ \pm}+\chi_{2}^{ \pm}\right)=-2$. However, the splitting in the masses of the charginos induces a decoupling correction between the two chargino masses which involves the above mixing angles $\phi_{ \pm}$. Similar corrections arise for squarks and sleptons when the $L$ and $R$ components have a significant mass splitting. All of these mass-mixing corrections are usually numerically small, but possibly relevant in the future. Similar finite non-logarithmic threshold corrections have been considered in the conventional $\overline{D R}$ framework in Refs. [23, 51].

\subsubsection{Non-renormalizable operators (NROs) from Gravity}

Here we will only discuss the leading dimension five operator of $S U(5)$ first introduced in Refs. $[52,53]$ and later considered in Refs. [54, 55] :

$$
\delta \mathcal{L}=-\frac{1}{2} \frac{\eta}{M_{P l}} \operatorname{Tr}\left[F_{\mu \nu} \Sigma_{24} F^{\mu \nu}\right]
$$

where $F_{\mu \nu}$ is the $S U(5)$ field strength tensor, the field $\Sigma_{24}$ is responsible for breaking $S U(5)$, and $\eta$ is the unknown dimensionless coefficient. Expanding in terms of SM gauge fields leads to

$$
\begin{aligned}
& \mathcal{L}_{\text {kin }}=-\frac{1}{4} G_{\mu \nu}^{a} G_{a}^{\mu \nu}\left(1+2 \delta_{N R O}\right)-\frac{1}{4} W_{\mu \nu}^{i} W_{i}^{\mu \nu}\left(1-3 \delta_{N R O}\right)-\frac{1}{4} B_{\mu \nu} B^{\mu \nu}\left(1-\delta_{N R O}\right) \\
& \text { where } \quad \delta_{N R O}=\frac{\sqrt{2}}{5} \frac{\eta M_{X}}{g_{U} M_{P l}},
\end{aligned}
$$

where $M_{P l} \approx 2.4 \times 10^{18} \mathrm{GeV}$ is the reduced Planck mass, $M_{X} \sim 2 \times 10^{16} \mathrm{GeV}$ is the mass of the heavy vector bosons of $S U(5)$, and $g_{U}$ is the unification scale coupling. This modifies the unification condition to

$$
\left(1+2 \delta_{N R O}\right) \alpha_{3}\left(M_{U}\right)=\left(1-3 \delta_{N R O}\right) \alpha_{2}\left(M_{U}\right)=\left(1-\delta_{N R O}\right) \alpha_{1}\left(M_{U}\right)
$$

and leads to corrections given below. The supersymmetric version of the above operator was analyzed in [55] and shown to give corrections to the weak scale gaugino mass relations. 


\subsection{Methodology and Summary of Corrections}

The formula given in the above sections suffice to numerically evaluate the running couplings for most any four-dimensional model. However, in order to better understand the nature of the various corrections it is useful to solve the GUT RGE equations in Eq.(4.1) analytically. The results here hold for the pinch-technique effective charges, which have qualitatively different unification behavior compared with the conventional $\overline{D R}(\overline{M S})$ couplings, as discussed in section 3.4 and shown in Fig.(3.6).

We will impose the following universal (asymptotic) boundary condition at a scale $M_{U}$ much greater than both the light (L) MSSM spectrum and the heavy $(\mathrm{H})$ particles at the GUT scale :

$$
\alpha_{1}\left(M_{U}\right)=\alpha_{2}\left(M_{U}\right)=\alpha_{3}\left(M_{U}\right) \equiv \alpha_{G}\left(M_{U}\right)
$$

leads to

$$
\vec{z}=\vec{u}-\vec{\beta}_{G} l_{U}+\vec{\beta}_{H} l_{X}+\vec{\Delta}
$$

where we have adopted the efficient three-vector notation :

$$
\begin{aligned}
\vec{z} & =\left(\alpha_{1}^{-1}\left(M_{z}\right), \alpha_{2}^{-1}\left(M_{z}\right), \alpha_{3}^{-1}\left(M_{z}\right)\right) \\
\vec{u} & =\alpha_{G}^{-1}\left(M_{U}\right)(1,1,1) \\
\vec{\beta}_{G} & =\vec{\beta}_{L}+\vec{\beta}_{H}=\beta_{G}(1,1,1) \\
l_{U} & =\frac{1}{2 \pi} \log \left(\frac{M_{U}}{M_{z}}\right) \\
l_{X} & =\frac{1}{2 \pi}\left(\log \left(\frac{M_{X}}{M_{z}}\right)+1\right)
\end{aligned}
$$

and

$$
\vec{\Delta}=\vec{\Delta}_{L}+\vec{\Delta}_{\alpha}+\vec{\delta}_{H}+\vec{\Delta}_{H}+\vec{\theta}_{2 \text { loop }}+\vec{\Delta}_{Y u k}+\vec{\Delta}_{N R O}
$$

Here $\overrightarrow{\beta_{L}}$ is the vector of one-loop $\beta$ coefficients for the light $(L)$ particles, typically those of the MSSM. Analogously, $\overrightarrow{\beta_{H}}$ is the vector of one-loop $\beta$ coefficients for the heavy $(\mathrm{H})$ particles at the GUT scale. Intermediate scale particles may be included in either $\mathrm{L}$ or $\mathrm{H}$. By the unification assumption the sum of these must be universal 
$\vec{\beta}_{G}=\vec{\beta}_{L}+\vec{\beta}_{H}=\beta_{G}(1,1,1)$. The scale $M_{X}$ is some conveniently chosen scale typical of the heavy spectrum, usually the mass of the heavy gauge bosons. All predictions are independent of this choice.

The correction terms are

- Light thresholds (leading-log and finite analytic)

$$
\vec{\Delta}_{L}=\sum_{l \in L} \frac{1}{4 \pi} \vec{\beta}_{l}\left(L_{l}\left(\frac{M_{Z}}{m_{l}}\right)-\log \frac{M_{Z}^{2}}{m_{l}^{2}}\right)
$$

- Initial value re-calibration for QED coupling (Eq.(4.37))

$$
\vec{\Delta}_{\alpha}=-\sum_{p \notin S M} \frac{1}{4 \pi}\left(\frac{3}{5} c_{w}^{2}\left(M_{z}\right), s_{w}^{2}\left(M_{z}\right), 0\right) \delta_{\alpha}
$$

- Leading-log heavy thresholds

$$
\vec{\delta}_{H}=\sum_{h \in H} \frac{1}{4 \pi} \vec{\beta}_{h} \log \frac{m_{h}^{2}}{M_{X}^{2}}
$$

- Subleading (analytic) heavy thresholds

$$
\vec{\Delta}_{H}=\sum_{h \in H} \frac{1}{4 \pi} \vec{\beta}_{h}\left(L_{h}\left(\frac{M_{U}}{m_{h}}\right)-\log \frac{M_{U}^{2}}{m_{h}^{2}}\right)
$$

- Two-loop corrections with thresholds at intermediate scales $\mu_{1}<\ldots<\mu_{N}$ included

$$
\vec{\theta}_{2 \text { loop }}=\vec{\theta}\left(M_{X}, \mu_{N}\right)+\sum_{n=1}^{N-1} \vec{\theta}\left(\mu_{n+1}, \mu_{n}\right)+\vec{\theta}\left(\mu_{1}, M_{z}\right)
$$

where $\theta_{i}\left(Q, Q_{0}\right)$ given in Eq.(4.5) uses the $\beta_{i}$ and $\beta_{i j}$ appropriate between each threshold. 
- Yukawa coupling two-loop corrections

$$
\vec{\Delta}_{Y u k}=-\frac{1}{(4 \pi)^{3}} \vec{Y}_{4}(F) \log \frac{M_{X}^{2}}{M_{z}^{2}}
$$

- Non-renormalizable operators induced from gravity (see section 4.2.3)

$$
\vec{\Delta}_{N R O}=(-2,3,1) \frac{\delta_{N R O}}{\alpha_{G U T}}
$$

Given the assumption of unification, the two independent equations in Eq.(4.44) yield two predictions among the input parameters. These predictions can most easily be obtained through the use of traceless projection operators [54] $\vec{P}$ on Eq. (4.45). For example, if the light spectrum is the MSSM then $\vec{P}\left(\alpha_{3}\right)=(5 / 7,-12 / 7,1)$ applied to Eq.(4.45) leads to

$$
\frac{1}{\alpha_{3}\left(M_{z}\right)}=z_{3}=-\frac{5}{7} z_{1}+\frac{12}{7} z_{2}-\vec{P}\left(\alpha_{3}\right) \cdot \vec{\Delta}
$$

which expresses the strong coupling prediction in a form that allows one explicitly see the effects of the various correction terms in $\vec{\Delta}$.

\subsection{Some examples}

The following figures have been obtained using the methodology described above using the pinch-technique effective charges. The experimental value is taken to be (see Table 3.2) $\alpha_{3}^{\overline{P T}}+\left(M_{z}\right)=0.140 \pm 0.005$, which corresponds to $\alpha_{3}^{\overline{M S}}\left(M_{z}\right)=0.118 \pm 0.0036$. Results similar to those given below can be obtained in the $\overline{D R}$ approach.

Our starting point is the post-WMAP benchmark scenarios for the supersymmetric parameter space given in Ref.[56], with all high scale threshold effects excluded. As seen in Fig.(4.1), the MSSM does not unify within the experimental tolerances. Nevertheless, a variety of methods are available to salvage gauge-coupling unification.

First, in Fig.(4.2), the two-loop predictions of Split Supersymmetry [57][58] are given. Clearly, values of the scalar masses $M_{S}$ above roughly $10^{8} \mathrm{GeV}$ are preferred. 
This is also the preferred range for solving problems related to flavor violation, $\mathrm{CP}$ violation, and proton decay in the usual low energy supersymmetry framework.

Another possibility is that GUT scale threshold corrections help the couplings unify. As seen in Eqs. $(4.50,3.33)$, this requires a large splitting in the heavy spectrum, or large representations which have large group theory factors. In the minimal supersymmetric $S U(5)$ model with $\mathbf{2 4}_{\mathbf{H}}, \mathbf{5}_{\mathbf{H}}$, and $\overline{\mathbf{5}_{\mathbf{H}}}$, there are no large representations. Therefore, the splitting must be large, and it turns out Higgs triplet must have a mass much less than $10^{17} \mathrm{GeV}$, in violation of proton decay constraints. This is illustrated in Fig.(4.3). In contrast, heavy threshold effects in some $S O(10)$ models tend to work much better [59], with corrections typically of the correct sign and correct order of magnitude.

Another option, which may even salvage the minimal SUSY SU(5) model [55], is that gravitationally induced non-renormalizable operators (NROs) will fix the gaugecoupling unification. This possibility is illustrated in Fig.(4.4), where the effects of the leading dimension five operator discussed in section 4.2 .3 are included. The coefficient $\eta$ cannot be derived without a complete theory of quantum gravity, but is expected to be of order one. As discussed in Ref.[55], the interplay between gauge-coupling unification and weak-scale gaugino masses may provide a testable scenario involving NROs. 


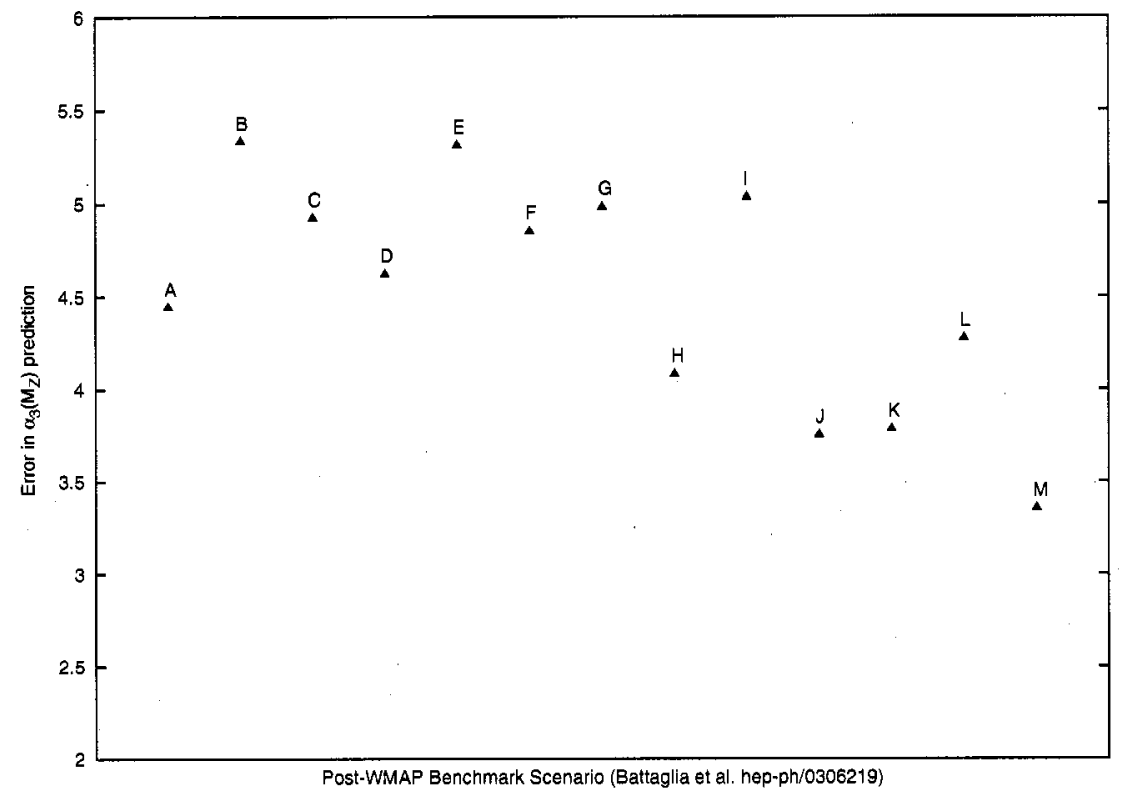

Figure 4.1: MSSM Benchmarks The error in the prediction for the strong coupling, in standard deviations, for each post-WMAP benchmark scenario of Ref.[56].

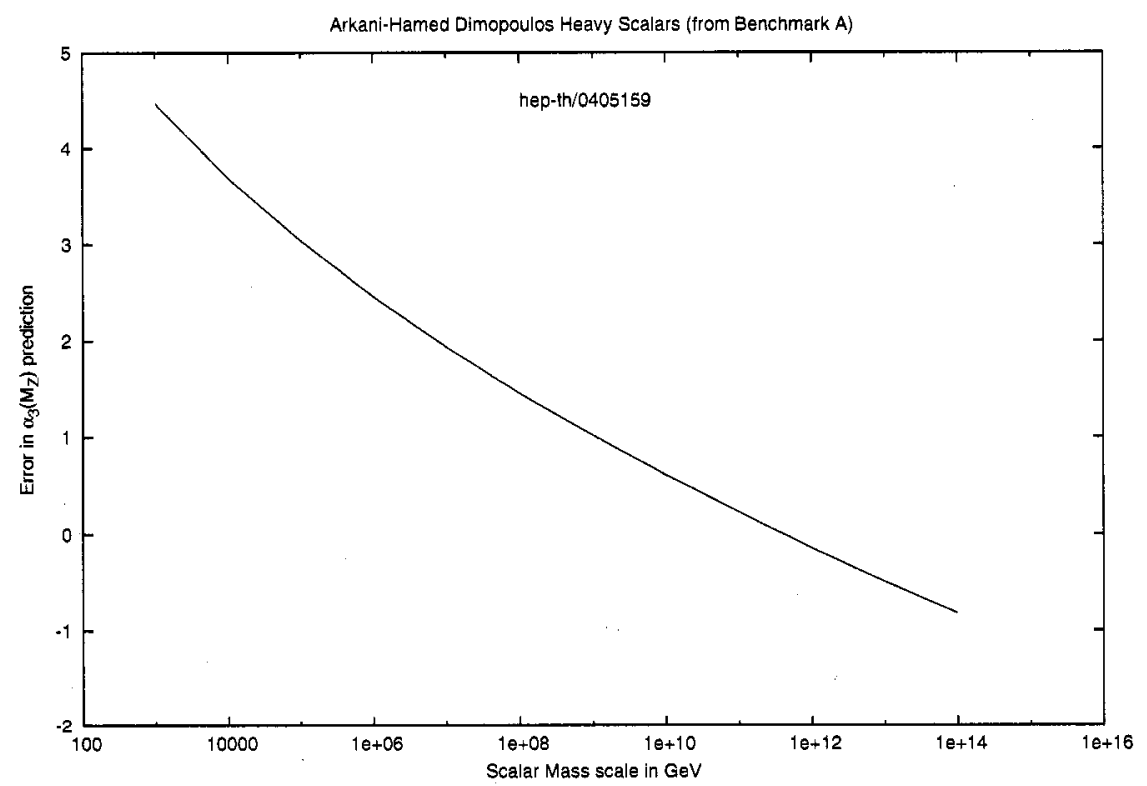

Figure 4.2: Split Supersymmetry For scalar masses $M_{S}>10^{8} \mathrm{GeV}$, the strong coupling predictions are in better agreement with the data compared to the MSSM (Fig.(4.1)). The y-axis is the error in the $\alpha_{s}\left(M_{z}\right)$ prediction, in standard deviations. 


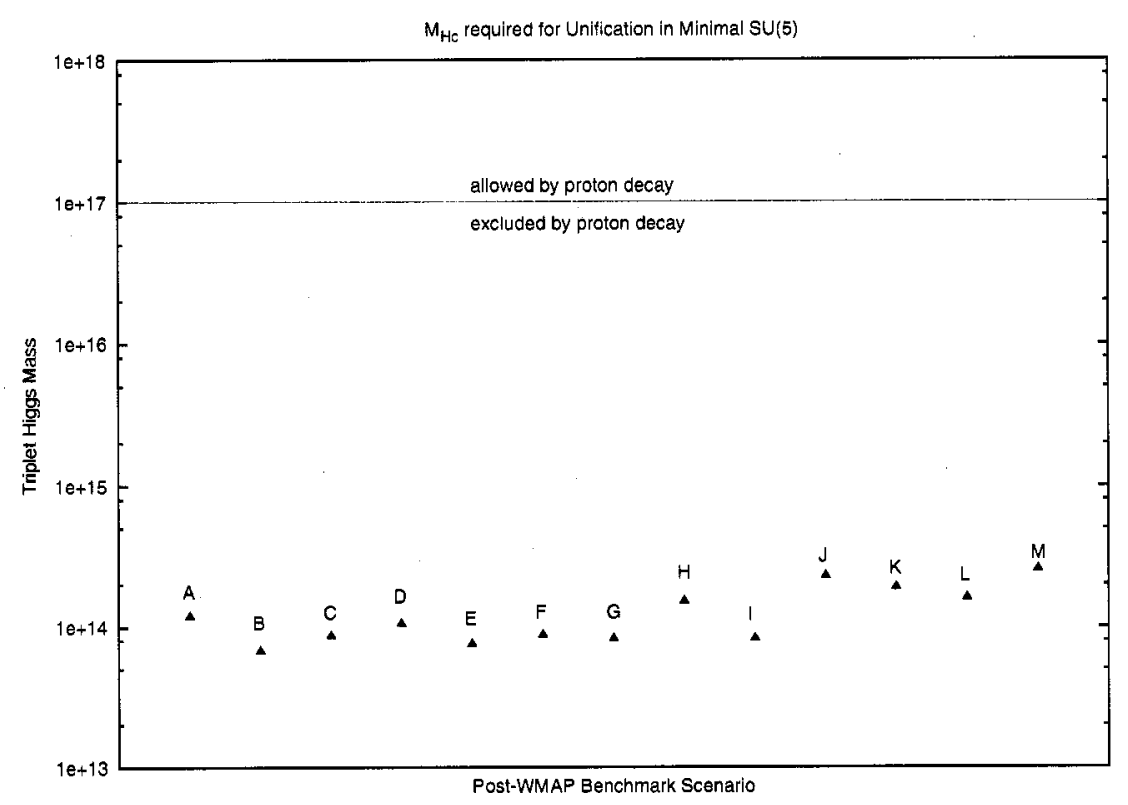

Figure 4.3: Heavy Thresholds The value of the Higgs triplet mass required for consistent gauge coupling unification in the minimal supersymmetric $S U(5)$ model.

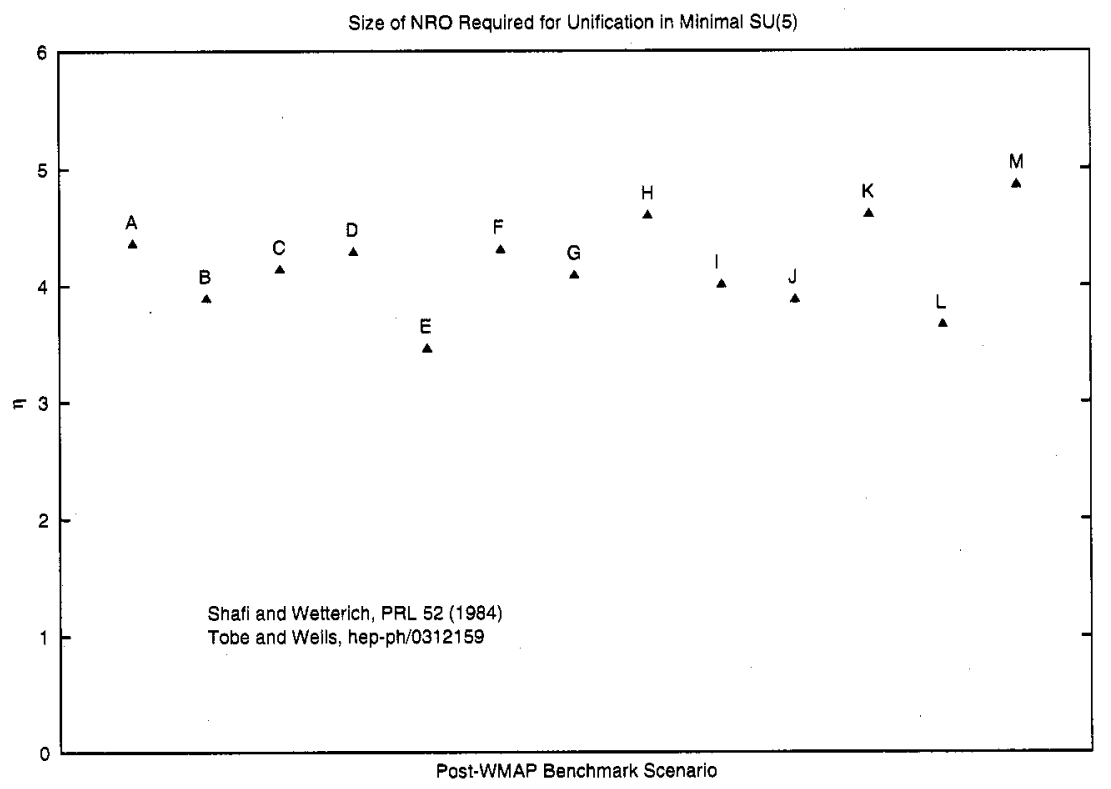

Figure 4.4: Gravitational corrections The size of $\eta$, the dimensionless coefficient of the NRO discussed in section 4.2.3, needed for unification. 


\section{Chapter 5}

\section{The Form Factors of the Gauge Invariant Three Gluon Vertex}

\subsection{Introduction: Gauge-Invariant Green's Func- tions}

The main purpose of this chapter is to analyze the structure of the gauge-invariant three-gluon vertex [60], calculate the fourteen form factors at one loop, and outline some of the phenomenological applications. Before proceeding, it is worthwhile to review the motivation and current status of gauge-invariant Green's functions.

In the conventional formulation of gauge field theories, the manifest gauge-invariance of the original action is lost upon quantization, simply because one has to fix a gauge in order to perform calculations. Generically, Greens's functions are gauge-dependent and thus not physical by themselves. Only the particular combinations of Green's functions which form physical observables must be gauge-invariant. In many theoretical studies, however, one would like to consider individual Green's functions and extract physical meaning from them [30]. For example, studies of the infrared behavior of gauge theory using Dyson-Schwinger equations [61] often rely on gaugedependent truncation schemes which one hopes are not too brutal. The existence of gauge-invariant two-point functions is crucial for defining meaningful resummed 
propagators [34], particularly near threshold, for the construction of effective charges [32], for a postulated dressed-skeleton expansion of QCD [62], and for justifying renormalon analyses [63].

Thus there is strong motivation for gauge-invariant Green's functions with physical content. We will now briefly discuss the relationship between three different approaches to gauge-invariant Green's functions : (1) the Pinch Technique (PT), (2) the Background Field Method (BFM), and (3) the $\star$ effective Lagrangian scheme of Kennedy and Lynn. All three approaches will lead to the same Green's functions.

The pinch technique (PT) was first constructed by Cornwall [30] in order to study gauge-invariant Dyson-Schwinger equations and dynamical gluon mass generation, but the approach is much more generally applicable. In the PT approach, unique gauge-invariant Green's functions are constructed by explicitly rearranging Feynman diagrams using elementary Ward identities (WI) as the guiding principle. Longitudinal momenta from triple-gauge-boson vertices and gauge propagators inside of loops hit other vertices and thus generate inverse propagators (via WI's), which, in turn, cancel (or pinch) some internal propagators. In this way, certain parts of Green's functions are reduced to parts of lower $n$-point functions and should properly be included in the latter.

As an example of the PT, consider the gluon (or massive gauge boson) self-energy. The conventional self-energy is gauge-dependent and physically meaningless by itself. However, when embedded in any physical process, there will be associated parts of vertex and box graphs which undergo the reduction described above and thus have the same tensor and kinematic structure as the gluon propagator. These pinched parts are then added to the conventional gauge-dependent self-energy, yielding a gauge-invariant self-energy and gluon propagator that has the correct asymptotic UV behavior dictated by the renormalization group equation. The resulting two-point function has numerous positive attributes [34][31][32][64][65], including uniqueness, resummability, analyticity, unitarity, and a natural relation to optical theorem, from which it can also be derived [34][33].

Resumming these two-point functions leads to physical effective charges, ála Grunberg [11], which can be extended to the supersymmetric case and leads to an analytic 
improvement of gauge coupling unification with smooth threshold behavior [1].

This method has been applied to a variety of Green's functions [60][66][67] [68][69], with applications to electroweak phenomenology [70][71]. In particular, the gaugeinvariant three-gluon vertex was first constructed in [60] to one-loop order, where the authors showed that the vertex satisfies a relatively simple abelian-like Ward identity. However, the integrals were not evaluated, so that little could be said about the individual form factors except that the UV divergent term in the tree level tensor structure is correct. The main motivation of this chapter is to extend this work by evaluating the integrals for the fourteen form factors, and expressing the results in a convenient tensor basis for phenomenological applications. In doing so, an interesting structure emerges, in which the contributions of gluons $(G)$, quarks $(Q)$, and scalars $(S)$ are intimately related. These relations are closely linked to supersymmetry and conformal symmetry, and in particular the $N=4$ non-renormalization theorems. For all form factors $F$ in dimensions $d$, we find that

$$
F_{G}+4 F_{Q}+(10-d) F_{S}=0
$$

which encodes the vanishing contribution of the $N=4$ supermultiplet in four dimensions. Similar relations have been found in the context of supersymmetric scattering amplitudes [72][73]. In Appendix E, the effects of internal masses are discussed, and the above sum rule becomes modified

$$
F_{M G}+4 F_{M Q}+(9-d) F_{M S}=0
$$

for internal massive gauge bosons (MG), fermions (MQ), and scalar (MS). The external gluons remain massless and unbroken, so the internal gauge bosons might be the heavy $X_{\mu}, Y_{\mu}$ bosons of $S U(5)$, for example. In [74], supersymmetric relations were found for electroweak gauge boson four-point scattering amplitudes.

The PT method has been explicitly extended beyond one-loop [35][36][75], has recently been proven to exist to all orders in perturbation theory [37][38][39][76], and interestingly, each Green's function is equal to the corresponding Green's function of the Background Field Method (BFM) in quantum Feynman gauge $\xi_{Q}=1$, a 
result suggested in $[77][78]$. Heuristically, this is due to the fact that there are no longitudinal (pinching) momenta in the gauge propagator or the elementary vertices in this special gauge.

The Background Field Method (BFM) [79] constructs manifestly gauge invariant Green's functions in the following way. First, the field variable $(A)$ in the path integral is separated into a background $(B)$ and quantum $(Q)$ field, $A=B+Q$. Only the quantum field $Q$ propagates in loops, since it is a variable of functional integration. In contrast, the background field $B$ appears only in external legs. By judiciously choosing the gauge-fixing function, one arrives at an effective action which remains manifestly invariant under background field gauge-transformations $\delta B_{\mu}^{a}=-f^{a b c} \omega^{b} B_{\mu}^{c}+\frac{1}{g} \partial_{\mu} \omega^{a}$. Furthermore, derivatives of the BFM effective action with respect to the background field $B$ yield the same 1PI Green's functions as the conventional effective action with a nonstandard gauge-fixing. Thus, it can be shown [79] that the correct S-matrix is obtained by sewing together trees composed of 1PI Green's functions of $B$ fields. In doing so, one can fix the gauge of $B$, which propagates only at tree level, independently of the gauge fixing of $Q$. For example, convenient non-covariant gauges might be used for the trees while BFM Feynman gauge $\xi_{Q}=1$ (BFMFG) can be used for the loops.

The correspondence between the PT and BFM is not surprising, since the BFM is a formulation of gauge theory where Green's functions of the gauge field are manifestly (background) gauge-invariant. Although this is true for all values of the quantum gauge-fixing parameter $\xi_{Q}$, it is only for the special value $\xi_{Q}=1$ that the BFM Green's functions also have the correct kinematic structure of the irreducible PT. Green's functions. Alternatively, it has been shown [78] that applying the PT algorithm to the BFM for $\xi_{Q} \neq 1$ leads back to the canonical $\left(\xi_{Q}=1\right)$ PT Green's functions.

Finally, in the $\star$ scheme of Kennedy and Lynn [20], a gauge-invariant effective Lagrangian was constructed for electroweak four-fermion processes by explicitly rearranging the one loop corrections. As in the pinch technique, vertex parts must be added to would-be two point functions to yield genuine two-point functions. One particular motivation is that fact that the photon acquires a spurious mass from its mixing with $Z^{0}, \Pi_{\gamma Z}\left(q^{2}=0\right) \neq 0$, unless the correct vertex parts are added. The 
resulting effective charges, $\alpha_{\star}\left(q^{2}\right)$ and $s_{\star}^{2}\left(q^{2}\right)$ are in fact precisely equal to the corresponding pinch-technique effective charges at one loop, including all finite terms and threshold dependence [31].

Thus, all three methods for constructing physical gauge-invariant Green's functions lead to the same results, which in this chapter will be referred to as either PT or PT/BFMFG Green's functions.

The organization of this chapter is as follows. In section 5.2, we will discuss the general structure of the gauge-invariant three-gluon vertex, which is constrained by the Ward identity and Bose symmetry. Two convenient tensor bases and their relation are discussed. In section 5.3, the main results of this chapter are given. First, the nontrivial supersymmetric relations between the gluon, quark, and scalar contributions to each form factor are discussed. The explicit results for the form factors in the case of massless internal particles are given in two different bases, with the full mass effects relegated to Appendix E. In section 5.4, we briefly discuss the phenomenological application to physical scattering prócesses, where we derive an effective coupling for the three-gluon vertex, $\widetilde{\alpha}\left(k_{1}^{2}, k_{2}^{2}, k_{3}^{2}\right)$, and an effective scale, $Q_{e f f}^{2}\left(k_{1}^{2}, k_{2}^{2}, k_{3}^{2}\right)$, both of which depend on three distinct gluon virtualities. In section 5.5, the phenomenological effects of internal masses are discussed. A complicated threshold and pseudo-threshold structure emerges. Furthermore, a three-scale effective number of flavors $N_{F}\left(k_{1}^{2} / M^{2}, k_{2}^{2} / M^{2}, k_{3}^{2} / M^{2}\right)$ is defined. Conclusions are given in section 5.6. In Appendix A, a brief outline of the calculational method is given, and some basic oneand two-point integrals are given. Appendix B is devoted to a thorough discussion of the massive triangle integral, and analytic continuations are given for each kinematic region. Appendix C collects some useful results for special functions. Appendix D explains the corrections to the form factors when a supersymmetric regularization is used. Finally Appendix E gives explicitly the corrections to the form factors arising from internal massive gauge bosons, fermions, and scalars. 


\subsection{General Structure of the Three-Gluon Vertex}

\subsubsection{Symmetries}

One of the most important aspects of the gauge-invariant three-gluon vertex discussed in this chapter is the relatively simple Ward identity it satisfies, which has the same form as the Ward ID satisfied by the tree level vertex. This was proven at one-loop in the original paper by Cornwall and Papavassiliou [60] using the explicit one-loop result, which is the gluon part of Eqs. $(5.17,5.18)$ below. It is straightforward to show that the fermion and scalar parts also satisfy the same Ward identity (just as in QED). Furthermore, the equivalence of the BFMFG and PT to all orders [37] allows one to write the Ward identity satisfied by the three-gluon vertex to all orders as

$p_{3}^{\mu_{3}} \Gamma_{\mu_{1} \mu_{2} \mu_{3}}^{a b c}\left(p_{1}, p_{2}, p_{3}\right)=f^{a d c}\left(t_{\mu_{1} \mu_{2}}\left(p_{2}\right) \delta^{d b}+\Pi_{\mu_{1} \mu_{2}}^{d b}\left(p_{2}\right)\right)-f^{a d c}\left(t_{\mu_{1} \mu_{2}}\left(p_{1}\right) \delta^{d a}+\Pi_{\mu_{1} \mu_{2}}^{d a}\left(p_{1}\right)\right)$,

plus two other equations which are cyclic permutations. The transverse tensor $t_{\mu \nu}(p)=$ $p^{2} g_{\mu \nu}-p_{\mu} p_{\nu}$ comes from the tree level term. Here all momenta are defined to be incoming and all labels are defined in counter-clockwise fashion, as shown in Fig. 5.1. This Ward identity represents a great simplification compared to the usual SlavnovTaylor identities satisfied by the conventional gauge-dependent three-gluon vertex, which involves the gluon propagator, the ghost propagator, and the ghost-ghostgluon vertex function. The self-energy function in the above equation is not the usual gauge-dependent self-energy, but rather the gauge-invariant pinch-technique self-energy, which is the only self-energy discussed in this chapter. An immediate consequence is that the longitudinal $(L)$ part of the vertex, defined as the part which contributes to the above Ward ID, must have only the antisymmetric color factor $f^{a b c}$ so long as gluons conserve the color charge, $\Pi_{\mu \nu}^{a b}(q)=\delta^{a b} \Pi_{\mu \nu}(q)$. As far as we know, the transverse $(T)$ part of the vertex (defined by $p_{3}^{\mu_{3}} \Gamma_{\mu_{1} \mu_{2} \mu_{3}}^{a b c(T)}\left(p_{1}, p_{2}, p_{3}\right)=0$ ) is not required to be proportional to $f^{a b c}$, but may in principle contain $d^{a b c}$ terms. Nevertheless, no such terms appear at one or two loop order, and so in the subsequent 


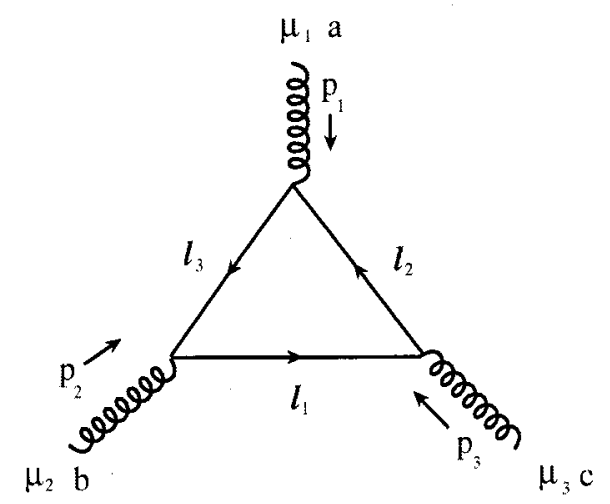

Figure 5.1: The notation and loop momentum routing used throughout this chapter. The internal particle could be a gauge boson, ghost, quark, or scalar.

discussion we take

$$
\Gamma_{\mu_{1} \mu_{2} \mu_{3}}^{a b c}\left(p_{1}, p_{2}, p_{3}\right)=f^{a b c} \Gamma_{\mu_{1} \mu_{2} \mu_{3}}\left(p_{1}, p_{2}, p_{3}\right),
$$

in terms of which the Ward identity becomes

$$
p_{3}^{\mu_{3}} \Gamma_{\mu_{1} \mu_{2} \mu_{3}}\left(p_{1}, p_{2}, p_{3}\right)=t_{\mu_{1} \mu_{2}}\left(p_{2}\right)\left(1+\Pi\left(p_{2}^{2}\right)\right)-t_{\mu_{1} \mu_{2}}\left(p_{1}\right)\left(1+\Pi\left(p_{1}^{2}\right)\right) .
$$

Bose symmetry, the fact that 3 identical particles are entering the vertex, and the properties of $f^{a b c}$ imply definite properties of $\Gamma_{\mu_{1} \mu_{2} \mu_{3}}\left(p_{1}, p_{2}, p_{3}\right)$ under the interchange of labels. In particular, defining the five elements of the permutation group $\mathcal{S}_{3}$ to act by

$$
\begin{array}{cl}
g_{123}=\left(\begin{array}{c}
\left(\mu_{1}, p_{1}\right) \rightarrow\left(\mu_{2}, p_{2}\right) \\
\left(\mu_{2}, p_{2}\right) \rightarrow\left(\mu_{3}, p_{3}\right) \\
\left(\mu_{3}, p_{3}\right) \rightarrow\left(\mu_{1}, p_{1}\right)
\end{array}\right) & g_{12}=\left(\begin{array}{c}
\left(\mu_{1}, p_{1}\right) \rightarrow\left(\mu_{2}, p_{2}\right) \\
\left(\mu_{2}, p_{2}\right) \rightarrow\left(\mu_{1}, p_{1}\right)
\end{array}\right) \\
g_{23}=\left(\begin{array}{c}
\left(\mu_{2}, p_{2}\right) \rightarrow\left(\mu_{3}, p_{3}\right) \\
\left(\mu_{3}, p_{3}\right) \rightarrow\left(\mu_{2}, p_{2}\right)
\end{array}\right) & g_{31}=\left(\begin{array}{c}
\left(\mu_{3}, p_{3}\right) \rightarrow\left(\mu_{1}, p_{1}\right) \\
\left(\mu_{1}, p_{1}\right) \rightarrow\left(\mu_{3}, p_{3}\right)
\end{array}\right)
\end{array}
$$




\begin{tabular}{|c|c|}
\hline $001 \equiv g_{\mu_{1} \mu_{2}} p_{1 \mu_{3}}$ & $002 \equiv g_{\mu_{1} \mu_{2}} p_{2 \mu_{3}}$ \\
\hline $200 \equiv g_{\mu_{2} \mu_{3}} p_{2 \mu_{1}}$ & $300 \equiv g_{\mu_{2} \mu_{3}} p_{3 \mu_{1}}$ \\
\hline $030 \equiv g_{\mu_{3} \mu_{1}} p_{3 \mu_{2}}$ & $010 \equiv g_{\mu_{3} \mu_{1}} p_{1 \mu_{2}}$ \\
\hline $211 \equiv p_{2 \mu_{1}} p_{1 \mu_{2}} p_{1 \mu_{3}}$ & $212 \equiv p_{2 \mu_{1}} p_{1 \mu_{2}} p_{2 \mu_{3}}$ \\
\hline $232 \equiv p_{2 \mu_{1}} p_{3 \mu_{2}} p_{2 \mu_{3}}$ & $332 \equiv p_{3 \mu_{1}} p_{3 \mu_{2}} p_{2 \mu_{3}}$ \\
\hline $331 \equiv p_{3 \mu_{1}} p_{3 \mu_{2}} p_{1 \mu_{3}}$ & $311 \equiv p_{3 \mu_{1}} p_{1 \mu_{2}} p_{1 \mu_{3}}$ \\
\hline $312 \equiv p_{3 \mu_{1}} p_{1 \mu_{2}} p_{2 \mu_{3}}$ & $231 \equiv p_{2 \mu_{1}} p_{3 \mu_{2}} p_{1 \mu_{3}}$ \\
\hline
\end{tabular}

Table 5.1: Definition of tensor abbreviations

and $g_{321}=g_{123}^{-1}$ one finds that $\left(g_{123}, g_{321}, g_{12}, g_{23}, g_{31}\right)$ yields $(+,+,-,-,-)$ when acting on $\Gamma_{\mu_{1} \mu_{2} \mu_{3}}\left(p_{1}, p_{2}, p_{3}\right)$.

The nonabelian nature of the permutation group $\mathcal{S}_{3}$ prevents one from finding a basis in which all of the tensors are eigenstates of all of these operators. Thus, aesthetic and physical principles must guide us in choosing convenient bases, two of which are discussed momentarily.

\subsubsection{Two Convenient Bases}

Let us consider the most general tensor structure. The three-index Lorentz-covariant tensor must be constructed out of the metric $g_{\mu \nu}$ and the momenta $\left(p_{i}^{\mu}\right)$. Since the momenta are not independent, $p_{1}+p_{2}+p_{3}=0$, simple combinatorics implies that there are in general 14 independent tensor components, 6 of which have one power of momenta and also the metric, and 8 of which have 3 powers of momenta. Many different basis choices can be made, although we will use essentially two.

In the subsequent discussion, some efficient notation will prove useful. This is summarized in Table 5.1.

Thus, each tensor is rewritten as a 3-slot object, where slots correspond to $\mu_{1}, \mu_{2}, \mu_{3}$ in that order, and the content of each slot is either ' 1 ' ' 2 ', or ' 3 ' to represent momentum $p_{1}, p_{2}, p_{3}$, or a ' 0 ', which must occur in pairs and represents that those two indices are connected by the metric tensor.

The most naive thing to do would be to just eliminate one momenta, say $p_{3}=$ $-p_{1}-p_{2}$ and use the following 14 basis tensors : 100, 200, 010, 020, 001, 002, 111, 112 , 
$121,211,122,212,221,222$. This is not very useful since the explicit Bose symmetry between the three gluons has been broken, and thus delicate relations between the form factors will have to enforce it.

The \pm Basis A more natural choice is obtained by starting from a manifestly symmetric, but redundant basis, which has 36 possible basis tensors, 9 with one power of momenta, and 27 with three powers of momenta. As a step towards our final basis, we find it convenient to eliminate all such tensors with momenta $p_{1}^{\mu_{1}}, p_{2}^{\mu_{2}}$, or $p_{3}^{\mu_{3}}$, i.e. anything with 1 in the first slot, 2 in second slot or 3 in the third slot. This yields the 14 basis tensors 001, 002, 200, 300, 030, 010, 211, 212, 232, 332, 331, 311, 231,312 , which are shown in Table 5.1. Note that under the action of $g_{123}$ we have $200 \rightarrow 030,300 \rightarrow 010,211 \rightarrow 232,311 \rightarrow 212$, etc. Also, notice that 200 and 300 are interchanged by the action of $g_{23}$, while 211 and 212 are interchanged by the action of $g_{12}$, etc. Thus, it is convenient to take appropriate linear combinations such that one of these interchange operators is diagonal for each tensor. Such basis tensors are

$$
\begin{aligned}
\hat{a}_{12} & =(00-)=001-002, \quad \hat{a}_{23}=(-00)=200-300, \quad \hat{a}_{31}=(0-0)=030-010 \\
\hat{b}_{12} & =(00+)=001+002, \quad \hat{b}_{23}=(+00)=200+300, \quad \hat{b}_{31}=(0+0)=030+010 \\
\hat{c}_{12} & =(++-), \quad \hat{c}_{23}=(-++), \quad \hat{c}_{31}=(+-+) \\
\hat{d}_{12} & =(--+), \quad \hat{d}_{23}=(+--), \quad \hat{d}_{31}=(-+-) \\
\hat{h} & =(---), \quad \hat{s}=(+++),
\end{aligned}
$$

where the notation means $( \pm \pm \pm) \equiv(2 \pm 3,3 \pm 1,1 \pm 2)$, so that $(++-)=231-$ $232+211-212+331-332+311-312$, etc. The subscripts are chosen because, for example, $\hat{a}_{12}$ is an eigenstate of $g_{12}$, etc.

Suppressing indices and momentum dependence, the three-gluon vertex is then written as

$$
\Gamma=\left(A_{12} \hat{a}_{12}+B_{12} \hat{b}_{12}+C_{12} \hat{c}_{12}+D_{12} \hat{d}_{12}+\text { perms }\right)+S \hat{s}+H \hat{h}
$$

where the lower case letters represent the basis tensors, while the upper case letters 
are the form factors, which depend on $p_{1}^{2}, p_{2}^{2}$, and $p_{3}^{2}$. In addition to indicating which basis tensors they are associated with, the subscripts on form factors also indicate the ordering of momenta in the arguments. For example, $A_{12}=A\left(p_{1}^{2}, p_{2}^{2} \mid p_{3}^{2}\right), A_{23}=$ $A\left(p_{2}^{2}, p_{3}^{2} \mid p_{1}^{2}\right), A_{31}=A\left(p_{3}^{2}, p_{1}^{2} \mid p_{2}^{2}\right)$, and the first two arguments are either symmetric or antisymmetric. The behavior of these form factors under $\mathcal{S}_{3}$ can be inferred from the behavior of the basis tensors under $\mathcal{S}_{3}$ (which will be discussed momentarily), along with the overall requirement for the vertex given below Eq. 5.6. One finds that $A(x, y \mid z)=+A(y, x \mid z)$, and thus $A_{12}=A_{21}$, etc. Similarly, $B_{12}=-B_{21}, C_{12}=C_{21}$, and $D_{12}=-D_{21} . H$ is totally invariant under the interchange or permutation of any momenta, while $S$ goes to $-S$ under any interchange of momenta, but is invariant under a cyclic permutation $g_{123}$.

It is straightforward to see that under the action of the permutation operator $\left(g_{123}\right)$ these fourteen basis tensors are organized into four triplets, $\left\{\hat{a}_{12}, \hat{a}_{23}, \hat{a}_{31}\right\}$, $\left\{\hat{b}_{12}, \hat{b}_{23}, \hat{b}_{31}\right\},\left\{\hat{c}_{12}, \hat{c}_{23}, \hat{c}_{31}\right\},\left\{\hat{d}_{12}, \hat{d}_{23}, \hat{d}_{31}\right\}$, as well as $\hat{h}$ and $\hat{s}$. The latter two are eigenstates of all five operators.

Consider the properties of $\left\{\hat{a}_{12}, \hat{a}_{23}, \hat{a}_{31}\right\}$ under the permutation group. It is easy to see that under the action of any element $g_{i}$, we have

$$
\left(\begin{array}{l}
\hat{a}_{12} \\
\hat{a}_{23} \\
\hat{a}_{31}
\end{array}\right) \rightarrow g_{i}\left(\begin{array}{c}
\hat{a}_{12} \\
\hat{a}_{23} \\
\hat{a}_{31}
\end{array}\right),
$$

with the matrices given by

$$
\begin{array}{ll}
g_{123}=\left(\begin{array}{lll}
0 & 1 & 0 \\
0 & 0 & 1 \\
1 & 0 & 0
\end{array}\right) & g_{321}=\left(\begin{array}{lll}
0 & 0 & 1 \\
1 & 0 & 0 \\
0 & 1 & 0
\end{array}\right) \\
g_{12}=-\left(\begin{array}{lll}
1 & 0 & 0 \\
0 & 0 & 1 \\
0 & 1 & 0
\end{array}\right) \quad g_{23}=-\left(\begin{array}{lll}
0 & 0 & 1 \\
0 & 1 & 0 \\
1 & 0 & 0
\end{array}\right) & g_{31}=-\left(\begin{array}{lll}
0 & 1 & 0 \\
1 & 0 & 0 \\
0 & 0 & 1
\end{array}\right)
\end{array}
$$

The transformation rules are identical for $\left\{c_{i j}\right\}$, and similar for $\left\{b_{i j}\right\}$ and $\left\{d_{i j}\right\}$ with 
the only change being that there is no minus sign in the three interchange operators $g_{12}, g_{23}$ and $g_{31}$.

The basis constructed above (Eq.(5.7)) will be called the \pm basis. As discussed later, this basis is the most convenient for phenomenology and furthermore the form factors exhibit particularly simple relations between the gluon, quark, and scalar contributions (Eqs. $(5.45,5.55))$.

However, the \pm basis as it stands does not contain the tree-level tensor structure. Thus, one is naturally led to diagonalizing the permutation operator $g_{123} .{ }^{1}$ Clearly, this is the most symmetric choice and, more importantly, one of the resulting eigenvectors is the tree level tensor structure.

In the triplet representation of $\mathcal{S}_{3}, g_{123}$ is diagonalized by the similarity transformation

$$
S=\frac{1}{\sqrt{3}}\left(\begin{array}{ccc}
1 & 1 & 1 \\
1 & \lambda & \bar{\lambda} \\
1 & \bar{\lambda} & \lambda
\end{array}\right) \quad S^{-1} g_{123} S \equiv \widetilde{g}_{123}=\left(\begin{array}{ccc}
1 & 0 & 0 \\
0 & \lambda & 0 \\
0 & 0 & \bar{\lambda}
\end{array}\right)
$$

where $\lambda=\exp \left(\frac{2 i \pi}{3}\right)=-\frac{1}{2}+i \frac{\sqrt{3}}{2}, \bar{\lambda}=\lambda^{*}$ are cube roots of unity. This results in new basis tensors and form factors

$$
\begin{aligned}
& \left(\begin{array}{l}
\hat{a}_{0} \\
\hat{a}_{+} \\
\hat{a}_{-}
\end{array}\right) \equiv \sqrt{3} S^{-1}\left(\begin{array}{l}
\hat{a}_{12} \\
\hat{a}_{23} \\
\hat{a}_{31}
\end{array}\right)=\left(\begin{array}{ccc}
1 & 1 & 1 \\
1 & \bar{\lambda} & \lambda \\
1 & \lambda & \bar{\lambda}
\end{array}\right)\left(\begin{array}{l}
\hat{a}_{12} \\
\hat{a}_{23} \\
\hat{a}_{31}
\end{array}\right) \\
& \left(\begin{array}{l}
A_{0} \\
A_{+} \\
A_{-}
\end{array}\right) \equiv \frac{1}{\sqrt{3}} S\left(\begin{array}{l}
A_{12} \\
A_{23} \\
A_{31}
\end{array}\right)=\frac{1}{3}\left(\begin{array}{lll}
1 & 1 & 1 \\
1 & \lambda & \bar{\lambda} \\
1 & \bar{\lambda} & \lambda
\end{array}\right)\left(\begin{array}{l}
A_{12} \\
A_{23} \\
A_{31}
\end{array}\right) .
\end{aligned}
$$

This procedure is repeated identically for the $(b, B),(c, C)$, and $(d, D)$ basis tensors and form factors.

Notice that $\hat{a}_{0}=g_{\mu_{1} \mu_{2}}\left(p_{1}-p_{2}\right)_{\mu_{3}}+g_{\mu_{2} \mu_{3}}\left(p_{2}-p_{3}\right)_{\mu_{1}}+g_{\mu_{3} \mu_{1}}\left(p_{3}-p_{1}\right)_{\mu_{2}}$ is the tree

\footnotetext{
${ }^{1}$ One can readily check that the only two operators in $\mathcal{S}_{3}$ which commute are $g_{123}$ and its inverse $g_{321}$. Thus one can diagonalize these two, OR one of the interchange operators $g_{12}, g_{23}, g_{31}$.
} 
level tensor, which is why the extra factors of $\sqrt{3}$ were included above.

The transformation properties for the basis tensors $\hat{a}_{i}$ and $\hat{c}_{i}$ are deduced from $\widetilde{g}_{123}$ given above, $\widetilde{g}_{321}=\operatorname{diag}(1, \bar{\lambda}, \lambda)$, and

$$
\widetilde{g}_{12}=-\left(\begin{array}{ccc}
1 & 0 & 0 \\
0 & 0 & 1 \\
0 & 1 & 0
\end{array}\right) \quad \widetilde{g}_{23}=-\left(\begin{array}{ccc}
1 & 0 & 0 \\
0 & 0 & \lambda \\
0 & \bar{\lambda} & 0
\end{array}\right) \quad \widetilde{g}_{31}=-\left(\begin{array}{ccc}
1 & 0 & 0 \\
0 & 0 & \bar{\lambda} \\
0 & \lambda & 0
\end{array}\right),
$$

while the transformation properties of the form factors are deduced by demanding that behavior given below Eq.(5.6) is respected. For example, since $\hat{a}_{-} \rightarrow-\bar{\lambda} \hat{a}_{+}$ under $\widetilde{g}_{23}$, we find $A_{-} \rightarrow \lambda A_{+}$so that $A_{-} \hat{a}_{-} \rightarrow-A_{-} \hat{a}_{-}$. For $(b, B)$ and $(d, D)$, the only change in the above is that there is not a minus sign in $\widetilde{g}_{12}, \widetilde{g}_{23}$, and $\widetilde{g}_{31}$.

We have not touched $\hat{h}$ and $\hat{s}$, as these are already eigenstates of all five operators $\left(g_{123}, g_{321}, g_{12}, g_{23}, g_{31}\right)$, with eigenvalues $(++---)$ and $(+++++)$, respectively.

We will call the above constructed basis the symmetric \pm basis. Note that any basis can be symmetrized in the same manner by diagonalizing the permutation operator $g_{123}$. The basis we started with in Eq.(5.7) is motivated by (a) its simple and symmetric construction from only metrics and $\left(p_{i+1} \pm p_{i-1}\right)_{\mu_{i}}$, (b) it is the most convenient basis for perturbative calculations, as will be discussed in section 4 , and (c) The individual form factors have a relatively simple form, as will be discussed in section 3 .

The LT Basis For some theoretical studies, another convenient basis is determined by the distinction between transverse $(T)$ and longitudinal $(L)$ tensors [80][81]. The $L$ tensors contribute to the Ward ID (or the more complicated SlavnovTaylor ID for the gauge-dependent vertex) while the $T$ tensors satisfy homogeneous equations $p_{3}^{\mu_{3}} \Gamma_{\mu_{1} \mu_{2} \mu_{3}}^{(T)}=0$. This is a very convenient basis for evaluating the loop corrections to the vertex, since the $L$ and $T$ parts separate, as described in Appendix A.

The \pm basis and the $L T$ basis are complementary in the following sense. The \pm basis is constructed out of combinations of longitudinal $(+)$ and transverse $(-)$ momenta, so that for example $(00+)=g_{\mu_{1} \mu_{2}}\left(p_{1 \mu_{3}}+p_{2 \mu_{3}}\right)=-g_{\mu_{1} \mu_{2}} p_{3 \mu_{3}}$ vanishes if the 
$\mu_{3}$ index is contracted into a conserved current. Meanwhile, the $L T$ basis distinguishes between parts of the vertex that do $(T)$ and do not $(L)$ vanish when dotted with longitudinal momenta. These straightforward relations to current conservation and Ward identities are essentially the reason these two bases are the most convenient to work with.

In our notation, the vertex can be written in the $L T$ basis as $\Gamma=\Gamma_{L}+\Gamma_{T}$, where

$$
\begin{aligned}
\Gamma_{L} & =\left(\bar{A}_{12} \bar{a}_{12}+\bar{B}_{12} \bar{b}_{12}+\bar{C}_{12} \bar{c}_{12}+\text { perms }\right)+\bar{S} \bar{s} \\
\Gamma_{T} & =\left(\bar{F}_{12} \bar{f}_{12}+\text { perms }\right)+\overline{H h},
\end{aligned}
$$

and the bar distinguishes this $L T$ basis from the \pm basis defined above in Eq.(5.7). The relation between basis tensors is given by

$$
\begin{aligned}
\bar{a}_{12} & =001-002=\hat{a}_{12} \\
\bar{b}_{12} & =001+002=\hat{b}_{12} \\
\bar{c}_{12} & =211-212-\left(p_{1} \cdot p_{2}\right)(001-002)=\frac{1}{4}\left(\hat{c}_{12}-\hat{d}_{23}+\hat{d}_{31}-\hat{h}\right)-\left(p_{1} \cdot p_{2}\right) \hat{a}_{12} \\
\bar{f}_{12} & =\left(p_{1} \cdot p_{2}\right)\left(\left(p_{2} \cdot p_{3}\right) 001-\left(p_{3} \cdot p_{1}\right) 002\right)-\left(\left(p_{2} \cdot p_{3}\right) 211-\left(p_{3} \cdot p_{1}\right) 212\right) \\
& =\frac{\left(p_{1} \cdot p_{2}\right)}{2}\left(-p_{3}^{2} \hat{a}_{12}+\left(p_{1}^{2}-p_{2}^{2}\right) \hat{b}_{12}\right) \\
& +\frac{1}{8}\left(p_{3}^{2}\left(\hat{c}_{12}-\hat{d}_{23}+\hat{d}_{31}-\hat{h}\right)+\left(p_{1}^{2}-p_{2}^{2}\right)\left(\hat{d}_{12}-\hat{c}_{23}+\hat{c}_{31}-\hat{s}\right)\right) \\
\bar{h} & =231-312-\left(\left(p_{1} \cdot p_{2}\right)(030-300)+\left(p_{2} \cdot p_{3}\right)(001-010)+\left(p_{3} \cdot p_{1}\right)(200-002)\right) \\
& =\frac{1}{4}\left(\hat{h}+\hat{c}_{12}+\hat{c}_{23}+\hat{c}_{31}\right)+\frac{1}{2}\left(p_{3}^{2} \hat{a}_{12}-\left(p_{1}^{2}-p_{2}^{2}\right) \hat{b}_{12}+p_{1}^{2} \hat{a}_{23}-\left(p_{2}^{2}-p_{3}^{2}\right) \hat{b}_{23}\right. \\
& \left.+p_{2}^{2} \hat{a}_{31}-\left(p_{3}^{2}-p_{1}^{2}\right) \hat{b}_{31}\right) \\
\bar{s} & =231+312=\frac{1}{4}\left(\hat{s}+\hat{d}_{12}+\hat{d}_{23}+\hat{d}_{31}\right),
\end{aligned}
$$


and we used $\left(p_{1} \cdot p_{2}\right)=\left(p_{3}^{2}-p_{1}^{2}-p_{2}^{2}\right) / 2$. This implies the relation between form factors

$$
\begin{aligned}
A_{12} & =\bar{A}_{12}-\left(p_{1} \cdot p_{2}\right) \bar{C}_{12}-\frac{p_{3}^{2}}{2}\left(\left(p_{1} \cdot p_{2}\right) \bar{F}_{12}-\bar{H}\right) \\
B_{12} & =\bar{B}_{12}+\frac{p_{1}^{2}-p_{2}^{2}}{2}\left(\left(p_{1} \cdot p_{2}\right) \bar{F}_{12}-\bar{H}\right) \\
C_{12} & =\frac{1}{4}\left(\bar{H}+\bar{C}_{12}+\frac{p_{3}^{2}}{2} \bar{F}_{12}+\frac{p_{1}^{2}-p_{3}^{2}}{2} \bar{F}_{31}+\frac{p_{2}^{2}-p_{3}^{2}}{2} \bar{F}_{23}\right) \\
D_{12} & =\frac{1}{4}\left(\bar{S}+\bar{C}_{23}-\bar{C}_{31}+\frac{p_{1}^{2}}{2} \bar{F}_{23}-\frac{p_{2}^{2}}{2} \bar{F}_{31}+\frac{p_{1}^{2}-p_{2}^{2}}{2} \bar{F}_{12}\right) \\
H & =\frac{1}{4}\left(\bar{H}-\bar{C}_{12}-\frac{p_{3}^{2}}{2} \bar{F}_{12}-\bar{C}_{23}-\frac{p_{1}^{2}}{2} \bar{F}_{23}-\bar{C}_{31}-\frac{p_{2}^{2}}{2} \bar{F}_{31}\right) \\
S & =\frac{1}{4}\left(\bar{S}+\frac{p_{2}^{2}-p_{1}^{2}}{2} \bar{F}_{12}+\frac{p_{3}^{2}-p_{2}^{2}}{2} \bar{F}_{23}+\frac{p_{1}^{2}-p_{3}^{2}}{2} \bar{F}_{31}\right),
\end{aligned}
$$

The unwritten form factors $\left(A_{23}\right.$, etc.) and basis tensors can be obtained trivially from the above equations by cyclic permutation $\left(g_{123}\right)$. In doing so it is useful to keep in mind the properties described under Eq.(5.8), along with $\bar{F}_{i j}=\bar{F}_{j i}, \bar{A}_{i j}=\bar{A}_{j i}$, $\bar{B}_{i j}=-\bar{B}_{j i}, \bar{C}_{i j}=\bar{C}_{j i}$, while $\bar{H}$ and $\bar{S}$ have the same transformation properties as $H$ and $S$, respectively.

\subsection{Results for the Form Factors}

In this section, we will present the results for the form factors in arbitrary dimension $d$ using dimensional regularization (DREG), for gluons in the adjoint representation, and massless quarks and scalars in arbitrary representations. The corrections due to supersymmetric regularization and massive fermions, scalars, and gauge bosons are given in detail in Appendices D and E, respectively.

The gauge-invariant vertex at one loop can be written as

$$
\begin{aligned}
& g \Gamma_{\mu_{1} \mu_{2} \mu_{3}}^{a b c}\left(p_{1}, p_{2}, p_{3}\right)=g f^{a b c}\left[\Gamma_{\mu_{1} \mu_{2} \mu_{3}}^{(0)}\left(p_{1}, p_{2}, p_{3}\right)\right. \\
& \left.-\frac{i g^{2}}{2}\left(C_{A} G_{\mu_{1} \mu_{2} \mu_{3}}+2 \sum_{f} T_{f} N_{f} Q_{\mu_{1} \mu_{2} \mu_{3}}+2 \sum_{s} T_{s} N_{s} S_{\mu_{1} \mu_{2} \mu_{3}}\right)\right]
\end{aligned}
$$


where the gluon $(G)$, quark $(Q)$, and scalar $(S)$ integrals are

$$
\begin{aligned}
G_{\mu_{1} \mu_{2} \mu_{3}} & =\int \frac{d^{d} l}{(2 \pi)^{d}} \frac{1}{l_{1}^{2} l_{2}^{2} l_{3}^{2}}\left(\Gamma_{\beta \mu_{1} \gamma}^{F}\left(l_{2}, p_{1},-l_{3}\right) \Gamma_{\gamma \mu_{2} \alpha}^{F}\left(l_{3}, p_{2},-l_{1}\right) \Gamma_{\alpha \mu_{3} \beta}^{F}\left(l_{1}, p_{3},-l_{2}\right)\right. \\
& +2\left(l_{2}+l_{3}\right)_{\mu_{1}}\left(l_{3}+l_{1}\right)_{\mu_{2}}\left(l_{1}+l_{2}\right)_{\mu_{3}}-8 l_{1}^{2}\left(g_{\mu_{1} \mu_{2}} p_{1 \mu_{3}}-g_{\mu_{1} \mu_{3}} p_{1 \mu_{2}}\right) \\
& \left.-8 l_{2}^{2}\left(g_{\mu_{2} \mu_{3}} p_{2 \mu_{1}}-g_{\mu_{2} \mu_{1}} p_{2 \mu_{3}}\right)-8 l_{3}^{2}\left(g_{\mu_{3} \mu_{1}} p_{3 \mu_{2}}-g_{\mu_{3} \mu_{2}} p_{3 \mu_{1}}\right)\right) \\
Q_{\mu_{1} \mu_{2} \mu_{3}} & =\int \frac{d^{d} l}{(2 \pi)^{d}} \frac{1}{l_{1}^{2} l_{2}^{2} l_{3}^{2}} \operatorname{Tr}\left[\gamma_{\mu_{1}} l_{3} \gamma_{\mu_{2}} l_{1} \gamma_{\mu_{3}} l_{2}\right] \\
S_{\mu_{1} \mu_{2} \mu_{3}} & =-\int \frac{d^{d} l}{(2 \pi)^{d}} \frac{1}{l_{1}^{2} l_{2}^{2} l_{3}^{2}}\left(l_{2}+l_{3}\right)_{\mu_{1}}\left(l_{3}+l_{1}\right)_{\mu_{2}}\left(l_{1}+l_{2}\right)_{\mu_{3}} .
\end{aligned}
$$

The gluon contribution was first derived in [60] using the pinch technique(PT), and is equivalent to the vertex obtained in the Background Field Method in quantum Feynman gauge(BFMFG). The quark and scalar integrals come straightforwardly from the one loop triangle diagrams. The notation and routing of the integral are defined in Fig.(5.1) such that $l_{1}=p_{2}+l_{3}, l_{2}=p_{3}+l_{1}, l_{3}=p_{1}+l_{2}$ and the tree level vertex $\Gamma^{(0)}$ and $\Gamma^{F}$ are defined as

$$
\begin{aligned}
\Gamma_{\mu_{1} \mu_{2} \mu_{3}}^{(0)}\left(p_{1}, p_{2}, p_{3}\right) & =g_{\mu_{1} \mu_{2}}\left(p_{1}-p_{2}\right)_{\mu_{3}}+g_{\mu_{2} \mu_{3}}\left(p_{2}-p_{3}\right)_{\mu_{1}}+g_{\mu_{3} \mu_{1}}\left(p_{3}-p_{1}\right)_{\mu_{2}} \\
\Gamma_{\beta \mu_{1} \gamma}^{F}\left(l_{2}, p_{1},-l_{3}\right) & =2 p_{1 \beta} g_{\mu_{1} \gamma}-2 p_{1 \gamma} g_{\mu_{1} \beta}-\left(l_{2}+l_{3}\right)_{\mu_{1}} g_{\beta \gamma} .
\end{aligned}
$$

All massless integrals can be reduced to two basic scalar integrals:

$$
\begin{aligned}
J & \equiv J\left(p_{1}^{2}, p_{2}^{2}, p_{3}^{2}\right)=\int \frac{d^{d} l}{(2 \pi)^{d}} \frac{1}{l_{1}^{2} l_{2}^{2} l_{3}^{2}} \\
J_{1} & \equiv J_{1}\left(p_{1}^{2}\right)=\int \frac{d^{d} l}{(2 \pi)^{d}} \frac{1}{l_{2}^{2} l_{3}^{2}}=\int \frac{d^{d} l}{(2 \pi)^{d}} \frac{1}{l^{2}\left(l+p_{1}\right)^{2}}
\end{aligned}
$$

These functions, and the massive integrals which are considered later, are summarized in Appendices A and B, where $J$ is written in terms of Clausen functions. In the following we will suppress the momentum arguments and write our results in terms of $J, J_{1}, J_{2}, J_{3}$. 


\subsection{1 (Supersymmetric) relations between gluons, quarks, and scalars}

Before presenting the results for individual form factors, which are somewhat lengthy, we will discuss the relationship between the gluon $(\mathrm{G})$, quark $(\mathrm{Q})$, and scalar $(\mathrm{S})$ contributions. We will end up finding relations similar to those found in the context of supersymmetric scattering amplitudes [72][73][82]. For a generic form factor $F$, let us write the one-loop contribution as

$$
F=i g^{2}\left(C_{A} F_{G}+2 \sum_{f} T_{f} N_{f} F_{Q}+2 \sum_{s} T_{s} N_{s} F_{S}\right)
$$

where the coupling constant $i g^{2}$ and group theory factors have been pulled out. The standard notation is used, so that $C_{A} \equiv C_{2}(G)=N_{c}$ for $S U\left(N_{c}\right)$, and $\operatorname{Tr}\left[t_{f}^{a} t_{f}^{b}\right]=$ $T_{f} \delta^{a b}$. Thus, $F_{Q}$ stands for the contribution of one Dirac fermion in the fundamental representation of $S U\left(N_{c}\right)$, or, due to a symmetry factor of $\frac{1}{2}$ for Weyl fermions, the contribution of adjoint gluinos divided by $N_{c}$. Similarly, $F_{S}$ stands for the contribution of one complex scalar in the fundamental representation, or the contribution of a real scalar in the adjoint, divided by $N_{c}$. These identifications will be used shortly.

After explicitly calculating the integrals in Eq.(5.18), we noticed that the gluon, quark, and scalar contributions have a similar structure for each form factor. To make this explicit, define the following sums for form factor $F$ :

$$
\begin{aligned}
\Sigma_{Q G}(F) & \equiv \frac{(d-2)}{2} F_{Q}+F_{G} \\
\Sigma_{S G}(F) & \equiv(d-2) F_{S}-F_{G} .
\end{aligned}
$$

Although the results for each form factor are often long, these sums are particularly simple, as can be seen in Eqs.(5.42,5.45,5.55). For all form factors in any basis, it also turns out that

$$
(d-10) \Sigma_{S G}=8 \Sigma_{Q G}
$$

and $\Sigma_{Q G}$ is always proportional to $d-10$. The above two equations and the results of Eqs. $(5.42,5.45,5.55)$ can be used to determine the $Q$ and $S$ contributions to any 
form factor, given the gluon contributions written explicitly below. Furthermore, Eqs. $(5.22,5.23)$ can be combined leading to

$$
F_{G}+4 F_{Q}+(10-d) F_{S}=0
$$

Considering the very different origins of each form factor (Eqs.(5.17,5.18)), it is remarkable that they are related in such a simple manner. Note that no such analogous relation holds for the gauge dependent vertex [81].

This type of relation hints at supersymmetry. To further understand the content of these relations, we will consider various supersymmetries in $d=4$.

- $\underline{\mathbf{N}=1}$ From the above definitions, it is clear that a vector superplet $V_{1}$ (gluons plus gluinos) contributes $i g^{2} N_{c}\left(F_{G}+F_{Q}\right) \equiv i g^{2} N_{c} F_{V_{1}}$ to a generic form factor $F$, while $N_{\Phi}$ chiral superplets contributes $i g^{2} N_{\Phi}\left(\frac{1}{2} F_{Q}+F_{S}\right) \equiv i g^{2} N_{\Phi} F_{\Phi}$. By the sum rule Eq. (5.24) in $d=4$, we have $F_{V_{1}}+6 F_{\Phi}=0$. Thus any form factor can be written

$$
F=i g^{2}\left(N_{c} F_{V_{1}}+N_{\Phi} F_{\Phi}\right)=\frac{i g^{2}}{3} \beta_{0}^{(N=1)} F_{V_{1}},
$$

where $\beta_{0}^{(N=1)}=3 N_{c}-\frac{1}{2} N_{\Phi}$ is the first $\beta$ function coefficient. Hence the contributions of vector and chiral superplets have precisely the same functional form for each form factor. Furthermore, every form factor is proportional to $\beta_{0}$ even though all but one of them are UV finite.

- $\underline{\mathbf{N}=\mathbf{2}}$ Here the vector superplet gives $i g^{2} N_{c}\left(F_{G}+2 F_{Q}+2 F_{S}\right) \equiv i g^{2} N_{c} F_{V_{2}}$, while $N_{h}$ hyperplets (a Weyl fermion of each helicity plus a doublet of complex scalars) yield $i g^{2} N_{h}\left(F_{Q}+2 F_{S}\right) \equiv i g^{2} N_{h} F_{h}$. The sum rule Eq.(5.24) can be written as $F_{V_{2}}+2 F_{h}=0$, and thus

$$
F=i g^{2}\left(N_{c} F_{V_{2}}+N_{h} F_{h}\right)=\frac{i g^{2}}{2} \beta_{0}^{(N=2)} F_{V_{2}},
$$

where $\beta_{0}^{(N=2)}=2 N_{c}-N_{h}$

- $\underline{\mathrm{N}=4}$ Here the vector superplet (the only multiplet allowed) contributes $2 i g^{2} N_{c}\left(F_{G}+\right.$ $\left.4 F_{Q}+6 F_{S}\right) \equiv N_{c} F_{V_{4}}$, which is identically zero by the sum rule, which of course 
is a consequence of $\beta_{0}^{(N=4)}=0$.

Thus, the similarities between form factors in $d=4$ are related to supersymmetric non-renormalization theorems. In particular, the exact conformal invariance of $N=4$ implies that the gauge-invariant three-gluon Green's function is not renormalized at any order in perturbation theory. Furthermore, at one-loop order there are not even finite corrections, as reflected in Eq.(5.24).

Analogous results hold for supersymmetry in $d \neq 4$. Here we must be careful, because in the sum rules and form factors presented in this chapter we worked in $d$ dimensions everywhere except in the traces over gamma matrices, where we used the conventional rule of dimensional regularization $\operatorname{tr}\left[\gamma_{\mu} \gamma_{\nu}\right]=4 g_{\mu \nu}$, and similarly for other traces. Properly working in integer-valued $d$ dimensions, we should use $\operatorname{tr}\left[\gamma_{\mu} \gamma_{\nu}\right]=d_{s}(d) g_{\mu \nu}$, where the spinor dimension of the gamma matrices is

$$
d_{s}(d)=\left(\begin{array}{c}
2^{d / 2} \\
2^{(d-1) / 2}
\end{array}\right) \text { for }\left(\begin{array}{c}
d \text { even } \\
d \text { odd }
\end{array}\right)
$$

Thus $F_{Q_{d}} \equiv \frac{d_{s}(d)}{4} F_{Q}$ is the contribution of a Dirac fermion in $d$ dimensions, and we have

$$
F_{G}+\frac{16}{d_{s}(d)} F_{Q_{d}}+(10-d) F_{S}=0
$$

Rather than using Eq.(5.28), one can alternatively use Eq.(5.24) and be sure to count fermion degrees of freedom in terms of $d=4$ spinors. Thus the Weyl fermions of $d=6$ and the Weyl-Majorana fermions of $d=10$ are composed of 2 and 4 Weyl fermions of four dimensions, respectively. From this, it is straightforward to show that $d=6, N=2$ and $d=10, N=1$ gauge theory give vanishing contribution to every form factor. For the $d=6, N=1$ case, one finds

$$
F=\frac{i g^{2}}{2} \beta_{0} F_{V_{1}} \quad \beta_{0}=2 N_{c}-N_{\Phi}
$$

where $\beta_{0}$ is determined from Eq.(5.37) in $d=6$.

Note that it is not straightforward to analytically continue $d_{s}(d)$ into arbitrary non-integer $d$, which is the reason for the simple dimensional regularization rule. 
However, the sum rule expressed in Eq.(5.24) is an analytic function of $d$ and thus represents an analytic continuation of supersymmetric non-renormalization theorems to arbitrary $d$. This is intimately related to the existence of a supersymmetry preserving regulator, dimensional reduction (DRED), where vector degrees of freedom are kept in four dimensions while the integrals are still performed in $d$ dimensions. Around four dimensions, $d=4-2 \epsilon$, we have $F_{G}+4 F_{Q}+(6+2 \epsilon) F_{S}=0$ in dimensional regularization, and we see that the $\epsilon$ term plays the role of the so-called $\epsilon$-ghosts of DRED. We have calculated the form factors in DRED (see Appendix D) and verified that

$$
F_{G}+4 F_{Q}+6 F_{S}=0 \quad \text { (DRED). }
$$

In the preceding discussion of supersymmetries in various dimensions we implicitly used DRED.

The extension of these relations to the massive case is outlined in Appendix E, where the full effects of internal massive fermions (MQ), massive scalars (MS), and massive gauge bosons (MG) are included, and the sum rule becomes

$$
F_{M G}+4 F_{M Q}+(9-d) F_{M S}=0
$$

in DREG while in DRED the only change is $9-d$ is replaced by 5 . Note that the external gluons remain massless and unbroken, so the internal massive gauge bosons might be the colored heavy gauge bosons arising in GUT models. The change of $10-d$ in the massless case to $9-d$ in the massive case reflects the fact that massive gauge bosons "eat" one scalar degree of freedom.

It should be emphasized that relations such as Eq.(5.24) do not exist for the gauge-dependent three-gluon vertex [81], since the gluon contributions depend on the gauge-parameter, while the quarks and scalars do not. Indeed, it is uniquely the pinch technique (or equivalently BFM in quantum Feynman gauge) Green's function which satisfies this homogeneous sum rule. For example, calculating in the BFM with $\xi_{Q} \neq 1$, leads to a nonzero RHS of Eq.(5.24). 
Since the sum rule applies to all form factors, one finds

$$
G_{\mu_{1} \mu_{2} \mu_{3}}+4 Q_{\mu_{1} \mu_{2} \mu_{3}}+(10-d) S_{\mu_{1} \mu_{2} \mu_{3}}=0
$$

which is remarkable given the expressions in Eq.(5.18). One can explicitly show this by performing the trace in Eq.(5.18) and some tedious algebra to rearrange the $\Gamma^{F} \Gamma^{F} \Gamma^{F}$ term. This can also be seen in the second order formalism of the BFM [72][73][82].

A similar relation holds for the one-loop gauge-invariant (pinch-technique) gluon two-point function in $d$ dimensions (the color factor $\delta^{a b}$ is suppressed),

$\Pi_{\mu_{1} \mu_{2}}(p)=\left(p^{2} g_{\mu_{1} \mu_{2}}-p_{\mu_{1}} p_{\mu_{2}}\right) \Pi\left(p^{2}\right) \equiv i g^{2}\left(N_{c} G_{\mu_{1} \mu_{2}}+2 \sum_{f} T_{f} N_{f} Q_{\mu_{1} \mu_{2}}+2 \sum_{s} T_{s} N_{s} S_{\mu_{1} \mu_{2}}\right)$,

where from Eqs. $(5.36,5.37)$ below we find

$$
G_{\mu_{1} \mu_{2}}+4 Q_{\mu_{1} \mu_{2}}+(10-d) S_{\mu_{1} \mu_{2}}=0
$$

Unfortunately, analogous relations do not hold for higher gauge-invariant gluon $n$-point functions. This is essentially because the color and spacetime indices mix nontrivially. However, inhomogeneous relations of the form

$$
G+4 Q+(10-d) S=\text { simple }
$$

still hold [72][73][82], where "simple" means an integral with fewer powers of loop momenta in the numerator. In the four-gluon case, this is just a simple scalar integral with no powers of loop momentum in the numerator. These loop-momentum counting rules have been derived in the second order formalism, which is reviewed in [82]. Note that the Ward ID for the four-gluon vertex [69] relates it to the three-gluon vertex, and thus the longitudinal parts of the four-gluon vertex must satisfy the homogeneous sum rules $F_{G}+4 F_{Q}+(10-d) F_{S}=0$.

It is interesting to see if extensions of these sum rules apply to two-loop calculations, where the supersymmetric Yukawa vertices must be taken into account. As a 
first application, the two-loop pinch technique gluon self-energy has been calculated including finite terms. Interestingly, the finite terms do not vanish for $N=4 \mathrm{SUSY}$, so it appears that the homogeneous sum rule in Eq.(5.24) does not have a counterpart at two loops. In any case, the finite parts of the two-loop result allow for an improved extraction of the PT couplings from data as well as giving the three loop beta function. This calculation is detailed in Chapter 6 .

Now explicit expressions for the form factors will be given, first in the $L T$ basis, and then in the \pm basis.

\subsubsection{The longitudinal form factors}

It is straightforward to solve the Ward identity (Eq.(5.5)) for the ten longitudinal form factors, defined in Eq.(5.14), in terms of the gluon self-energy function $\Pi$ defined by $\Pi_{\mu \nu}^{a b}(p)=\delta^{a b}\left(p^{2} g_{\mu \nu}-p_{\mu} p_{\nu}\right) \Pi\left(p^{2}\right)$. Note that this is not the usual gauge-dependent self-energy, but rather the gauge-invariant pinch technique self-energy. At one loop in $d$ dimensions this is given by

$$
\Pi\left(p^{2}\right)=i g^{2} \beta_{0}(d) \int \frac{d^{d} l}{(2 \pi)^{d}} \frac{1}{l^{2}(l+p)^{2}},
$$

where $\beta_{0}(d)$ is given by

$$
\beta_{0}(d)=\frac{7 d-6}{2(d-1)} C_{2}(G)-\frac{2(d-2)}{(d-1)} \sum_{f} T_{f} N_{f}-\frac{1}{(d-1)} \sum_{s} T_{s} N_{s}
$$

for massless gluons, quarks, and complex scalars. The mass-dependent results are given in section 5 and Appendix E. This result holds for dimensional regularization (DREG), whereas for dimensional reduction (DRED) the gluon coefficient changes from $(7 d-6)$ to $(8 d-10)$. 
The longitudinal form factors are given by

$$
\begin{aligned}
\bar{A}_{12} & =\frac{\Pi\left(p_{1}^{2}\right)+\Pi\left(p_{2}^{2}\right)}{2} \\
\bar{B}_{12} & =\frac{\Pi\left(p_{1}^{2}\right)-\Pi\left(p_{2}^{2}\right)}{2} \\
\bar{C}_{12} & =\frac{\Pi\left(p_{1}^{2}\right)-\Pi\left(p_{2}^{2}\right)}{p_{1}^{2}-p_{2}^{2}} \\
\bar{S} & =0,
\end{aligned}
$$

and of course cyclic permutations yield results for $\bar{A}_{23}$, etc. Note that one of the 14 form factors vanishes to all orders and only the $\bar{A}$ form factors contain UV divergences.

In the notation of Eqs. $(5.20,5.21)$ we have

$$
\begin{aligned}
& \bar{A}_{12}(G)=\frac{7 d-6}{2(d-1)} \frac{1}{2}\left(J_{1}+J_{2}\right) \\
& \bar{A}_{12}(Q)=\frac{2-d}{(d-1)} \frac{1}{2}\left(J_{1}+J_{2}\right) \\
& \bar{A}_{12}(S)=-\frac{1}{2(d-1)} \frac{1}{2}\left(J_{1}+J_{2}\right),
\end{aligned}
$$

and similarly for the $\bar{B}$ and $\bar{C}$ form factors.

\subsubsection{The transverse form factors}

These form factors cannot be determined from the Ward ID, and must be calculated explicitly. The algorithm used is briefly described in Appendix A.

Due to the lengthy expressions, the following shorthand notation will be used for the kinematic invariants:

$$
a=p_{1}^{2} \quad b=p_{2}^{2} \quad c=p_{3}^{2} \quad \alpha=p_{1} \cdot p_{2} \quad \beta=p_{2} \cdot p_{3} \quad \gamma=p_{3} \cdot p_{1}
$$


We also define the symmetric invariants

$$
\begin{aligned}
& \mathcal{Q}=\alpha+\beta+\gamma \\
& \mathcal{K}=\alpha \beta+\beta \gamma+\gamma \alpha \\
& \mathcal{P}=\alpha \beta \gamma .
\end{aligned}
$$

Note that the dot products can be written in terms of the virtualities $\alpha=(c-a-b) / 2$, $\beta=(a-b-c) / 2, \gamma=(b-c-a) / 2$, or vice versa $a=-\alpha-\gamma, b=-\alpha-\beta, c=-\beta-\gamma$, but the formulae are simpler and more transparent when selectively written in terms of both $\alpha, \beta, \gamma$ and $a, b, c$.

We will only write the full gluon contribution explicitly, since the quark and scalar parts can be determined from the results of section 3.1 (see Eqs. $(5.22,5.23,5.24)$ ) and the quark-gluon sum rules for the transverse form factors, which are

$$
\begin{aligned}
\Sigma_{Q G}\left(\bar{F}_{12}\right) & =-\frac{(d-10)}{2 \mathcal{K}}\left(\alpha J+\frac{2 \alpha\left(J_{1}-J_{2}\right)-\beta\left(J_{2}-J_{3}\right)-\gamma\left(J_{3}-J_{1}\right)}{\beta-\gamma}\right) \\
\Sigma_{Q G}(\bar{H}) & =\frac{(d-10)}{2} J
\end{aligned}
$$

The gluon contributions to the transverse form factors in the $L T$ basis are

$$
\begin{aligned}
\bar{F}_{12}(G) & =\frac{1}{2 \mathcal{K}^{2}}\left(J\left(10 \mathcal{P}+c\left(\mathcal{K}-7 \alpha^{2}-3 \beta \gamma\right)\right)\right. \\
& +\left(1-\frac{(d+1) \beta \gamma}{\mathcal{K}}\right)\left(\mathcal{P} J+\alpha \gamma J_{1}+\alpha \beta J_{2}+\beta \gamma J_{3}-\frac{\mathcal{K}}{d-1}\left(J_{1}+J_{2}+J_{3}\right)\right) \\
& +\frac{7 d-6}{2(d-1)(d-2)}\left[8 \mathcal{P}+(d-4) \alpha c^{2}+(d-2)(4 \mathcal{K} \alpha-c \beta \gamma)\right] \frac{J_{1}-J_{2}}{a-b} \\
& +\frac{1}{d-1}\left[2 \beta^{2}(d-2)-\frac{5 d-2}{2} \mathcal{K}-\frac{7 d-6}{d-2} \alpha \beta-\frac{2 d^{2}-15 d+14}{d-2} \alpha \gamma\right]\left(J_{2}-J_{3}\right) \\
& \left.-\frac{1}{d-1}\left[2 \gamma^{2}(d-2)-\frac{5 d-2}{2} \mathcal{K}-\frac{7 d-6}{d-2} \alpha \gamma-\frac{2 d^{2}-15 d+14}{d-2} \alpha \beta\right]\left(J_{3}-J_{1}\right)\right)
\end{aligned}
$$


and

$$
\begin{aligned}
\bar{H}(G) & =-\frac{1}{2 \mathcal{K}^{2}}\left(J\left[8 \mathcal{K}^{2}+(d-2) \mathcal{P} \mathcal{Q}+(d+1) \frac{a b c \mathcal{P}}{\mathcal{K}}\right]\right. \\
& +\frac{d-2}{d-1}\left[\alpha(\mathcal{K}-2 \alpha \gamma)\left(J_{1}-J_{2}\right)+\beta(\mathcal{K}-2 \beta \alpha)\left(J_{2}-J_{3}\right)+\gamma(\mathcal{K}-2 \beta \gamma)\left(J_{3}-J_{1}\right)\right] \\
& \left.+\mathcal{P} \frac{d+1}{d-1}\left[-\left(J_{1}+J_{2}+J_{3}\right)+\frac{d-1}{\mathcal{K}}\left(\alpha \gamma J_{1}+\alpha \beta J_{2}+\beta \gamma J_{3}\right)\right]\right)
\end{aligned}
$$

\subsubsection{The Form Factors in the Physical Basis}

Now we will present the results in the physical \pm basis (Eq.(5.7)), before symmetrization, Eq.(5.12), since this is the most convenient way to present the results. Of course, these results can be obtained from the relation between the \pm basis and the $L T$ basis given in Eq. (5.16), but we write them explicitly for future convenience and phenomenological applications.

The quark-gluon sums are given by

$$
\begin{aligned}
\Sigma_{Q G}\left(A_{12}\right) & =\frac{(d-10)}{4 \mathcal{K}}\left(a b c J+a \beta J_{1}+b \gamma J_{2}+c \alpha J_{3}\right) \\
\Sigma_{Q G}\left(B_{12}\right) & =\frac{(d-10)}{4 \mathcal{K}}\left((\gamma-\beta) a b J+(2 \alpha+\beta) a J_{1}-(2 \alpha+\gamma) b J_{2}-\alpha(\beta-\gamma) J_{3}\right) \\
\Sigma_{Q G}\left(C_{12}\right) & =-\frac{(d-10)}{4 \mathcal{K}}\left(\alpha c J+\gamma J_{1}+\beta J_{2}+c J_{3}\right) \\
\Sigma_{Q G}\left(D_{12}\right) & =0 \\
\Sigma_{Q G}(H) & =0 \\
\Sigma_{Q G}(S) & =0
\end{aligned}
$$

and the remaining sums (for $A_{23}$, etc.) are related trivially by permutations $\alpha \rightarrow$ $\beta \rightarrow \gamma \rightarrow \alpha, a \rightarrow b \rightarrow c \rightarrow a, J_{1} \rightarrow J_{2} \rightarrow J_{3} \rightarrow J_{1}$. 
The gluon form factors in $d$ dimensions are

$$
\begin{aligned}
& -4 \mathcal{K}^{2} A_{12}(G)=a b c J(7 \mathcal{K}+\beta \gamma)+a J_{1}\left(7 \mathcal{K} \beta+\beta^{2} \gamma+\mathcal{K} \gamma \frac{d-2}{d-1}\right) \\
& +\quad b J_{2}\left(7 \mathcal{K} \gamma+\beta \gamma^{2}+\mathcal{K} \beta \frac{d-2}{d-1}\right)+c J_{3}\left(7 \mathcal{K} \alpha+\mathcal{P}+\mathcal{K} c \frac{d-2}{d-1}\right) \\
& -4 \mathcal{K}^{2} B_{12}(G)=a b J(7 \mathcal{K}+\beta \gamma)(\gamma-\beta)+a J_{1}\left(7 \mathcal{K} \beta-b \gamma(\beta-\gamma)+\mathcal{K} \frac{2 \alpha(7 d-6)+\gamma}{d-1}\right) \\
& \quad-\quad b J_{2}\left(7 \mathcal{K} \gamma+a \beta(\beta-\gamma)+\mathcal{K} \frac{2 \alpha(7 d-6)+\beta}{d-1}\right)+(\gamma-\beta) J_{3}\left(7 \mathcal{K} \alpha+\mathcal{P}+\mathcal{K} c \frac{d-2}{d-1}\right) \\
& 16 \mathcal{K}^{3} C_{12}(G)=c J\left(3 \mathcal{K}^{2}(10 \alpha+c)+\mathcal{K}\left(\alpha^{3}-6 c \beta \gamma\right)+\mathcal{P} \mathcal{K}(d+4)-\mathcal{P}(d+1)\left(\alpha^{2}+2 \beta \gamma\right)\right) \\
& +\quad J_{1}\left(\mathcal{K}^{2}\left[\frac{(3-2 d)}{d-1} \alpha-\gamma \frac{\left(d^{2}-30 d+24\right)}{d-1}+3 \beta\right]+\mathcal{P}(d+1)\left[\frac{\mathcal{K}}{d-1}+\gamma(2 \mathcal{Q}-3 \alpha)\right]\right. \\
& \left.+\gamma \mathcal{K}\left[\frac{\left(d^{2}-3\right)}{d-1} \alpha^{2}-6 \beta^{2}+\frac{\left(d^{2}-8 d+9\right)}{d-1} \beta \gamma-4 \gamma^{2} \frac{(d-2)}{d-1}\right]\right) \\
& +\quad J_{2}\left(\mathcal{K}^{2}\left[\frac{(3-2 d)}{d-1} \alpha-\beta \frac{\left(d^{2}-30 d+24\right)}{d-1}+3 \gamma\right]+\mathcal{P}(d+1)\left[\frac{\mathcal{K}}{d-1}+\beta(2 \mathcal{Q}-3 \alpha)\right]\right. \\
& \left.+\beta \mathcal{K}\left[\frac{\left(d^{2}-3\right)}{d-1} \alpha^{2}-6 \gamma^{2}+\frac{\left(d^{2}-8 d+9\right)}{d-1} \beta \gamma-4 \beta^{2} \frac{(d-2)}{d-1}\right]\right) \\
& +c J_{3}\left(\frac{(30 d-31)}{d-1} \mathcal{K}^{2}+\mathcal{K}\left[\alpha^{2}-4 c^{2} \frac{(d-2)}{d-1}-\frac{\left(d^{2}-4 d+1\right)}{d-1} \beta \gamma\right]+(d+1) \mathcal{P}(2 \mathcal{Q}-3 \alpha)\right)
\end{aligned}
$$




$$
\begin{aligned}
& 16 \mathcal{K}^{3} D_{12}(G)=a b(a-b) J(\mathcal{K}(\mathcal{Q}+2 \alpha)-(d+1) \mathcal{P})-a J_{1}\left(\frac{d^{2}-4}{d-1} \mathcal{K}^{2}\right. \\
& \left.+\mathcal{K}\left[\beta^{2}-\beta \gamma \frac{\left(d^{2}-3\right)}{d-1}-\alpha \beta \frac{\left(d^{2}-4 d+1\right)}{d-1}+4 \alpha^{2} \frac{(d-2)}{d-1}\right]-(2 \alpha+\beta) \mathcal{P}(d+1)\right) \\
& +b J_{2}\left(\frac{\left(d^{2}-4\right)}{d-1} \mathcal{K}^{2}+\mathcal{K}\left[\gamma^{2}-\beta \gamma \frac{\left(d^{2}-3\right)}{d-1}-\alpha \gamma \frac{\left(d^{2}-4 d+1\right)}{d-1}+4 \alpha^{2} \frac{(d-2)}{d-1}\right]\right. \\
& -(2 \alpha+\gamma) \mathcal{P}(d+1))+(a-b) J_{3}\left(\frac{(4 d-7)}{d-1} \mathcal{K}^{2}+\mathcal{K}\left[3 \alpha^{2}-\beta \gamma \frac{\left(d^{2}-3\right)}{d-1}\right]-\alpha \mathcal{P}(d+1)\right) \\
& 16 \mathcal{K}^{3} H(G)=a b c J(\mathcal{P}(d+1)-\mathcal{K} \mathcal{Q}) \\
& +\quad a J_{1}\left(\frac{3-2 d}{d-1} \mathcal{K}^{2}+\left[\frac{d^{2}-3}{d-1} \alpha \gamma-\beta^{2}\right] \mathcal{K}+(d+1) \dot{\beta} \mathcal{P}\right) \\
& +\quad b J_{2}\left(\frac{3-2 d}{d-1} \mathcal{K}^{2}+\left[\frac{d^{2}-3}{d-1} \alpha \beta-\gamma^{2}\right] \mathcal{K}+(d+1) \gamma \mathcal{P}\right) \\
& +c J_{3}\left(\frac{3-2 d}{d-1} \mathcal{K}^{2}+\left[\frac{d^{2}-3}{d-1} \beta \gamma-\alpha^{2}\right] \mathcal{K}+(d+1) \alpha \mathcal{P}\right) \\
& 16 \mathcal{K}^{3} S(G)=(a-b)(b-c)(c-a) J(3 \mathcal{K} \mathcal{Q}-(d+1) \mathcal{P}) \\
& +(b-c) J_{1}\left(\frac{3 \mathcal{K}^{2}}{d-1}+\mathcal{K}\left[4 a^{2} \frac{d-2}{d-1}+\alpha \gamma \frac{d^{2}-4 d+1}{d-1}-3 \beta^{2}\right]-(d+1) \mathcal{P}(2 \mathcal{Q}-3 \beta)\right) \\
& +(c-a) J_{2}\left(\frac{3 \mathcal{K}^{2}}{d-1}+\mathcal{K}\left[4 b^{2} \frac{d-2}{d-1}+\alpha \beta \frac{d^{2}-4 d+1}{d-1}-3 \gamma^{2}\right]-(d+1) \mathcal{P}(2 \mathcal{Q}-3 \gamma)\right) \\
& +(a-b) J_{3}\left(\frac{3 \mathcal{K}^{2}}{d-1}+\mathcal{K}\left[4 c^{2} \frac{d-2}{d-1}+\beta \gamma \frac{d^{2}-4 d+1}{d-1}-3 \alpha^{2}\right]-(d+1) \mathcal{P}(2 \mathcal{Q}-3 \alpha)\right)
\end{aligned}
$$

Now we turn to the physical symmetrized basis. From Eq.(5.12), we see that for 
any triplet of form factors, say $A_{i j}$, we have

$$
\begin{aligned}
& A_{0}=\frac{1}{3}\left(A_{12}+A_{23}+A_{31}\right) \\
& A_{+}=\frac{1}{3}\left(A_{12}+\lambda A_{23}+\bar{\lambda} A_{31}\right) \equiv A_{1}+i A_{2} \\
& A_{-}=\frac{1}{3}\left(A_{12}+\bar{\lambda} A_{23}+\lambda A_{31}\right) \equiv A_{1}-i A_{2}
\end{aligned}
$$

where we have defined

$$
\begin{aligned}
& A_{1}=\frac{1}{3}\left(A_{12}-\frac{1}{2}\left(A_{23}+A_{31}\right)\right) \\
& A_{2}=\frac{\sqrt{3}}{6}\left(A_{23}-A_{31}\right) .
\end{aligned}
$$

$A_{1}$ and $A_{2}$ correspond to the real and imaginary parts of $A_{ \pm}$only when $J, J_{1}, J_{2}, J_{3}$ are real. This occurs (in the massless case) when $\mathcal{K}>0$, which can only happen if all three gluon virtualities are of the same sign, either all spacelike or all timelike. This is often not the case for real problems. In general, however, it can be shown that

$$
\begin{aligned}
A_{ \pm}^{*}(a, b, c) & =A_{\mp}(-a,-b,-c) \\
B_{0}^{*}(a, b, c) & =B_{0}(-a,-b,-c) \\
B_{ \pm}^{*}(a, b, c) & =B_{\mp}(-a,-b,-c) \\
C_{0}^{*}(a, b, c) & =-C_{0}(-a,-b,-c) \\
C_{ \pm}^{*}(a, b, c) & =-C_{\mp}(-a,-b,-c) \\
D_{0}^{*}(a, b, c) & =-D_{0}(-a,-b,-c) \\
D_{ \pm}^{*}(a, b, c) & =-D_{\mp}(-a,-b,-c) \\
H^{*}(a, b, c) & =-H(-a,-b,-c) \\
S^{*}(a, b, c) & =-S(-a,-b,-c) .
\end{aligned}
$$

Furthermore, all of the above form factors except for $A_{0}$ are scale invariant,

$$
F(\lambda a, \lambda b, \lambda c)=F(a, b, c)=F(a / c, b / c, 1) \quad \lambda>0
$$


Only $A_{0}$ is not scale invariant and does not satisfy a simple reality condition. The quark-gluon sums are given by

$$
\begin{aligned}
\Sigma_{Q G}\left(A_{0}\right) & =\frac{(d-10)}{4 \mathcal{K}} \Phi_{0} \quad \Sigma_{Q G}\left(A_{ \pm}\right)=0 \\
\Sigma_{Q G}\left(B_{0}\right) & =-\frac{(d-10)}{8 \mathcal{K}} B_{0}(G) \\
\Sigma_{Q G}\left(B_{ \pm}\right) & =\frac{(d-10)}{36 \mathcal{K}}\left(-3\left(\mathcal{Q} \Phi_{2}+\mathcal{K} J(\beta-\gamma)\right) \pm i \sqrt{3}\left(\mathcal{Q} \Phi_{1}-\mathcal{K} J(\mathcal{Q}-3 \alpha)\right)\right) \\
\Sigma_{Q G}\left(C_{0}\right) & =\frac{(d-10)}{6} J \\
\Sigma_{Q G}\left(C_{ \pm}\right) & =\frac{(d-10)}{24 \mathcal{K}}\left(\Phi_{1} \pm i \sqrt{3} \Phi_{2}\right) \\
\Sigma_{Q G}\left(D_{0}\right) & =\Sigma_{Q G}\left(D_{ \pm}\right)=0 \\
\Sigma_{Q G}(H) & =\Sigma_{Q G}(S)=0
\end{aligned}
$$

where we have defined the commonly occuring functions

$$
\begin{aligned}
& \Phi_{0}=a b c J+a \beta J_{1}+b \gamma J_{2}+c \alpha J_{3} \\
& \Phi_{1}=(\mathcal{K}-3 \beta \gamma) J-3 \gamma J_{1}-3 \beta J_{2}+3(\beta+\gamma) J_{3} \\
& \Phi_{2}=\alpha(\beta-\gamma) J+(2 \alpha+\gamma) J_{1}-(2 \alpha+\beta) J_{2}+(\beta-\gamma) J_{3} .
\end{aligned}
$$

From the definition of $\Sigma_{Q G}$ in Eq.(5.22) we see that the quark and gluon (and thus scalar, by Eq.(5.23)) contributions have the same functional form for the seven form factors which have a zero in the above. Letting $F$ stand for $A_{ \pm}, D_{0}, D_{ \pm}, H$ or $S$, we find that

$$
F=i g^{2}\left(N_{c}-\frac{4}{d-2} \sum_{f} T_{f} N_{f}+\frac{2}{d-2} \sum_{s} T_{s} N_{s}\right) F(G),
$$

which, in $d=4 \mathrm{QCD}$, reduces to $F=i g^{2}\left(N_{c}-N_{f}\right) F(G)$.

In addition, both $A_{0}$ and $B_{0}$ are governed by one function, since they satisfy 
different sum rules. In particular, the tree level tensor structure has coefficient

$$
A_{0}=-\frac{i g^{2}}{2 \mathcal{K}}\left(\frac{11}{3} C_{A}-\frac{2(3 d-8)}{3(d-2)} \sum_{f} T_{f} N_{f}-\frac{2}{3(d-2)} \sum_{s} T_{s} N_{s}\right) \Phi_{0} .
$$

This form factor will be discussed in more detail in the next section.

Also, one finds from explicit calculation that the scalar contribution to $B_{0}$ vanishes,

$$
B_{0}(S)=0 \quad B_{0}(G)+4 B_{0}(Q)=0
$$

and thus

$$
\begin{aligned}
B_{0} & =i g^{2}\left(N_{c}-\frac{1}{2} \sum_{f} T_{f} N_{f}\right) B_{0}(G) \quad \text { where } \\
B_{0}(G) & =-\frac{2}{3 \mathcal{K}}\left((a-b)(b-c)(c-a) J+(b-c)(2 \mathcal{Q}-3 \beta) J_{1}+(c-a)(2 \mathcal{Q}-3 \gamma) J_{2}\right. \\
& \left.+(a-b)(2 \mathcal{Q}-3 \alpha) J_{3}\right)
\end{aligned}
$$

Finally, since $\bar{S}=0$ exactly, we know that our fourteen-dimensional basis is degenerate, which is reflected in the fact that $S+3 D_{0}=0$. Hence we define a new basis tensor $\hat{d}_{0}^{\prime}=\hat{d}_{0}-3 \hat{s}$ so that $D_{0} \hat{d}_{0}+S \hat{s}=D_{0}\left(\hat{d}_{0}-3 \hat{s}\right)=D_{0} \hat{d}_{0}^{\prime}$.

Thus, we find that eight of the thirteen nonzero form factors have the same functional form for gluons, quarks, and scalars. Only the five form factors $B_{ \pm}, C_{0}$ and $C_{ \pm}$ do not. These statements are basis dependent. One can always find bases where none of the form factors have a vanishing $Q G$ sum rule. In the course of our calculations, we found that the \pm basis gives the maximum number of such zeroes among bases which are reasonable and contain the tree-level tensor structure. In this sense the (symmetric) \pm basis is the simplest and most compelling. We will see in the next section that this is also the most convenient basis for perturbative calculations. Of course, as discussed in a previous section, with supersymmetry every form factor is proportional to $\beta_{0}$, and so supermultiplets are governed by the same function in any basis. 


\subsection{Three-Gluon Vertex in Perturbation Theory}

Applying the pinch-technique (PT) construction to the three-gluon vertex occurring in a physical process involving three external on-shell legs, one arrives at a dressed tree-level skeleton graph, dressed with pinch-technique vertices and self-energies as shown in Fig. 5.2. Generically the amplitude of the three-gluon graph can be written

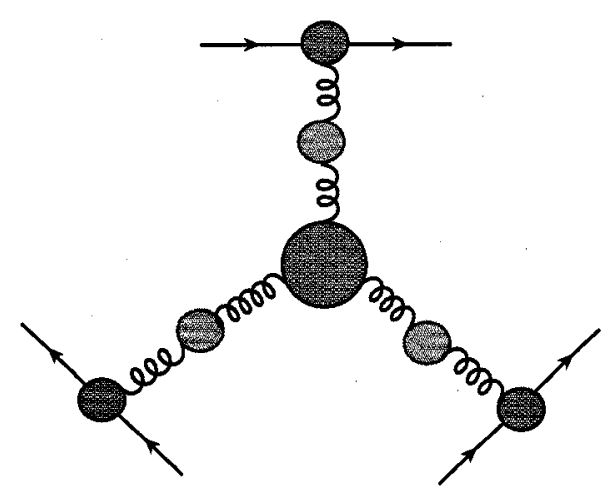

(A)

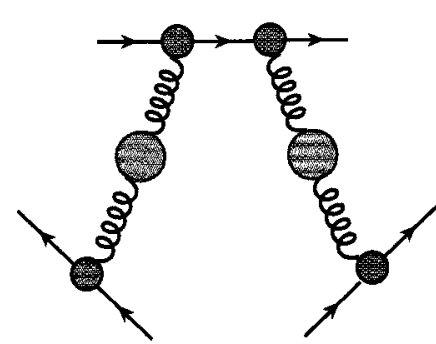

(B)

Figure 5.2: The tree-level skeleton graphs dressed with pinch-technique vertices and self-energies, which are used to define effective charges both for the quark-quark-gluon vertex (depending on a single gluon virtuality) and for the three-gluon vertex, which depends on three different momenta.

$$
\mathcal{M}=\mathcal{C} g_{0}^{4} V_{1}^{\nu_{1}} V_{2}^{\nu_{2}} V_{3}^{\nu_{3}} D_{\mu_{1} \nu_{1}}\left(k_{1}\right) D_{\mu_{2} \nu_{2}}\left(k_{2}\right) D_{\mu_{3} \nu_{3}}\left(k_{3}\right) \Gamma_{\mu_{1} \mu_{2} \mu_{3}}\left(k_{1}, k_{2}, k_{3}\right),
$$

where $\mathcal{C}$ is the overall color factor and $g_{0}$ is the bare coupling. The PT vertices $V_{i}$ are for gluons coupled to external particles, whose indices are suppressed; this is shown in Fig.5.2 for external quarks. $\Gamma=\sum_{i=1}^{13} F_{i} \hat{f}_{i}$ is the gauge invariant three gluon vertex, whose thirteen form factors $\left(F_{i}\right)$ are given in the preceding section. Finally the "gauge 
invariant" PT gluon propagator is

$$
\begin{aligned}
D_{\mu \nu}(k) & =\frac{1}{k^{2}}\left(\frac{t_{\mu \nu}(k)}{1+\Pi\left(k^{2}\right)}+\xi l_{\mu \nu}(k)\right) \\
t_{\mu \nu}(k) & =\left\{\begin{array}{c}
g_{\mu \nu}-\frac{k_{\mu} k_{\nu}}{k^{2}} \\
g_{\mu \nu}-\frac{n_{\mu} k_{\nu}+k_{\mu} n_{\nu}}{n \cdot k}
\end{array}\right\} \text { in }\left\{\begin{array}{c}
\text { covariant } \\
\text { axial }
\end{array}\right\} \text { gauges } \\
l_{\mu \nu}(k) & =\left\{\begin{array}{c}
\frac{k_{\mu} k_{\nu}}{k^{2}} \\
\frac{k_{\mu} k_{\nu}}{(n \cdot k)^{2}}
\end{array}\right\} \text { in }\left\{\begin{array}{c}
\text { covariant } \\
\text { axial }
\end{array}\right\} \text { gauges, }
\end{aligned}
$$

where $\xi$ is the gauge-fixing parameter. This is "gauge invariant" in the maximal sense, i.e. the gauge dependence comes only from the tree level terms, and in particular $\Pi\left(k^{2}\right)$ is totally gauge invariant.

Regardless of whether the external particles are quarks, gluons, or scalars, the vertices satisfy $k_{1}^{\mu} V_{1, \mu}=0$ when these particles are on shell (OS). One can then show that the gauge dependent terms coming from Eq.(5.62) vanish in the full amplitude consisting of all of the graphs in Fig.(5.2). This can be seen trivially in the covariant gauges where the gauge cancelations occur graph by graph, and with some work in axial gauges, where the cancelation occurs between all of the graphs. In the latter case, one must use the fact that the three gluon vertex satisfies the Ward ID in Eq.(5.3). Therefore we can take

$$
D_{\mu \nu}(k) \rightarrow \frac{g_{\mu \nu}}{k^{2}\left(1+\Pi\left(k^{2}\right)\right)}
$$

Also, in the \pm basis (Eq. (5.7)) any tensor with $\mathrm{a}^{\prime}+$ ' in any slot gives vanishing contribution to $\mathcal{M}$. For example, $(+00)=\left(k_{2}+k_{3}\right)_{\mu_{1}} g_{\mu_{2} \mu_{3}}=-k_{1, \mu_{1}} g_{\mu_{2} \mu_{3}}$, and $k_{1, \mu_{1}}$ dots into $V_{1, \mu_{1}}$ yielding zero. Hence only $(00-),(-00),(0-0)$, and (-- ) contribute, and we find

$$
\mathcal{M}=\frac{\mathcal{C} g_{0}^{3}}{\left(1+\Pi\left(k_{1}^{2}\right)\right)\left(1+\Pi\left(k_{2}^{2}\right)\right)\left(1+\Pi\left(k_{3}^{2}\right)\right)} \frac{V_{1}^{\mu_{1}} V_{2}^{\mu_{2}} V_{3}^{\mu_{3}}}{k_{1}^{2} k_{2}^{2} k_{3}^{2}} g_{0} \Gamma_{\mu_{1} \mu_{2} \mu_{3}}^{O S}\left(k_{1}, k_{2}, k_{3}\right),
$$


where the three-gluon vertex connected to on-shell (OS) external particles is

$$
\Gamma_{\mu_{1} \mu_{2} \mu_{3}}^{O S}\left(k_{1}, k_{2}, k_{3}\right)=\left(1+A_{0}\right) \hat{a}_{0}+A_{+} \hat{a}_{+}+A_{-} \hat{a}_{-}+H \hat{h}
$$

in the notation of section 5.2 where hatted objects are three index basis tensors.

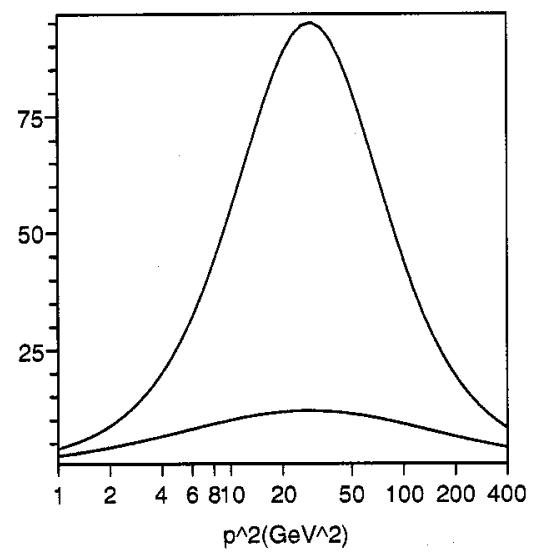

Figure 5.3: The effective scale $Q_{e f f}^{2}\left(10 \mathrm{GeV}^{2}, 10 \mathrm{GeV}^{2}, p^{2}\right)$ is the lower blue curve, while $Q_{e f f}^{2}\left(-10 \mathrm{GeV}^{2},-10 \mathrm{GeV}^{2}, p^{2}\right)=Q_{e f f}^{2}\left(10 \mathrm{GeV}^{2}, 10 \mathrm{GeV}^{2},-p^{2}\right)$ is the upper red curve. These both asymptote to zero, although very slowly for the upper curve.

Now one naturally defines PT effective charges by ${ }^{2}$

$$
g^{2}\left(k_{i}^{2}\right) \equiv \frac{g_{0}^{2}}{1+\Pi\left(k_{i}^{2}\right)} \quad i=1,2,3
$$

Since we only have a single power of $g_{0}$ for each $1 /\left(1+\Pi\left(k_{i}^{2}\right)\right)$, this leaves the factor $\prod_{i=1}^{3} 1 / \sqrt{1+\Pi\left(k_{i}^{2}\right)} \approx 1-\frac{1}{2}\left(\Pi\left(k_{1}^{2}\right)+\Pi\left(k_{2}^{2}\right)+\Pi\left(k_{3}^{2}\right)\right)$ to be absorbed into the three gluon vertex. Thus we have

$$
\begin{gathered}
\prod_{i=1}^{3} \frac{1}{\sqrt{1+\Pi\left(k_{i}^{2}\right)}} \Gamma_{\mu_{1} \mu_{2} \mu_{3}}^{O S}\left(k_{1}, k_{2}, k_{3}\right)=\left(1+\widetilde{A}_{0}\right) \hat{a}_{0}+A_{+} \hat{a}_{+}+A_{-} \hat{a}_{-}+H \hat{h} \\
\widetilde{A}_{0}\left(k_{1}^{2}, k_{2}^{2}, k_{3}^{2}\right) \equiv A_{0}\left(k_{1}^{2}, k_{2}^{2}, k_{3}^{2}\right)-\frac{1}{2}\left(\Pi\left(k_{1}^{2}\right)+\Pi\left(k_{2}^{2}\right)+\Pi\left(k_{3}^{2}\right)\right)
\end{gathered}
$$

\footnotetext{
${ }^{2} \mathrm{Eq} .(5.66)$ holds for external fermions or scalars, but for gluons one would instead have three additional three gluon effective couplings, as is clear from the derivation of Eq.(5.69).
} 


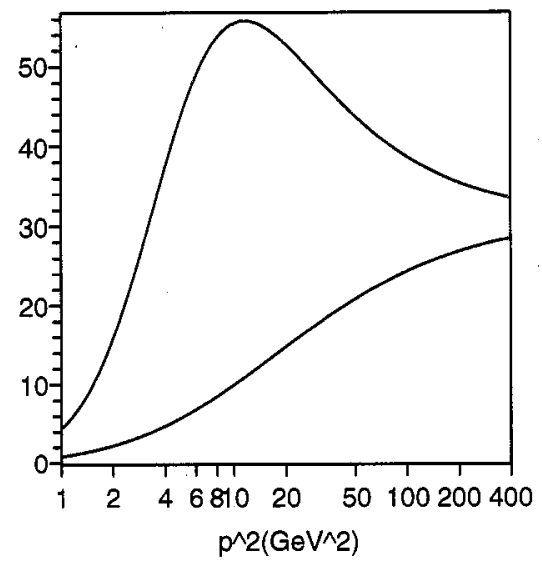

Figure 5.4: The effective scale $Q_{e f f}^{2}\left(10 \mathrm{GeV}^{2}, p^{2}, p^{2}\right)$ is the lower blue curve, while $Q_{e f f}^{2}\left(-10 \mathrm{GeV}^{2}, p^{2}, p^{2}\right)=Q_{e f f}^{2}\left(10 \mathrm{GeV}^{2},-p^{2},-p^{2}\right)$ is the upper red curve. These both asymptote to $10 e^{\Omega} \mathrm{GeV}^{2} \approx 31.25 \mathrm{GeV}^{2}$.

and

$$
\mathcal{M}=\mathcal{C} g\left(k_{1}^{2}\right) g\left(k_{2}^{2}\right) g\left(k_{3}^{2}\right) \frac{V_{1}^{\mu_{1}} V_{2}^{\mu_{2}} V_{3}^{\mu_{3}}}{k_{1}^{2} k_{2}^{2} k_{3}^{2}} g_{0}\left[\left(1+\widetilde{A}_{0}\right) \hat{a}_{0}+A_{+} \hat{a}_{+}+A_{-} \hat{a}_{-}+H \hat{h}\right] .
$$

This naturally leads to the effective coupling of the three-gluon vertex

$$
\begin{aligned}
\widetilde{g}\left(k_{1}^{2}, k_{2}^{2}, k_{3}^{2}\right) & \equiv g_{0}\left(1+\widetilde{A}_{0}\left(k_{1}^{2}, k_{2}^{2}, k_{3}^{2}\right)\right) \\
\widetilde{\alpha}(a, b, c) & \equiv \frac{\widetilde{g}^{2}(a, b, c)}{4 \pi} \approx \frac{\alpha_{0}}{1-2 \widetilde{A}_{0}(a, b, c)}
\end{aligned}
$$

first obtained by Lu in [83]. Our amplitude then takes the final form

$$
\mathcal{M}=\mathcal{C} g\left(k_{1}^{2}\right) g\left(k_{2}^{2}\right) g\left(k_{3}^{2}\right) \widetilde{g}\left(k_{1}^{2}, k_{2}^{2}, k_{3}^{2}\right) \frac{V_{1}^{\mu_{1}} V_{2}^{\mu_{2}} V_{3}^{\mu_{3}}}{k_{1}^{2} k_{2}^{2} k_{3}^{2}}\left[\hat{a}_{0}+A_{+} \hat{a}_{+}+A_{-} \hat{a}_{-}+H \hat{h}\right] .
$$

Recall from the previous section that $A_{ \pm}, H \propto N_{c}-N_{f}$ in QCD.

The three-gluon effective coupling evolves according to

$$
\tilde{\alpha}(a, b, c)=\frac{\widetilde{\alpha}\left(a_{0}, b_{0}, c_{0}\right)}{1-2\left(\tilde{A}_{0}(a, b, c)-\widetilde{A}_{0}\left(a_{0}, b_{0}, c_{0}\right)\right)}
$$


In four dimensions with regularization scheme $R=D R E D$ or $D R E G$ we have

$$
\begin{aligned}
\widetilde{A}_{0}(a, b, c) & =-\frac{\alpha_{s}}{8 \pi} \beta_{0}\left[L(a, b, c)-\log \mu^{2}-C_{U V}-\eta_{3}\right] \\
\text { where } \beta_{0} & =\frac{11}{3} N_{c}-\frac{2}{3} N_{f}-\frac{1}{6} N_{s} \\
C_{U V} & =\frac{1}{\epsilon}-\gamma_{E}+\log 4 \pi \\
\eta_{3} & =(2+\Omega)+\frac{N_{c}}{3 \beta_{0}} \delta_{R, D R E G} \\
\Omega & =\frac{16}{3 \sqrt{3}} \mathrm{Cl}_{2}(\pi / 3) \approx 3.125
\end{aligned}
$$

The scheme dependence $\delta_{R, D R E G}$ is explained in more detail in Appendix D. Here we have defined

$$
L(a, b, c)=\frac{1}{\mathcal{K}}(\alpha \gamma \log a+\alpha \beta \log b+\beta \gamma \log c-a b c \bar{J}(a, b, c))+\Omega,
$$

and the (massless) triangle integral function $\bar{J}=\bar{J}(a, b, c)=-16 i \pi^{2} J(a, b, c)$ is given in Appendix B in terms of Clausen functions, Eqs.(5.121,5.122). This result (Eqs. $(5.69,5.72)$ ) differs from Lu [83] by only the finite constants which (slightly) affects the numerical extraction from data. The discrepancy can be traced to the inconsistent application of dimensional regularization in [83].

The logarithm-like function $L$ satisfies

$$
L(a, a, a)=\log a
$$

since

$$
\bar{J}(a, a, a)=\frac{4}{a \sqrt{3}} \mathrm{Cl}_{2}(\pi / 3) .
$$

One can use the real part of this function to define an effective scale of the three-gluon vertex:

$$
\begin{aligned}
L(a, b, c) & =\log \left(Q_{e f f}^{2}(a, b, c)\right)+i \operatorname{Im} L(a, b, c) \\
Q_{e f f}^{2}(a, b, c) & =|a|^{\alpha \gamma / \mathcal{K}}|b|^{\alpha \beta / \mathcal{K}}|c|^{\beta \gamma / \mathcal{K}} \exp \left(\Omega-\frac{a b c}{\mathcal{K}} \operatorname{Re} \bar{J}(a, b, c)\right) .
\end{aligned}
$$


This is sensible since the dimensions of $Q_{e f f}^{2}(a, b, c)$ are indeed mass squared.

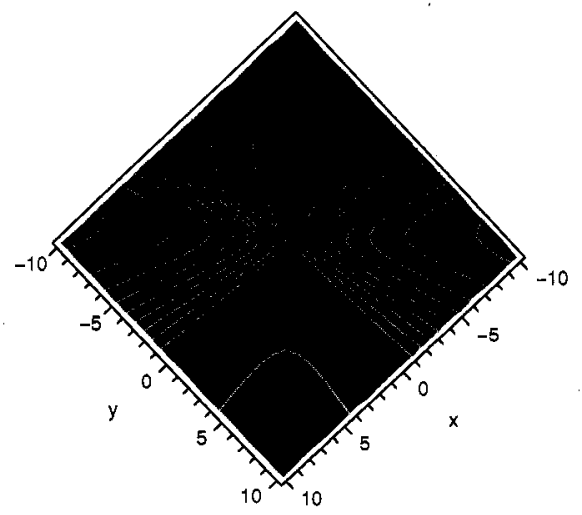

Figure 5.5: A contour plot of $Q_{e f f}^{2}(1, x, y)$. The contours, from red to blue, are at $2,4,6,8,10,12,14,16,18,20$.

The three-gluon effective charge $\widetilde{\alpha}(a, b, c)$ is related to the usual $\overline{M S}$ coupling $\bar{\alpha}\left(q^{2}\right)$ by

$$
\begin{aligned}
\frac{1}{\widetilde{\alpha}(a, b, c)} & =\frac{1}{\bar{\alpha}\left(\mu^{2}\right)}+\frac{\beta_{0}}{4 \pi}\left(\log \frac{Q_{e f f}^{2}(a, b, c)}{\mu^{2}}+i \operatorname{Im} L(a, b, c)-\eta_{3}\right) \\
& =\frac{1}{\bar{\alpha}\left(e^{-\eta_{3}} Q_{e f f}^{2}(a, b, c)\right)}+i \frac{\beta_{0}}{4 \pi} \operatorname{Im} L(a, b, c) .
\end{aligned}
$$

Since $\exp \left(\frac{\eta_{3}}{2}\right) \approx 14$, we see that when using $\overline{M S}$, the scale should be fourteen times lower than the typical virtualities of the gluons, given by $Q_{e f f}(a, b, c)$. Of course, this is true only if the three-gluon vertex diagram dominates the physical process. In general there will be different scales at the various quark-gluon vertices when using the PT scheme (as seen in Eq.(5.70)). In contrast, in $\overline{M S}$ the same scale is used at every vertex. The following approximate values of the three-gluon coupling are derived from Eq.(5.77) (including the effects of quark masses which discussed in the 


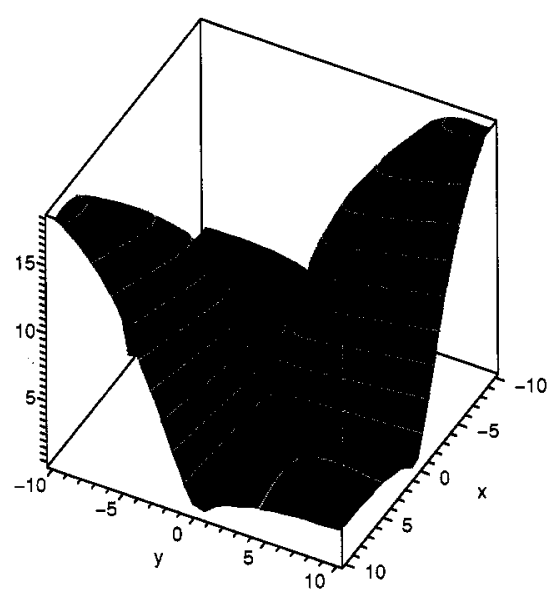

Figure 5.6: A three-dimensional contour plot of $Q_{e f f}^{2}(1, x, y)$.

next section) for various symmetric timelike( $\mathrm{T})$ and spacelike(S) configurations :

$$
\begin{array}{ll}
\text { SSS : } & \widetilde{\alpha}\left(-M_{Z}^{2},-M_{Z}^{2},-M_{Z}^{2}\right) \approx 0.192 \\
\text { SST : } & \widetilde{\alpha}\left(-M_{Z}^{2},-M_{Z}^{2},+M_{Z}^{2}\right) \approx 0.157+0.023 I \\
\text { STT : } & \widetilde{\alpha}\left(-M_{Z}^{2},+M_{Z}^{2},+M_{Z}^{2}\right) \approx 0.156+0.025 I \\
\text { TTT : } & \widetilde{\alpha}\left(+M_{Z}^{2},+M_{Z}^{2},+M_{Z}^{2}\right) \approx 0.170+0.062 I
\end{array}
$$

It is clear that the three-gluon coupling is stronger than naively expected from $\alpha_{\overline{M S}}\left(M_{Z}\right) \approx 0.118$.

The effective scale $Q_{\text {eff }}^{2}(a, b, c)$ satisfies the following relations:

$$
\begin{aligned}
Q_{e f f}^{2}(a, b, c) & =Q_{e f f}^{2}(-a,-b,-c) \\
Q_{e f f}^{2}(\lambda a, \lambda b, \lambda c) & =|\lambda| Q_{e f f}^{2}(a, b, c) \\
Q_{e f f}^{2}(a, a, a) & =|a| \\
Q_{e f f}^{2}(a,-a,-a) & \approx 5.54|a| \\
Q_{e f f}^{2}(a, a, c) & \approx|c| e^{\Omega-2} \quad \text { for }|a| \gg|c| \\
Q_{e f f}^{2}(a,-a, c) & \approx|c| e^{\Omega} \quad \text { for }|a| \gg|c| \\
Q_{e f f}^{2}(a, b, c) & \approx \frac{|b||c|}{|a|} e^{\Omega} \quad \text { for }|a| \gg|b|,|c| .
\end{aligned}
$$


$\mathrm{Lu}[83]$ has previously found the last of these limits in the case where all momenta are spacelike, giving an effective scale $Q_{\min } Q_{\operatorname{med}} / Q_{\max }$. It should be noted that the rate of convergence to the above limits strongly depends on the signatures $(\mathrm{S} \equiv$ spacelike $\leftrightarrow$ $p^{2}<0, \mathrm{~T} \equiv$ timelike $\leftrightarrow p^{2}>0$ ) of the virtualities $a, b, c$. If the signatures are mixed (TTS) or (TSS) then the convergence is very slow, and the effective scale tends to stay larger compared to the cases (SSS) or (TTT).

Some plots demonstrating the novel behavior of $Q_{e f f}^{2}$ are given in Figs. $(5.3,5.4,5.5,5.6)$.

\subsection{Phenomenological Effects of Internal Masses}

So far, all fields propagating in the triangle graphs have been treated as massless. This was useful for simplifying the discussion and elucidating the general structure of the radiative corrections and the $N=4$ sum rules. However, in real world applications one usually does not have all three gluon virtualities in the same desert region $M_{i} \ll$ $a, b, c \ll M_{i+1}$. Thus, mass corrections should be taken into account. We have calculated the effects of massive fermions (MQ), massive scalars (MS), and massive gauge bosons (MG) for all of the form factors; the complete results are given in Appendix E. The corrections for the case of massive fermions were first obtained in Ref.[84] and we are in agreement. Here we will focus on the massive quark $(M Q)$ contribution to the form factor multiplying the tree level tensor structure, which from Appendix $\mathrm{E}$ and section 3 is

$$
A_{0}(M Q)=\frac{4 M^{2}}{3(d-2)} J_{M}+\frac{3 d-8}{6 \mathcal{K}(d-2)}\left[a b c J_{M}+a \beta J_{1 M}+b \gamma J_{2 M}+c \alpha J_{3 M}\right] .
$$

Here $J_{M}, J_{1 M}, J_{2 M}$, and $J_{3 M}$ are the massive analogs of $J, J_{1}, J_{2}$ and $J_{3}$, respectively. The two-point function $J_{1 M}$ and tadpole $T_{M}$ are reviewed in Appendix A, while Appendix $\mathrm{B}$ is devoted to a discussion of the massive triangle integral, $J_{M}$, and its analytic continuations and various limits.

As in the previous section, when considering a physical matrix element we always have the combination $\widetilde{A}_{0}=A_{0}-\frac{1}{2}\left(\Pi_{1}+\Pi_{2}+\Pi_{3}\right)$ multiplying the tree-level tensor structure. This leads us to consider the massive quark contribution $\widetilde{A}_{0}(M Q)=$ 


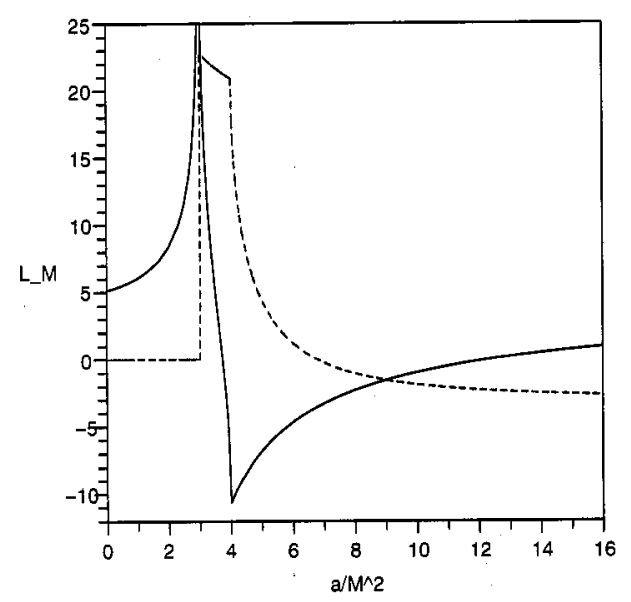

Figure 5.7: $L_{M Q}\left(a / M^{2}, a / M^{2}, a / M^{2}\right)$ vs. $a / M^{2}$ for timelike $a>0$. The solid line is the real part and the dashed line is the imaginary part.

$A_{0}(M Q)-\frac{1}{2}\left(\Pi_{1}(M Q)+\Pi_{2}(M Q)+\Pi_{3}(M Q)\right)$, which upon using Eq. (5.135), inserting the prefactor $i g^{2}$, and expanding around $d=4$ becomes

$$
\begin{aligned}
\widetilde{A}_{0}(M Q) & =-\frac{\alpha_{s}}{4 \pi}\left[\frac{1}{3}\left(C_{U V}-\log \frac{M^{2}}{\mu^{2}}\right)+\frac{1}{3 \mathcal{K}}\left(a b c \overline{J_{M}}-\alpha \gamma \mathcal{L}(a)-\alpha \beta \mathcal{L}(b)-\beta \gamma \mathcal{L}(c)\right)\right. \\
& \left.+\frac{2}{3}+\frac{2 M^{2}}{3}\left(\overline{J_{M}}+\frac{2-\mathcal{L}(a)}{a}+\frac{2-\mathcal{L}(b)}{b}+\frac{2-\mathcal{L}(c)}{c}\right)\right]
\end{aligned}
$$

Here $\overline{J_{M}}=-16 i \pi^{2} J_{M}$ and

$$
\mathcal{L}(a)=v(a) \log \frac{v(a)+1}{v(a)-1} \text { where } v(a)=\sqrt{1-\frac{4\left(M^{2}-i \epsilon\right)}{a}}
$$




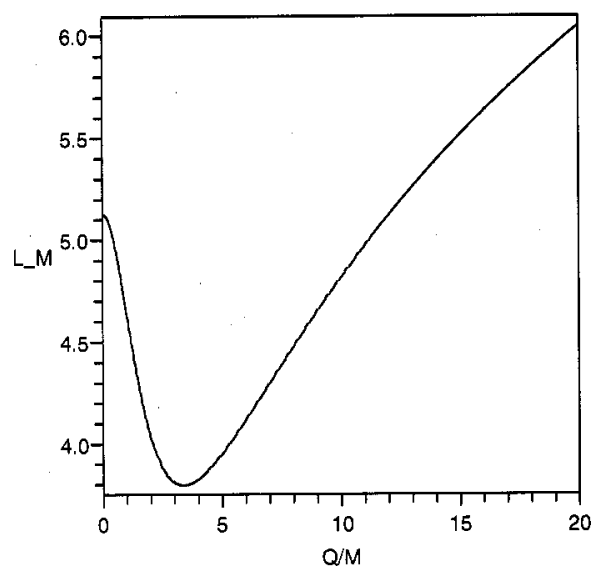

Figure 5.8: $L_{M Q}\left(-Q^{2} / M^{2},-Q^{2} / M^{2},-Q^{2} / M^{2}\right)$ vs. $Q / M$ for spacelike $-Q^{2}<0$.

comes from $J_{1 M}$ and has the analytic continuations given in Eq.(5.106). The threescale logarithm-like function for massive quarks (MQ) is thus given by

$$
\begin{aligned}
L_{M Q}\left(\frac{a}{M^{2}}, \frac{b}{M^{2}}, \frac{c}{M^{2}}\right) & =\frac{1}{\mathcal{K}}\left(\alpha \gamma \mathcal{L}(a)+\alpha \beta \mathcal{L}(b)+\beta \gamma \mathcal{L}(c)-a b c \overline{J_{M}}(a, b, c)\right)+\Omega \\
& +2 M^{2}\left(\frac{\mathcal{L}(a)-2}{a}+\frac{\mathcal{L}(b)-2}{b}+\frac{\mathcal{L}(c)-2}{c}-\overline{J_{M}}\right) \\
\widetilde{A}_{0}(M Q) & =\left(\frac{\alpha_{s}}{12 \pi}\right)\left[L_{M}\left(\frac{a}{M^{2}}, \frac{b}{M^{2}}, \frac{c}{M^{2}}\right)+\log \frac{M^{2}}{\mu^{2}}-C_{U V}-(2+\Omega)\right] .
\end{aligned}
$$

This massive logarithm-like function has the following limits :

$$
\begin{aligned}
& L_{M Q}\left(\frac{a}{M^{2}}, \frac{b}{M^{2}}, \frac{c}{M^{2}}\right) \approx 2+\Omega \quad M^{2} \gg|a|,|b|,|c| \\
& L_{M Q}\left(\frac{a}{M^{2}}, \frac{b}{M^{2}}, \frac{c}{M^{2}}\right) \approx L(a, b, c)-\log M^{2} \quad M^{2} \ll|a|,|b|,|c|,
\end{aligned}
$$

with the number $\Omega \approx 3.125$ defined in Eq.(5.72). The convergence to the massless limit is very slow, indicating that threshold effects must be included for most applications.

In Figs. $(5.7,5.8)$ we have plotted $L_{M Q}$ for the symmetric case $a=b=c$ for timelike and spacelike momenta. For the timelike case, the threshold at $a=4 M^{2}$ 


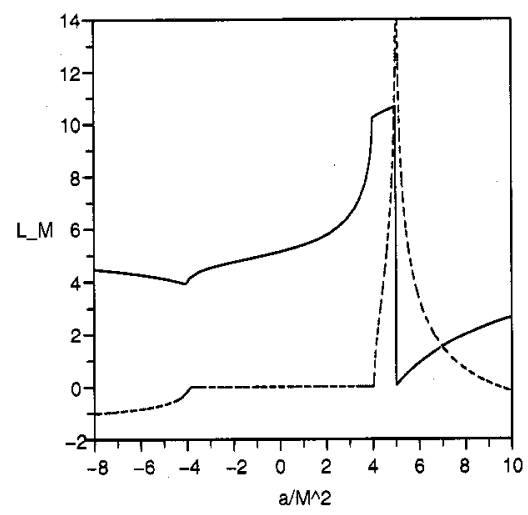

Figure 5.9: $L_{M Q}\left(a / M^{2}, a / M^{2},-a / M^{2}\right)$ vs. $a / M^{2}$. The solid line is the real part and the dashed line is the imaginary part. The real thresholds are at $a= \pm 4 M^{2}$ while the pseudo-threshold is at $a=5 M^{2}$.

and the pseudo-threshold at $a=3 M^{2}$ are evident. In Fig.(5.9) the mixed case (TTS) $L_{M Q}\left(a / M^{2}, a / M^{2},-a / M^{2}\right)$ is plotted, where the thresholds at $a= \pm 4 M^{2}$ and the pseudo-threshold at $a=5 M^{2}$ are evident. For the purely timelike (TTT) case in Fig. (5.7), there is a discontinuity in the imaginary part and the real part diverges at the pseudo-threshold. In contrast, for the mixed signature case (TTS) the imaginary part diverges while the real part is discontinuous. This pseudo-threshold phenomena is explained in more detail in Appendix B.

From the above results, one can define the effective number of active quarks which characterizes the effects of quark mass :

$$
N_{F}\left(\frac{a}{M^{2}}, \frac{b}{M^{2}}, \frac{c}{M^{2}}\right)=-M^{2} \frac{d}{d M^{2}} L_{M Q}\left(\frac{a}{M^{2}}, \frac{b}{M^{2}}, \frac{c}{M^{2}}\right) .
$$

This clearly goes to zero and one in the limits $M^{2} \gg|a|,|b|,|c|$ and $M^{2} \ll|a|,|b|,|c|$, respectively.

To motivate this definition, let's. look at the single-scale pinch-technique effective 


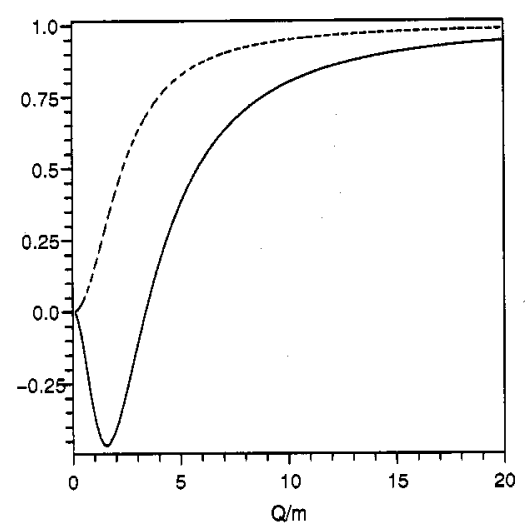

Figure 5.10: The effective number of quark flavors. The lower blue solid curve is $N_{F}\left(Q^{2} / M^{2}\right)$ for the symmetric spacelike $\left(a=b=c=-Q^{2}\right)$ three-gluon vertex, while the upper dashed red curve is the fermion number of flavors $N_{1 / 2}\left(Q^{2} / M^{2}\right)$ for the single scale effective charge.

charge (using the notation of [1]) as a function of spacelike momenta $a=-Q^{2}$

$$
\frac{1}{\widetilde{\alpha}\left(Q^{2}\right)}=\frac{1}{\alpha_{0}}+\frac{1}{4 \pi} \sum_{p} \beta_{p}\left(L_{p}\left(Q^{2} / m_{p}^{2}\right)-\log \frac{\mu^{2}}{m_{p}^{2}}-C_{U V}-\eta_{p}\right)
$$

where $\beta_{p}$ is the contribution of each particle $p$ to the first $\beta$ function coefficient, and to a very good numerical approximation (for spacelike momenta)

$$
L_{p}\left(Q^{2} / m_{p}^{2}\right) \approx \log \left(e^{\eta_{p}}+\frac{Q^{2}}{m_{p}^{2}}\right)
$$

where the constants $\eta_{p}$ are $5 / 3,8 / 3$, and $40 / 21$ for massive fermions, scalars, and gauge bosons, respectively. The exact one-loop formula are given in Eq.(23-26) of Ref.[1], although the analytic continuation in Eq.(26) of that paper should have opposite 
imaginary part. This effective charge satisfies the RGE

$$
\begin{aligned}
\frac{d \widetilde{\alpha}\left(Q^{2}\right)}{d \log Q^{2}} & =-\frac{\widetilde{\alpha}^{2}}{4 \pi} \sum_{p} \beta_{p} \frac{d L_{p}\left(Q^{2} / m_{p}^{2}\right)}{d \log Q^{2}} \equiv-\frac{\widetilde{\alpha}^{2}}{4 \pi} \sum_{p} \beta_{p} N_{p}\left(\frac{Q^{2}}{m_{p}^{2}}\right) \\
N_{p}\left(\frac{Q^{2}}{m_{p}^{2}}\right) & \equiv \frac{d L_{p}\left(Q^{2} / m_{p}^{2}\right)}{d \log Q^{2}}=-\frac{d L_{p}\left(Q^{2} / m_{p}^{2}\right)}{d \log m_{p}^{2}} \approx \frac{1}{1+\frac{m_{p}^{2}}{Q^{2}} e^{\eta_{p}}} .
\end{aligned}
$$

The function $N_{p}$ goes to one when $Q^{2} \gg m_{p}^{2}$ and zero when $Q^{2} \ll m_{p}^{2}$ and unambiguously measures what fraction of particle $p$ is "turned on" at scale $Q^{2}$.

Moving back to the three-scale case, we now have the complication that our effective charge is a solution of a multi-scale RGE

$$
\frac{d \widetilde{\alpha}(a, b, c)}{d \log a}=-\frac{\widetilde{\alpha}^{2}}{4 \pi} \sum_{p} \beta_{p} \frac{d}{d \log a} L_{M Q}\left(\frac{a}{M^{2}}, \frac{b}{M^{2}}, \frac{c}{M^{2}}\right),
$$

and two other permutations with $a \rightarrow b$ or $a \rightarrow c$. This leads to three different $N_{f}$ 's :

$$
N_{f}\left(\frac{a}{M^{2}} \mid \frac{b}{M^{2}}, \frac{c}{M^{2}}\right)=\frac{d}{d \log a} L_{M Q}\left(\frac{a}{M^{2}}, \frac{b}{M^{2}}, \frac{c}{M^{2}}\right),
$$

and two cyclic permutations, each of which goes to $1 / 3$ in the symmetric desert $a=b=c \gg M^{2}$. This suggests adding all three together to define a symmetric

$$
N_{F}\left(\frac{a}{M^{2}}, \frac{b}{M^{2}}, \frac{c}{M^{2}}\right)=\left(\frac{d}{d \log a}+\frac{d}{d \log b}+\frac{d}{d \log c}\right) L_{M Q}\left(\frac{a}{M^{2}}, \frac{b}{M^{2}}, \frac{c}{M^{2}}\right),
$$

which is in fact the same as given in Eq.(5.84).

The results for $N_{F}$ can be obtained with the help of the results in Appendix B. Instead of presenting these lengthy results, let us focus on the symmetric case $a=b=c$, where we find for spacelike $a<0$

$$
\begin{aligned}
N_{F}\left(\frac{a}{M^{2}}\right) & \equiv \frac{d}{d \log a} L_{M Q}\left(\frac{a}{M^{2}}, \frac{a}{M^{2}}, \frac{a}{M^{2}}\right)=-\frac{d}{d \log M^{2}} L_{M Q}\left(\frac{a}{M^{2}}, \frac{a}{M^{2}}, \frac{a}{M^{2}}\right) \\
& =1+18 \frac{M^{2}}{a}+2 M^{2} \overline{J_{M}}(a, a, a)+\frac{54 M^{2} \mathcal{L}(a)\left(a-2 M^{2}\right)}{\left(a-3 M^{2}\right)\left(a-4 M^{2}\right)}
\end{aligned}
$$


In this example, spacelike $a$ is chosen to avoid the pseudo-threshold $a=3 M^{2}$ and the threshold $a=4 M^{2}$. Fig. (5.10) shows a plot of this along with single-scale quark number of flavors function $N_{s=1 / 2}$ from Eq.(5.87).

The negative value of $N_{F}$ at $0 \lesssim Q \lesssim 4 M$ is not entirely novel, as a similar behavior was also found in the context of two-loop quark mass corrections to $\mathrm{V}$ scheme effective charge [85]. It is essentially due to the anti-screening of color charge, this case in the triangle interaction, and does not arise in the one-loop single scale effective charge, as seen in Fig. (5.9).

Using the results of Appendix E, the above analysis can be easily extended to the case of massive scalars (MS) or massive gauge bosons (MG), which have the following logarithm-like functions

$$
\begin{aligned}
L_{M S}\left(\frac{a}{M^{2}}, \frac{b}{M^{2}}, \frac{c}{M^{2}}\right) & =\frac{1}{\mathcal{K}}\left(\alpha \gamma \mathcal{L}(a)+\alpha \beta \mathcal{L}(b)+\beta \gamma \mathcal{L}(c)-a b c \overline{J_{M}}(a, b, c)\right)+\Omega \\
& -4 M^{2}\left(\frac{\mathcal{L}(a)-2}{a}+\frac{\mathcal{L}(b)-2}{b}+\frac{\mathcal{L}(c)-2}{c}-\overline{J_{M}}\right) \\
L_{M G}\left(\frac{a}{M^{2}}, \frac{b}{M^{2}}, \frac{c}{M^{2}}\right) & =\frac{1}{\mathcal{K}}\left(\alpha \gamma \mathcal{L}(a)+\alpha \beta \mathcal{L}(b)+\beta \gamma \mathcal{L}(c)-a b c \overline{J_{M}}(a, b, c)\right)+\Omega \\
& +\frac{4}{7} M^{2}\left(\frac{\mathcal{L}(a)-2}{a}+\frac{\mathcal{L}(b)-2}{b}+\frac{\mathcal{L}(c)-2}{c}-\overline{J_{M}}\right) .
\end{aligned}
$$

The qualitative behavior is the same as the quark case.

Finally, we should consider the limitations of the effective scale $Q_{e f f}^{2}(a, b, c)$ introduced in the last section and effective number of flavors $N_{F}\left(a / M^{2}, b / M^{2}, c / M^{2}\right)$ discussed in this section. Given the complicated structure of the full mass dependent form factors, such tools for characterizing and understanding the behavior of the vertex are helpful. However, in a real calculation such methods may be of limited use and the full mass dependent results should be used. For example, the effective scale $Q_{e f f}^{2}$ has been defined only in the massless case so far because the definition becomes complicated and somewhat arbitrary in the massive case. In particular, consider the 
possible definition (for QCD):

$$
\begin{aligned}
& \operatorname{Re}\left[\frac{11}{3} C_{A} L(a, b, c)-\frac{2}{3} \sum_{q}\left(L_{M Q}\left(\frac{a}{M_{q}^{2}}, \frac{b}{M_{q}^{2}}, \frac{c}{M_{q}^{2}}\right)+\log M_{q}^{2}\right)\right] \\
& \equiv\left(\frac{11 C_{A}}{3}-\frac{2}{3} \sum_{q} \widetilde{N_{q}}\left(\frac{a}{M_{q}^{2}}, \frac{b}{M_{q}^{2}}, \frac{c}{M_{q}^{2}}\right)\right) \log \widetilde{Q}_{e f f}^{2}(a, b, c),
\end{aligned}
$$

where $\widetilde{N_{q}}$ is some suitably defined number of flavors, possibly a step function such as $\delta\left(a+b+c-3 M_{q}^{2}\right)$, possibly the $N_{F}$ defined in Eq.(5.84), or some other definition. It should be clear that any choice of $\widetilde{N}_{q}$ determines $\widetilde{Q}_{\text {eff }}^{2}$, and vice versa, and there seems to be no compelling choice for these quantities. Furthermore, in the approach advocated here, the couplings at each vertex depend on physical momentum scales which will typically be integrated over in the phase space. Thus, matching onto a conventional $\overline{M S}$ type approach can only be done at the end of the calculation, so that trying to define a $Q_{e f f}^{2}$ at an intermediate stage is not very useful.

Thus, in real-world applications, one should generally use the full results for the mass dependent form factors. This constitutes a multi-scale analytic renormalization scheme that contains information which cannot be obtained in the simple singlescale leading-log renormalization methods. In other words, every three-gluon vertex (at tree-level) can be dressed, or "RG improved", with this gauge-invariant effective coupling and the associated form factors, which are process independent and contain more information than the $\overline{M S}$ procedure.

\subsection{Conclusions and Future Directions}

The results of this chapter represent only a fraction of what is needed for a reorganization of perturbation theory into fully gauge-invariant pieces with physical content, each of which can be renormalized independently, leading naturally to a physical multi-scale analytic renormalization scheme. This is possible due to the remarkable properties of the pinch technique (PT)/ Background Field Method quantum Feynman gauge (BFMFG) Green's functions. There is still much progress that can 
be made in calculating these Green's functions in perturbation theory.

The present chapter gives a complete and general characterization of the off-shell three-gluon vertex at one-loop. A similar study of the gauge-invariant triple gauge boson vertices of the Standard Model [66] would be very useful. It may also be possible to quantitatively look at the unification of triple gauge boson vertices and couplings, in analogy with the work on the unification of single-scale PT couplings[1]. Some progress has been made on the conventional gauge-dependent three-gluon vertex at two loops [86] [87], which gives hope for eventually treating the gauge-invariant threegluon vertex at two-loops.

The gauge-invariant PT/BFMFG quark self-energy turns out to be equal to the conventional self-energy in the Feynman gauge [75], and so is known through twoloops [88]. Due to the Ward identity [36] satisfied by the PT/BFMFG quark-gluon vertex, this also yields the longitudinal form factors of $\Gamma_{\mu}^{(q q g)}$ through two loops.

In $\mathrm{QCD}$, another logical step is the four-gluon vertex at one-loop. In the general off-shell case, there are hundreds of independent tensors structures and form factors.

Beyond perturbation theory, the study of Dyson-Schwinger Equations [61] and renormalons [63] in the PT/BFMFG approach may yield new insight.

To summarize, in this chapter we have analyzed the behavior of the gaugeinvariant three-gluon vertex at one-loop. Starting from the symmetry principles governing the vertex, a convenient tensor basis decomposition was given in Eqs. $(5.7,5.12)$. As seen in Eq.(5.65) and the subsequent discussion, this basis is the most convenient for phenomenological studies, since it is built out of "transverse" $(-)$ and "longitudinal" $(+)$ momenta, the latter of which vanish when dotted into external on-shell vertices, thus leading to relatively simple matrix elements. In the case considered in section 5.4, only four form factors remain, rather than the thirteen which would be present in a generic basis. Nonetheless, the choice of basis is only a matter of convenience, and the real physics lies in the thirteen non-vanishing form factors given explicitly in section 5.3.

The supersymmetric relations between the scalar, quark, and gluon contributions leads to a simple presentation of the results for a generic (unbroken) gauge theory. Only the gluon contributions to the form factors are given explicitly in section 5.3, 
while the quark and scalar contributions are inferred from the homogeneous relation $F_{G}+4 F_{Q}+(10-d) F_{S}=0$ and the results for the relatively simple sums $\Sigma_{Q G}(F) \equiv$ $\frac{(d-2)}{2} F_{Q}+F_{G}$ which are given in section 5.3 for each form factor $F$. The extension to the case of internal masses is outlined in Appendix $\mathrm{E}$ and leads to the modified sum rule $F_{M G}+4 F_{M Q}+(9-d) F_{M S}=0$.

The phenomenology is largely determined by the form factor of the tree-level tensor structure, which in section 5.4 is used to define a three-scale effective charge $\widetilde{\alpha}(a, b, c)$. In addition, the characteristic scale $Q_{e f f}^{2}(a, b, c)$ governing the behavior of the vertex and the effective charge was analyzed, thus providing a natural extension of BLM scale setting [12] to the three-gluon vertex. Physical momentum scales always set the scale of the coupling. The phenomenological effects of quark masses are discussed in section 5.5 and are found to be important for generic physical applications, since decoupling is slow and a complicated threshold and pseudo-threshold behavior is observed. An important next step is to fully apply these techniques to a physical process. In the future we will present such an analysis for the hadronic production of heavy quarks, where the importance of the form factors other than the tree-level one $\left(A_{0}\right)$ will be addressed. The interpretation of the pseudo-threshold phenomena also deserves further study.

Acknowledgements I would like to thank Lance Dixon for useful discussions regarding the second order formalism of the BFM and the supersymmetric decomposition of one-loop amplitudes. 


\subsection{Appendix A : Reduction to Scalar Integrals}

First, we will describe the evaluation of the massless integrals, and then briefly mention the modifications due to internal masses. As before, we will use the shorthand notation

$$
a=p_{1}^{2} \quad b=p_{2}^{2} \quad c=p_{3}^{2} \quad \alpha=p_{1} \cdot p_{2} \quad \beta=p_{2} \cdot p_{3} \quad \gamma=p_{3} \cdot p_{1}
$$

In order to evaluate the integrals in an efficient manner, it is very convenient to choose a manifestly symmetric routing of the loop momenta, as shown in Fig.5.1, where clearly

$$
l_{1}=p_{2}+l_{3} \quad l_{2}=p_{3}+l_{1} \quad l_{3}=p_{1}+l_{2} .
$$

Of course there is only one integration momenta $l$, which can be chosen to be $l_{1}, l_{2}$, or $l_{3}$, thus breaking the cyclic symmetry. However, using the symmetric labeling greatly simplifies the analysis.

First we decompose the full vertex $\Gamma$ into longitudinal $(L)$ and transverse $(T)$ parts, $\Gamma=\Gamma_{L}+\Gamma_{T}$, as in Eq.(5.14). The tensor integrals in Eq. (5.18) are then converted into scalar integrals by applying projection operators. In doing so, the longitudinal $(L)$ and transverse $(T)$ parts essentially decouple, and the ten independent $L$ form factors are easily found either directly, or by solving the Ward ID, resulting in Eq.(6.22). The remaining four $T$ parts are found by applying the following four projection operators to Eq.(5.18) : 200,030,001, and 231, where as in Table 5.1 we have defined $030 \equiv$ $p_{3 \mu_{2}} g_{\mu_{1} \mu_{3}}$, etc. Thus, for the gluon contribution $G$ we have four scalar integrals : $G(200) \equiv p_{2 \mu_{1}} g_{\mu_{2} \mu_{3}} G_{\mu_{1} \mu_{2} \mu_{3}}, G(030), G(001)$, and $G(231)$. Similarly, there are four integrals for the quarks and scalars as well. In the numerator of each of these integrals there will be various dot products of momenta, which can always be reduced to momentum squares using Eq.(5.96). For example, $p_{1} \cdot l_{2}=\left(l_{3}^{2}-l_{2}^{2}-p_{1}^{2}\right) / 2$ and $l_{1} \cdot l_{3}=$ $\left(l_{1}^{2}+l_{3}^{2}-p_{2}^{2}\right) / 2$. Thus we are left with integrals of the form

$$
I_{i j k}=\int \frac{\left(l_{1}^{2}\right)^{i}\left(l_{2}^{2}\right)^{j}\left(l_{3}^{2}\right)^{k}}{l_{1}^{2} l_{2}^{2} l_{3}^{2}}
$$


where $\int \equiv \int \frac{d^{d} l}{(2 \pi)^{d}}$ and $i, j, k \in\{0,1,2\}$. Using the standard rules of dimensional regularization, it is easy to see that any integral with any two of $i, j, k$ nonzero must vanish. Furthermore, it is straightforward to show that

$$
I_{200}=-\beta I_{100} \quad I_{020}=-\gamma I_{010} \quad I_{002}=-\alpha I_{001} .
$$

Thus we are left with only two types of integrals: (1) the trivial two point integrals $J_{1}, J_{2}$, and $J_{3}$, where

$$
J_{1}=I_{100}=\int \frac{1}{l_{2}^{2} l_{3}^{2}}=\int \frac{1}{l^{2}\left(l+p_{1}\right)^{2}}
$$

and (2) the master triangle integral

$$
J \equiv J\left(p_{1}^{2}, p_{2}^{2}, p_{3}^{2}\right)=\int \frac{1}{l_{1}^{2} l_{2}^{2} l_{3}^{2}}
$$

For the gluon contribution, for example, one then has a system of four equations with four unknowns, the transverse form factors. Denoting the gluon contribution to the longitudinal projections by $L_{G}(200)=200 \cdot \Gamma_{L}(G)$, etc. we solve for the transverse form factors

$$
\begin{aligned}
& \left(\begin{array}{c}
\bar{F}_{12}(G) \\
\bar{F}_{23}(G) \\
\bar{F}_{31}(G) \\
\bar{H}(G)
\end{array}\right)=M_{T}^{-1}\left(\begin{array}{c}
G(200)-L_{G}(200) \\
G(030)-L_{G}(030) \\
G(001)-L_{G}(001) \\
G(231)-L_{G}(231)
\end{array}\right) \\
& \text { where } M_{T}=-\mathcal{K}\left(\begin{array}{cccc}
\beta & (d-1) \beta & \beta & 2-d \\
\gamma & \gamma & (d-1) \gamma & 2-d \\
(d-1) \alpha & \alpha & \alpha & 2-d \\
\mathcal{K} & \mathcal{K} & \mathcal{K} & 0
\end{array}\right),
\end{aligned}
$$

and similarly for the quark and scalar contributions.

The above procedure can also be followed for the massive case, with only a few modifications. First, the tadpole $M^{2} T_{M}=\int \frac{1}{l_{i}^{2}-M^{2}}$ does not vanish. Thus, instead of 
$\int \frac{l_{1}^{2}}{l_{2}^{2} l_{3}^{2}}=-\beta J_{1}$ we now have

$$
\int \frac{l_{1}^{2}-M^{2}}{\left(l_{2}^{2}-M^{2}\right)\left(l_{3}^{2}-M^{2}\right)}=-\beta J_{1 M}+M^{2} T_{M}
$$

where $J_{1 M}=\int \frac{1}{\left(l_{2}^{2}-M^{2}\right)\left(l_{3}^{2}-M^{2}\right)}=\int \frac{1}{\left(l^{2}-M^{2}\right)\left(\left(l+p_{1}\right)^{2}-M^{2}\right)}$. We also need the following result and permutations :

$$
\int \frac{l_{2}^{2}-M^{2}}{l_{3}^{2}-M^{2}}=\int \frac{l_{3}^{2}-M^{2}}{l_{2}^{2}-M^{2}}=a M^{2} T_{M}
$$

Finally, we have the master triangle integral with nonzero masses

$$
J_{M} \equiv J_{M}\left(p_{1}^{2}, p_{2}^{2}, p_{3}^{2}\right)=\int \frac{1}{\left(l_{1}^{2}-M^{2}\right)\left(l_{2}^{2}-M^{2}\right)\left(l_{3}^{2}-M^{2}\right)}
$$

To summarize, in the massive case we need $J_{M}, J_{1 M}, J_{2 M}, J_{3 M}$, and $T_{M}$. In the massless case we need $J, J_{1}, J_{2}$, and $J_{3}$. For each of these we pull out the factor $\frac{i}{16 \pi^{2}}$ and define $J_{M}=\frac{i}{16 \pi^{2}} \overline{J_{M}}$, etc.

Some formula for these integrals in $d$ dimensions can be found in [81]. Here we will give only the expansions in four dimensions and define $C_{U V}=\frac{1}{\epsilon}-\gamma_{E}+\log 4 \pi$ where $d=4-2 \epsilon$.

The tadpole integral is

$$
\overline{T_{M}}=C_{U V}+1-\log \frac{M^{2}}{\mu^{2}}
$$

The two point integral is

$$
\begin{aligned}
& \overline{J_{1 M}}=C_{U V}+2-\log \frac{M^{2}}{\mu^{2}}-\mathcal{L}(a) \\
& \mathcal{L}(a)=\left\{\begin{array}{l}
2 v \tanh ^{-1}\left(v^{-1}\right)=v \log \frac{v+1}{v-1} \\
2 \bar{v} \tan ^{-1}\left(\bar{v}^{-1}\right) \\
2 v \tanh ^{-1}(v)-i \pi v=v \log \frac{1+v}{1-v}-i \pi v
\end{array}\right\} \text { for }\left\{\begin{array}{l}
a<0 \\
0<a<4 M^{2} \\
a>4 M^{2}
\end{array}\right\}
\end{aligned}
$$


and the generalized velocities are

$$
v=\sqrt{1-\frac{4 M^{2}}{a}} \quad \bar{v}=\sqrt{\frac{4 M^{2}}{a}-1} .
$$

In the massless limit this becomes

$$
\overline{J_{1}}=C_{U V}+2-\log \frac{|a|}{\mu^{2}}+i \pi \theta(a) .
$$

\subsection{Appendix B : Results for the Triangle Integral}

The massive triangle integral

$$
J_{M} \equiv J_{M}\left(p_{1}^{2}, p_{2}^{2}, p_{3}^{2}\right)=\int \frac{d^{4} l}{(2 \pi)^{4}} \frac{1}{\left(l_{1}^{2}-M^{2}+i \epsilon\right)\left(l_{2}^{2}-M^{2}+i \epsilon\right)\left(l_{3}^{2}-M^{2}+i \epsilon\right)}
$$

is finite in four dimensions. We will give the results for $\overline{J_{M}}=-i 16 \pi^{2} J_{M}$. This integral has been discussed previously in the literature [89][90][91][84]. In particular, 'tHooft and Veltman [89] derived a formula which is valid for all values of the kinematic variables $a, b, c$ and mass $M$, although careful analytic continuation is required. We will first write the results of [89] in our notation and then discuss the analytic continuations. The various functions involved and some reference formula are summarized below in Appendix C.

Defining $\rho=\sqrt{-\mathcal{K}}$, where as before

$$
\mathcal{K}=\alpha \beta+\beta \gamma+\gamma \alpha=-\frac{1}{4}\left(a^{2}+b^{2}+c^{2}-2(a b+b c+c a)\right),
$$

we have

$$
\overline{J_{M}}=-\frac{1}{2 \rho}\left(I_{3}(a \mid b, c)+I_{3}(b \mid c, a)+I_{3}(c \mid a, b)\right) .
$$

The results for $I_{3}(a \mid b, c)$ can be expressed in terms of the velocity $v=\sqrt{1-\frac{4\left(M^{2}-i \epsilon\right)}{a}}$ 
and the variable $x=\beta / \rho$ :

$$
\begin{aligned}
I_{3}(a \mid b, c) & =\mathrm{Li}_{2}\left(z_{1}\right)-\mathrm{Li}_{2}\left(\overline{z_{1}}\right)+\mathrm{Li}_{2}\left(z_{2}\right)-\mathrm{Li}_{2}\left(\overline{z_{2}}\right)-\eta(x-v, x+v) \log \frac{\overline{z_{2}}}{z_{1}} \\
& +\eta\left(-1-v, \frac{1}{x-v}\right) \log z_{1}-\eta\left(1-v, \frac{1}{x-v}\right) \log \overline{z_{2}} \\
& +\eta\left(-1+v, \frac{1}{x+v}\right) \log z_{2}-\eta\left(1+v, \frac{1}{x+v}\right) \log \overline{z_{1}}
\end{aligned}
$$

where we have defined

$$
z_{1}=\frac{x+1}{x-v} \quad z_{2}=\frac{x+1}{x+v} \quad \overline{z_{1}}=\frac{x-1}{x+v} \quad \overline{z_{2}}=\frac{x-1}{x-v}
$$

and the function $\eta(x, y)$ compensates for the branch cut in the logarithms:

$$
\begin{aligned}
\log x y & =\log x+\log y+\eta(x, y) \\
\eta(x, y) & =2 i \pi(\theta(-\operatorname{Im} x) \theta(-\operatorname{Im} y) \theta(\operatorname{Im} x \operatorname{Im} y)-\theta(\operatorname{Im} x) \theta(\operatorname{Im} y) \theta(-\operatorname{Im} x \operatorname{Im} y))
\end{aligned}
$$

The other two integrals $I_{3}(b \mid c, a)$ and $I_{3}(c \mid a, b)$ are easily obtained by permutation of the above results, so that $x=\gamma / \rho, v=\sqrt{1-\frac{4 M^{2}}{b}}$ and $x=\alpha / \rho, v=\sqrt{1-\frac{4 M^{2}}{c}}$, respectively. Although these results entirely characterize the massive triangle function, it is a rather tedious exercise to analytically continue the results to the six different physical kinematical regions. To our knowledge, such complete analytic continuations have not appeared in the literature thus far.

$\overline{J_{M}}$ takes different forms for $\mathcal{K}>0$ and $\mathcal{K}<0$ since then the variable $x$ is imaginary and real, respectively. The case $\mathcal{K}>0$ can occur only if all momenta are spacelike $(a, b, c<0)$ or timelike $(a, b, c>0)$. The case $\mathcal{K}<0$ can occur for momenta of any signature. Thus, if all momenta are spacelike or all timelike, the ratios of momenta will determine if $\mathcal{K}>0$ or $\mathcal{K}<0$. For each of these two cases, we must also distinguish when $a$ is spacelike, timelike below threshold, and timelike above threshold. For timelike above threshold and spacelike, the generalized velocity $v=\sqrt{1-\frac{4 M^{2}}{a}}$ is real, except for the $i \epsilon$ term which is used in the analytic continuation and hence not included below. Below threshold $v=i \bar{v}=i \sqrt{\frac{4 M^{2}}{a}-1}$. 


\section{Case $\mathcal{K}>0$}

For $\mathcal{K}>0$ we have

$$
\begin{aligned}
& \rho=\sqrt{-\mathcal{K}}=i \sqrt{\mathcal{K}} \equiv i \bar{\rho} \\
& x=\beta / \rho=-i \beta / \bar{\rho} \equiv-i w
\end{aligned}
$$

- $\mathcal{K}>0$ and $v$ real $\Longleftrightarrow\left(a<0\right.$ or $\left.a>4 M^{2}\right)$

$$
\begin{aligned}
I_{3}(a \mid b, c) & =i\left(2 \mathrm{Cl}_{2}\left(2 \phi_{1}\right)-\mathrm{Cl}_{2}\left(2 \phi_{1}-2 \phi_{2}\right)-\mathrm{Cl}_{2}\left(2 \phi_{1}+2 \phi_{2}\right)\right. \\
& \left.+2 i \pi\left(\phi_{1}-\phi_{2}\right) \theta\left(a-4 M^{2}\right)\right) \\
\phi_{1} & =\tan ^{-1}(w) \quad \phi_{2}=\tan ^{-1}(w / v)
\end{aligned}
$$

- $\mathcal{K}>0$ and $v=i \bar{v} \Longleftrightarrow\left(0<a<4 M^{2}\right)$

$$
\begin{aligned}
I_{3}(a \mid b, c) & =i\left(2 \mathrm{Cl}_{2}\left(2 \bar{\phi}_{1}\right)-\mathrm{Cl}_{2}\left(2 \bar{\phi}_{1}-2 \bar{\phi}_{2}\right)-\mathrm{Cl}_{2}\left(2 \bar{\phi}_{1}+2 \bar{\phi}_{2}\right)\right. \\
& \left.+2 \bar{\phi}_{2} \log \left|\frac{w-\bar{v}}{w+\bar{v}}\right|-2 i \pi \bar{\phi}_{1} \theta(|w|-\bar{v})\right) \\
\bar{\phi}_{1} & =\tan ^{-1}(1 / w) \quad \bar{\phi}_{2}=\tan ^{-1}(1 / \bar{v})
\end{aligned}
$$

Note that the prefactor of $i$ in the above equations cancels against the $i$ from $\rho=i \bar{\rho}$ in the prefactor of Eq.(5.111), so that the terms involving the Clausen function $\mathrm{Cl}_{2}(x)$ (discussed in Appendix C) contribute to the real part of $J_{M}$. 


\section{Case $\mathcal{K}<0$}

Here $x$ is real.

- $\mathcal{K}<0$ and $v$ real $\Longleftrightarrow\left(a<0\right.$ or $\left.a>4 M^{2}\right)$

$$
\begin{aligned}
I_{3}(a \mid b, c) & =\operatorname{Re}\left(\operatorname{Li}_{2}\left(z_{1}\right)-\mathrm{Li}_{2}\left(\overline{z_{1}}\right)+\operatorname{Li}_{2}\left(z_{2}\right)-\operatorname{Li}_{2}\left(\overline{z_{2}}\right)\right) \\
& +2 i \pi\left(\varphi_{1} \sigma(a) \theta(|x|-v)+\varphi_{2} \theta\left(a-4 M^{2}\right)\right) \\
\varphi_{1} & =\frac{1}{2} \log \left|\frac{x+1}{x-1}\right| \quad \varphi_{2}=\frac{1}{2} \log \left|\frac{(x+v)(x-1)}{(x-v)(x+1)}\right|
\end{aligned}
$$

- $\mathcal{K}<0$ and $v=i \bar{v} \Longleftrightarrow\left(0<a<4 M^{2}\right)$

$$
\begin{aligned}
I_{3}(a \mid b, c) & =2 \operatorname{Re}\left(\operatorname{Li}_{2}\left(z_{1}\right)-\mathrm{Li}_{2}\left(\overline{z_{1}}\right)\right) \\
z_{1} & =\frac{x+1}{x-i \bar{v}} \quad \overline{z_{1}}=\frac{x-1}{x+i \bar{v}}
\end{aligned}
$$

Several features of these results deserve comment.

First, in the $\mathcal{K}>0, v=i \bar{v}$ case, there are anomalous thresholds which give rise to a nonzero imaginary part and a diverging real part. As seen in Eq. (5.117), these anomalous thresholds occur in $I_{3}(a \mid b, c)$ (and similarly for $I_{3}(b \mid c, a)$ and $I_{3}(c \mid a, b)$ by permutation) when

$$
|w|=\bar{v} \Longleftrightarrow a b c=4 M^{2} \mathcal{K}
$$

There will be a nonzero imaginary part for $|w|>\bar{v} \Longleftrightarrow a b c>4 M^{2} \mathcal{K}$. Note that since here $4 M^{2}>a>0$ and $\mathcal{K}>0$, we must have $b, c>0$. Let us now look at some special cases:

- $\mathbf{a}=\mathbf{b}=\mathbf{c}$ Here the condition for an anomalous threshold reduces to $a>3 M^{2}$, which was found in [84].

- $\mathrm{b}=\mathrm{c} \quad$ This leads to $\left(a / M^{2}\right)=\left(b / M^{2}\right)\left(4-\left(b / M^{2}\right)\right)$, which is possible only if $b<4 M^{2}$.

There are also anomalous thresholds for the case of $\mathcal{K}<0$ and $a>4 M^{2}$. For example, for the mixed signature symmetric case $a=b=-c>0$, there is a discontinuity in 
the real part of $J_{M}(a, a,-a)$ and a divergence in the imaginary part at $a=5 M^{2}$, as seen in Fig.(5.9). Anomalous thresholds were analyzed long ago [92][93].

In the case $\mathcal{K}>0, v$ real, there is an imaginary part above threshold, $a>4 M^{2}$, which vanishes in the massless limit $\frac{a}{M^{2}} \rightarrow \infty$.

In [91], the authors find an interesting geometrical interpretation and derivation of the triangle integral (and higher $n$-point integrals).

In the symmetric limit $a=b=c$, the above results reduce to those given in Eqs. (55-62) of [84].

In the massless limit, we obtain

- $\mathcal{K}>0$

$$
\begin{aligned}
\bar{J}(a, b, c) & =-\frac{1}{\rho}\left(\mathrm{Cl}_{2}\left(2 \phi_{\alpha}\right)+\mathrm{Cl}_{2}\left(2 \phi_{\beta}\right)+\mathrm{Cl}_{2}\left(2 \phi_{\gamma}\right)\right) \\
\phi_{\alpha} & =\arctan \left(\frac{\rho}{\alpha}\right), \text { etc. }
\end{aligned}
$$

- $\mathcal{K}<0$

$$
\begin{aligned}
\bar{J}(a, b, c) & =-\frac{1}{\rho}\left(\widetilde{\operatorname{Clh}_{2}}\left(2 \phi_{\alpha}\right)+\widetilde{\operatorname{Clh}_{2}}\left(2 \phi_{\beta}\right)+\widetilde{\operatorname{Clh}_{2}}\left(2 \phi_{\gamma}\right)\right. \\
& \left.+i \pi \phi_{\alpha} \theta(a)+i \pi \phi_{\beta} \theta(b)+i \pi \phi_{\gamma} \theta(c)\right) \\
\phi_{\alpha} & =\frac{1}{2} \log \left|\frac{\alpha+\rho}{\alpha-\rho}\right|, \text { etc. }
\end{aligned}
$$

where

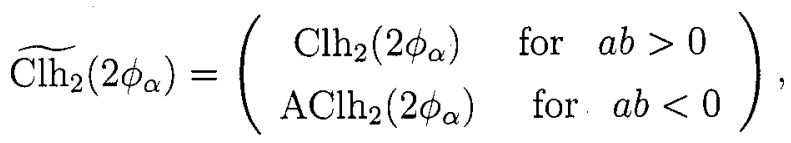

and similarly for $\widetilde{\mathrm{Clh}_{2}}\left(2 \phi_{\beta}\right)$ when $(b c>0, b c<0)$ and $\widetilde{\mathrm{Clh}_{2}}\left(2 \phi_{\gamma}\right)$ when $(c a>$ $0, c a<0)$.

The results for the massless case are well known [89][80][81][83], although the notation is non-standard. Here we have adopted the notation of [83] by using the hyperbolic 
Clausen function $\mathrm{Clh}_{2}(x)$, and alternating hyperbolic Clausen function $\mathrm{AClh}_{2}(x)$, which are discussed below.

\subsection{Appendix C : Special Functions}

Here we collect some useful results, mainly taken from [94]. The dilogarithm function is defined for complex $z$ by

$$
\mathrm{Li}_{2}(z)=-\int_{0}^{z} d x \log \frac{(1-x)}{x} .
$$

In order to find the real and imaginary parts of this function, one should first ensure that the modulus is less than unity by judiciously using

$$
\mathrm{Li}_{2}(z)=-\mathrm{Li}_{2}(1 / z)-\frac{\pi^{2}}{6}-\frac{1}{2} \log ^{2}(-z) .
$$

The notation $\mathrm{Li}_{2}(r, \theta)$, with two arguments, is used for the real part of $\mathrm{Li}_{2}\left(r e^{i \theta}\right)$. For modulus less than unity, $r<1$, we have the integral representation

$$
\mathrm{Li}_{2}(r, \theta)=-\frac{1}{2} \int_{0}^{r} \frac{\log \left(1-2 x \cos \theta+x^{2}\right)}{x}
$$

The imaginary part for $r<1$ is

$$
\begin{aligned}
\operatorname{Im}\left(\mathrm{Li}_{2}\left(r e^{i \theta}\right)\right) & =T \log r+\frac{1}{2}\left(\mathrm{Cl}_{2}(2 \theta)+\mathrm{Cl}_{2}(2 T)-\mathrm{Cl}_{2}(2 \theta+2 T)\right) \\
T & =\tan ^{-1}\left(\frac{r \sin \theta}{1-r \cos \theta}\right) .
\end{aligned}
$$

In particular,

$$
\operatorname{Im}\left(\operatorname{Li}_{2}\left(e^{i \theta}\right)\right)=\mathrm{Cl}_{2}(\theta) \quad \text { and } \quad \mathrm{Cl}_{2}(\theta)=\frac{1}{2 i}\left(\operatorname{Li}_{2}\left(e^{i \theta}\right)-\operatorname{Li}_{2}\left(e^{-i \theta}\right)\right) .
$$


The Clausen function frequently appears in the triangle integral and has the following representations :

$$
\mathrm{Cl}_{2}(x)=-\int_{0}^{x} d y \log \left|2 \sin \frac{y}{2}\right|=\sum_{n=1}^{\infty} \frac{\sin n x}{n^{2}}
$$

Furthermore, $\mathrm{Cl}_{2}(x)$ is odd, $\mathrm{Cl}_{2}(-x)=-\mathrm{Cl}_{2}(x)$, satisfies periodicity, $\mathrm{Cl}_{2}(x+2 n \pi)=$ $\mathrm{Cl}_{2}(x)$, and a duplication formula $\mathrm{Cl}_{2}(2 x)=2 \mathrm{Cl}_{2}(x)+2 \mathrm{Cl}_{2}(x-\pi)$. Many other properties can be found in [94] and the some are conveniently summarized in the appendix of [83].

We have used the notation of $\mathrm{Lu}[83]$, who used the hyperbolic Clausen function, $\mathrm{Clh}_{2}(x)$, and alternating hyperbolic Clausen function, $\mathrm{AClh}_{2}(a)$, defined by the integral representations

$$
\begin{aligned}
\mathrm{Clh}_{2}(x) & =-\int_{0}^{x} d y \log \left|2 \sinh \frac{y}{2}\right| \\
\mathrm{AClh}_{2}(x) & =-\int_{0}^{x} d y \log \left|2 \cosh \frac{y}{2}\right| .
\end{aligned}
$$

These can also be written as

$$
\begin{aligned}
\operatorname{Clh}_{2}(x) & =\frac{1}{2} \operatorname{Re}\left(\operatorname{Li}_{2}\left(e^{x}\right)-\operatorname{Li}_{2}\left(e^{-x}\right)\right) \\
\operatorname{AClh}_{2}(x) & =\frac{1}{2} \operatorname{Re}\left(\operatorname{Li}_{2}\left(-e^{x}\right)-\operatorname{Li}_{2}\left(-e^{-x}\right)\right)
\end{aligned}
$$

in analogy with Eq.(5.128).

Finally, some elementary relations which are used often include (for $x, y$ real)

$$
\begin{array}{r}
\arg (x+i y)=\tan ^{-1}\left(\frac{y}{x}\right)+\pi \theta(-x) \sigma(y) \\
\tan ^{-1}(x)+\tan ^{-1}(y)=\tan ^{-1}\left(\frac{x+y}{1-x y}\right)+\pi \sigma(x) \theta(x y-1) \\
\tan ^{-1}(x)+\tan ^{-1}(1 / x)=\sigma(x) \frac{\pi}{2} .
\end{array}
$$

where $\sigma(x)=x /|x|$ is the sign function and the step function $\theta(x)=(\sigma(x)+1) / 2$ 
should not be confused with the angle $\theta$.

\subsection{Appendix D : Form Factors with Supersym- metric Regularization}

Here we discuss the form factors regularized using dimensional reduction (DRED) in integer number of dimensions $d_{R}$, defined analogously to the usual $d_{R}=4$ DRED scheme. This could be used for $d_{R}=6$ or $d_{R}=10$ theories, but of course we mainly have in mind the four-dimensional case.

It is easy to see that the quark and scalar contributions are unchanged from DREG, and only the gluon contribution is different. This is most easily expressed in terms of the modified sum rule

$$
F_{G}(D R E D)+4 F_{Q}+\left(10-d_{R}\right) F_{S}=0
$$

which implies $F_{G}(D R E D)=F_{G}(D R E G)+\left(d_{R}-d\right) F_{S}$. Expanding $d=d_{R}-2 \epsilon$ around the real number of dimensions $d_{R}$ leads to $F_{G}(D R E D)=F_{G}(D R E G)+$ $2 \epsilon F_{S}$, which makes manifest the role of the $2 \epsilon$ adjoint DRED "ghosts" which preserve supersymmetry.

In four dimensions we have

$$
F_{G}(D R E D)+4 F_{Q}+6 F_{S}=0
$$

Since only the $A$ form factors have a UV divergence in four dimensions, only these form factors will be changed when using DRED:

$$
\delta_{D R E D}\left(A_{12}(G)\right)=\delta_{D R E D}\left(\bar{A}_{12}(G)\right)=-\frac{1}{3} \frac{i}{16 \pi^{2}} .
$$

In the symmetrized physical \pm basis we have $\delta_{D R E D}\left(A_{0}(G)\right)=-\frac{1}{3} \frac{i}{16 \pi^{2}}$, and all other form factors are unchanged. 


\subsection{Appendix E : Quark and Squark Mass Cor- rections}

Here the corrections to the form factors due to fermion and scalar masses will be given. The massive quark (MQ) contributions were first obtained in [84], and we obtain exactly the same results. To our knowledge, the squark contributions, either massless or massive (MS), have not yet appeared in the literature.

First, the well known formulas for the scalar and fermion self-energies are reproduced in our notation :

$$
\begin{aligned}
& \Pi_{1}(M Q)=\frac{d-2}{1-d} J_{1 M}+2 M^{2}\left(\frac{2 J_{1 M}-(d-2) T_{M}}{a(1-d)}\right) \\
& \Pi_{1}(M S)=\frac{1}{2(1-d)} J_{1 M}-M^{2}\left(\frac{2 J_{1 M}-(d-2) T_{M}}{a(1-d)}\right)
\end{aligned}
$$

with the integrals $J_{1 M}, T_{M}$ given in Appendix A. These yield the massive fermion and scalar contributions to the longitudinal form factors through Eq.(6.22).

Notice that the terms proportional to $M^{2}$ in the above two equations are equal up to a factor of -2 . After explicit calculation, it was discovered that the scalar mass correction terms $\left(\delta_{M S}\right)$ are just minus one-half of the quark mass correction terms $\left(\delta_{M Q}\right)$ for all form factors. Thus, for generic form factor $F$

$$
\begin{aligned}
F(M S) & =\left.F(S)\right|_{M}+\delta_{M S}(F) \\
F(M Q) & =\left.F(Q)\right|_{M}+\delta_{M Q}(F) \\
\delta_{M Q} & =-2 \delta_{M S}
\end{aligned}
$$

The notation $\left.F(S)\right|_{M}$ simply means to take the appropriate massless result for the form factor $F$, as given in section 3 , and replace $J \rightarrow J_{M}, J_{1} \rightarrow J_{1 M}, J_{2} \rightarrow J_{2 M}$, $J_{3} \rightarrow J_{3 M}$ everywhere.

Because of the relation $\delta_{M Q}=-2 \delta_{M S}$, we need only write either the fermion or scalar mass correction terms explicitly. Here we choose the scalar contributions, 
which for the transverse form factors are

$$
\begin{aligned}
\delta_{M S}\left(\bar{F}_{12}\right) & =-\frac{2 M^{2}}{\mathcal{K}^{2}}\left[\left(\frac{\mathcal{K}-3 \beta \gamma}{d-2}\right) J_{M}+\frac{\mathcal{P}-2 \alpha \mathcal{K}-\gamma^{2}(3 \alpha+2 \gamma-\beta)}{a(\beta-\gamma)(d-1)} J_{1 M}\right. \\
& \left.-\frac{\mathcal{P}-2 \alpha \mathcal{K}-\beta^{2}(3 \alpha+2 \beta-\gamma)}{b(\beta-\gamma)(d-1)} J_{2 M}-\frac{2 c}{d-1} J_{3 M}+\frac{(2-d) \mathcal{K} \alpha}{2(d-1) a b} T_{M}\right]
\end{aligned}
$$

and

$$
\delta_{M S}(\bar{H})=\frac{2 M^{2}}{\mathcal{K}^{2}}\left[\frac{3 \mathcal{P}}{d-2} J_{M}-\frac{\mathcal{K}-2 \alpha \gamma}{d-1} J_{1 M}-\frac{\mathcal{K}-2 \alpha \beta}{d-1} J_{2 M}-\frac{\mathcal{K}-2 \beta \gamma}{d-1} J_{3 M}+\frac{\mathcal{K}(d-2)}{2(d-1)} T_{M}\right]
$$

The results in the physical \pm basis can be obtained from those of the $L T$ basis through the use of Eq.(5.16), and are included here for completeness.

$$
\begin{aligned}
\delta_{M S}\left(A_{12}\right)=\frac{M^{2}}{\mathcal{K}}\left[\frac{c \alpha}{d-2} J_{M}+\frac{\gamma J_{1 M}+\beta J_{2 M}+c J_{3 M}}{d-1}\right] \\
\delta_{M S}\left(B_{12}\right)=-\frac{M^{2}}{\mathcal{K}}\left[\frac{(a-b) \alpha}{d-2} J_{M}+\frac{(2 \alpha+\gamma) J_{1 M}-(2 \alpha+\beta) J_{2 M}+(\beta-\gamma) J_{3 M}}{d-1}\right](5 \\
\delta_{M S}\left(C_{12}\right)= \\
+\frac{M^{2}}{4 \mathcal{K}^{2}}\left[\frac{8 \mathcal{P}+\alpha^{2} c+3 \beta^{2}(\alpha-\gamma)+3 \gamma^{2}(\alpha-\beta)}{d-2} J_{M}\right. \\
+\frac{8 \mathcal{P}+\alpha^{2}(\gamma-\beta)+\beta^{2}(5 \alpha+7 \gamma+4 \beta)}{b(d-1)} J_{2 M} \\
\left.+\frac{4 c^{2}+2 \beta \gamma-\mathcal{K}}{d-1} J_{3 M}+\frac{(d-2) \mathcal{K}\left(\alpha^{2}+2 \beta \gamma+3 \mathcal{K}\right)}{(d-1) 2 a b} T_{M}\right]
\end{aligned}
$$




$$
\begin{aligned}
\delta_{M S}\left(D_{12}\right) & =\frac{M^{2}}{4 \mathcal{K}^{2}}\left[\frac{\left(\mathcal{K}+3 \alpha^{2}\right)(a-b)}{d-2} J_{M}+\frac{\mathcal{K}+4 \alpha^{2}+2 \alpha \gamma}{d-1} J_{1 M}\right. \\
& \left.-\frac{\mathcal{K}+4 \alpha^{2}+2 \alpha \beta}{d-1} J_{2 M}+\frac{(2 \beta \gamma-3 \mathcal{K})(a-b)}{c(d-1)} J_{3 M}+\frac{(d-2) \mathcal{K}(a-b)}{2(d-1) c} T_{M}\right] \\
\delta_{M S}(H) & =\frac{M^{2}}{4 \mathcal{K}^{2}}\left[\frac{2 \mathcal{P}+a b c}{2-d} J_{M}+\frac{\mathcal{K}-2 \alpha \gamma}{d-1} J_{1 M}+\frac{\mathcal{K}-2 \alpha \beta}{d-1} J_{2 M}+\frac{\mathcal{K}-2 \beta \gamma}{d-1} J_{3 M}\right. \\
& \left.\left.-\frac{(d-2) \mathcal{K}}{2(d-1)} T_{M}\right)\right] \\
\delta_{M S}(S) & =-\frac{M^{2}}{4 \mathcal{K}^{2}}\left[\frac{3(a-b)(b-c)(c-a)}{2-d} J_{M}+\frac{\left(4 a^{2}+2 \alpha \gamma-3 \mathcal{K}\right)(b-c)}{a(d-1)} J_{1 M}\right. \\
& +\frac{\left(4 b^{2}+2 \alpha \beta-3 \mathcal{K}\right)(c-a)}{b(d-1)} J_{2 M}+\frac{\left(4 c^{2}+2 \beta \gamma-3 \mathcal{K}\right)(a-b)}{c(d-1)} J_{3 M} \\
& \left.\left.-\frac{(d-2)(a-b)(b-c)(c-a) \mathcal{K}}{2(d-1) a b c} T_{M}\right)\right] .
\end{aligned}
$$

It is straightforward to see that all of the correction terms are ultraviolet finite.

The relation $-2 \delta_{M S}=\delta_{M Q}$ is necessary for the preservation of the form of a quark/scalar sum $\Sigma_{S Q}=2 F_{S}+F_{Q}$, which is equal to $\frac{2}{d-10} \Sigma_{Q G}$ using the results of section 3. In other words $2 F_{M S}+F_{M Q}=\left.2 F_{S}\right|_{M}+\left.F_{Q}\right|_{M}$, so that this quantity has no correction terms proportional to $M^{2}$.

However, the relations between massive gauge bosons and massive fermions and/or scalars will be different, since the gauge bosons eat a degree of freedom to acquire mass. Consider the contribution of a massive gauge boson to the gauge-invariant gluon self-energy ${ }^{3}$ :

$$
\Pi_{1}(M G)=J_{1 M}\left[\frac{8 d-d_{R}-7}{2(d-1)}\right]+\frac{2 M^{2}}{a} \frac{\left(d_{R}-1\right)}{(d-1)}\left[J_{1 M}+\frac{1}{2}(2-d) T_{M}\right]
$$

\footnotetext{
${ }^{3}$ This is also the contribution of $W^{ \pm}$to the photon self-energy.
} 
where as before $d_{R}=d$ in dimensional regularization (DREG) and $d_{R}=4$ (or the real integer number of dimensions) in dimensional reduction (DRED). From this and Eq.(5.135) we deduce

$$
\Pi_{1}(M G)+4 \Pi_{1}(M Q)+\left(9-d_{R}\right) \Pi_{1}(M S)=0
$$

and thus the massive $N=4$ sum rule becomes

$$
F_{M G}+4 F_{M Q}+\left(9-d_{R}\right) F_{M S}=0
$$

for the longitudinal form factors. It can also be shown that this holds for the transverse form factors and so the results of this chapter also give the contributions of massive internal gauge bosons. Proving this involves detailed analysis of the vertices and diagrams that contribute to the triple gluon vertex when the PT/BFMFG is applied to a spontaneously broken gauge theory that leaves a non-abelian subgroup (of gluons) intact. This can be done following a pinch-technique route similar to [67][71]. Due to the equivalence of the PT and BFMFG, it is more convenient to follow the BFMFG route similar to [95]. For example, in $S U(5)$ GUTs the colored superheavy $X$ and $Y$ gauge bosons give a contribution which satisfies the above massive sum rule. We should emphasize that these sum rules are simply a convenient way of relating the contributions of various spin particles, all with hypothetical mass $M$, but no assumption is made about the actual masses for a given theory under consideration; the sum rules are entirely stripped of color factors. 


\section{Chapter 6}

\section{The Pinch-Technique Effective Charge at Two and Three-Loops}

\subsection{Introduction}

The conventional formulation of gauge theories leads to gauge-dependent Green's functions which are, by themselves, devoid of physical content even though the particular combinations which form physical quantities are gauge-invariant. For many purposes, however, it is desirable to have Green's functions which are gauge-invariant and physically meaningful by themselves. The two most well developed methods are the pinch technique (PT) and background field method (BFM). It was shown in [96] that the two methods were equivalent at one-loop. The two-loop PT gluon self-energy was explicitly constructed by Papavassiliou [36] through the rearrangement of the conventional two-loop corrections to a physical scattering process, and it was shown that the equivalence of the PT and BFM persisted at two loops. Recent progress has shown that the PT and BFM in quantum Feynman gauge (BFMFG) are equivalent to all orders in perturbation theory [37][38][39][76].

These special Green's functions have numerous positive attributes [34][31][32][64], including uniqueness, resummability of two-point functions, analyticity, unitarity, and a natural relation to optical theorem, from which they can also be derived [34][33][36]. 
Resumming these two-point functions leads to physical effective charges, ála Grunberg [11], which can be extended to the supersymmetric case and leads to an analytic improvement of gauge coupling unification with smooth threshold behavior [1]. Moreover, these Green's function can be renormalized independently and furnish a natural multi-scale analytic renormalization scheme, which was explored for the three-gluon vertex in [97].

The purpose of this chapter is to calculate the PT gluon self-energy to two-loops. This is interesting for several reasons. First, the finite parts will yield an improved numerical extraction of the $\mathrm{PT}$ effective charge from experimental data, as encoded in the $\overline{M S}$ coupling. Second, the three-loop $\beta$-function coefficient of the PT renormalization scheme is then easily derived. Third, the results here also give the longitudinal form factors of the gauge-invariant three-gluon vertex at two-loops. Finally, the status of a $\mathcal{N}=4$ supersymmetry sum rule can be investigated. In [97] (see also $[72,73,82]$ ), it was found that the triple-gluon vertex and the gluon self-energy of the PT were exactly zero in $\mathcal{N}=4$ theory at one-loop. Interestingly, at two-loops there remains a finite constant for $\mathcal{N}=4$ supersymmetry, as seen in Eq.(6.15).

\subsection{Notation and Setup}

The diagrams contributing to the PT two-loop gluon self-energy are shown in Figures 6.1, 6.2, and 6.3 in the Appendix. The results in Tables 6.1, 6.2, 6.3, 6.4, and 6.5 are given in terms of $A$ and $B$, which are related to the gluon self-energy by

$$
-i \Pi_{\mu \nu}^{a b(2-\mathrm{loop})}\left(p^{2}\right)=i g_{0}^{4} \delta^{a b}\left(\frac{-p^{2} e^{\gamma_{E}}}{4 \pi}\right)^{-2 \epsilon} \sum_{\text {diagrams }} \text { color } \times\left(A p^{2} g_{\mu \nu}-B p_{\mu} p_{\nu}\right) .
$$

The standard notation for the color factors is used, so that $C_{A} \equiv C_{2}(G)\left(=N_{c}\right.$ for $\left.S U\left(N_{c}\right)\right), \operatorname{Tr}\left[t_{f}^{a} t_{f}^{b}\right]=T_{F} \delta^{a b}, \operatorname{Tr}\left[t_{s}^{a} t_{s}^{b}\right]=T_{S} \delta^{a b}, t_{f}^{a} t_{f}^{a}=C_{F}$, and $t_{s}^{a} t_{s}^{a}=C_{S}$. The fermions are Dirac and the scalars are complex.

For the Yukawa graphs in Table 6.4, the notation of Machecek and Vaughn [46] (in Appendix A of paper I) has been used for the group theory factors $Y_{4}(F)$ and 
$Y_{4}(S)$ which are in the "color" column of Table 6.4. These are defined by

$$
\begin{aligned}
& Y_{4}(F)=\frac{1}{d(G)} \operatorname{Tr}\left[C(F) Y^{\alpha} Y^{\alpha \dagger}\right] \\
& Y_{4}(S)=\frac{1}{d(G)} \operatorname{Tr}\left[C(S) Y^{\alpha} Y^{\alpha \dagger}\right],
\end{aligned}
$$

and the Yukawa interactions are of the form $\mathcal{L}_{\text {int }}=-Y_{I J}^{\alpha} \phi_{\alpha} \psi_{I} \psi_{J}+$ h.c.. Since these contain two powers of coupling, the $g_{0}^{4}$ in the above formula (Eq.(6.1)) should be replaced by $g_{0}^{2}$. For the Yukawa graphs the fermions are Weyl and the scalars are real. Note that although the $Y_{4}(S)$ term does not contribute to the $\beta$ function [46], it does contribute finite parts which are important here. In this chapter we will only be interested in supersymmetric Yukawa couplings, which have the particular values (see Eq.(4.29))

$$
\begin{aligned}
& Y_{4}(F)=2 g^{2} T_{Y}\left(C_{Y}+C_{A}\right) \\
& Y_{4}(S)=4 g^{2} T_{Y} C_{Y} .
\end{aligned}
$$

Here $\operatorname{Tr}\left[t_{\Phi}^{a} t_{\Phi}^{b}\right]=T_{Y} \delta^{a b}$ and $t_{\Phi}^{a} t_{\Phi}^{a}=C_{Y}$ are just the usual trace and Casimir color factors for the chiral supermultiplet $(\Phi)$ to which the gaugino is coupled in the loops. These are labeled separately to take into account the possibility of multiple supersymmetric Yukawa vertices. For example, momentarily we will be interested in $\mathcal{N}=4$ supersymmetry, which has the values $T_{Y}=6 C_{A}$ and $C_{Y}=C_{A}$.

Of course the totals for each independent color structure must be transverse $(A=$ $B)$, which is a useful check. In [79][98] it was noted that the $\frac{1}{\epsilon^{2}}$ pole terms were transverse graph by graph. Here we find that the finite $\pi^{2}$ and $\zeta_{3}$ terms are also transverse graph by graph.

The results for both dimensional regularization (DREG) and dimensional reduction (DRED) are given through the use of the parameter $\delta_{R}$ [99]. This appears in the dimension $d=4-2 \epsilon \delta_{R}$ when performing internal metric contractions, which are treated differently in the two schemes. The values 0 and 1 correspond to DRED and DREG, respectively. The results of Tables $6.1,6.2,6.3$, and 6.4 are the results for DREG, while Table 6.5 gives the terms proportional to $\left(1-\delta_{R}\right)$, i.e. the correction 
terms for DRED.

The divergent parts of the BFMFG gluon self-energy were first given in [79] for the pure gluon and fermion contributions. The divergent parts of the full quantumgauge-parameter dependent results for the pure-gluon case at two-loops were given in [98]. Finally, the finite terms which depended on the difference between $\overline{M S}$ and $\overline{D R}$ schemes $\left(\delta_{R}\right)$ were calculated in [99]. The results of this chapter are in complete agreement with these previous calculations. The new results presented here are the finite parts of all of the graphs, including gluons, fermions, scalars, and Yukawa couplings, all given in the massless limit.

\subsection{Results and Applications}

\subsubsection{The effective charge and relation to $\overline{M S}$}

The gauge-invariant gluon self-energy derived from the PT/BFMFG is used to define the pinch-technique effective charge through the relation

$$
\hat{\alpha}\left(p^{2}\right) \equiv \frac{\alpha_{0}}{1+\hat{\Pi}\left(p^{2}\right)}
$$

where the bare (dimensionful) coupling is $\alpha_{0}=g_{0}^{2} /(4 \pi)$ and we expand through two loops

$$
\hat{\Pi}\left(p^{2}\right)=\hat{\Pi}_{1}\left(p^{2}\right)+\hat{\Pi}_{2}\left(p^{2}\right)+\cdots .
$$

Defining the bare, $\overline{M S}$, and PT couplants by

$$
a_{0}=\frac{\alpha_{0}}{4 \pi} \quad \bar{a}=\frac{\bar{\alpha}}{4 \pi} \quad \hat{a}=\frac{\hat{\alpha}}{4 \pi}
$$

the results this chapter can be written as

$$
\begin{aligned}
& \hat{\Pi}_{1}\left(p^{2}\right)=a_{0} e^{-\epsilon L_{p}}\left(-\frac{\beta_{0}}{\epsilon}+C_{1}\right) \\
& \hat{\Pi}_{2}\left(p^{2}\right)=a_{0}^{2} e^{-2 \epsilon L_{p}}\left(-\frac{\beta_{1}}{2 \epsilon}+C_{2}\right)
\end{aligned}
$$


where $L_{p}=\log \left(-p^{2}\right)+\gamma_{E}-\log 4 \pi$. The first two $\beta$ function coefficients are

$$
\begin{aligned}
& \beta_{0}=\frac{11}{3} C_{A}-\frac{4}{3} T_{F}-\frac{1}{3} T_{S} \\
& \beta_{1}=\frac{34}{3} C_{A}^{2}-T_{F}\left(\frac{20}{3} C_{A}+4 C_{F}\right)-T_{S}\left(\frac{2}{3} C_{A}+4 C_{S}\right)+\frac{1}{g^{2}} Y_{4}(F)
\end{aligned}
$$

and the finite terms are

$$
\begin{aligned}
C_{1} & =\left(-\frac{64}{9}-\frac{1}{3} \delta_{R}\right) C_{A}+\frac{20}{9} T_{F}+\frac{8}{9} T_{S} \\
C_{2} & =\left(6 \zeta_{3}-\frac{659}{18}-\frac{7}{3} \delta_{R}\right) C_{A}^{2}+\left(8 \zeta_{3}+\frac{158}{9}\right) C_{A} T_{F}+\left(-16 \zeta_{3}+\frac{49}{3}+2 \delta_{R}\right) C_{F} T_{F} \\
& +\left(3 \zeta_{3}+\frac{43}{18}\right) C_{A} T_{S}+\left(-4 \zeta_{3}+\frac{43}{3}\right) C_{S} T_{S}-\frac{1}{4 g^{2}}\left(3 Y_{4}(S)+11 Y_{4}(F)\right)
\end{aligned}
$$

The two-loop constant term $C_{2}$ is a new result, while the $C_{1}$ term and of course $\beta_{0}$ and $\beta_{1}$ are known.

In order to extract the numerical value of the PT coupling we have to compare to the data for some physical observable(s). Since most of the relevant data has been incorporated into the $\overline{M S}$ coupling $\left(\bar{\alpha}\left(\mu^{2}\right)\right)$ over the years, the relation between $\hat{\alpha}$ and $\bar{\alpha}$ should be used. In the convention where the bare coupling is dimensionful, the bare and $\overline{M S}$ couplants are related by

$$
a_{0}=\bar{a}\left(\mu^{2}\right) e^{\epsilon \bar{L}}\left[1-\bar{a}\left(\mu^{2}\right) \frac{\beta_{0}}{\epsilon}+\bar{a}^{2}\left(\mu^{2}\right)\left(\frac{\beta_{0}^{2}}{\epsilon^{2}}-\frac{\beta_{1}}{2 \epsilon}\right)\right]
$$

where $\bar{L}=\log \mu^{2}+\gamma_{E}-\log 4 \pi$. Substituting this into Eq.(6.4) leads to

$$
\begin{aligned}
\frac{1}{\hat{a}\left(p^{2}\right)} & =\frac{1}{\bar{a}\left(\mu^{2}\right)}+\beta_{0} \log \left(\frac{-p^{2}}{\mu^{2}}\right)+C_{1}+\bar{a}\left(\mu^{2}\right)\left[\beta_{1} \log \left(\frac{-p^{2}}{\mu^{2}}\right)+C_{2}\right] \\
\frac{1}{\hat{a}\left(-Q^{2}\right)} & =\frac{1}{\bar{a}\left(Q^{2}\right)}+C_{1}+\bar{a}\left(Q^{2}\right) C_{2} .
\end{aligned}
$$

Comparing with $\overline{M S}$ value $\bar{\alpha}\left(M_{Z}^{2}\right)=0.118 \pm 0.003$ and using the proper QCD values of the color factors at scale $M_{Z}\left(C_{A}=3, T_{F}=N_{F} / 2=5 / 2, C_{F}=4 / 3, T_{S}=C_{S}=\right.$ 
$\left.T_{Y}=C_{Y}=0\right)$, we find

$$
\begin{array}{ll}
\hat{\alpha}\left(-M_{Z}^{2}\right)=0.1413 \pm 0.0043 & 2 \text { loops } \\
\hat{\alpha}\left(-M_{Z}^{2}\right)=0.1401 \pm 0.0042 & 1 \text { loop }
\end{array}
$$

The small upward shift from the two-loop corrections is in very good agreement with predictions using BLM methods (see Chapter 8 ). The BLM predictions for the constant $C_{2}$ (in $\mathrm{QCD}$ ) is within $10 \%$ of the correct value.

Eq.(6.12) was obtained using $\delta_{R}=1$, appropriate for DREG and $\overline{M S}$. The same results would be obtained by relating the PT coupling to the $\overrightarrow{D R}$ coupling, with $\delta_{R}=0$. In fact, the relation [99] between the $\overline{M S}$ and $\overline{D R}$ couplings can be obtained by requiring that the value of the PT coupling does not depend on the choice of regularization.

\subsection{2 $\mathcal{N}=4$ supersymmetric gauge theory}

For $\mathcal{N}=4$ supersymmetry, the supersymmetric values for the Yukawa invariants given in Eq.(6.3) should be used in Eq.(6.9), along with the following values for the color factors :

$$
\begin{aligned}
C_{F} & =C_{S}=C_{Y}=C_{A} \\
T_{F} & =4\left(\frac{1}{2} C_{A}\right)=2 C_{A} \\
T_{S} & =6\left(\frac{1}{2} C_{A}\right)=3 C_{A} \\
T_{Y} & =6 C_{A}
\end{aligned}
$$

This leads to

$$
\begin{aligned}
& \hat{\Pi}_{1}=a_{0} \frac{\delta_{R}}{9} C_{A} \\
& \hat{\Pi}_{2}=a_{0}^{2}\left(13 \zeta_{3}+\frac{8}{3}-\frac{5}{3} \delta_{R}\right) C_{A}^{2} .
\end{aligned}
$$


Using the supersymmetric regulator DRED $\left(\delta_{R}=0\right)$ yields $\hat{\Pi}_{1}=0$ but $\hat{\Pi}_{2} \neq 0$. Thus, the $\mathcal{N}=4$ gauge supermultiplet gives a nonzero finite contribution at two-loops.

It should be noted that the Yukawa graphs do not have any $\zeta_{3}$ terms, which arise from only the master-type graphs $\mathrm{S} 3, \mathrm{~S} 4, \mathrm{~F} 4, \mathrm{G} 3$, and $\mathrm{H} 6$. Thus, it is only necessary to look at these graphs to see that in fact $\hat{\Pi}_{2}(\mathcal{N}=4) \neq 0$.

\subsubsection{Three-loop $\beta$ function}

The definitions of the $\beta$ functions for the $P T$ and $\overline{M S}$ couplings,

$$
\begin{aligned}
& \hat{\beta}=\frac{\partial \hat{a}}{\partial \log q^{2}}=-\hat{a}^{2}\left[\beta_{0}+\beta_{1} \hat{a}+\hat{\beta}_{2} \hat{a}^{2}+\cdots\right] \\
& \bar{\beta}=\frac{\partial \bar{a}}{\partial \log q^{2}}=-\bar{a}^{2}\left[\beta_{0}+\beta_{1} \bar{a}+\bar{\beta}_{2} \bar{a}^{2}+\cdots\right],
\end{aligned}
$$

combined with Eq.(6.11) leads to

$$
\hat{\beta}_{2}=\bar{\beta}_{2}+C_{1} \beta_{1}-C_{2} \beta_{0} .
$$

Using the known $[100,101]$ results for the three-loop $\beta$-function coefficient of QCD in the $\overline{M S}$ scheme

$$
\bar{\beta}_{2}=\frac{2857}{54} C_{A}^{3}-\frac{1415}{27} C_{A}^{2} T_{F}-\frac{205}{9} C_{A} C_{F} T_{F}+\frac{158}{27} C_{A} T_{F}^{2}+\frac{44}{9} C_{F} T_{F}^{2}+2 C_{F}^{2} T_{F}
$$

we obtain the three-loop $\beta$-function coefficient of the $P T$ scheme in QCD

$$
\begin{aligned}
\hat{\beta}_{2} & =\left(\frac{334}{3}-22 \zeta_{3}\right) C_{A}^{3}-\left(\frac{845}{9}+\frac{64}{3} \zeta_{3}\right) C_{A}^{2} T_{F}-\left(\frac{542}{9}-\frac{176}{3} \zeta_{3}\right) C_{A} C_{F} T_{F} \\
& +\left(\frac{130}{9}+\frac{32}{3} \zeta_{3}\right) C_{A} T_{F}^{2}+\left(\frac{184}{9}-\frac{64}{3} \zeta_{3}\right) C_{F} T_{F}^{2}+2 C_{F}^{2} T_{F}
\end{aligned}
$$

It is interesting to compare to another effective charge, $\alpha_{V}$, defined from the heavy quark potential [102], even though use of this charge has fallen out of favor due to its infrared problems. Nevertheless, both the PT and the $V$-scheme share the philosophy of trying to construct physically meaningful Green's functions that 
measures the actual strength of the forces. The two-loop finite terms and three-loop $\beta$ function of the $V$-scheme were calculated in [103].

Numerically we find

$$
\begin{aligned}
& \hat{\beta}_{2} \approx 2292-516 N_{F}+18.7 N_{F}^{2} \\
& \bar{\beta}_{2} \approx 1429-280 N_{F}+6.0 N_{F}^{2} \\
& \beta_{2}^{V} \approx 4224-746 N_{F}+20.9 N_{F}^{2}
\end{aligned}
$$

and

\begin{tabular}{|c|c|c|c|}
\hline$N_{F}$ & $\bar{\beta}_{2}$ & $\hat{\beta}_{2}$ & $\beta_{2}^{V}$ \\
\hline 3 & 644 & 914 & 2174 \\
4 & 406 & 529 & 1574 \\
5 & 181 & 182 & 1016 \\
6 & -33 & -127 & 500 \\
\hline
\end{tabular}

It is interesting the $\mathrm{PT}$ and $\overline{M S}$ values are numerically similar in comparison to the $V$-scheme, which has a rather large $\beta_{2}$.

\subsubsection{The longitudinal form factors of the gauge-invariant three-gluon vertex}

In Chapter 5, the form factors of the gauge-invariant three-gluon vertex [60] are given at one loop. An all-orders relation between the gauge-invariant gluon three- and two-point functions is encoded in the Ward identity

$$
p_{3}^{\mu_{3}} \Gamma_{\mu_{1} \mu_{2} \mu_{3}}\left(p_{1}, p_{2}, p_{3}\right)=t_{\mu_{1} \mu_{2}}\left(p_{2}\right)\left(1+\Pi\left(p_{2}^{2}\right)\right)-t_{\mu_{1} \mu_{2}}\left(p_{1}\right)\left(1+\Pi\left(p_{1}^{2}\right)\right) .
$$


which can be solved for the ten longitudinal form factors, yielding

$$
\begin{aligned}
\bar{A}_{12} & =\frac{\hat{\Pi}\left(p_{1}^{2}\right)+\hat{\Pi}\left(p_{2}^{2}\right)}{2} \\
\bar{B}_{12} & =\frac{\hat{\Pi}\left(p_{1}^{2}\right)-\hat{\Pi}\left(p_{2}^{2}\right)}{2} \\
\bar{C}_{12} & =\frac{\hat{\Pi}\left(p_{1}^{2}\right)-\hat{\Pi}\left(p_{2}^{2}\right)}{p_{1}^{2}-p_{2}^{2}} \\
\bar{S} & =0,
\end{aligned}
$$

and cyclic permutations $\bar{A}_{23}, \bar{A}_{31}, \bar{B}_{23}, \bar{B}_{31}, \bar{C}_{23}, \bar{C}_{31}$. The bars on the form factors simply denote the $L T$ basis [97] and have nothing to do with $\overline{M S}$. The results of section 6.3.1 combined with Eq.(6.22) lead to the two-loop longitudinal form factors.

Acknowledgements The author would like to thank Lance Dixon for providing a check on some of the diagrams. 


\subsection{Appendix : Results for Diagrams}

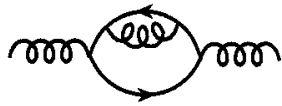

F2

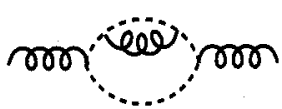

S2

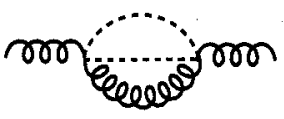

S5
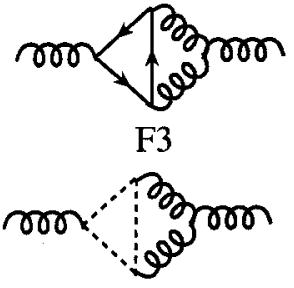

S3

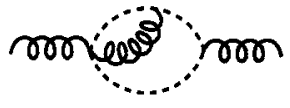

S6

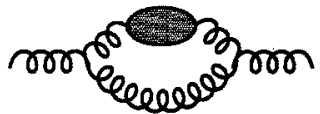

$\mathrm{F} 1, \mathrm{~S} 1, \mathrm{G} 1, \mathrm{H} 1$
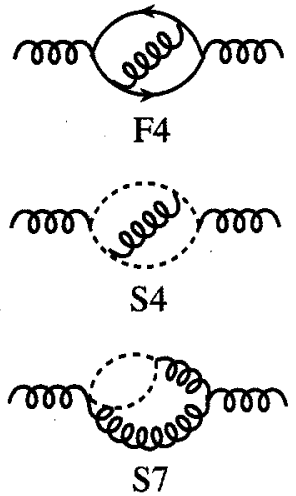

Figure 6.1: The Diagrams with fermions (F) and scalars (S). The blob in the bottom diagram represents the one-loop quantum gluon self-energy insertion. The diagrams implicitly include any mirror image diagrams which are not shown, so that S6 represents 4 diagrams, while F3 represents two diagrams, etc. 


\begin{tabular}{|c|c|c|c|}
\hline Diagram & color $\left(\times T_{F}\right)$ & $A$ & $B$ \\
\hline$F 1$ & $C_{A}$ & $-\frac{839}{18}+\frac{5}{9} \pi^{2}-\frac{13}{\epsilon}-\frac{10}{3} \frac{1}{\epsilon^{2}}$ & $-\frac{436}{9}+\frac{5}{9} \pi^{2}-\frac{40}{3} \frac{1}{\epsilon}-\frac{10}{3} \frac{1}{\epsilon^{2}}$ \\
$F 2$ & $C_{F}$ & $\frac{56}{3}-\frac{2}{9} \pi^{2}+\frac{16}{3} \frac{1}{\epsilon}+\frac{4}{3} \frac{1}{\epsilon^{2}}$ & $15-\frac{2}{9} \pi^{2}+\frac{14}{3} \frac{1}{\epsilon}+\frac{4}{3} \frac{1}{\epsilon^{2}}$ \\
$F 3$ & $C_{A}$ & $\frac{61}{3}-\frac{4}{9} \pi^{2}+\frac{26}{3} \frac{1}{\epsilon}+\frac{8}{3} \frac{1}{\epsilon^{2}}$ & $24-\frac{4}{9} \pi^{2}+\frac{28}{3} \frac{1}{\epsilon}+\frac{8}{3} \frac{1}{\epsilon^{2}}$ \\
$F 4$ & $C_{F}-\frac{1}{2} C_{A}$ & $-37+\frac{2}{9} \pi^{2}+16 \zeta_{3}-\frac{22}{3} \frac{1}{\epsilon}-\frac{4}{3} \frac{1}{\epsilon^{2}}$ & $-\frac{100}{3}+\frac{2}{9} \pi^{2}+16 \zeta_{3}-\frac{20}{3} \frac{1}{\epsilon}-\frac{4}{3} \frac{1}{\epsilon^{2}}$ \\
$R F 1$ & $C_{A}$ & $-\frac{2008}{81}+\frac{5}{27} \pi^{2}-\frac{284}{27} \frac{1}{\epsilon}-\frac{20}{9} \frac{1}{\epsilon^{2}}$ & $-\frac{2008}{81}+\frac{5}{27} \pi^{2}-\frac{284}{27} \frac{1}{\epsilon}-\frac{20}{9} \frac{1}{\epsilon^{2}}$ \\
$R F 2$ & $C_{A}$ & $\frac{1216}{81}-\frac{5}{27} \pi^{2}+\frac{212}{27} \frac{1}{\epsilon}+\frac{20}{9} \frac{1}{\epsilon^{2}}$ & $\frac{1216}{81}-\frac{5}{27} \pi^{2}+\frac{212}{27} \frac{1}{\epsilon}+\frac{20}{9} \frac{1}{\epsilon^{2}}$ \\
\hline
\end{tabular}

Table 6.1: Diagrams with Fermions

\begin{tabular}{|c|c|c|c|}
\hline Diagram & color $\left(\times T_{S}\right)$ & $A$ & $B$ \\
\hline$S 1$ & $C_{A}$ & $-\frac{1133}{72}+\frac{5}{36} \pi^{2}-\frac{49}{12} \frac{1}{\epsilon}-\frac{5}{6} \frac{1}{\epsilon^{2}}$ & $-\frac{293}{18}+\frac{5}{36} \pi^{2}-\frac{25}{6} \frac{1}{\epsilon}-\frac{5}{6} \frac{1}{\epsilon^{2}}$ \\
$S 2$ & $C_{S}$ & $-\frac{115}{6}+\frac{1}{9} \pi^{2}-\frac{13}{3} \frac{1}{\epsilon}-\frac{2}{3} \frac{1}{\epsilon^{2}}$ & $-17+\frac{1}{9} \pi^{2}-\frac{4}{\epsilon}-\frac{2}{3} \frac{1}{\epsilon^{2}}$ \\
$S 3$ & $C_{A}$ & $\frac{213}{16}-\frac{11}{72} \pi^{2}-\zeta_{3}+\frac{101}{24} \frac{1}{\epsilon}+\frac{11}{12} \frac{1}{\epsilon^{2}}$ & $\frac{691}{48}-\frac{11}{72} \pi^{2}-\zeta_{3}+\frac{35}{8} \frac{1}{\epsilon}+\frac{11}{12} \frac{1}{\epsilon^{2}}$ \\
$S 4$ & $\left(C_{S}-\frac{1}{2} C_{A}\right)$ & $-\frac{209}{12}+\frac{1}{18} \pi^{2}+4 \zeta_{3}-\frac{19}{6} \frac{1}{\epsilon}-\frac{1}{3} \frac{1}{\epsilon^{2}}$ & $-\frac{157}{12}+\frac{1}{18} \pi^{2}+4 \zeta_{3}-\frac{5}{2} \frac{1}{\epsilon}-\frac{1}{3} \frac{1}{\epsilon^{2}}$ \\
$S 5$ & $\left(C_{A}-4 C_{S}\right)$ & $\frac{13}{8}+\frac{1}{4} \frac{1}{\epsilon}$ & 0 \\
$S 6$ & $\left(2 C_{S}-\frac{1}{2} C_{A}\right)$ & $\frac{115}{8}-\frac{1}{12} \pi^{2}+\frac{13}{4} \frac{1}{\epsilon}+\frac{1}{2} \frac{1}{\epsilon^{2}}$ & $\frac{63}{8}-\frac{1}{12} \pi^{2}+\frac{9}{4} \frac{1}{\epsilon}+\frac{1}{2} \frac{1}{\epsilon^{2}}$ \\
$R S 1$ & $C_{A}$ & $-\frac{640}{81}+\frac{5}{108} \pi^{2}-\frac{86}{27} \frac{1}{\epsilon}-\frac{5}{9} \frac{1}{\epsilon^{2}}$ & $-\frac{640}{81}+\frac{5}{108} \pi^{2}-\frac{86}{27} \frac{1}{\epsilon}-\frac{5}{9} \frac{1}{\epsilon^{2}}$ \\
$R S 2$ & $C_{A}$ & $\frac{388}{81}-\frac{5}{108} \pi^{2}+\frac{68}{27} \frac{1}{\epsilon}+\frac{5}{9} \frac{1}{\epsilon^{2}}$ & $\frac{388}{81}-\frac{5}{108} \pi^{2}+\frac{68}{27} \frac{1}{\epsilon}+\frac{5}{9} \frac{1}{\epsilon^{2}}$ \\
\hline \multicolumn{4}{|c|}{} \\
\hline \multicolumn{2}{|c|}{ Total $(A=B)$} & $T_{S} C_{S}\left(-\frac{43}{3}+4 \zeta_{3}-\frac{2}{\epsilon}\right)+T_{S} C_{A}\left(-\frac{43}{18}-3 \zeta_{3}-\frac{1}{3} \frac{1}{\epsilon}\right)$ \\
\hline
\end{tabular}

Table 6.2: Diagrams with Scalars 


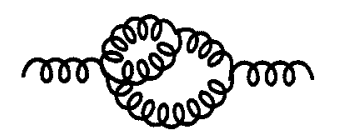

G2

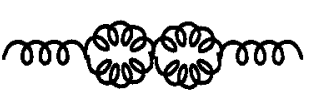

G5

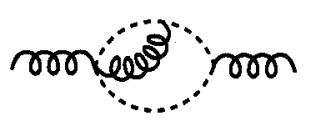

$\mathrm{H} 4$

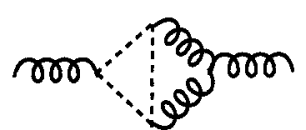

$\mathrm{H} 7$

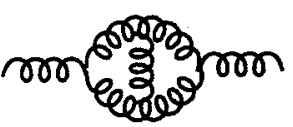

G3
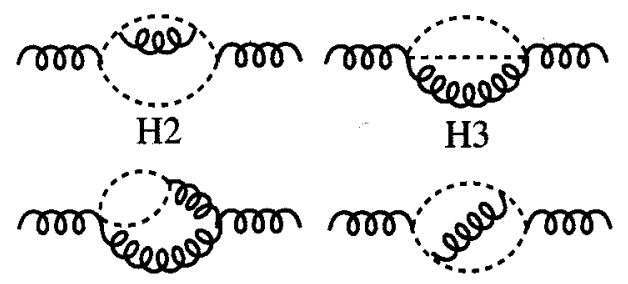

H5

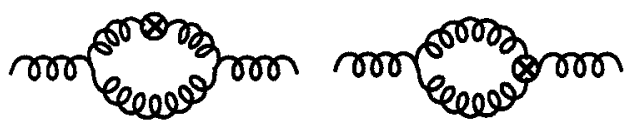

RF1, RS1, RGH1 RF2, RS2, RGH2

Figure 6.2: The Diagrams with only gluons $(\mathrm{G})$ and ghosts $(\mathrm{H})$. The $\otimes$ in the bottom two diagrams represents the gauge-fixing-parameter counterterm insertions.

\begin{tabular}{|c|c|c|}
\hline Diagram & $A$ & $B$ \\
\hline$G H 1$ & $\frac{4783}{72}-\frac{25}{36} \pi^{2}+\frac{215}{12} \frac{1}{\epsilon}+\frac{25}{6} \frac{1}{\epsilon^{2}}$ & $\frac{620}{9}-\frac{25}{36} \pi^{2}+\frac{55}{3} \frac{1}{\epsilon}+\frac{25}{6} \frac{1}{\epsilon^{2}}$ \\
$G 2$ & $-\frac{255}{32}+\frac{3}{16} \pi^{2}-\frac{57}{16} \frac{1}{\epsilon}-\frac{9}{8} \frac{1}{\epsilon^{2}}$ & $-\frac{675}{32}+\frac{3}{16} \pi^{2}-\frac{93}{16} \frac{1}{\epsilon}-\frac{9}{8} \frac{1}{\epsilon^{2}}$ \\
$G 3$ & $\frac{4673}{96}-\frac{9}{16} \pi^{2}-\frac{11}{2} \zeta_{3}+\frac{233}{16} \frac{1}{\epsilon}+\frac{27}{8} \frac{1}{\epsilon^{2}}$ & $\frac{5093}{96}-\frac{9}{16} \pi^{2}-\frac{11}{2} \zeta_{3}+\frac{245}{16} \frac{1}{\epsilon}+\frac{27}{8} \frac{1}{\epsilon^{2}}$ \\
$G 4$ & $-\frac{105}{16}-\frac{9}{6} \frac{1}{\epsilon}$ & 0 \\
$G 5$ & $-72+\pi^{2}-\frac{24}{\epsilon}-\frac{6}{\epsilon^{2}}$ & $-72+\pi^{2}-\frac{24}{\epsilon}-\frac{6}{\epsilon^{2}}$ \\
$H 2$ & $\frac{115}{24}-\frac{1}{36} \pi^{2}+\frac{13}{12} \frac{1}{\epsilon}+\frac{1}{6} \frac{1}{\epsilon^{2}}$ & $\frac{17}{4}-\frac{1}{36} \pi^{2}+\frac{1}{\epsilon}+\frac{1}{6} \frac{1}{\epsilon^{2}}$ \\
$H 3$ & $\frac{13}{16}+\frac{1}{8} \frac{1}{\epsilon}$ & 0 \\
$H 4$ & $-\frac{115}{24}+\frac{1}{36} \pi^{2}-\frac{13}{12} \frac{1}{\epsilon}-\frac{1}{6} \frac{1}{\epsilon^{2}}$ & $-\frac{21}{8}+\frac{1}{36} \pi^{2}-\frac{3}{4} \frac{1}{\epsilon}-\frac{1}{6} \frac{1}{\epsilon^{2}}$ \\
$H 5$ & $-\frac{263}{96}+\frac{5}{144} \pi^{2}-\frac{41}{48} \frac{1}{\epsilon}-\frac{5}{24} \frac{1}{\epsilon^{2}}$ & $-\frac{105}{32}+\frac{5}{144} \pi^{2}-\frac{15}{16} \frac{1}{\epsilon}-\frac{5}{24} \frac{1}{\epsilon^{2}}$ \\
$H 6$ & $\frac{209}{96}-\frac{1}{144} \pi^{2}-\frac{1}{2} \zeta_{3}+\frac{19}{48} \frac{1}{\epsilon}+\frac{1}{24} \frac{1}{\epsilon^{2}}$ & $\frac{157}{96}-\frac{1}{144} \pi^{2}-\frac{1}{2} \zeta_{3}+\frac{5}{16} \frac{1}{\epsilon}+\frac{1}{24} \frac{1}{\epsilon^{2}}$ \\
$H 7$ & $-\frac{55}{16}+\frac{1}{24} \pi^{2}-\frac{9}{8} \frac{1}{\epsilon}-\frac{1}{4} \frac{1}{\epsilon^{2}}$ & $-\frac{55}{16}+\frac{1}{24} \pi^{2}-\frac{9}{8} \frac{1}{\epsilon}-\frac{1}{4} \frac{1}{\epsilon^{2}}$ \\
$R G H 1$ & $\frac{2786}{81}-\frac{25}{108} \pi^{2}+\frac{385}{27} \frac{1}{\epsilon}+\frac{25}{9} \frac{1}{\epsilon^{2}}$ & $\frac{2786}{81}-\frac{25}{108} \pi^{2}+\frac{385}{27} \frac{1}{\epsilon}+\frac{25}{9} \frac{1}{\epsilon^{2}}$ \\
$R G H 2$ & $-\frac{1688}{81}+\frac{25}{108} \pi^{2}-\frac{295}{27} \frac{1}{\epsilon}-\frac{25}{9} \frac{1}{\epsilon^{2}}$ & $-\frac{1688}{81}+\frac{25}{108} \pi^{2}-\frac{295}{27} \frac{1}{\epsilon}-\frac{25}{9} \frac{1}{\epsilon^{2}}$ \\
\hline
\end{tabular}

\begin{tabular}{|l|l|}
\hline $\operatorname{Total}(A=B)$ & $C_{A}^{2}\left(\frac{701}{18}-6 \zeta_{3}+\frac{17}{3} \frac{1}{\epsilon}\right)$ \\
\hline
\end{tabular}

Table 6.3: Diagrams with only gluons and ghosts. Each term has color factor $C_{A}^{2}$. 


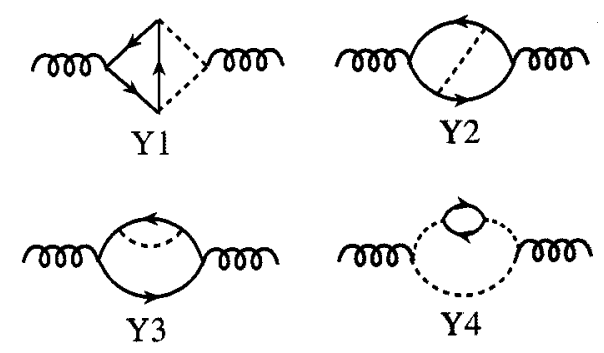

Figure 6.3: The Yukawa Diagrams $(\mathrm{Y})$.

\begin{tabular}{|c|c|c|c|}
\hline Diagram & color $\left(\times T_{F}\right)$ & $A$ & $B$ \\
\hline$Y 1$ & $Y_{4}(S)$ & $-\frac{35}{6}+\frac{1}{18} \pi^{2}-\frac{5}{3} \frac{1}{\epsilon}-\frac{1}{3} \frac{1}{\epsilon^{2}}$ & $-\frac{19}{4}+\frac{1}{18} \pi^{2}-\frac{3}{2} \frac{1}{\epsilon}-\frac{1}{3} \frac{1}{\epsilon^{2}}$ \\
$Y 2$ & $Y_{4}(F)-\frac{1}{2} Y_{4}(S)$ & $-\frac{43}{12}+\frac{1}{18} \pi^{2}-\frac{7}{6} \frac{1}{\epsilon}-\frac{1}{3} \frac{1}{\epsilon^{2}}$ & $-\frac{5}{2}+\frac{1}{18} \pi^{2}-\frac{1}{\epsilon}-\frac{1}{3} \frac{1}{\epsilon^{2}}$ \\
$Y 3$ & $Y_{4}(F)$ & $\frac{19}{3}-\frac{1}{18} \pi^{2}+\frac{5}{3} \frac{1}{\epsilon}+\frac{1}{3} \frac{1}{\epsilon^{2}}$ & $\frac{21}{4}-\frac{1}{18} \pi^{2}+\frac{3}{2} \frac{1}{\epsilon}+\frac{1}{3} \frac{1}{\epsilon^{2}}$ \\
$Y 4$ & $Y_{4}(S)$ & $\frac{115}{24}-\frac{1}{36} \pi^{2}+\frac{13}{12} \frac{1}{\epsilon}+\frac{1}{6} \frac{1}{\epsilon^{2}}$ & $\frac{17}{4}-\frac{1}{36} \pi^{2}+\frac{1}{\epsilon}+\frac{1}{6} \frac{1}{\epsilon^{2}}$ \\
\hline
\end{tabular}

\begin{tabular}{|l|l|}
\hline $\operatorname{Total}(A=B)$ & $\frac{3}{4} Y_{4}(S)+\left(\frac{11}{4}+\frac{1}{2 \epsilon}\right) Y_{4}(F)$ \\
\hline
\end{tabular}

Table 6.4: Diagrams with Yukawa couplings

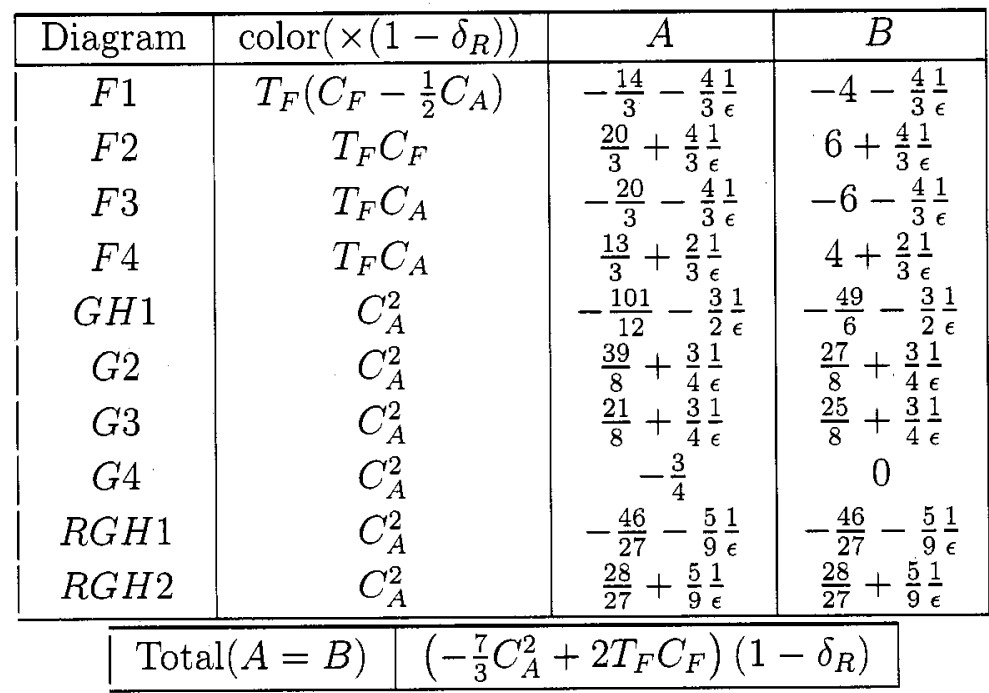

Table 6.5: Corrections due to Supersymmetric Regularization. $\delta_{R}=0$ in DRED, and $\delta_{R}=1$ in DREG. 


\section{Chapter 7}

\section{The Higgs Boson Mass in Split Supersymmetry at Two-Loops}

\section{$7.1 \quad$ Introduction}

The gauge hierarchy problem of the Standard Model (SM) of particle physics has been a fruitful source of inspiration for beyond the SM physics. Most notably, a main reason for the prominence of supersymmetry was its natural solution to this problem. In recent years additional circumstantial evidence for supersymmetry (SUSY) has arisen from gauge coupling unification and from dark matter, although these successes have been partially offset by difficulties with flavor changing neutral currents and $\mathrm{CP}$ violation which arise from light SUSY scalars. Thus, it may be reasonable to abandon the original motivation for SUSY and consider the implications of a theory which maintains all of the successes of the MSSM, except for the hierarchy problem, and does away with some of the difficulties. This proposal, called finely tuned, or split supersymmetry, has appeared in [57][58], and some phenomenology has been discussed $[104,105,106,107,108,109,110]$. In split supersymmetry, a single Higgs scalar is fine tuned to be light, with the understanding that the fine tuning will be resolved by some anthropic-like selection effects. This approach may have a natural realization within inflation and string theory [111], where an almost infinite landscape 
of vacua may contain a small percentage which have the desired fine-tuned parameters necessary for life and the properties of our universe.

The prediction for the Higgs boson mass is typically higher in Split SUSY than MSSM scenarios, and is thus a key distinguishing feature. The MSSM Higgs mass is known to two-loop accuracy [112]. The purpose of this chapter is to bring the split supersymmetry Higgs mass prediction to a similar level of precision.

\subsection{Corrections to the Higgs Mass}

The starting point for our analysis is the split SUSY Lagrangian [57][58]

$$
\begin{aligned}
\mathcal{L} & =m^{2} H^{\dagger} H-\frac{\lambda}{2}\left(H^{\dagger} H\right)^{2}+F_{U} \hat{H}^{\dagger} Q \bar{u}+F_{D} H^{\dagger} Q \bar{d}+F_{L} H^{\dagger} L \bar{e}+\text { h.c. } \\
& -\frac{M_{1}}{2} \widetilde{B} \widetilde{B}-\frac{M_{2}}{2} \widetilde{W^{I}} \widetilde{W^{I}}-\frac{M_{3}}{2} \widetilde{g}^{a} \widetilde{g}^{a}-\mu \widetilde{H}_{u}^{T} \epsilon \widetilde{H}_{d} \\
& -\frac{H^{\dagger}}{\sqrt{2}}\left(\kappa_{u} \sigma^{I} \widetilde{W^{I}}+\kappa_{u}^{\prime} \widetilde{B}\right) \widetilde{H}_{u}+\frac{\hat{H}^{\dagger}}{\sqrt{2}}\left(-\kappa_{d} \sigma^{I} \widetilde{W^{I}}+\kappa_{d}^{\prime} \widetilde{B}\right) \widetilde{H}_{d}+\text { h.c. }
\end{aligned}
$$

where $\hat{H}=-i \sigma_{2} H^{*}, H=\left(H^{+}, H^{0}\right)^{T}$, and $\epsilon=i \sigma_{2}$. The predictions for the Higgs mass will be derived from this Lagrangian using methods similar to the work of Sirlin and Zucchini on the SM Higgs boson [113] and the subsequent work of Hempfling and Kniehl on the SM top quark [114].

After electroweak symmetry breaking, the bare Higgs mass is related to the bare quartic coupling $\lambda_{0}$ and vacuum expectation value (vev) $v_{0}=\sqrt{2}\left\langle H^{0}\right\rangle$ by

$$
M_{h_{0}}(\bar{\mu})=\sqrt{\lambda_{0}(\bar{\mu})} v_{0}(\bar{\mu})
$$

where the dimensional regularization scale $\bar{\mu}$ is introduced into loop integrals through $\int d^{4} x \rightarrow \bar{\mu}^{-2 \epsilon} \int d^{d} x$ and is elevated to the renormalization scale in $\overline{M S}$. Each of these bare quantities must be related to physical quantities in order to obtain a meaningful relation. 
The pole mass is related to the bare mass by

$$
M_{h}^{2}=M_{h_{0}}^{2}(\bar{\mu})+\operatorname{Re} \Sigma_{h}\left(M_{h}, \bar{\mu}\right)+3 \frac{T_{h}(\bar{\mu})}{v},
$$

where $\Sigma_{h}$ is the Higgs self energy and $T_{h}$ is the tadpole ${ }^{1}$. The bare vev is related to the renormalized vev via muon decay [115] :

$$
v_{0}^{2}(\bar{\mu})=v_{F}^{2}\left(1+\Pi_{W W}(0, \bar{\mu})+E(\bar{\mu})-2 \frac{T_{h}(\bar{\mu})}{M_{H}^{2} v_{F}}\right)
$$

where $v_{F}=1 / \sqrt{\sqrt{2} G_{F}} \approx 246.22 \mathrm{GeV}, \Pi_{W W}(0, \bar{\mu})$ is the $W^{ \pm}$boson self-energy at zero momentum, and $E$ represents vertex and box corrections to muon decay in the standard model. Finally the bare coupling is related to the $\overline{M S}$ coupling by

$$
\lambda_{0}(\bar{\mu})=\lambda(\bar{\mu})+\frac{\beta_{\lambda}}{2} C_{U V},
$$

where $C_{U V}=\frac{1}{\epsilon}-\gamma_{E}+\log 4 \pi$ and $\beta_{\lambda}=\frac{\partial \lambda}{\partial \log \bar{\mu}}$. Putting these together, one finds

$$
\begin{aligned}
M_{h} & =\sqrt{\lambda(\bar{\mu})} v_{F}\left(1+\delta_{h}(\bar{\mu})\right) \\
\delta_{h}(\bar{\mu}) & =\frac{1}{2}\left(\frac{\operatorname{Re} \Sigma_{h}\left(M_{h}, \bar{\mu}\right)}{M_{h}^{2}}+\frac{T_{h}(\bar{\mu})}{M_{h}^{2} v_{F}}+E(\bar{\mu})+\Pi_{W W}(0, \bar{\mu})+\frac{\beta_{\lambda}}{2 \lambda} C_{U V}\right) .
\end{aligned}
$$

This formula includes all one-loop threshold and renormalization group ( $R G$ ) corrections, and can be improved to include the two-loop RG corrections to the running of $\lambda(\bar{\mu})$. The scale $\bar{\mu}$ should be chosen to minimize large logarithmic corrections, although at one-loop the $\bar{\mu}$ (and $C_{U V}$ ) dependence formally cancels from Eq.(7.6). The results for $\delta_{h}(\bar{\mu})$ in the SM were given in $[113]^{2}$. The split supersymmetry threshold corrections are discussed in detail in section 7.2.5.

\footnotetext{
${ }^{1}$ No tadpole counterterm is used in this chapter. It is a matter of convention whether or not one uses such a counterterm, and the final results are easily seen to be independent of this choice.

${ }^{2}$ The convention used here is related to $[113]$ by $\delta_{h}^{S M}($ this paper $)=-\frac{1}{2} \delta_{h}^{S M}([113])$
} 


\subsubsection{The algorithm used to calculate the Higgs mass}

The input parameters for the Higgs mass analysis include supersymmetry breaking scale $M_{S}, \tan \beta$ at $M_{S}$, and the soft gaugino and higgsino masses $M_{1}, M_{2}, M_{3}$, and $\mu$ (not to be confused with the RN scale $\bar{\mu}$ ) which are specified at the scale of gauge coupling unification $M_{G} \sim 3 \times 10^{16} \mathrm{GeV}$ and are assumed to be universal

$$
M_{1 / 2} \equiv M_{1}\left(M_{G}\right)=M_{2}\left(M_{G}\right)=M_{3}\left(M_{G}\right)=\mu\left(M_{G}\right)
$$

Of course, the $\mu$-term may take different values from the gaugino masses, but we have explicitly verified that the Higgs mass prediction is very insensitive to the $\mu$ initial value, so the results in Figs. $(7.1,7.2,7.3,7.4)$ are valid for most other reasonable values of $\mu$.

First, the coupled system of differential equations [58] for $g_{1}, g_{2}, g_{3}, F_{U}, F_{D}$, $F_{L}, \kappa_{u}, \kappa_{d}, \kappa_{u}^{\prime}, \kappa_{d}^{\prime}$ are solved numerically. The gauge couplings are run at two loops, whereas the seven other couplings are run at one loop. We keep only the top, bottom, and $\tau$ Yukawa couplings, and so we can replace $F_{U} \rightarrow Y_{t}, F_{D} \rightarrow Y_{b}, F_{L} \rightarrow Y_{\tau}$ in the all of the following formulae. The boundary values of the gauge couplings $g_{1}, g_{2}, g_{3}$ and Yukawa couplings $Y_{b}, Y_{\tau}$ are given at scale $M_{Z}$ from the latest world averages [116]. As will be discussed in section 7.2.3, $Y_{t}$ is given at the top pole mass $M_{t}$, including three-loop QCD corrections and one-loop threshold corrections from electro-weak and split supersymmetric interactions. The new split SUSY Yukawas are given at scale $M_{S}$ through the relations:

$$
\begin{aligned}
\kappa_{u}\left(M_{S}\right) & =g_{2}\left(M_{S}\right) \sin \beta \\
\kappa_{d}\left(M_{S}\right) & =g_{2}\left(M_{S}\right) \cos \beta \\
\kappa_{u}^{\prime}\left(M_{S}\right) & =\sqrt{\frac{3}{5}} g_{1}\left(M_{S}\right) \sin \beta \\
\kappa_{d}^{\prime}\left(M_{S}\right) & =\sqrt{\frac{3}{5}} g_{1}\left(M_{S}\right) \cos \beta .
\end{aligned}
$$

Because the boundary values for the couplings are given at different scales, it is necessary to take an iterative approach to solving the differential equations. The couplings 
which are specified at low scales, such as $y_{t}\left(M_{t}\right)$, are guessed at the high scale $M_{s}$, the differential equations are then solved, and the resulting value for $y_{t}\left(M_{t}\right)$ is compared to the correct value in order to obtain a better guess, at which point the procedure is repeated. Five iterations are usually sufficient. An additional complication arises because the split SUSY corrections to $Y_{t}\left(M_{t}\right)$ (detailed in section 7.2.3) depend on $U, V$, and $N$, which depend on the solutions of the RGE's for the gaugino/higgsino masses, which depend on the solutions of the RGE's for the dimensionless couplings, which in turn depend upon $Y_{t}\left(M_{t}\right)$. Thus, this entire analysis should be performed iteratively.

Armed with the RGE evolution of the dimensionless couplings, the RGE's for the soft masses $M_{1}, M_{2}, M_{3}$, and $\mu$ are then solved and are run down to scales $M_{1}, M_{2}$, $M_{3}$, and $\mu$, respectively, where the physical pole masses are extracted, as detailed in section 7.2.2. The chargino and neutralino mixing matrices $U, V$, and $N$ will appear in the threshold corrections.

In section 7.2.4 the two-loop running of the Higgs quartic coupling will be given. The solutions for $g_{1}, g_{2}, g_{3}, F_{U}, F_{D}, F_{L}, \kappa_{u}, \kappa_{d}, \kappa_{u}^{\prime}, \kappa_{d}^{\prime}$ yield the required inputs to solve the $\lambda$ RGE, with the gaugino and higgsino masses providing the appropriate matching scale between the Standard Model and split supersymmetric running. The boundary value of the Higgs quartic coupling in minimal split supersymmetry is

$$
\lambda\left(M_{S}\right)=\frac{1}{4}\left(g_{2}^{2}\left(M_{S}\right)+\frac{3}{5} g_{1}^{2}\left(M_{S}\right)\right) \cos ^{2} 2 \beta .
$$

Finally, all that remains is to include the finite threshold corrections in Eq.(7.6). These are detailed in section 7.2.5. Results for the Higgs mass are given in section 7.3 .

\subsubsection{The gaugino and higgsino mass spectrum}

The formulae for the running of the gaugino and higgsino masses are given in Eqs. (5763) of Ref.[58].

The gluino mass appears in our analysis as the threshold for the running of the 
strong coupling. It is straightforward to evaluate $M_{3}$ at scale $M_{3}$ and deduce the pole mass

$$
M_{\tilde{g}}=M_{3}\left(M_{3}\right)\left(1+12 \frac{g_{3}^{2}\left(M_{3}\right)}{(4 \pi)^{2}}\right) .
$$

The chargino and neutralino mass matrix diagonalization proceeds similar to the MSSM [5]. The mass matrices are given by

$$
X=\left(\begin{array}{cc}
M_{2} & \frac{\kappa_{u} v}{\sqrt{2}} \\
\frac{\kappa_{d} v}{\sqrt{2}} & \mu
\end{array}\right) \quad Y=\left(\begin{array}{cccc}
M_{1} & 0 & -\frac{\kappa_{d}^{\prime} v}{\sqrt{2}} & \frac{\kappa_{u}^{\prime} v}{\sqrt{2}} \\
0 & M_{2} & \frac{\kappa_{d} v}{\sqrt{2}} & -\frac{\kappa_{u} v}{\sqrt{2}} \\
-\frac{\kappa_{d}^{\prime} v}{\sqrt{2}} & \frac{\kappa_{d} v}{\sqrt{2}} & 0 & -\mu \\
\frac{\kappa_{u}^{u} v}{\sqrt{2}} & -\frac{\kappa_{u} v}{\sqrt{2}} & -\mu & 0
\end{array}\right)
$$

These are diagonalized by matrices $U, V$ in the chargino sector $\left(\chi_{i}^{+} \chi_{i}^{-}\right)$and $N$ in the neutralino sector $\left(\chi_{i}^{0}\right)$ :

$$
\chi_{i}^{+}=V_{i j} \psi_{j}^{+} \quad \chi_{i}^{-}=U_{i j} \psi_{j}^{-} \quad \chi_{i}^{0}=N_{i j} \psi_{j}^{0},
$$

where the gauge eigenstates are

$$
\psi_{j}^{+}=\left(\begin{array}{c}
\widetilde{W}^{+} \\
\widetilde{H}_{u}^{+}
\end{array}\right) \quad \psi_{j}^{-}=\left(\begin{array}{c}
\widetilde{W}^{-} \\
\widetilde{H}_{d}^{-}
\end{array}\right) \quad \psi_{j}^{0}=\left(\widetilde{B}, \widetilde{W}_{3}, \widetilde{H}_{d}^{0}, \widetilde{H}_{u}^{0}\right)^{T} .
$$

The matrices $U, V, N$ are specified by

$$
\begin{aligned}
N^{*} Y N^{-1}=M^{(N)} & =\operatorname{diag}\left\{M_{1}^{(N)}, M_{2}^{(N)}, M_{3}^{(N)}, M_{4}^{(N)}\right\} \\
N Y^{\dagger} Y N^{-1} & =\left(M^{(N)}\right)^{2} \\
U^{*} X V^{-1} & =M^{(C)}=\operatorname{diag}\left\{M_{1}^{(C)}, M_{2}^{(C)}\right\} \\
V X^{\dagger} X V^{-1} & =\left(M^{(C)}\right)^{2}=U^{*} X X^{\dagger} U^{T}
\end{aligned}
$$

The running mass parameters $M_{1}, M_{2}, \mu$ are evaluated at the scales $M_{1}, M_{2}, \mu$, respectively, in an iterative fashion. This minimizes the threshold corrections relating the pole masses and running masses, which are not considered in detail. In any case, the results are very insensitive to the exact scale chosen. The threshold effects due 
to gaugino masses are incorporated into the running of the dimensionless parameters appropriately.

\subsubsection{The Top Quark Yukawa coupling and pole mass}

Since the Higgs mass is most sensitive to the top Yukawa coupling, it is important to carefully extract this from the pole mass, which is taken from the 2005 summer average of $\mathrm{CDF}$ and D0 [117] to be $M_{t}=172.7 \pm 2.9 \mathrm{GeV}$. The leading corrections are from QCD and were calculated to two-loops in [118] and to three-loops in [119]. The full electro-weak corrections at one-loop were considered in [114], where the authors found the following relation between the pole mass, the $\overline{M S}$ Yukawa coupling, and the vacuum-expectation-value $v_{F}$ :

$$
y_{t}(\bar{\mu})=\sqrt{2} \frac{M_{t}}{v_{F}}\left(1+\delta_{t}(\bar{\mu})\right) .
$$

The correction term is derived analogous to Eq.(7.6) and is given by

$$
\delta_{t}(\bar{\mu})=\operatorname{Re} \Sigma_{t}\left(M_{t}, \bar{\mu}\right)-\frac{\Pi_{W W}(0, \bar{\mu})}{2 M_{W}^{2}}-\frac{E(\bar{\mu})}{2}-\frac{\beta_{y_{t}}}{2 y_{t}} C_{U V} .
$$

In this formula $\Sigma_{t}$ represents the top quark self energy and $E$ is the vertex and box corrections to muon decay, neither of which receive new contributions in split SUSY at one-loop. However, the $W$ boson self energy, $\Pi_{W W}$, does receive corrections, which are calculated below. The UV divergence, $C_{U V}$, multiplying the top Yukawa beta function $\beta_{y_{t}}=\bar{\mu} \frac{\partial y_{t}}{\partial \bar{\mu}}$.comes from the relation between the bare and $\overline{M S}$ Yukawa coupling and is canceled by the divergent parts of $\Sigma_{t}, \Pi_{W W}$, and $E$.

It is convenient to decompose the correction term into parts arising from QCD, electro-weak theory (EW), and split-supersymmetry (SS) :

$$
\delta_{t}(\bar{\mu})=\delta_{t}^{Q C D}(\bar{\mu})+\delta_{t}^{E W}(\bar{\mu})+\delta_{t}^{S S}(\bar{\mu})
$$

The three-loop QCD term derived from [118][119] for the top quark at scale $\bar{\mu}=M_{t}$ 
is

$$
\begin{aligned}
\delta_{t}^{Q C D}\left(\bar{\mu}=M_{t}\right) & \approx-\frac{4}{3}\left(\frac{\alpha_{3}\left(M_{t}\right)}{\pi}\right)-9.1\left(\frac{\alpha_{3}\left(M_{t}\right)}{\pi}\right)^{2}-80\left(\frac{\alpha_{3}\left(M_{t}\right)}{\pi}\right)^{3} \\
& \approx-0.046-0.011-0.003 \approx-0.060
\end{aligned}
$$

for $\alpha_{3}\left(M_{Z}\right)=0.118$. While the EW term given in Ref.[114] formally depends on $M_{h}$ and $M_{t}$, it turns out that in the range of Higgs and top masses of interest, this contribution is negligible $\left|\delta_{t}^{E W}\right|<0.001$.

Now we turn to the SS corrections, which arise only from the gaugino and higgsino contribution to the $W^{ \pm}$self-energy. The $W^{ \pm}-\chi_{i}^{0}-\chi_{j}^{ \pm}$vertex is given by $i g \gamma_{\mu}\left(L_{i j} P_{L}+\right.$ $\left.R_{i j} P_{R}\right)$, with

$$
\begin{aligned}
L_{i j} & =-\frac{1}{\sqrt{2}} N_{i 4} V_{j 2}^{*}+N_{i 2} V_{j 1}^{*} \\
R_{i j} & =\frac{1}{\sqrt{2}} N_{i 3}^{*} U_{j 2}+N_{i 2}^{*} U_{j 1} .
\end{aligned}
$$

This vertex is used to derive the split SUSY corrections involving charginos and neutralinos :

$$
\begin{aligned}
16 & \pi^{2} \Pi_{W W}^{(C, N)}(0, \bar{\mu})=-2 M_{W}^{2} C_{U V} X_{2}(S S)+g^{2} \sum_{i=1}^{4} \sum_{j=1}^{2}\left(\left(L_{i j} L_{i j}^{*}+R_{i j} R_{i j}^{*}\right)\right. \\
\times & {\left[a^{2}\left(\log \frac{a^{2}}{\bar{\mu}^{2}}-1 / 2\right)+b^{2}\left(\log \frac{b^{2}}{\bar{\mu}^{2}}-1 / 2\right)+\frac{a^{2} b^{2}}{a^{2}-b^{2}} \log \frac{a^{2}}{b^{2}}\right] } \\
& \left.+2\left(L_{i j} R_{i j}^{*}+R_{i j} L_{i j}^{*}\right) \frac{a b}{a^{2}-b^{2}}\left[-a^{2}\left(\log \frac{a^{2}}{\bar{\mu}^{2}}-1\right)+b^{2}\left(\log \frac{b^{2}}{\bar{\mu}^{2}}-1\right)\right]\right),
\end{aligned}
$$

where we used the shorthand $a=M_{j}^{(C)}, b=M_{i}^{(N)}$ and $X_{2}(S S)$ is given in Eq.(7.23). The resulting correction term

$$
\delta_{t}^{S S}(\bar{\mu})=-\left.\frac{\Pi_{W W}^{(C, N)}(0, \bar{\mu})}{2 M_{W}^{2}}\right|_{C_{U V}=0}
$$

depends on the soft gaugino mass terms, $\tan \beta$, and the scalar mass scale $M_{S}$. The 
scale $\bar{\mu}$ must be chosen in accordance with the decoupling scale $M_{S S}$ imposed on $y_{t}$ at the chargino/neutralino thresholds, $\bar{\mu}=M_{S S}$. The exact scale is not very important, but consistently applying the choice to both the running of $y_{t}$ and the threshold correction $\delta_{t}^{S S}$ is important. For the explicit results given in Figs. $(7.1,7.2,7.3,7.4)$, the decoupling scale was chosen to be the mass of the lightest supersymmetric particle, which is typically a neutralino. Generically the split SUSY correction is small, $\left|\delta_{t}^{S S}\left(\bar{\mu}=M_{S S}\right)\right| \lesssim 0.01$, but should be included since it can lead to a shift in the Higgs mass of up to $2 \mathrm{GeV}$.

To summarize, we have found

$$
y_{t}\left(M_{t}\right)=0.945\left(\frac{M_{t}}{175 \mathrm{GeV}}\right)\left(1+\delta_{t}^{S S}\left(M_{S S}\right)\right)
$$

\subsubsection{The 2-loop running of the Higgs Quartic Coupling}

It is useful to define the following invariants involving the standard model Yukawa couplings and the new split SUSY Yukawa couplings :

$$
\begin{aligned}
Y_{2}(S M) & =\operatorname{Tr}\left[3 F_{U}^{\dagger} F_{U}+3 F_{D}^{\dagger} F_{D}+F_{L}^{\dagger} F_{L}\right] \\
Y_{4}(S M) & =\operatorname{Tr}\left[3\left(F_{U}^{\dagger} F_{U}\right)^{2}+3\left(F_{D}^{\dagger} F_{D}\right)^{2}+\left(F_{L}^{\dagger} F_{L}\right)^{2}\right] \\
Y_{6}(S M) & =\operatorname{Tr}\left[3\left(F_{U}^{\dagger} F_{U}\right)^{3}+3\left(F_{D}^{\dagger} F_{D}\right)^{3}+\left(F_{L}^{\dagger} F_{L}\right)^{3}\right] \\
Y_{G}(S M) & =\left(\frac{17}{20} g_{1}^{2}+\frac{9}{4} g_{2}^{2}+8 g_{3}^{2}\right) \operatorname{Tr}\left(F_{U}^{\dagger} F_{U}\right) \\
& +\left(\frac{1}{4} g_{1}^{2}+\frac{9}{4} g_{2}^{2}+8 g_{3}^{2}\right) \operatorname{Tr}\left(F_{D}^{\dagger} F_{D}\right)+\frac{3}{4}\left(g_{1}^{2}+g_{2}^{2}\right) \operatorname{Tr}\left(F_{L}^{\dagger} F_{L}\right) \\
X_{2}(S S) & =3\left(\kappa_{u}^{2}+\kappa_{d}^{2}\right)+\kappa_{u}^{\prime 2}+\kappa_{d}^{\prime 2} \\
X_{4}(S S) & =5\left(\kappa_{u}^{4}+\kappa_{d}^{4}\right)+2 \kappa_{u}^{2} \kappa_{d}^{2}+2\left(\kappa_{u} \kappa_{u}^{\prime}+\kappa_{d} \kappa_{d}^{\prime}\right)^{2}+\left(\kappa_{u}^{\prime 2}+\kappa_{d}^{\prime 2}\right)^{2} .
\end{aligned}
$$

The running of the quartic coupling $\lambda$ of the Higgs boson is governed by

$$
\beta_{\lambda} \equiv \bar{\mu} \frac{\partial \lambda}{\partial \bar{\mu}}=\frac{1}{16 \pi^{2}} \beta_{\lambda}^{(1)}+\frac{1}{\left(16 \pi^{2}\right)^{2}} \beta_{\lambda}^{(2)}+\cdots
$$


The one-loop beta function is given by [58]

$$
\begin{aligned}
\beta_{\lambda}^{(1)} & =12 \lambda^{2}-9 \lambda\left(\frac{1}{5} g_{1}^{2}+g_{2}^{2}\right)+\left(\frac{27}{100} g_{1}^{4}+\frac{9}{10} g_{1}^{2} g_{2}^{2}+\frac{9}{4} g_{2}^{4}\right) \\
& +4 \lambda Y_{2}(S M)-4 Y_{4}(S M)+2 \lambda X_{2}(S S)-X_{4}(S S)
\end{aligned}
$$

The 2 loop result is conveniently divided into two terms,

$$
\beta_{\lambda}^{(2)}=\beta_{\lambda}^{(2)}\left(S M^{\prime}\right)+\beta_{\lambda}^{(2)}(S S)
$$

where $S S$ is the new split SUSY contribution and $S M^{\prime}$ denotes the standard model result modified to include gauginos and higgsinos in gauge boson self-energies. This is accomplished by replacing the number of generations in the SM result with

$$
\begin{gathered}
N_{g}(1)=3+3 / 10=33 / 10 \quad N_{g}(2)=3+3 / 2=9 / 2 \quad(7.27) \\
\beta_{\lambda}^{(2)}\left(S M^{\prime}\right)=-78 \lambda^{3}-24 \lambda^{2} Y_{2}(S M)-\lambda Y_{4}(S M)-42 \lambda \operatorname{Tr}\left(F_{U}^{\dagger} F_{U} F_{D}^{\dagger} F_{D}\right)+20 Y_{6}(S M) \\
-\quad 12 \operatorname{Tr}\left[F_{U}^{\dagger} F_{U}\left(F_{U}^{\dagger} F_{U}+F_{D}^{\dagger} F_{D}\right) F_{D}^{\dagger} F_{D}\right]+10 \lambda Y_{G}(S M)+54 \lambda^{2}\left(g_{2}^{2}+\frac{1}{5} g_{1}^{2}\right) \\
-\quad \lambda\left[\left(\frac{313}{8}-10 N_{g}(2)\right) g_{2}^{4}-\left(\frac{687}{200}+2 N_{g}(1)\right) g_{1}^{4}-\frac{117}{20} g_{2}^{2} g_{1}^{2}\right] \\
-\quad 64 g_{3}^{2} \operatorname{Tr}\left[\left(F_{U}^{\dagger} F_{U}\right)^{2}+\left(F_{D}^{\dagger} F_{D}\right)^{2}\right]-\frac{8}{5} g_{1}^{2} \operatorname{Tr}\left[2\left(F_{U}^{\dagger} F_{U}\right)^{2}-\left(F_{D}^{\dagger} F_{D}\right)^{2}+3\left(F_{L}^{\dagger} F_{L}\right)^{2}\right] \\
-\quad \frac{3}{2} g_{2}^{4} Y_{2}(S M)+g_{1}^{2}\left[\left(\frac{63}{5} g_{2}^{2}-\frac{171}{50} g_{1}^{2}\right) \operatorname{Tr}\left(F_{U}^{\dagger} F_{U}\right)+\left(\frac{27}{5} g_{2}^{2}+\frac{9}{10} g_{1}^{2}\right) \operatorname{Tr}\left(F_{D}^{\dagger} F_{D}\right)\right. \\
\left.+\left(\frac{33}{5} g_{2}^{2}-\frac{9}{2} g_{1}^{2}\right) \operatorname{Tr}\left(F_{L}^{\dagger} F_{L}\right)\right]+\left(\frac{497}{8}-8 N_{g}(2)\right) g_{2}^{6}-\left(\frac{97}{40}+\frac{8}{5} N_{g}(2)\right) g_{2}^{4} g_{1}^{2} \\
-\left(\frac{717}{200}+\frac{8}{5} N_{g}(1)\right) g_{2}^{2} g_{1}^{4}-\left(\frac{531}{1000}+\frac{24}{25} N_{g}(1)\right) g_{1}^{6}
\end{gathered}
$$


The new split SUSY Yukawas contribute

$$
\begin{aligned}
\beta_{\lambda}^{(2)}(S S) & =-12 \lambda^{2} X_{2}(S S)-\frac{\lambda}{4}\left[5\left(\kappa_{u}^{4}+\kappa_{d}^{4}\right)+44 \kappa_{u}^{2} \kappa_{d}^{2}+2\left(\kappa_{u}^{2} \kappa_{u}^{\prime 2}+\kappa_{d}^{2} \kappa_{d}^{\prime 2}\right)+\kappa_{u}^{\prime 4}+\kappa_{d}^{4}\right. \\
& \left.-12 \kappa_{u}^{\prime 2} \kappa_{d}^{\prime 2}-80 \kappa_{u} \kappa_{d} \kappa_{u}^{\prime} \kappa_{d}^{\prime}\right]+\frac{47}{2}\left(\kappa_{u}^{6}+\kappa_{d}^{6}\right)+\frac{5}{2}\left(\kappa_{u}^{\prime 6}+\kappa_{d}^{\prime 6}\right)+\frac{7}{2} \kappa_{u}^{2} \kappa_{d}^{2}\left(\kappa_{u}^{2}+\kappa_{d}^{2}\right) \\
& +\frac{11}{2}\left(\kappa_{u}^{4} \kappa_{u}^{\prime 2}+\kappa_{d}^{4} \kappa_{d}^{\prime 2}\right)+\frac{21}{2} \kappa_{u}^{2} \kappa_{d}^{2}\left(\kappa_{u}^{\prime 2}+\kappa_{d}^{\prime 2}\right)+19 \kappa_{u} \kappa_{d} \kappa_{u}^{\prime} \kappa_{d}^{\prime}\left(\kappa_{u}^{2}+\kappa_{d}^{2}\right) \\
& +21 \kappa_{u} \kappa_{d} \kappa_{u}^{\prime} \kappa_{d}^{\prime}\left(\kappa_{u}^{\prime 2}+\kappa_{d}^{\prime 2}\right)+\frac{17}{2}\left(\kappa_{u}^{2} \kappa_{u}^{\prime 4}+\kappa_{d}^{2} \kappa_{d}^{\prime 4}+\kappa_{u}^{\prime 2} \kappa_{d}^{\prime 4}+\kappa_{u}^{\prime 4} \kappa_{d}^{\prime 2}\right) \\
& +\frac{19}{2} \kappa_{u}^{\prime 2} \kappa_{d}^{\prime 2}\left(\kappa_{u}^{2}+\kappa_{d}^{2}\right)+\frac{15}{4} \lambda\left[\left(g_{2}^{2}+\frac{1}{5} g_{1}^{2}\right) X_{2}(S S)+8 g_{2}^{2}\left(\kappa_{u}^{2}+\kappa_{d}^{2}\right)\right] \\
& -4 g_{2}^{2}\left[5\left(\kappa_{u}^{4}+\kappa_{d}^{4}\right)+2 \kappa_{u}^{2} \kappa_{d}^{2}+\left(\kappa_{u} \kappa_{u}^{\prime}+\kappa_{d} \kappa_{d}^{\prime}\right)^{2}\right]-g_{2}^{4}\left[\frac{3}{4} X_{2}(S S)+36\left(\kappa_{u}^{2}+\kappa_{d}^{2}\right)\right] \\
& +\frac{3}{10} g_{1}^{2} g_{2}^{2}\left[21\left(\kappa_{u}^{2}+\kappa_{d}^{2}\right)-\left(\kappa_{u}^{\prime 2}+\kappa_{d}^{\prime 2}\right)\right]-\frac{9}{100} g_{1}^{4} X_{2}(S S) .
\end{aligned}
$$

In deriving these, I relied on the useful papers of Luo, Wang, and Xiao [47], which corrected some typographical errors from the seminal works of Machacek and Vaughn [46]. Another useful paper is Ref.[120].

\subsubsection{The Higgs Self Energy and Tadpole Corrections}

In split supersymmetry there are corrections to $\Sigma_{h}, T_{h}$, and $\Pi_{W W}$, but not to $E$.

The Higgs tadpole and self-energy depend on the mass mixing matrices which appear in the Feynman rules. The interaction Lagrangian in terms of the physical mass eigenstate Dirac and Majorana fermions is given by

$$
\mathcal{L}_{i n t}=-\frac{h}{\sqrt{2}} \bar{\psi}_{i}^{ \pm}\left(P_{L} L_{i j}^{C}+P_{R} R_{i j}^{C}\right) \psi_{j}^{ \pm}+\frac{h}{2} \bar{\psi}_{i}^{0}\left(P_{L}\left(R_{(i j)}^{N}\right)^{*}+P_{R} R_{(i j)}^{N}\right) \psi_{j}^{0}
$$

where the mixing matrices are

$$
\begin{aligned}
R_{i j}^{C} & =\left(L_{j i}^{C}\right)^{*}=\kappa_{u} V_{i 2} U_{j 1}+\kappa_{d} V_{i 1} U_{j 2} \\
R_{i j}^{N} & =\left(\kappa_{u} N_{i 2}-\kappa_{u}^{\prime} N_{i 1}\right) N_{j 4}-\left(\kappa_{d} N_{i 2}-\kappa_{d}^{\prime} N_{i 1}\right) N_{j 3} \\
R_{(i j)}^{N} & =\frac{1}{2}\left(R_{i j}^{N}+R_{j i}^{N}\right) .
\end{aligned}
$$


The split-supersymmetric contribution to the Higgs tadpole $i T_{h}=i\left(T_{h}^{(C)}+T_{h}^{(N)}\right)$ involves charginos and neutralinos:

$$
\begin{gathered}
16 \pi^{2} T_{h}^{(C)}(\bar{\mu})=-2 \sqrt{2} \sum_{i=1}^{2} \operatorname{Re}\left[R_{i i}^{C}\left(M_{i}^{C}\right)^{3}\left(C_{U V}-\log \frac{\left(M_{i}^{C}\right)^{2}}{\bar{\mu}^{2}}+1\right)\right] \\
16 \pi^{2} T_{h}^{(N)}(\bar{\mu})=2 \sum_{i=1}^{4} \operatorname{Re}\left[R_{(i i)}^{N}\left(M_{i}^{N}\right)^{3}\left(C_{U V}-\log \frac{\left(M_{i}^{C}\right)^{2}}{\bar{\mu}^{2}}+1\right)\right]
\end{gathered}
$$

The Higgs self energies are easily written in terms of the canonical one-loop basis functions $A(M), B_{0}\left(k^{2} ; M_{1}, M_{2}\right)[121]$ :

$$
\begin{aligned}
16 & \pi^{2} \Sigma_{h}^{(C)}\left(p^{2}, \bar{\mu}\right)=\sum_{i, j=1}^{2}\left[2 \operatorname{Re} M_{i} M_{j} R_{i j}^{C}\left(L_{i j}^{C}\right)^{*} B_{0}\left(p^{2} ; M_{i}, M_{j}\right)\right. \\
& \left.+\frac{\left(\left|L_{i j}^{C}\right|^{2}+\left|R_{i j}^{C}\right|^{2}\right)}{2}\left(A\left(M_{i}\right)+A\left(M_{j}\right)+\left(M_{i}^{2}+M_{j}^{2}-p^{2}\right) B_{0}\left(p^{2} ; M_{i}, M_{j}\right)\right)\right] \\
16 & \pi^{2} \Sigma_{h}^{(N)}\left(p^{2}, \bar{\mu}\right)=\sum_{i, j=1}^{4}\left[2 \operatorname{Re} M_{i} M_{j} R_{(i j)}^{N}\left(R_{(i j)}^{N}\right)^{*} B_{0}\left(p^{2} ; M_{i}, M_{j}\right)\right. \\
& \left.+\left|R_{(i j)}^{N}\right|^{2}\left(A\left(M_{i}\right)+A\left(M_{j}\right)+\left(M_{i}^{2}+M_{j}^{2}-p^{2}\right) B_{0}\left(p^{2} ; M_{i}, M_{j}\right)\right)\right]
\end{aligned}
$$

The above results lead to the following correction term for the Higgs mass:

$\delta_{h}^{S S}(\bar{\mu})=\left.\frac{1}{2}\left(\frac{\Sigma_{h}^{(C)}\left(M_{h}, \bar{\mu}\right)+\Sigma_{h}^{(N)}\left(M_{h}, \bar{\mu}\right)}{M_{h}^{2}}+\frac{T_{h}^{(C)}(\bar{\mu})+T_{h}^{(N)}(\bar{\mu})}{M_{h}^{2} v_{F}}+\Pi_{W W}^{(C N)}(0, \bar{\mu})\right)\right|_{C_{U V}=0}$.

As discussed below Eq. (7.21), the scale $\bar{\mu}$ must be chosen in accordance with the decoupling imposed on $\lambda$ at the chargino/neutralino thresholds, $\bar{\mu}=M_{S S}$. Combined with the SM results of [113], which should be evaluated at $M_{h}$, the total threshold correction is given by

$$
\begin{aligned}
M_{h} & =\sqrt{\lambda\left(M_{h}\right)} v_{F}\left(1+\delta_{h}\left(M_{h}\right)\right) \\
\delta_{h}\left(M_{h}\right) & =\delta_{h}^{S M}\left(M_{h}\right)+\delta_{h}^{S S}\left(M_{S S}\right) .
\end{aligned}
$$


A useful check of these results is the cancelation of divergences $C_{U V}$ in Eq.(7.6). This involves repeated use of the definitions in Eq.(7.14), and has been explicitly verified.

\subsection{Results for the Higgs Mass}

The corrections to the Higgs mass considered in this chapter are of three varieties:

- Top Yukawa Coupling. The threshold corrections to the Yukawa coupling initial value given in Eq.(7.17) are amplified because $y_{t}$ is raised to the fourth power in $\beta_{\lambda}^{(1)}$. The QCD corrections to $y_{t}\left(M_{t}\right)$ are dominant $(\sim-6 \%)$ and lead to a downward shift in the Higgs mass of about $15 \mathrm{GeV}$. The electro-weak corrections are negligible over the entire parameter range of interest.

The split SUSY correction is small but not negligible. For each choice of parameters $\tan \beta, M_{s}$, and $M_{1 / 2}$, there will be a correction term $\delta_{t}^{S S}$ to the initial value $y_{t}\left(M_{t}\right)$. However, $y_{t}\left(M_{t}\right)$ is required input for solving the coupled differential equations which eventually lead to $\delta_{t}^{S S}$. Thus, in principle, an iterative approach must be taken. After performing this type of analysis we found that it could be circumvented by using the output $\delta_{t}^{S S}$ along with the following simple rule of thumb: every shift in $y_{t}\left(M_{t}\right)$ of \pm 0.0045 will shift the Higgs mass by $\pm 1 \mathrm{GeV}$. The contributions of the bottom and $\tau$ Yukawa couplings turn out to be completely negligible and can be omitted from the beginning.

- Two-loop running of $\lambda$. The two-loop correction to the beta function is numerically very small, which is partially due to cancelations between the SM and split SUSY contributions in Eqs. $(7.28,7.29)$. The shift in the Higgs mass due to including $\beta_{\lambda}^{(2)}$ is less than $300 \mathrm{MeV}$ for all relevant values of $M_{s}, \tan \beta$, and $M_{1 / 2}$.

- Threshold corrections $\left(\delta_{h}\right)$. The correction given in Eq.(7.36) typically pushes down the Higgs mass by several $\mathrm{GeV}$, with a larger shift occurring for small $\tan \beta$ and small $M_{s}$. Typically, the SM contributes most of this shift, with the split SUSY corrections $\lesssim 1 \mathrm{GeV}$. 
All of the above corrections should be considered in the context of two sources of uncertainty. First, the uncertainties in the top mass $M_{t}=172.7 \pm 2.9 \mathrm{GeV}$ and $\alpha_{s}\left(M_{z}\right)=0.118 \pm 0.003$ translate into uncertainties in $M_{h}$ of about $\pm(3-5) \mathrm{GeV}$ and $\mp(0.3-1.2) \mathrm{GeV}$, respectively. Second, there are model specific "theory uncertainties" at the high scale [104].

Some representative plots of the Higgs mass are shown in Figs. $(7.1,7.2)$. In Figs. $(7.3,7.4)$, the two-loop and threshold corrections discussed above are plotted. The large QCD corrections to $M_{h}(\sim-15 \mathrm{GeV})$ arising from Eq.(7.18) are not shown explicitly in Figs. $(7.3,7.4)$ in order to clearly illustrate the other much smaller effects. 


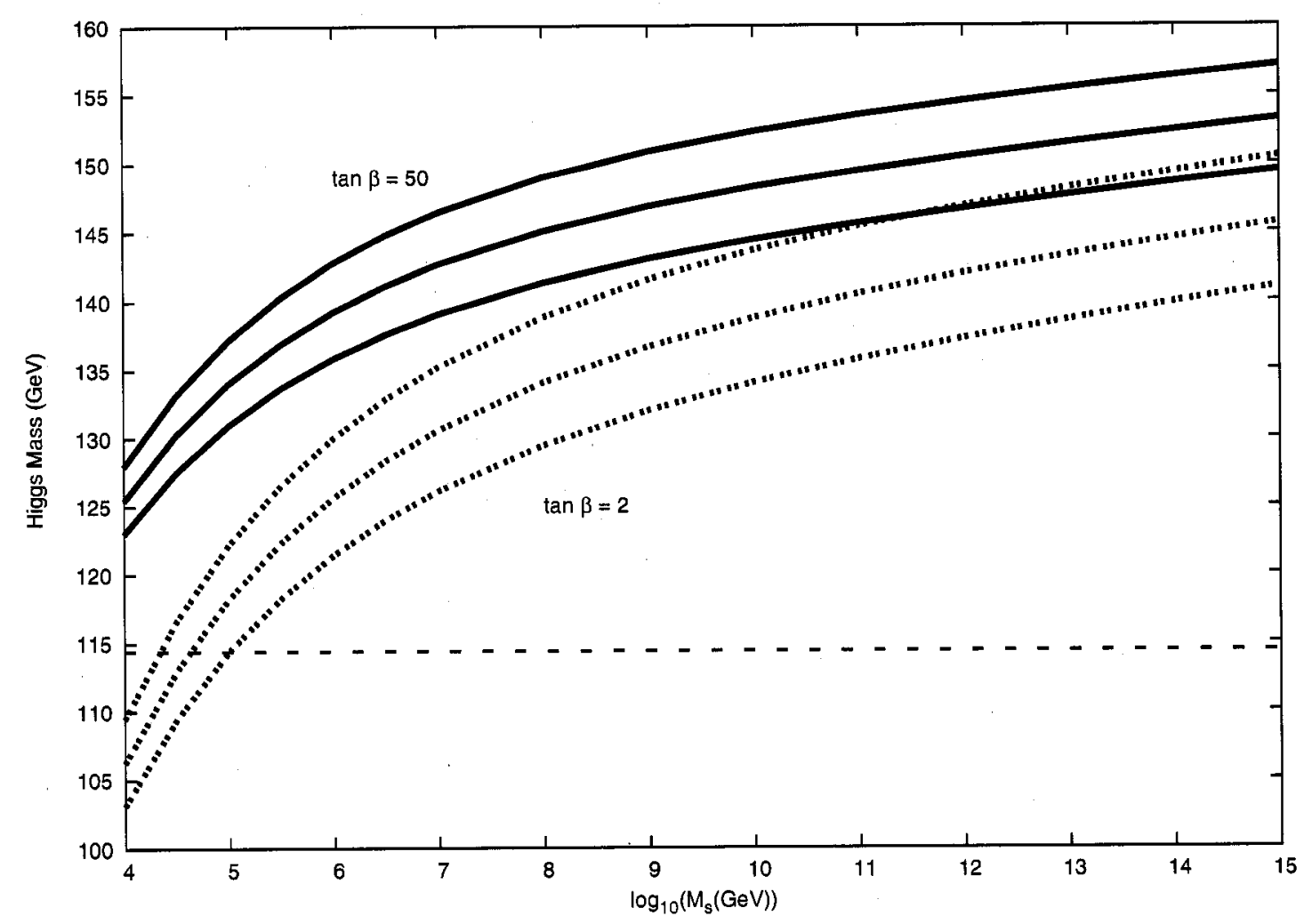

Figure 7.1: The Higgs mass prediction versus $M_{s}$ for $\tan \beta=2$ (lower dotted lines) and $\tan \beta=50$ (upper solid lines). For each set of three the middle line is with $\alpha_{s}\left(M_{z}\right)=$ $0.118, M_{t}=172.7 \mathrm{GeV}$; the upper line is with $\alpha_{s}\left(M_{z}\right)=0.115, M_{t}=175.6 \mathrm{GeV}$; and the lower line is with $\alpha_{s}\left(M_{z}\right)=0.121, M_{t}=169.8 \mathrm{GeV}$. These correspond to the $1 \sigma$ variations of $\alpha_{s}\left(M_{Z}\right)=0.118 \pm 0.003$ and $M_{t}=172.7 \pm 2.9 \mathrm{GeV}$, with the resulting uncertainties in the Higgs mass considered additively. The gaugino and higgsino masses at $M_{G}$ are taken as universal $M_{1 / 2}=500 \mathrm{GeV}$. The experimental lower bound [122] of $M_{h}>114.4 \mathrm{GeV}$ (at 95\%) is shown. 


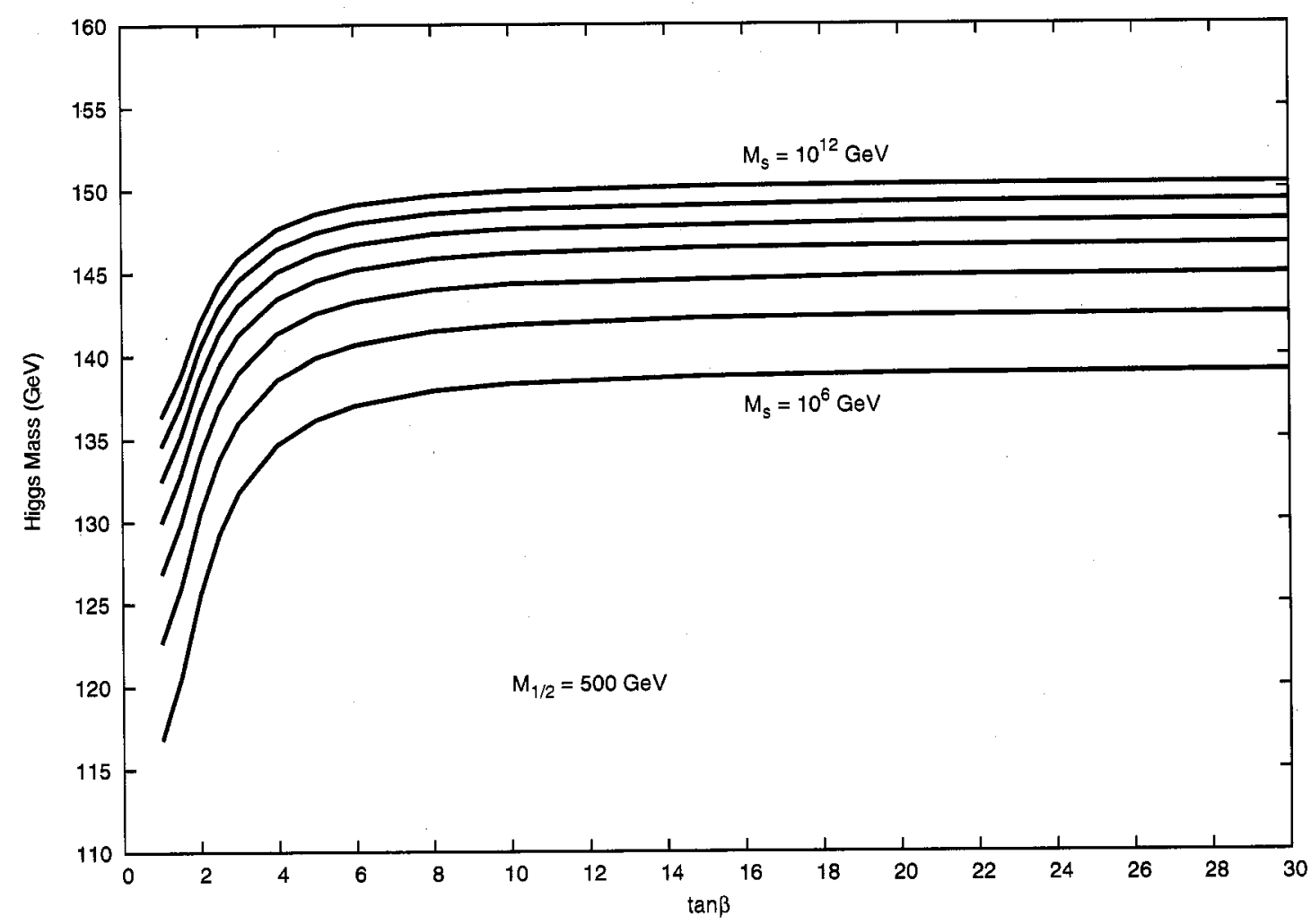

Figure 7.2: The Higgs mass versus $\tan \beta$, for $M_{s}=10^{6}, 10^{7}, 10^{8}, 10^{9}, 10^{10}, 10^{11}$, and $10^{12} \mathrm{GeV}$, from bottom to top. Here $\alpha_{s}\left(M_{z}\right)=0.118$ and $M_{t}=172.7 \mathrm{GeV}$. 


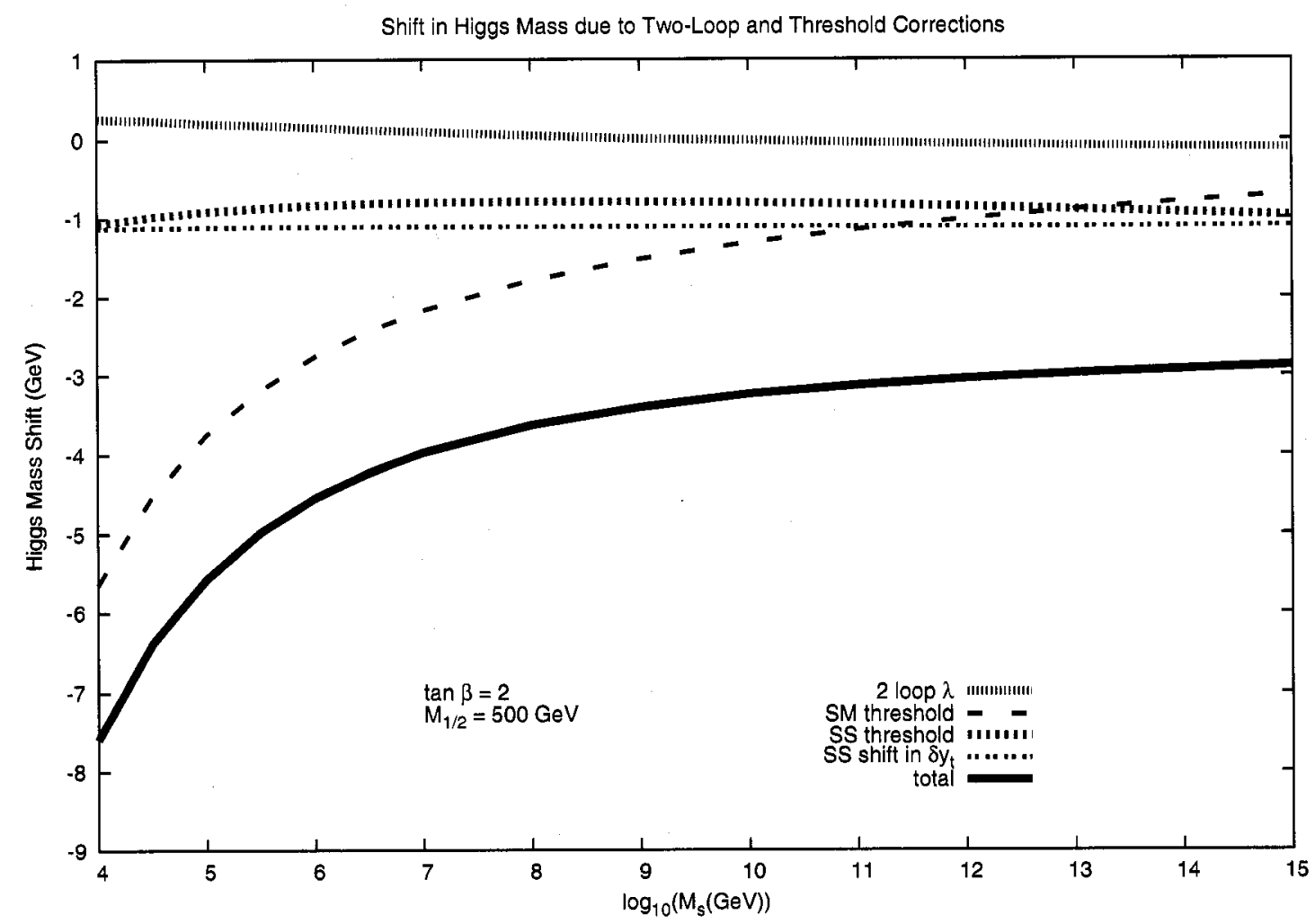

Figure 7.3: The Higgs mass shift for $\tan \beta=2$ and $M_{1 / 2}=500 \mathrm{GeV}$ due to four types of threshold corrections. The correction due to the two-loop running of $\lambda$ is shown in the thick dense dotted line near zero. The Standard Model (SM) correction from [113] is the dashed line. The split SUSY (SS) correction from Eq.(7.35) is the thick dotted line. The thin dotted line is the SS correction to the Higgs mass through the correction to the top Yukawa initial value, Eq.(7.21). The solid line is the total of these four corrections. Here $\alpha_{s}\left(M_{z}\right)=0.118$ and $M_{t}=172.7 \mathrm{GeV}$. 


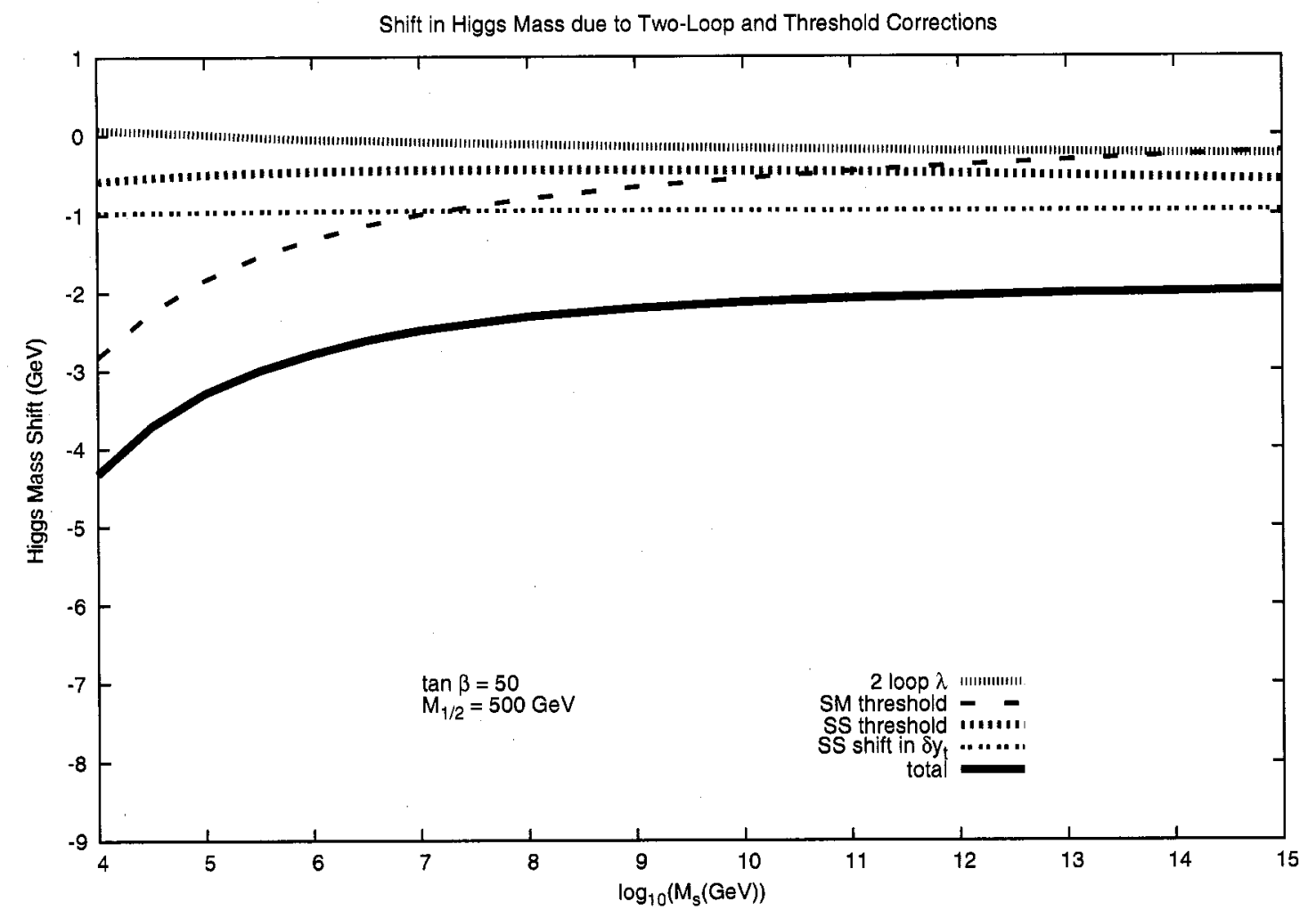

Figure 7.4: Same as Fig.7.3 except with $\tan \beta=50$. 


\section{Chapter 8}

\section{On the Structure of Perturbation Theory}

\subsection{Predicting Higher Order Terms Using BLM methods}

Even without considering the complicated diagrammatic construction of a QCD effective charge analogous to QED, the BLM method can be used to reliably estimate the renormalization scale. This is done by choosing the scale at each order to absorb the terms arising from the running of the coupling, which are identified by the dependence on the number of flavors $f$. In this approach, the renormalization scale ambiguity problem is closely related to the conformal expansion, since after BLM scale fixing, the series takes exactly the same form as a related conformal theory. In this context, the 'conformal' terms are those that would arise in a theory with zero $\beta$ function, i.e. without any breaking of scale invariance. Likewise, the non-conformal terms are those which are associated with the running of the coupling.

One way to test the efficacy of BLM scale-fixing methods is by verifying that in fact the BLM scales do "automatically re-sum" the dominant part of higher order corrections. In particular, starting with some series truncated at order $N$, we can use BLM methods to predict part of the order $N+1$ term, and then compare with known 
results.

In this section, a prediction for the NNNLO $\left(\mathcal{O}\left(\alpha_{s}^{4}\right)\right)$ term in the hadronic decay rate of Higgs bosons will be estimated, and compared with the exact calculation. The explicit results are derived in [123][124] by calculating the Higgs two-point function with a quark loop dressed by QCD corrections. The optical theorem then relates the imaginary part to the partial decay rates.

The partial decay width of the Higgs boson into a quark-antiquark pair is

$$
\begin{aligned}
\Gamma(H \rightarrow f \bar{f}) & =\frac{G_{F} M_{H}}{4 \sqrt{2} \pi} m_{f}^{2} \widetilde{R}\left(s=M_{H}^{2}\right) \\
\widetilde{R}(s) & =1+\frac{17}{3} a_{H}(s),
\end{aligned}
$$

where the effective charge $a_{H}(s)$ is defined in the second line. The perturbative expansion is given by

$$
a_{H}(s)=\bar{a}(s)+\bar{a}^{2}(s)(A+B f)+\bar{a}^{3}(s)\left(C+D f+E f^{2}\right)+\cdots
$$

where $\bar{a}(s)=\alpha_{\overline{M S}}(s) / \pi$. The values for the Higgs scalar two-point correlator are

$$
A=6.35 \quad B=-0.24 \quad C=29.0 \quad D=-4.55 \quad E=0.0458
$$

Using Eqns.(3.6-3.16) of [125] we can rewrite the result as a conformal relation

$$
a_{H}(s)=\bar{a}\left(Q_{1}^{2}\right)+\widetilde{A} \bar{a}^{2}\left(Q_{2}^{2}\right)+\widetilde{C} \bar{a}^{3}\left(Q_{2}^{2}\right),
$$

where

$$
\begin{aligned}
\widetilde{A} & =A+\frac{11 C_{A}}{2} B \approx 2.39 \\
\widetilde{C} & =-\frac{3 C_{A}}{8}\left(7 C_{A}+11 C_{F}\right) B+C+\frac{11 C_{A}}{2} D+\frac{121 C_{A}^{2}}{4} E \approx-24.0 \\
Q_{1} & =\sqrt{s} \exp \left(3 B+18\left(B^{2}-E\right) \beta_{0} \bar{a}\right) \approx 0.49 \sqrt{s} \\
Q_{2} & =\sqrt{s} \exp \left(\frac{-57 B+12 D+396 E}{8 \widetilde{A}}\right) \approx 0.30 \sqrt{s}
\end{aligned}
$$


The scales $Q_{1}$ and $Q_{2}$ are the BLM scales for the LO and NLO terms. The scales are chosen so that all of the non-conformal terms are summed into the running coupling. These are approximations to the physical virtualities of the gluons and control the effective number of flavors in the running coupling. The terms $\widetilde{A}, \widetilde{C}$ are known as the conformal coefficients at NLO and NNLO, respectively.

We now will try to predict the NNNLO terms associated with the running of the QCD coupling. In general one might expect such terms to have renormalon $n$ ! growth.

The $\beta$ function for $\overline{M S}$ through 3 loops is given by

$$
\begin{aligned}
& \bar{\beta}=\frac{\partial \bar{a}}{\partial \log q^{2}}=-\bar{a}^{2}\left[\beta_{0}+\beta_{1} \bar{a}+\bar{\beta}_{2} \bar{a}^{2}+\cdots\right] \\
& \left.\beta_{0}=(11-2 f / 3)\right) / 4 \\
& \beta_{1}=(102-38 f / 3) / 16 \\
& \beta_{2}=\left(2857-5033 f / 9+325 f^{2} / 27\right) / 128
\end{aligned}
$$

which has the perturbative solution:

$$
\bar{a}\left(Q_{1}^{2}\right)=\bar{a}+\bar{a}^{2} \beta_{0} L_{1}+\bar{a}^{3}\left(\beta_{1} L_{1}+\beta_{0}^{2} L_{1}^{2}\right)+\bar{a}^{4}\left(\beta_{2} L_{1}+\frac{5}{2} \beta_{0} \beta_{1} L_{1}^{2}+\beta_{0}^{3} L_{1}^{3}\right),
$$

where $L_{1}=\log s / Q_{1}^{2}$ and $\bar{a}=\bar{a}(s)$. We also will need the analogous expansion of $a\left(Q_{2}^{2}\right)$ through 2 loops :

$$
\bar{a}\left(Q_{2}^{2}\right)=\bar{a}+\bar{a}^{2} \beta_{0} L_{2}+\bar{a}^{3}\left(\beta_{1} L_{2}+\beta_{0}^{2} L_{2}^{2}\right) .
$$

Next we substitute Eqs.(8.7,8.8) into Eq. (8.4), and expand, keeping terms through order $\bar{a}^{4}$. This yields the same $\bar{a}^{2}$ and $\bar{a}^{3}$ terms that we started with, and also gives the BLM prediction for the $a^{4}$ term :

$$
a_{H}(s)=\bar{a}+\bar{a}^{2}(A+B f)+\bar{a}^{3}\left(C+D f+E f^{2}\right)+\bar{a}^{4}\left(60-46 f+2.3 f^{2}-0.0081 f^{3}\right)
$$

To get an idea of how seriously to take these predictions, we have performed the analogous procedure on the one-loop conformal relation to predict the $\bar{a}^{3}$ terms. The 
result is

$$
a=\bar{a}+\bar{a}^{2}(A+B f)+\bar{a}^{3}\left(44-4.2 f+0.058 f^{2}\right) .
$$

Comparing with Eq.(8.3), we see that this gives roughly the correct answer for the $f$ and $f^{2}$ terms, with errors of $8 \%$ and $26 \%$, respectively. The $f^{0}$ term is not necessarily reliable, since it neglects the conformal contribution of that order. However, the analogous "prediction" in the $a^{3}$ case is 44 , whereas the correct result is 29 , indicating that perhaps the BLM "residue" (the $f^{0}$ term resulting from expanding the conformal relation) might be the same order of magnitude as the correct result which contains the conformal term as well. This is only the case if the conformal term is small compared to the non-conformal terms.

In any case we estimate that the $f, f^{2}$, and $f^{3} \times \bar{a}^{4}$ terms in Eq.(8.9) should be correct to about $30 \%$. The prediction for the total coefficient of the $\bar{a}^{4}$ term is -114 for the physically relevant case of $f=5$.

\subsubsection{Banks-Zaks Method}

A useful check of the BLM procedure is furnished by the Banks-Zaks method [126]. First, we solve for the value of $f$ that such that the three-loop $\beta$ function vanishes :

$$
f=\frac{33}{2}-\frac{321}{8} \bar{a}+\frac{11675}{256} \bar{a}^{2}
$$

At this number of flavors, the theory is effectively conformal. This value for $f$ is then substituted into Eq.(8.9), and it is verified that we recover the same conformal coefficients through order $a^{3}$ as predicted by the BLM method

$$
a=\bar{a}+\widetilde{A} \bar{a}^{2}+\widetilde{C} \bar{a}^{3} .
$$

Note there is no $\bar{a}^{4}$ conformal term until it is explicitly calculated. 


\subsubsection{Results and Limitations}

The full five-loop results reported in [124] are

$a_{H}(s)=\bar{a}+\bar{a}^{2}(A+B f)+\bar{a}^{3}\left(C+D f+E f^{2}\right)+\bar{a}^{4}\left(6.95-39.03 f+1.71 f^{2}-0.0036 f^{3}\right)$,

which is in reasonably good agreement with the predictions in Eq.(8.9). For $f=5$, the $\bar{a}^{4}$ term is -146 , to be compared with our prediction of -114 .

Finally, the conformal relation for the full NNNLO result is ${ }^{1}$

$$
a_{H}(s) \approx \bar{a}\left(Q_{1}^{2}\right)+2.39 \bar{a}^{2}\left(Q_{2}^{2}\right)-24.0 \bar{a}^{3}\left(Q_{3}^{2}\right)-76.3 \bar{a}^{4}\left(Q_{3}^{2}\right)
$$

while the usual fixed scale relation is

$$
a_{H}(s) \approx \bar{a}(s)+5.15 \bar{a}^{2}(s)+7.37 \bar{a}^{3}(s)-146 \bar{a}^{4}(s) .
$$

It is interesting that the latter result seems more convergent than the conformal relation until the NNNLO terms are added. The somewhat better overall behavior of the conformal relation at NNNLO might be interpreted as the absence of $n$ ! renormalon growth, but this is inconclusive, since the NNLO conformal coefficient is rather large compared with the NLO coefficient.

In the preceding analysis, it was assumed that all of the flavor dependence is associated with the QCD $\beta$ function. In that case one can use the $f$ dependence to identify the non-conformal contributions. However, at higher orders there are more complicated triangle and light-by-light diagrams which are not obviously associated with the running coupling. However, in Chapter 5 we demonstrate that the dominant part of triangle diagrams are related to coupling renormalization, albeit in a novel way. Similar considerations should hold for the box diagrams. Thus it is assumed that the $f$ dependence coming from fermion triangle and fermion box diagrams renormalize the three and four gluon vertices, respectively.

The ability to predict higher order non-conformal terms using BLM methods is

\footnotetext{
${ }^{1} Q_{3}$ is the NNLO BLM scale which has not been specified since the Banks-Zaks method was used to derive the conformal series. It is a tedious exercise to derive this scale.
} 
limited by: (1) ignorance of the BLM scale of the highest order term, (2) ignorance of the higher order corrections to the lower order BLM scales (for example the $\bar{a}^{2}$ terms in $Q_{1}$ in our case), and (3) terms proportional to $f$ from the light-by-light and fermion triangle diagrams which should be considered conformal.

\subsection{An Inquiry into the Dominance of Renormalons, BLM Methods, and Effective Charges Run- ning Inside of Loops}

At the end of section 2.1.3, we discussed how the dominance of renormalon-type contributions at each order in perturbation theory was a key ingredient for the conjectured divergence of perturbation theory. In general, this dominance conjecture is very difficult to test since it involves higher order calculations. However, the relation between the quark pole mass $M_{Q}$ and running $\overline{M S}$ mass $\bar{m}(\mu)$ provides a simple testing ground for this assumption of the renormalon analysis. In particular, we would like to see if the renormalon-type graphs (shown in Fig.(8.1)) are in fact the dominant contribution at the two-loop level. By "renormalon-type", we mean precisely the first iteration of the infinite sequence of gauge-invariant bubble-chain graphs shown in Fig.(2.1). To reliably re-sum these (and only these) N-bubble graphs, they should give the dominant contribution to the full $N+1$ loop calculation. The first order at which this can be tested is at two-loops $(N=1)$. The full two-loop graphs are shown in Fig.(8.2), and were calculated in [118]. The notation of [118] will be adopted here.

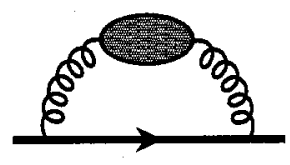

Figure 8.1: The renormalon-inspired approximation to the quark mass at two-loops. The blob represents some gauge-invariant gluon self-energy including gluons, ghosts, and quarks. 


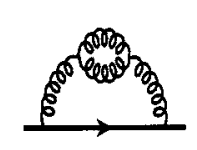

(a)

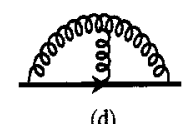

(d)

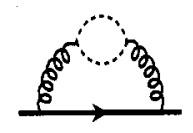

(b)

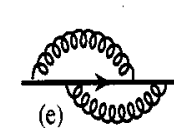

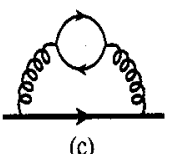

(c)

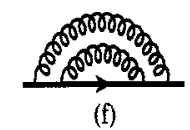

Figure 8.2: The two-loop graphs renormalizing the quark mass. The quark loops in (c) include both the light quarks and the massive quark under consideration.

To two-loop order, the mass relation can be written as

$$
M_{Q}=\bar{m}\left(M_{Q}\right)\left[1+C_{F} \bar{a}\left(M_{Q}\right)+D_{2} \bar{a}^{2}\left(M_{Q}\right)\right]
$$

where $\bar{a}\left(M_{Q}\right)=\bar{\alpha}\left(M_{Q}\right) / \pi$. The one-loop term is easily calculated to be $C_{F}=4 / 3$ while the two-loop term was found in Ref.[118], and can be written numerically as

$$
\begin{aligned}
& D_{2} \approx 3.33 C_{A} C_{F}+1.51 C_{F}^{2}+D_{2}(\text { fermion loops }) \\
& D_{2}(\text { fermion loops }) \approx 0.08 C_{F}-0.78 C_{F} \sum_{i=1}^{N_{F}-1}\left(1-M_{i} / M_{Q}\right),
\end{aligned}
$$

where the linear mass terms are an approximation to the exact result, and in any case are not important for out purposes. For the case of QCD $C_{F}=4 / 3$ and $C_{A}=3$ and this becomes

$$
D_{2} \approx 16.11-1.04 \sum_{i=1}^{N_{F}-1}\left(1-M_{i} / M_{Q}\right)
$$

\subsubsection{A Renormalon Inspired Approximation}

In order to obtain an analogous result in the renormalon-inspired approximation (RIA), only a few simple changes must be made to the results of Ref.[118]. In that paper, the two-loop term is written as a sum over color factors and loop factors, with coefficients $C_{i j}$ which depend only on the dimension of spacetime $d$. These coefficients, 
which are given in Table 1 of Ref.[118], will be changed in the RIA to

$$
\begin{aligned}
& C_{11}=C_{13}=C_{21}=C_{22}=C_{23}=0 \\
& C_{12}=-\frac{16}{3} \widetilde{\beta}_{0}(d) \frac{(d-1)}{(3 d-8)(3 d-10)},
\end{aligned}
$$

while $C_{31}, C_{32}, C_{33}, C_{41}, C_{42}$, and $C_{43}$ remain unchanged. In the same manner as Ref.[118], a linear combination of these coefficients with color factors and loop factors yield the results for the two-loop term $D_{2}$ given below for the RIA. Here $\widetilde{\beta}_{0}(d)$ is a function which parameterizes the non-abelian part of the gauge-invariant gluon self-energy insertion

$$
\widetilde{\Pi}\left(p^{2}\right)=i g^{2} C_{A} \widetilde{\beta}_{0}(d) \int \frac{d^{d} l}{(2 \pi)^{d}} \frac{1}{l^{2}(l+p)^{2}}
$$

as shown in Fig.(8.1). Only the purely non-abelian part is included in this definition since the fermion part is unchanged in the RIA (which is reflected in the fact that $C_{31}, C_{32}, C_{33}, C_{41}, C_{42}$, and $C_{43}$ remain unchanged). In four dimensions $\widetilde{\beta}_{0}(d)$ must take the specific value $\widetilde{\beta}_{0}(4)=11 / 3$. Different schemes for defining $\widetilde{\Pi}$ lead to different forms of this function, and subsequently different predictions for $D_{2}$ due to the finite terms from the expansion in dimensional regularization of $d=4-2 \epsilon$. This can be parameterized in general by

$$
\widetilde{\beta_{0}}(d)=\frac{11}{3}+a \epsilon+b \epsilon^{2}
$$

The results for the RIA are then

$$
\begin{aligned}
D_{2} & =(4.81+0.36 a+0.09 b) C_{A} C_{F}-3.89 C_{F}^{2}+D_{2} \text { (fermion loops) } \\
& =13.48+1.44 a+0.38 b-1.04 \sum_{i=1}^{N_{F}-1}\left(1-M_{i} / M_{Q}\right) .
\end{aligned}
$$

Before proceeding, it should be admitted that in fact no satisfactory method exists for constructing a gauge-invariant running charge inside of loops. However, we will proceed using various ansatz for this coupling, which amounts to specifying the form 
of $\widetilde{\beta_{0}}$ and thus $a$ and $b$.

First, let us consider the naive values $a=b=0$, for which the term 13.48 in Eq.(8.22) is in very reasonable agreement with the exact result 16.11 in Eq.(8.18). However, since the fermion bubble graphs are unchanged in the RIA, a better test is to see whether the individual color coefficients are in agreement.

The $C_{A} C_{F}$ term in the first line comes from Fig.(8.1) in the RIA, and from graphs (a), (b), and (d) of Fig.(8.2) in the full two-loop case. This term is only within $50 \%$ of the exact result. Meanwhile, the $C_{F}^{2}$ term in the exact result arises from graphs (e) and (f) in Fig.(8.2) and from the square of the one-loop graph. In the RIA, only the latter contribution is present and so the clear disagreement between the $C_{F}^{2}$ terms in Eqs. $(8.17,8.22)$ is expected. Thus the better agreement of Eq.(8.18) with Eq.(8.22) compared to the individual color factors is due to an accidental cancelation between the errors in $C_{A} C_{F}$ and $C_{F}^{2}$ terms for the particular value $N_{c}=3$.

Now let us turn to other possibilities. For example, the self-energy of the pinchtechnique (PT)/background field method in quantum Feynman gauge (BFMFG) has the value ${ }^{2}$

$$
\widetilde{\beta}_{0}(d)=\frac{7 d-6}{2(d-1)},
$$

as seen from Eqs. $(5.37,5.36)$, so that $a=1 / 9$ and $b=2 / 27$. This ansatz leads to

$$
\begin{aligned}
D_{2} & =4.86 C_{A} C_{F}-3.89 C_{F}^{2}+D_{2} \text { (fermion loops) } \\
& =13.67-1.04 \sum_{i=1}^{N_{F}-1}\left(1-M_{i} / M_{Q}\right)
\end{aligned}
$$

\footnotetext{
${ }^{2} \mathrm{~A}$ caveat is in order. The procedure of inserting the PT/BFMFG gauge-invariant self-energy into the loop is questionable in both the PT framework and in the equivalent BFMFG framework. In the BFMFG, it is the background gluons which have gauge-invariant self-energy, giving Eq.(8.23). However, these background gluons cannot propagate in loops; they may only appear in external legs. Moreover, the quantum gluons which propagate in loops do not have a gauge-invariant self-energy, but instead the usual gauge-dependent one that gives the coefficient $5 / 3$ rather than $11 / 3$. From the PT point of view, the difficulty can be seen by explicitly attempting the pinch-technique construction starting from the graphs in Fig.(8.2). It becomes clear that there is no natural kinematic criterion as in the on-shell case, where physical (external) gluon momenta can be singled out. The pinching procedure inside of loops is ambiguous and in general leads to new unwanted vertices. Nevertheless, we know of no other possible QCD effective charge which has been suggested to run inside of loops, so we persist in testing the RIA despite the above objections. After all, this is merely a theoretical Gedanken experiment.
} 
which is very similar to the naive $a=b=0$ case.

Now let us heuristically consider having the $\overline{M S}$ coupling running in the loop ${ }^{3}$. First, expanding around four dimensions, we find

$$
\widetilde{\Pi}\left(p^{2}\right)=-\frac{\alpha_{0}}{4 \pi} C_{A}\left(\frac{11}{3}\left(C_{U V}-\log \frac{-p^{2}}{\mu^{2}}\right)+a+\frac{22}{3}\right)
$$

where $\alpha_{0}=g_{0}^{2} /(4 \pi)$ is the bare coupling and $C_{U V}=\frac{1}{\epsilon}-\gamma_{E}+\log 4 \pi$. The value of the parameter ' $a$ ' can be fixed by the requirement that re-summing this self-energy leads to the $\overline{M S}$ coupling. Since we know that the $\overline{M S}$ coupling is related to the bare coupling by

$$
\bar{\alpha}\left(Q^{2}\right) \approx \alpha_{0}\left[1-\frac{\alpha_{0}}{4 \pi} \frac{11}{3} C_{A}\left(\log \frac{Q^{2}}{\mu^{2}}-C_{U V}\right)\right]
$$

it must be that $a=-22 / 3$. This argument does not fix $b$. However, if the well motivated parametrization $\widetilde{\beta}_{0}(d)=\frac{11+c(d-4)}{d-1}$ is assumed, then $b=2 a / 3$. In any case, the results are fairly insensitive to $b$. We have

$$
\begin{aligned}
D_{2} & =1.72 C_{A} C_{F}-3.89 C_{F}^{2}+D_{2}(\text { fermion loops }) \\
& =1.10-1.04 \sum_{i=1}^{N_{F}-1}\left(1-M_{i} / M_{Q}\right)
\end{aligned}
$$

which is in terrible agreement with the correct results, Eqs. $(8.17,8.18)$.

Similar machinations using the V-scheme effective charge [103] defined from the heavy quark potential also lead to results in very poor agreement.

\subsubsection{BLM Methods}

Now let us turn to a BLM analysis similar to that of section 8.1. An effective charge $a_{Q}=\alpha_{Q} / \pi$ associated with the $\overline{M S} /$ pole mass relation is defined from the original

\footnotetext{
${ }^{3}$ Again, we know of no construction that could accomplish this. See the previous footnote.
} 
series in Eq.(8.17) to absorb all of the radiative corrections (see Eq.(3.4))

$$
\begin{aligned}
M_{Q} & \equiv \bar{m}\left(M_{Q}\right)\left[1+C_{F} a_{Q}\left(M_{Q}\right)\right] \\
a_{Q}\left(M_{Q}\right) & \approx \bar{a}\left(M_{Q}\right)+\bar{a}^{2}\left(M_{Q}\right)\left[3.33 C_{A}+1.51 C_{F}+0.08-0.78\left(N_{F}-1\right)\right] \\
& \approx \bar{a}\left(M_{Q}\right)+\bar{a}^{2}\left(M_{Q}\right)\left[12.78-0.78 N_{F}\right],
\end{aligned}
$$

where $\bar{a}=\bar{\alpha} / \pi$ is the $\overline{M S}$ coupling and for simplicity we have neglected the lightquark mass corrections. For the charm, bottom, and top quarks we have

$$
a_{Q=(c, b, t)}\left(M_{Q}\right) \approx \bar{a}\left(M_{Q}\right)+(9.74,8.96,8.18) \bar{a}^{2}\left(M_{Q}\right)
$$

Meanwhile, the conformal relation for $a_{Q}$ obtained using BLM methods is

$$
a_{Q}\left(M_{Q}\right) \approx \bar{a}\left(Q_{1}\right)-0.80 \bar{a}^{2}\left(Q_{1}\right)
$$

where $Q_{1} \approx 0.096 M_{Q}$. The conformal coefficient is quite small, suggesting that the dominant source of radiative corrections are non-conformal, i.e. associated with the running coupling.

We can also use Eq. (8.30) to predict the NNLO (three-loop) correction term, using the same methods as section 8.1. The BLM prediction for the $\bar{a}^{3}$ term is

$$
a_{Q}\left(M_{Q}\right) \approx \bar{a}\left(M_{Q}\right)+\bar{a}^{2}\left(M_{Q}\right)\left[12.78-0.78 N_{F}\right]+\bar{a}^{3}\left(M_{Q}\right)\left[175.2-22.6 N_{F}+0.61 N_{F}^{2}\right],
$$

which can be compared to the recently completed exact three-loop calculation [119]

$$
a_{Q}\left(M_{Q}\right) \approx \bar{a}\left(M_{Q}\right)+\bar{a}^{2}\left(M_{Q}\right)\left[12.78-0.78 N_{F}\right]+\bar{a}^{3}\left(M_{Q}\right)\left[179.5-22.3 N_{F}+0.49 N_{F}^{2}\right] .
$$

Thus, the BLM prediction is in remarkable agreement with the exact results, with errors of $2 \%, 1 \%$, and $24 \%$ for the $N_{F}^{0} \bar{a}^{3}, N_{F}^{1} \bar{a}^{3}$, and $N_{F}^{2} \bar{a}^{3}$ terms, respectively. Furthermore, the prediction for the full $\bar{a}^{3}$ term is accurate to within $3-4 \%$ for the physically relevant cases of charm, bottom, and top quarks.

Since the conformal coefficient is small and the BLM predictions are so accurate, in 
the BLM parlance one would associate most of the radiative corrections at this order of perturbation theory with the running of the coupling. However, our above attempt at an explicit construction of these gauge-invariant contributions in the renormaloninspired-approximation generally did not reproduce the correct results and were in fact plagued by scheme ambiguities. These results suggest that perhaps the success of the BLM method may not be tied to a hypothetical skeleton expansion with running charges inside of loops.

Furthermore, it seems that the dominance assumption of the renormalon analysis is questionable in this case, although it certainly may be the case that renormalontype graphs only begin to dominate at higher orders. The analysis is plagued by ambiguities in how to actually construct the gauge-invariant running charge inside of loops. These interesting issues deserve further study. 


\section{Chapter 9}

\section{Future Directions}

The results of this thesis can be extended in a number of directions:

Four-gluon vertex A calculation similar to the one presented in Chapter 5 would be interesting. Of particular interest is whether the logarithm-like function of the four-gluon vertex, $L_{4}\left(k_{1}, k_{2}, k_{3}, k_{4}\right)$, analogous to Eq.(5.73), has the same form for gluons, quarks, and scalars in the massless limit. Also, the general behavior of the effective scale of the four-gluon vertex will be useful for phenomenology. However, the calculation is challenging since in this case there are hundreds of distinct form factors.

Standard Model triple-gauge-boson vertices and unification The form factors of the gauge-invariant triple gauge boson vertices of the Standard Model can be calculated in analogy with Chapter 5 . It might be interesting to look at the multiscale unification of the triple gauge boson vertices. In this way, one would see the unification of the strength of the forces between (three) test fermions, in analogy with Chapter 3. To be clear, this is not an independent test of unification (even if the various form factors were measured very precisely), but rather it gives a nice qualitative picture of how the various forces reconstruct back into one force smoothly at the GUT scale. 
Monte Carlo Generator A program which constructs any process by stringing together trees of primal gauge-invariant PT/BFMFG Green's functions, which can be renormalized independently. The approach presented in Chapter 5 can be applied to other Green's functions, as discussed above, and represents an optimal improvement of perturbation theory. Thus, for example, a process fully dressed at tree-level is expected to be a good approximation of the full one-loop result. Since the gaugeinvariant Green's functions are universal, only a small set of formula is required input into such a program.

Heavy quark hadroproduction As an application of the results of chapter 5, a preliminary analysis has been made of heavy quark production in proton-proton collisions. In particular, we can study the three-gluon vertex by considering the production of two heavy quarks plus two associated transverse jets in proton-proton collisions :

$$
p p \Rightarrow Q \bar{Q}+2 \text { jets }\left(p_{T} \neq 0\right)
$$

which is illustrated in Fig.(9.1). The various kinematic variables control the gluon virtualities, and so in principle one can see the non-intuitive behavior and running the three-gluon vertex discussed in section 5.4. Preliminary results suggest that the effective scale is much lower than would be expected, and so the overall process is enhanced compared to the $\overline{M S}$ scheme predictions when the scale is chosen as the heavy quark pair invariant mass. It is believed that the tree level graphs dressed with gauge-invariant self-energies (Chapter 6) and vertices (Chapter 5) account for most of the full one-loop corrections. However, this can not be tested until the full one-loop calculation of the $2 \rightarrow 4$ process is performed.

Survey and analysis of perturbation theory Inspired by the preliminary results of Chapter 8, it would be useful to undertake a comprehensive study of perturbative QCD series, taking into account as many observables as possible. For each, BLM, FAC, and PMS (see section 2.1.1) can be used at each order to "predict" the next higher order terms, and then compared with the actual results. This should yield a large sample of "data" to compare the scale-fixing approaches rigorously. Also it 

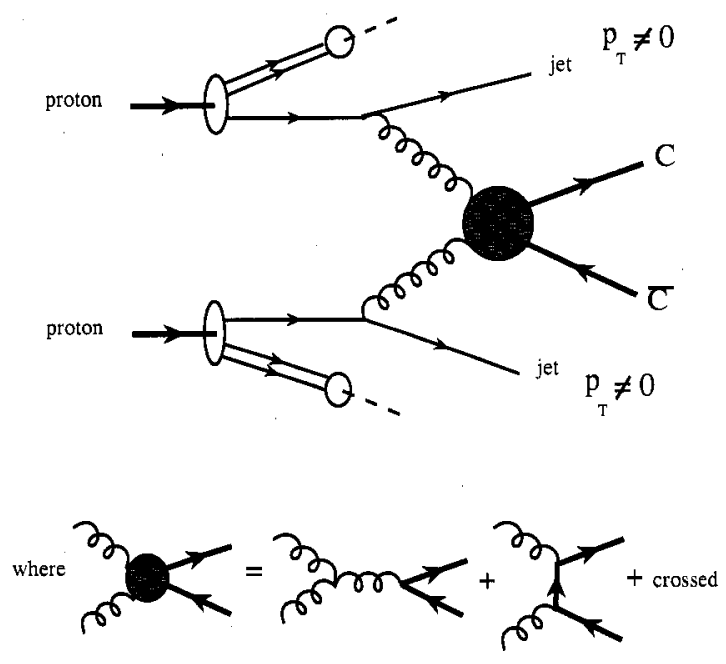

Figure 9.1: The hadro-production of heavy quarks and two associated transverse jets, as a direct probe of the three-gluon vertex.

would be interesting to see which observables seem to have the $n$ ! (renormalon) growth in their series, and which observables have relatively small conformal coefficients. What, if any, relation is there between successful predictions, renormalon growth of the series, and conformal coefficients? For some observables the "renormaloninspired-approximation" of section 8.2 could be computed as well, and used to further understand these issues.

Dyson-Schwinger Equations A new approach to Dyson-Schwinger equations (DSE's) is suggested by

(1) the equivalence of the pinch-technique and the background field method in quantum Feynman gauge, and

(2) the arguments given in the footnote below Eq.(8.23) of section 8.2.1 that the gauge-invariant Green's functions should not appear in loops.

There seems to be a "two-level" structure, which is best understood in the BFMFG 
approach. First one solves the DSE's for the quantum Green's functions, which satisfy the normal DSE's, but in Feynman gauge. Then the resulting solutions are "processed" through a one-loop integral representing a graph with external background gluons attached. This is illustrated in Fig.(9.2) for the gluon propagator.

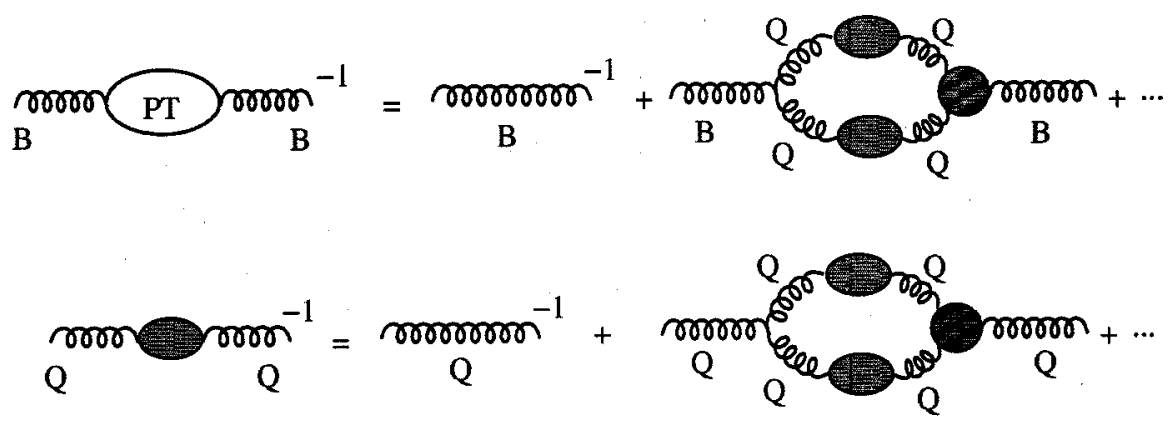

Figure 9.2: A conjecture on the structure of DSE's in the PT/BFMFG approach. The quantum (Q) gluon Green's functions satisfy the typical recursive DSE's shown in the bottom line, which should be solved in the Feynman gauge. These solutions are then "processed" through a (non-recursive) one-loop type of integral, shown in the top line, to form the gauge-invariant PT/BFMFG Green's function of the background (B) gluons (the blob labeled 'PT'). Other terms with four-gluon vertices, fermions, and ghosts are not shown explicitly.

One of the original motivations for the PT [30] was to develop manifestly gaugeinvariant approach to DSE's in order to study confinement and dynamical gluon mass generation. The conjectured approach presented here may provide a solution to this problem, albeit circuitously. The gauge-invariant PT/BFMFG Green's functions of the background (B) gluons themselves do not appear recursively, but they arise out the solution of the DSE's for the (un-physical) quantum (Q) gluons, which must be solved in Feynman gauge. In some sense there is no gauge-arbitrariness and it is plausible that a reasonable truncation of the quantum gluon DSE's will still result in a gauge-invariant PT self-energy. This issue deserves more study. In contrast to 
this conjectured approach, perhaps a new method will be found to incorporate the gauge-invariant Green's functions inside of loops and have them directly satisfy a manifestly gauge-invariant set of DSE's, as originally envisaged in Ref.([30]). 


\section{Bibliography}

[1] M. Binger and S. J. Brodsky, Phys. Rev. D 69, 095007 (2004) [arXiv:hep$\mathrm{ph} / 0310322]$.

[2] M. Binger and S. J. Brodsky, arXiv:hep-ph/0602199.

[3] M. Binger, Phys. Rev. D 73, 095001 (2006) [arXiv:hep-ph/0408240].

[4] S. L. Glashow, "Partial Symmetries Of Weak Interactions," Nucl. Phys. 22, 579 (1961). S. Weinberg, "A Model Of Leptons," Phys. Rev. Lett. 19, 1264 (1967). A. Salam, in Elementary Particle Theory, N. Svartholm, ed. (Stockholm: Almquist and Wiksell, 1968).

[5] H. E. Haber and G. L. Kane, Phys. Rept. 117, 75 (1985).

[6] C. T. Hill and E. H. Simmons, Phys. Rept. 381, 235 (2003) [Erratum-ibid. 390, 553 (2004)] [arXiv:hep-ph/0203079].

[7] I. Antoniadis, N. Arkani-Hamed, S. Dimopoulos and G. R. Dvali, Phys. Lett. B 436, 257 (1998) [arXiv:hep-ph/9804398].

[8] M. Schmaltz and D. Tucker-Smith, arXiv:hep-ph/0502182.

[9] P. M. Stevenson, Phys. Rev. D 23, 2916 (1981). Nucl. Phys. B 203, 472 (1982).

[10] G. Grunberg, Phys. Lett. B 95, 70 (1980) [Erratum-ibid. B 110, 501 (1982)].

[11] G. Grunberg, Phys. Rev. D 29, 2315 (1984).

[12] S. J. Brodsky, G. P. Lepage and P. B. Mackenzie, Phys. Rev. D 28, 228 (1983). 
[13] D. V. Shirkov and I. L. Solovtsov, arXiv:hep-ph/9604363.

[14] S. J. Brodsky, S. Menke, C. Merino and J. Rathsman, Phys. Rev. D 67, 055008 (2003) [arXiv:hep-ph/0212078].

[15] R. Alkofer, C. S. Fischer and F. J. Llanes-Estrada, Phys. Lett. B 611, 279 (2005) [arXiv:hep-th/0412330].

[16] M. Neubert, Phys. Rev. D 51, 5924 (1995) [arXiv:hep-ph/9412265].

[17] M. Neubert, arXiv:hep-ph/9502264.

[18] S. J. Brodsky and P. Huet, Phys. Lett. B 417, 145 (1998) [arXiv:hep$\mathrm{ph} / 9707543]$.

[19] D. A. Ross, Nucl. Phys. B 140, 1 (1978).

[20] D. C. Kennedy and B. W. Lynn, Nucl. Phys. B 322, 1 (1989). SLAC-PUB-4608 B. W. Lynn, SLAC-PUB-5077

[21] M. Kreuzer and W. Kummer, Phys. Lett. B 197, 389 (1987) [Erratum-ibid. 208B, 530 (1988)]. M. Kreuzer, W. Kummer and A. Rebhan, Z. Phys. C 44, 479 (1989).

[22] A. E. Faraggi and B. Grinstein, Nucl. Phys. B 422, 3 (1994) [arXiv:hep$\mathrm{ph} / 9308329]$.

[23] J. Bagger, K. T. Matchev and D. Pierce, Phys. Lett. B 348, 443 (1995) [arXiv:hep-ph/9501277].

[24] L. Clavelli and P. W. Coulter, arXiv:hep-ph/9507261.

[25] M. Bastero-Gil and J. Perez-Mercader, Nucl. Phys. B 450, 21 (1995) [arXiv:hep$\mathrm{ph} / 9506222]$.

[26] M. Bastero-Gil, arXiv:hep-ph/9610340. 
[27] S. J. Brodsky, M. S. Gill, M. Melles and J. Rathsman, Phys. Rev. D 58, 116006 (1998) [arXiv:hep-ph/9801330].

[28] S. D. Bass, R. J. Crewther, F. M. Steffens and A. W. Thomas, Phys. Rev. D 68, 096005 (2003) [arXiv:hep-ph/0211376]. Phys. Rev. D 66, 031901 (2002) [arXiv:hep-ph/0207071].

[29] For an early attempt at constructing electroweak effective charges see L. Baulieu and R. Coquereaux. Phys. Lett. B96, 345 (1980).

[30] J. M. Cornwall, Phys. Rev. D 26, 1453 (1982).

[31] G. Degrassi and A. Sirlin, Phys. Rev. D 46, 3104 (1992).

[32] N. J. Watson, Nucl. Phys. B 494, 388 (1997) [arXiv:hep-ph/9606381].

[33] J. Papavassiliou, E. de Rafael and N. J. Watson, Nucl. Phys. B 503, 79 (1997) [arXiv:hep-ph/9612237].

[34] J. Papavassiliou and A. Pilaftsis, Phys. Rev. D 54, 5315 (1996) [arXiv:hep$\mathrm{ph} / 9605385]$.

[35] J. Papavassiliou, Phys. Rev. Lett. 84, 2782 (2000) [arXiv:hep-ph/9912336].

[36] J. Papavassiliou, Phys. Rev. D 62, 045006 (2000) [arXiv:hep-ph/9912338].

[37] D. Binosi and J. Papavassiliou, Phys. Rev. D 66, 111901 (2002) [arXiv:hep$\mathrm{ph} / 0208189]$.

[38] D. Binosi and J. Papavassiliou, Nucl. Phys. Proc. Suppl. 121, 281 (2003) [arXiv:hep-ph/0209016].

[39] D. Binosi, J. Phys. G 30, 1021 (2004) [arXiv:hep-ph/0401182].

[40] J. Schwinger. Particles, Sources, and Fields, Vol. 1 (1970). 
[41] One way to see this is by looking at the mass dependent analytic 2 loop results for the related heavy quark effective charge : M. Melles. Phys. Rev. D 62, 074019 (2000). hep-ph/0001295. S.J. Brodksy, M. Melles, and J. Rathsman. Phys. Rev. D 60, 096006 (1999). hep-ph/9906324.

[42] N. Polonsky, UMI-95-21103

[43] K. R. Dienes, Phys. Rept. 287, 447 (1997) [arXiv:hep-th/9602045].

[44] V. S. Kaplunovsky, arXiv:hep-th/9205070.

[45] T. Friedmann and E. Witten, Adv. Theor. Math. Phys. 7, 577 (2003) [arXiv:hepth/0211269].

[46] M. E. Machacek and M. T. Vaughn, Nucl. Phys. B 222, 83 (1983). Nucl. Phys. B 236, 221 (1984). Nucl. Phys. B 249, 70 (1985).

[47] M. x. Luo and Y. Xiao, Phys. Rev. Lett. 90, 011601 (2003) [arXiv:hepph/0207271]. M. x. Luo, H. w. Wang and Y. Xiao, Phys. Rev. D 67, 065019 (2003) [arXiv:hep-ph/0211440].

[48] D. R. T. Jones, Phys. Rev. D 25, 581 (1982).

[49] R. Slansky, Phys. Rept. 79, 1 (1981).

[50] R. Hempfling, Phys. Lett. B 351, 206 (1995) [arXiv:hep-ph/9502201].

[51] D. M. Pierce, J. A. Bagger, K. T. Matchev and R. j. Zhang, Nucl. Phys. B 491, 3 (1997) [arXiv:hep-ph/9606211].

[52] Q. Shafi and C. Wetterich, Phys. Rev. Lett. 52, 875 (1984).

[53] C. T. Hill, Phys. Lett. B 135, 47 (1984).

[54] L. J. Hall and U. Sarid, Phys. Rev. Lett. 70, 2673 (1993) [arXiv:hep-ph/9210240].

[55] K. Tobe and J. D. Wells, Phys. Lett. B 588, 99 (2004) [arXiv:hep-ph/0312159]. 
[56] M. Battaglia, A. De Roeck, J. R. Ellis, F. Gianotti, K. A. Olive and L. Pape, Eur. Phys. J. C 33, 273 (2004) [arXiv:hep-ph/0306219].

[57] N. Arkani-Hamed and S. Dimopoulos, JHEP 0506, 073 (2005) [arXiv:hepth/0405159].

[58] G. F. Giudice and A. Romanino, Nucl. Phys. B 699, 65 (2004) [Erratum-ibid. B 706, 65 (2005)] [arXiv:hep-ph/0406088].

[59] K. S. Babu, J. C. Pati and F. Wilczek, Nucl. Phys. B 566, 33 (2000) [arXiv:hep$\mathrm{ph} / 9812538]$.

[60] J. M. Cornwall and J. Papavassiliou, Phys. Rev. D 40, 3474 (1989).

[61] R. Alkofer and L. von Smekal, Phys. Rept. 353, 281 (2001) [arXiv:hep$\mathrm{ph} / 0007355]$.

[62] S. J. Brodsky, E. Gardi, G. Grunberg and J. Rathsman, Phys. Rev. D 63, 094017 (2001) [arXiv:hep-ph/0002065].

[63] M. Beneke, Phys. Rept. 317, 1 (1999) [arXiv:hep-ph/9807443].

[64] K. Philippides and A. Sirlin, Phys. Lett. B 367, 377 (1996) [arXiv:hep$\mathrm{ph} / 9510393]$.

[65] J. Papavassiliou and A. Sirlin, Phys. Rev. D 50, 5951 (1994) [arXiv:hep$\mathrm{ph} / 9403378]$.

[66] J. Papavassiliou and K. Philippides, Phys. Rev: D 52, 2355 (1995) [arXiv:hep$\mathrm{ph} / 9503377]$.

[67] J. Papavassiliou, Phys. Rev. D 41, 3179 (1990).

[68] J. Papavassiliou, Phys. Rev. D 50, 5958 (1994) [arXiv:hep-ph/9406258].

[69] J. Papavassiliou, Phys. Rev. D 47, 4728 (1993). 
[70] J. Bernabeu, J. Papavassiliou and J. Vidal, Phys. Rev. Lett. 89, 101802 (2002) [Erratum-ibid. 89, 229902 (2002)] [arXiv:hep-ph/0206015].

[71] J. Papavassiliou and K. Philippides, Phys. Rev. D 48, 4255 (1993) [arXiv:hep$\mathrm{ph} / 9310210]$.

[72] Z. Bern, L. J. Dixon, D. C. Dunbar and D. A. Kosower, Nucl. Phys. B 425, 217 (1994) [arXiv:hep-ph/9403226].

[73] Z. Bern, L. J. Dixon, D. C. Dunbar and D. A. Kosower, Nucl. Phys. B 435, 59 (1995) [arXiv:hep-ph/9409265].

[74] Z. Bern and A. G. Morgan, Phys. Rev. D 49, 6155 (1994) [arXiv:hep$\mathrm{ph} / 9312218]$.

[75] D. Binosi and J. Papavassiliou, Phys. Rev. D 65, 085003 (2002) [arXiv:hep$\mathrm{ph} / 0110238]$.

[76] D. Binosi and J. Papavassiliou, J. Phys. G 30, 203 (2004) [arXiv:hep$\mathrm{ph} / 0301096]$.

[77] A. Denner, G. Weiglein and S. Dittmaier, Phys. Lett. B 333, 420 (1994) [arXiv:hep-ph/9406204].

[78] J. Papavassiliou, Phys. Rev. D 51, 856 (1995) [arXiv:hep-ph/9410385].

[79] L. F. Abbott, Nucl. Phys. B 185, 189 (1981). L. F. Abbott, M. T. Grisaru and R. K. Schaefer, Nucl. Phys. B 229, 372 (1983). L. F. Abbott, Acta Phys. Polon. B 13, 33 (1982).

[80] J. S. Ball and T. W. Chiu, Phys. Rev. D 22, 2542 (1980). Phys. Rev. D 22, 2550 (1980) [Erratum-ibid. D 23, 3085 (1981)].

[81] A. I. Davydychev, P. Osland and O. V. Tarasov, Phys. Rev. D 54, 4087 (1996) [Erratum-ibid. D 59, 109901 (1999)] [arXiv:hep-ph/9605348]. 
[82] Z. Bern, L. J. Dixon and D. A. Kosower, Ann. Rev. Nucl. Part. Sci. 46, 109 (1996) [arXiv:hep-ph/9602280].

[83] H. J. Lu, SLAC-PUB-5820 H. J. Lu and C. A. Perez, SLAC-PUB-5809 H. J. Lu, SLAC-0406

[84] A. I. Davydychev, P. Osland and L. Saks, JHEP 0108, 050 (2001) [arXiv:hep$\mathrm{ph} / 0105072]$.

[85] S. J. Brodsky, M. Melles and J. Rathsman, Phys. Rev. D 60, 096006 (1999) [arXiv:hep-ph/9906324].

[86] A. I. Davydychev and P. Osland, Phys. Rev. D 59, 014006 (1999) [arXiv:hep$\mathrm{ph} / 9806522]$.

[87] A. I. Davydychev, P. Osland and O. V. Tarasov, Phys. Rev. D 58, 036007 (1998) [arXiv:hep-ph/9801380].

[88] J. Fleischer, F. Jegerlehner, O. V. Tarasov and O. L. Veretin, Nucl. Phys. B 539, 671 (1999) [Erratum-ibid. B 571, 511 (2000)] [arXiv:hep-ph/9803493].

[89] G. 't Hooft and M. J. G. Veltman, Nucl. Phys. B 153, 365 (1979).

[90] G. J. van Oldenborgh and J. A. M. Vermaseren, Z. Phys. C 46, 425 (1990).

[91] A. I. Davydychev and R. Delbourgo, J. Math. Phys. 39, 4299 (1998) [arXiv:hepth/9709216].

[92] L. D. Landau, Nucl. Phys. 13, 181 (1959).

[93] R. Blankenbecler and Y. Nambu, Nuovo Cim. 18, 580 (1960).

[94] L. Lewin, "Polylogarithms and Associated Functions," (North-Holland, Amsterdam, 1981).

[95] A. Denner, G. Weiglein and S. Dittmaier, Nucl. Phys. B 440, 95 (1995) [arXiv:hep-ph/9410338]. 
[96] A. Denner, G. Weiglein and S. Dittmaier, Phys. Lett. B 333, 420 (1994) [arXiv:hep-ph/9406204]. J. Papavassiliou, Phys. Rev. D 51, 856 (1995) [arXiv:hep-ph/9410385].

[97] M. Binger and S. J. Brodsky, arXiv:hep-ph/0602199.

[98] D. M. Capper and A. MacLean, Nucl. Phys. B 203, 413 (1982).

[99] Z. Bern, A. De Freitas, L. J. Dixon and H. L. Wong, Phys. Rev. D 66, 085002 (2002) [arXiv:hep-ph/0202271].

[100] O. V. Tarasov, A. A. Vladimirov and A. Y. Zharkov, Phys. Lett. B 93, 429 (1980).

[101] S. A. Larin and J. A. M. Vermaseren, Phys. Lett. B 303, 334 (1993) [arXiv:hepph/9302208].

[102] T. Appelquist, M. Dine and I. J. Muzinich, Phys. Lett. B 69, 231 (1977).

[103] Y. Schroder, Phys. Lett. B 447, 321 (1999) [arXiv:hep-ph/9812205].

[104] R. Mahbubani, arXiv:hep-ph/0408096.

[105] B. Mukhopadhyaya and S. SenGupta, Phys. Rev. D 71, 035004 (2005) [arXiv:hep-th/0407225].

[106] A. Pierce, Phys. Rev. D 70, 075006 (2004) [arXiv:hep-ph/0406144].

[107] A. Arvanitaki, C. Davis, P. W. Graham and J. G. Wacker, Phys. Rev. D 70, 117703 (2004) [arXiv:hep-ph/0406034].

[108] W. Kilian, T. Plehn, P. Richardson and E. Schmidt, Eur. Phys. J. C 39, 229 (2005) [arXiv:hep-ph/0408088].

[109] J. L. Hewett, B. Lillie, M. Masip and T. G. Rizzo, JHEP 0409, 070 (2004) [arXiv:hep-ph/0408248]. 
[110] N. Arkani-Hamed, S. Dimopoulos, G. F. Giudice and A. Romanino, Nucl. Phys. B 709, 3 (2005) [arXiv:hep-ph/0409232].

[111] L. Susskind, arXiv:hep-th/0405189.

[112] R. Hempfling and A. H. Hoang, Phys. Lett. B 331, 99 (1994) [arXiv:hepph/9401219]. H. E. Haber, R. Hempfling and A. H. Hoang, Z. Phys. C 75, 539 (1997) [arXiv:hep-ph/9609331]. M. Carena, M. Quiros and C. E. M. Wagner, Nucl. Phys. B 461, 407 (1996) [arXiv:hep-ph/9508343].

[113] A. Sirlin and R. Zucchini, Nucl. Phys. B 266, 389 (1986).

[114] R. Hempfling and B. A. Kniehl, Phys. Rev. D 51, 1386 (1995) [arXiv:hep$\mathrm{ph} / 9408313]$.

[115] A. Sirlin, Phys. Rev. D 22, 971 (1980).

[116] S. Eidelman et al. [Particle Data Group], Phys. Lett. B 592, 1 (2004).

[117] [CDF Collaboration], arXiv:hep-ex/0507091.

[118] N. Gray, D. J. Broadhurst, W. Grafe and K. Schilcher, Z. Phys. C 48, 673 (1990).

[119] K. G. Chetyrkin and M. Steinhauser, Phys. Rev. Lett. 83, 4001 (1999) [arXiv:hep-ph/9907509]. Nucl. Phys. B 573, 617 (2000) [arXiv:hep-ph/9911434].

[120] H. Arason, D. J. Castano, B. Keszthelyi, S. Mikaelian, E. J. Piard, P. Ramond and B. D. Wright, Phys. Rev. D 46, 3945 (1992).

[121] M. Bohm, H. Spiesberger and W. Hollik, Fortsch. Phys. 34, 687 (1986).

[122] R. Barate et al. [LEP Working Group for Higgs boson searches], Phys. Lett. B 565, 61 (2003) [arXiv:hep-ex/0306033].

[123] K. G. Chetyrkin, Phys. Lett. B 390, 309 (1997) [arXiv:hep-ph/9608318]. 
[124] P. A. Baikov, K. G. Chetyrkin and J. H. Kuhn, Phys. Rev. Lett. 96, 012003 (2006) [arXiv:hep-ph/0511063].

[125] S. J. Brodsky and H. J. Lu, Phys. Rev. D 51, 3652 (1995) [arXiv:hep$\mathrm{ph} / 9405218]$.

[126] T. Banks and A. Zaks, Nucl. Phys. B 196, 189 (1982). 